Prepared in cooperation with Oregon Department of Environmental Quality and Deschutes County Environmental Health Division

Organic Wastewater Compounds, Pharmaceuticals, and Coliphage in Ground Water Receiving Discharge from Onsite Wastewater Treatment Systems near La Pine, Oregon: Occurrence and Implications for Transport

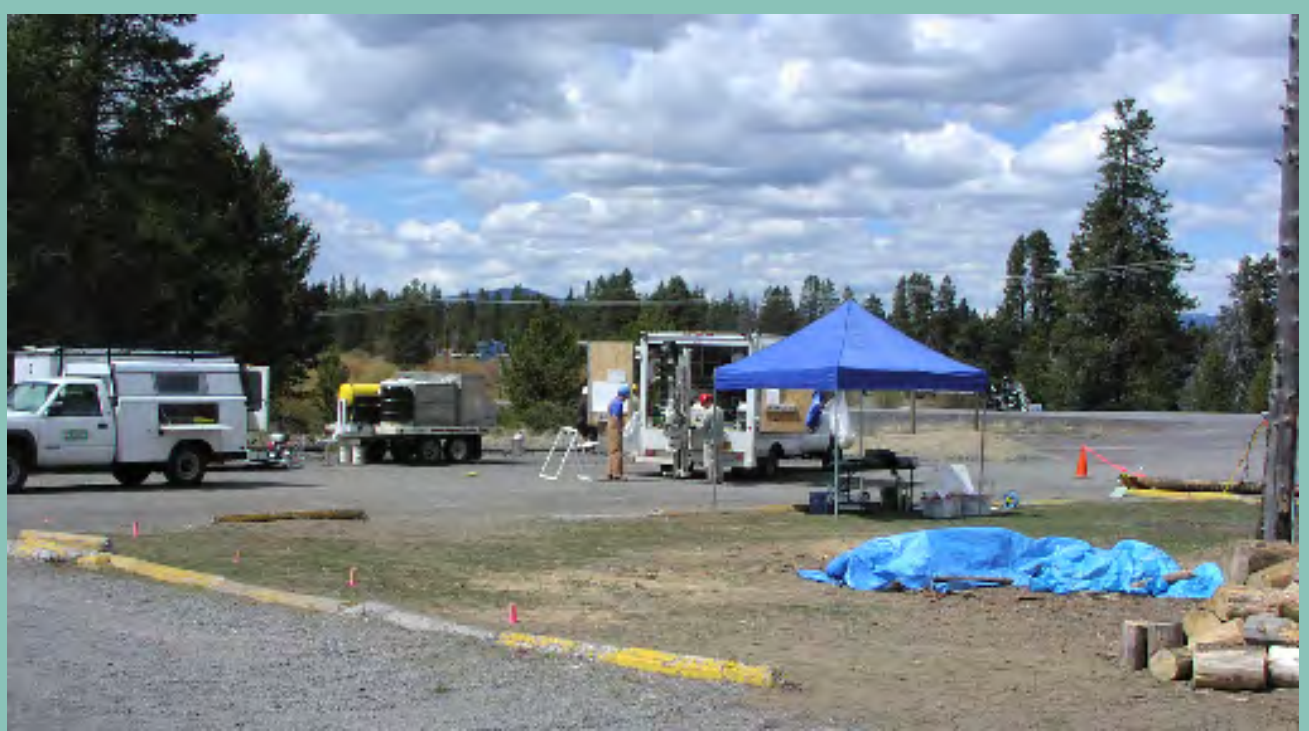

Scientific Investigations Report 2005-5055

Version 1.1, July 2009 
Front cover: A temporary, direct-push monitoring well is installed into a plume of septic tank effluent near La Pine, Oregon. 


\section{Organic Wastewater Compounds, Pharmaceuticals, and Coliphage in Ground Water Receiving Discharge from Onsite Wastewater Treatment Systems near La Pine, Oregon: Occurrence and Implications for Transport}

By Stephen R. Hinkle, Rodney J. Weick (Oregon Department of Environmental Quality), Jill M. Johnson (Deschutes County Environmental Health Division), Jeffery D. Cahill, Steven G. Smith, and Barbara J. Rich (Oregon Department of Environmental Quality)

Prepared in cooperation with Oregon Department of Environmental Quality and Deschutes County Environmental Health Division

Scientific Investigations Report 2005-5055

Version 1.1, July 2009 


\section{U.S. Department of the Interior \\ Gale A. Norton, Secretary \\ U.S. Geological Survey \\ Charles G. Groat, Director}

U.S. Geological Survey, Reston, Virginia: 2005

For more information about the USGS and its products:

Telephone: 1-888-ASK-USGS

World Wide Web: http://www.usgs.gov/

Any use of trade, product, or firm names in this publication is for descriptive purposes only and does not imply endorsement by the U.S. Government.

Suggested citation:

S.R. Hinkle, R.J. Weick, J.M. Johnson, J.D. Cahill, S.G. Smith, and B.J. Rich, 2005, Organic wastewater compounds, pharmaceuticals, and coliphage in ground water receiving discharge from onsite wastewater treatment systems near La Pine, Oregon-Occurrence and implications for transport: U.S. Geological Survey Scientific Investigations Report 2005-5055, $98 \mathrm{p}$. 


\section{Contents}

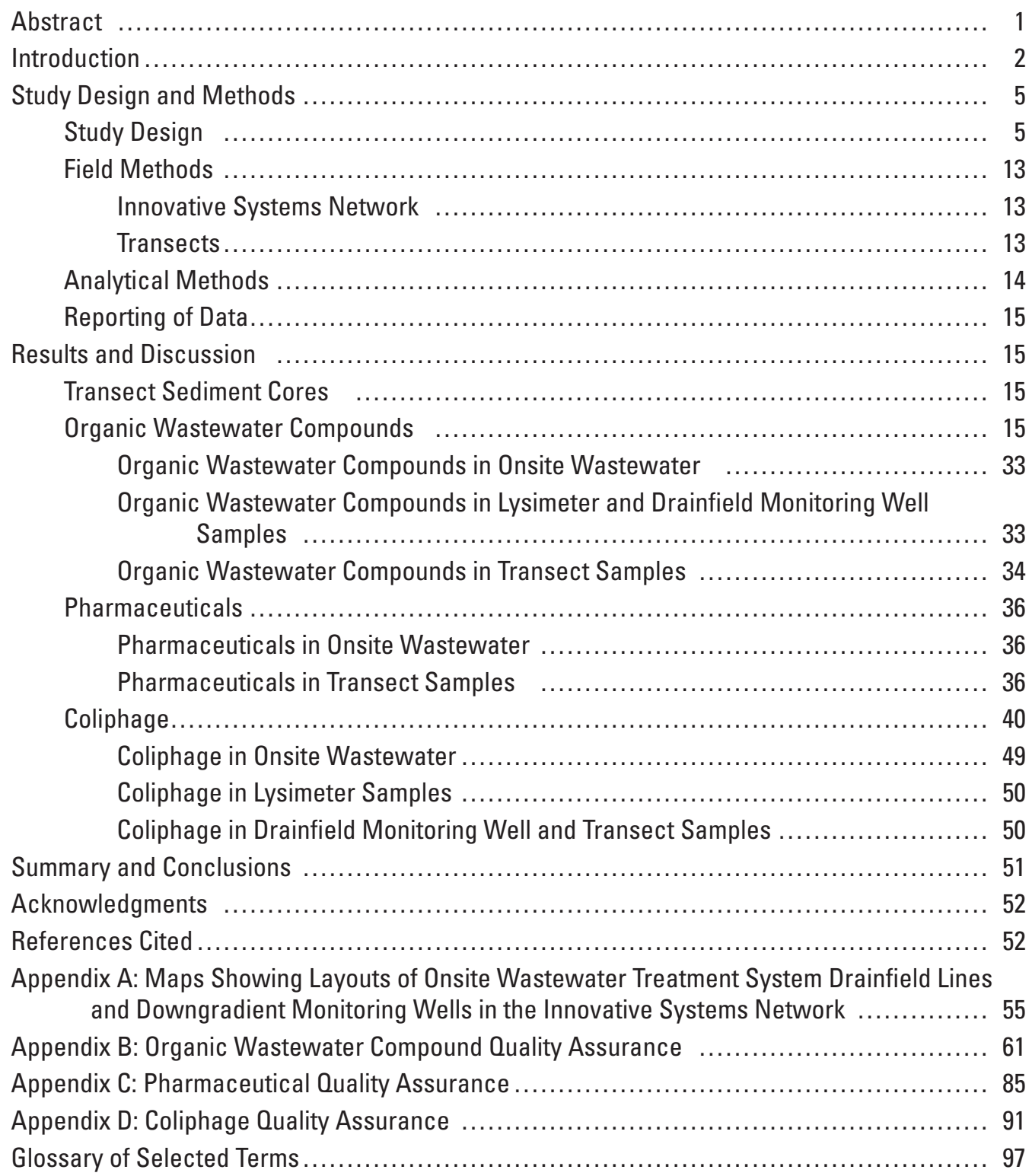




\section{Figures}

Figure 1. Map showing the La Pine, Oregon, study area............................. 4

Figure 2. Graph showing locations of transect wells at the Senior Center transect near La Pine,

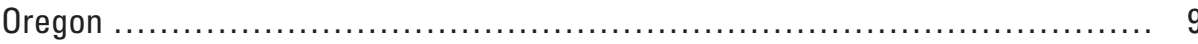

Figure 3. Graph showing locations of transect wells at the High Lakes Church transect near

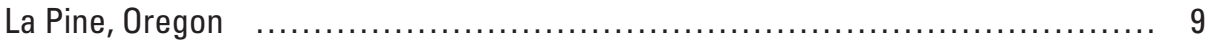

Figure 4. Graph showing locations of transect wells at the Pine Forest Road transect near La Pine, Oregon

Figure 5. Graph showing relation between chloride and nitrite-plus-nitrate concentrations for the three transects near La Pine, Oregon, 2003

Figure 6. Graph showing statistical distribution of coliphage concentrations in septic tank effluent samples from innovative systems network near La Pine, Oregon, 2003

\section{Tables}

Table 1. Traditional and innovative onsite wastewater treatment systems sampled in the vicinity of La Pine, Oregon, 2003.

Table 2. Locations of drainfield monitoring wells in relation to onsite wastewater treatment system drainfield lines, La Pine, Oregon........................................

Table 3. Well construction information for transect wells, La Pine, Oregon ..................... 10

Table 4. Project samples analyzed for organic wastewater compounds, pharmaceuticals, and coliphage, La Pine, Oregon.

Table 5. Organic carbon content, particle size distribution, and lithology from transect cores from the La Pine aquifer, La Pine, Oregon, 2003.

Table 6. Concentrations of organic wastewater compounds in water from traditional and innovative onsite wastewater treatment systems, downgradient lysimeters, and downgradient ground water, La Pine, Oregon, 2003.

Table 7. Concentrations of organic wastewater compounds in ground water along transects, La Pine, Oregon, 2003.

Table 8. Organic wastewater compounds detected in more than 90 percent of 21 onsite wastewater samples, La Pine, Oregon, 2003.

Table 9. Organic wastewater compounds detected in ground-water samples from

(a) drainfield monitoring wells, innovative on-site wastewater treatment system network, and (b) transect wells, La Pine, Oregon, 2003.

Table 10. Concentrations of pharmaceuticals in ground water along Senior transect, La Pine, Oregon, 2003.

Table 11. Pharmaceuticals tentatively identified during analysis of ground-water samples for organic wastewater compounds, La Pine, Oregon, 2003.

Table 12. Coliphage data for water from traditional and innovative onsite wastewater treatment systems, downgradient lysimeters, and downgradient ground water, La Pine, Oregon, 2003

Table 13. Coliphage data for water from transect wells, La Pine, Oregon, 2003 


\section{Conversion Factors}

\begin{tabular}{|c|c|c|}
\hline Multiply & By & To obtain \\
\hline \multicolumn{3}{|c|}{ Length } \\
\hline inch (in.) & 25.4 & millimeter (mm) \\
\hline inch (in.) & 2.54 & centimeter $(\mathrm{cm})$ \\
\hline foot $(\mathrm{ft})$ & 0.3048 & meter $(\mathrm{m})$ \\
\hline mile (mi) & 1.609 & kilometer $(\mathrm{km})$ \\
\hline \multicolumn{3}{|c|}{ Area } \\
\hline square mile $\left(\mathrm{mi}^{2}\right)$ & 2.590 & square kilometer $\left(\mathrm{km}^{2}\right)$ \\
\hline
\end{tabular}

Temperature in degrees Celsius $\left({ }^{\circ} \mathrm{C}\right)$ can be converted to degrees Fahrenheit $\left({ }^{\circ} \mathrm{F}\right)$ as follows:

$$
{ }^{\circ} \mathrm{F}=\left(1.8 x^{\circ} \mathrm{C}\right)+32
$$

Vertical coordinate information is referenced to the National Geodetic Vertical Datum of 1929 (NGVD 29).

Horizontal coordinate information is referenced to the North American Datum of 1983 (NAD 83). UTM refers to the Universal Transverse Mercator coordinate system; zone 10 in this report.

Altitude, as used in this report, refers to distance above the vertical datum.

Specific conductance is given in microsiemens per centimeter at 25 degrees Celsius $(\mu \mathrm{S} / \mathrm{cm}$ at $\left.25^{\circ} \mathrm{C}\right)$.

Concentrations of chemical constituents in water are given either in milligrams per liter $(\mathrm{mg} / \mathrm{L})$ or micrograms per liter $(\mu \mathrm{g} / \mathrm{L})$. One liter $(\mathrm{L})$ is equal to 1,000 milliliters $(\mathrm{mL})$. Nitrate concentrations are reported in units of milligrams nitrogen per liter $(\mathrm{mg} \mathrm{N} / \mathrm{L})$, in contrast to the occasionally seen units of milligrams nitrate per liter $\left(\mathrm{mg} \mathrm{NO}_{3}^{-} / \mathrm{L}\right)$. 


\title{
Organic Wastewater Compounds, Pharmaceuticals, and Coliphage in Ground Water Receiving Discharge from Onsite Wastewater Treatment Systems near La Pine, Oregon: Occurrence, and Implications for Transport
}

\author{
By Stephen R. Hinkle, Rodney J. Weick, Jill M. Johnson, Jeffery D. Cahill, Steven G. Smith, and \\ Barbara J. Rich
}

\section{Abstract}

The occurrence of organic wastewater compounds (components of "personal care products" and other common household chemicals), pharmaceuticals (human prescription and nonprescription medical drugs), and coliphage (viruses that infect coliform bacteria, and found in high concentrations in municipal wastewater) in onsite wastewater (septic tank effluent) and in a shallow, unconfined, sandy aquifer that serves as the primary source of drinking water for most residents near La Pine, Oregon, was documented. Samples from two types of observation networks provided basic occurrence data for onsite wastewater and downgradient ground water. One observation network was a group of 28 traditional and innovative (advanced treatment) onsite wastewater treatment systems and associated downgradient drainfield monitoring wells, referred to as the "innovative systems network." The drainfield monitoring wells were located adjacent to or under onsite wastewater treatment system drainfield lines. Another observation network, termed the "transect network," consisted of 31 wells distributed among three transects of temporary, stainless-steelscreened, direct-push monitoring wells installed along three plumes of onsite wastewater. The transect network, by virtue of its design, also provided a basis for increased understanding of the transport of analytes in natural systems.

Coliphage were frequently detected in onsite wastewater. Coliphage concentrations in onsite wastewater were highly variable, and ranged from less than 1 to 3,000,000 plaque forming units per 100 milliliters. Coliphage were occasionally detected (eight occurrences) at low concentrations in samples from wells located downgradient from onsite wastewater treatment system drainfield lines. However, coliphage concentrations were below method detection limits in replicate or repeat samples collected from the eight sites. The consistent absence of coliphage detections in the replicate or repeat samples is interpreted to indicate that the detections reported for groundwater samples represented low-level field or laboratory contamination, and it would appear that coliphage were effectively attenuated to less than $1 \mathrm{PFU} / 100 \mathrm{~mL}$ over distances of several feet of transport in the La Pine aquifer and (or) overlying unsaturated zone.

Organic wastewater compounds were frequently detected in onsite wastewater. Of the 63 organic wastewater compounds in the analytical schedule, 45 were detected in the 21 samples of onsite wastewater. Concentrations of organic wastewater compounds reached a maximum of $1,300 \mu \mathrm{g} / \mathrm{L}$ (p-cresol). Caffeine was detected at concentrations as high as $320 \mu \mathrm{g} / \mathrm{L}$. Fourteen of the 45 compounds were detected in more than 90 percent of onsite wastewater samples. Fewer (nine) organic wastewater compounds were detected in ground water, despite the presence of nitrate and chloride likely from onsite wastewater sources. The nine organic wastewater compounds that were detected in ground-water samples were acetyl-hexamethyl-tetrahydro-naphthalene (AHTN), caffeine, cholesterol, hexahydrohexamethyl-cyclopentabenzopyran, $\mathrm{N}, \mathrm{N}$-diethyl-meta-toluamide (DEET), tetrachloroethene, tris (2-chloroethyl) phosphate, tris (dichloroisopropyl) phosphate, and tributyl phosphate. Frequent detection of household-chemical type organic wastewater compounds in onsite wastewater provides evidence that some of these organic wastewater compounds may be useful indicators of human waste effluent dispersal in some hydrologic environments. The occurrence of organic wastewater compounds in ground water downgradient from onsite wastewater treatment systems demonstrates that a subgroup of organic wastewater compounds is transported in the La Pine aquifer. The consistently low concentrations (generally less than $1 \mu \mathrm{g} / \mathrm{L}$ ) of organic wastewater compounds in water samples collected from wells located no more than 19 feet from drainfield lines indicates that the reactivity (sorption, degradation) of this suite of organic wastewater compounds may limit their usefulness as tracers of onsite wastewater discharged into aquifers.

Ground-water samples from 1 of the 3 ground-water transects, along with 1 sample from the onsite wastewater treatment system associated with that transect, were analyzed for a suite of 18 pharmaceuticals. Eight pharmaceuticals were detected in the onsite wastewater, at concentrations up to about 
$120 \mu \mathrm{g} / \mathrm{L}$ (acetaminophen). In downgradient ground-water samples, sulfamethoxazole (an antibacterial), acetaminophen (an analgesic), and caffeine (a stimulant, and not a medical drug) each were detected once, at concentrations between $0.10 \mu \mathrm{g} / \mathrm{L}$ and $0.18 \mu \mathrm{g} / \mathrm{L}$-typical of the range of concentrations observed in other studies of wastewater-impacted ground water. In addition to the readily identified pharmaceuticals, two pharmaceuticals - the anticonvulsant drugs primidone and phenobarbitol-were tentatively identified in three groundwater samples from one nest of wells at another transect. Tentative identification of primidone and phenobarbitol occurred during analysis of ground-water samples for organic wastewater compounds; chromatogram peaks not associated with the target organic wastewater compounds were observed and the mass spectra of the unidentified compounds were matched to known mass spectra in a mass spectral reference library. Estimated concentrations reached as high as $12 \mu \mathrm{g} / \mathrm{L}$ (primidone). As was the case with organic wastewater compounds, the pharmaceutical occurrence data indicate that some pharmaceuticals may be useful indicators of the presence of human waste in the environment, and a subset of pharmaceuticals is transported to ground water from onsite wastewater treatment systems.

\section{Introduction}

Approximately 25 percent of U.S. residents use onsite wastewater treatment systems (usually septic tanks) for human wastewater dispersal and treatment (U.S. Environmental Protection Agency, 1980; Gerba, 2000a, p. 505). Use of onsite wastewater treatment systems has been accompanied by a substantial body of research on processes controlling onsite wastewater quality and on processes of transport operating in aquifers receiving discharge from onsite wastewater treatment systems to determine the fate of the compounds. Much of this work has focused on nitrogen, large amounts of which are released to aquifers by dispersal of onsite wastewater. Once released into permeable geologic materials and oxidized to nitrate, it is highly mobile as long as redox conditions remain oxidizing. The great mobility of nitrate in aquifers is important because nitrate is a contaminant of health concern. Furthermore, understanding the transport of nitrate derived from onsite wastewater treatment systems can lead to increased understanding of the transport of other contaminants derived from onsite wastewater treatment systems.

The transport and fate of other contaminants associated with onsite wastewater have received less study than that of nitrate. Organic wastewater compounds (components of "personal care products" and other common household chemicals) and pharmaceuticals (medical drugs), often termed "emerging contaminants," have only begun to attract attention in recent years as the analytical capability to detect and quantify these compounds at the microgram-per-liter and sub-microgramper-liter concentration range that is relevant for environmental occurrence investigation has been developed (Daughton, 2001; Kolpin and others, 2002). Studies in the last 5 to 10 years have begun characterizing the environmental distribution of some of these compounds. New laboratory methods have been or are under development at several U.S. Geological Survey (USGS) research laboratories and at the USGS National Water Quality Laboratory (NWQL) (Denver, Colorado) to provide the analytical capabilities to measure concentrations of suites (schedules) of organic wastewater compounds, pharmaceuticals, antibiotics, hormones, and other related compounds, the occurrence of which are of concern in the aquatic environment. Many organic wastewater compounds and pharmaceuticals have endocrine-disrupting properties or are otherwise biologically active, even at microgram-per-liter and sub-microgram-per-liter concentrations (Daughton and Ternes, 1999; Zaugg and others, 2002; Masters and others, 2004). A review of known and potential effects of many organic wastewater compounds and pharmaceuticals on human and ecologic health is provided by Daughton and Ternes (1999). A broad range of information about organic wastewater compounds and pharmaceuticals, including analytical methods, environmental occurrence, sources and source pathways, transport and fate through the environment, potential ecologic effects, and on-line publications, can be found on the World Wide Web at http://toxics.usgs.gov/regional/emc/index.html .

Virus occurrence and transport in ground water have been more thoroughly studied than have organic wastewater compound and pharmaceutical occurrence and transport because of the somewhat greater availability of analytical methods, and because of the longstanding understanding of the potential health risk associated with their presence (e.g., Keswick and Gerba, 1980). Many studies of virus transport in ground water have been of viruses originating from municipal wastewater and sludge (e.g., Yanko and others, 1999), which tend to be associated with artificially high recharge rates, or have been studies in which laboratory-grown viruses are directly injected into the aquifer and used as tracers (e.g., Bales and others, 1995). Studies of naturally occurring viruses from onsite wastewater treatment systems in relatively undisturbed, natural settings have been few, although the study by DeBorde and others (1998) is a notable exception.

The work presented in this report arose from a perceived need to better characterize the occurrence of organic wastewater compounds, pharmaceuticals, and naturally occurring viruses in onsite wastewater and in downgradient ground water. Although this study was primarily an occurrence survey, it included a ground-water-transect component to provide a basis for increased understanding of the transport of these analytes in relatively undisturbed, natural systems.

The organic wastewater compounds of interest were a suite of 63 organic compounds typically found in personal care and household products and in domestic and industrial wastewater, including caffeine, cholesterol, menthol, camphor, cotinine (a nicotine metabolite), detergent metabolites, antimicrobial agents, disinfectants, antioxidants, and compounds originating from deodorants and fragrances. Information about 
sources and uses of these organic wastewater compounds can be found in Zaugg and others (2002) (available on the World Wide Web at http://nwql.usgs.gov/Public/pubs/WRIR01-4186. html). The pharmaceuticals of interest were a group of human prescription and nonprescription drugs and drug metabolites.

The viruses that were chosen for study were F-specific and somatic coliphage. Coliphage are viruses that infect coliform bacteria; they are found in high concentrations in municipal wastewater (IAWPRC Study Group on Health Related Water Microbiology, 1991). F-specific coliphage infect the F-pili of male strains of coliform bacteria. Somatic coliphage infect the outer cell wall of coliform bacteria. F-specific coliphage replicate in warm-blooded hosts; somatic coliphage replicate in warm-blooded hosts but also may replicate in natural waters (IAWPRC Study Group on Health Related Water Microbiology, 1991; Handzel and others, 1993). However, both F-specific and somatic coliphage are generally found at much higher concentrations in municipal sewage than in unpolluted water (IAWPRC Study Group on Health Related Water Microbiology, 1991). Hence, coliphage may have the potential to serve as indicators of onsite wastewater. Understanding coliphage survival and transport may eventually lead to an improved understanding of enteric virus survival and transport, but the degree to which coliphage survival and transport correlates to enteric virus survival and transport has yet to be established (Francy and others, 2000).

The location for this study was the rural-residential community of La Pine, in Oregon's upper Deschutes Basin (fig. 1). Study area geology consists of up to about 1,300 feet of Quaternary alluvial and lacustrine deposits in a structural basin of Quaternary and Tertiary basalt, andesite, vent deposits, and pyroclastic rocks (Gannett and others, 2001; Lite and Gannett, 2002). Sand is common in the uppermost 100 feet of the basin. This sand is the primary aquifer in the La Pine area, and most domestic wells are screened within 50 feet of land surface. This aquifer serves as the sole source of drinking water for residents of the La Pine area. This aquifer also serves as the receptor of onsite wastewater for most residents, who live in private residences scattered throughout the study area. Most homes are unsewered, and onsite wastewater treatment systems are used to disperse wastewater. As of 1999, 5,185 homes in the study area were served by onsite wastewater treatment systems (D.S. Morgan, U.S. Geological Survey, written commun., 2005). Most houses in this semiarid study area are surrounded by semidesert landscaping, and agricultural activities are essentially nonexistent (Oregon Department of Environmental Quality, 1994). Of the Deschutes County portion of the study area (Deschutes County represents $83 \%$ of the study area; fig. 1), 8.7 square miles (4.2\% of the Deschutes County portion of the study area) was zoned tax-deferred (active) agricultural in 2004 (Tim Berg, Deschutes County Community Development Department, written commun., 2004). Of these 8.7 square miles, $72 \%$ was nonirrigated. Agriculture in the La Pine area is essentially all pasture and hay, the climate (altitude about 4,200 feet above sea level) being too cold to allow more intensive types of agriculture such as grains or row crops. Fertilizer use is inappreciable, and septic tank effluent is the only significant anthropogenic source of nitrogen to study area ground water (Century West Engineering Corporation, 1982). Concentrations of nitrite-plus-nitrate (henceforth, "nitrate") exceeding the Oregon Department of Environmental Quality (ODEQ) action level of $7 \mathrm{mg}$ as nitrogen/L (7 mg N/L) and the U.S. Environmental Protection Agency (USEPA) Maximum Contaminant Level of $10 \mathrm{mg} \mathrm{N} / \mathrm{L}$ are found in the aquifer and have largely been attributed to the widespread use of onsite wastewater treatment systems (Oregon Department of Environmental Quality, 1994; Hinkle and others, 2002). ODEQ and Deschutes County Environmental Health Division (DCEHD) have been evaluating the effects of onsite wastewater treatment systems on ground-water quality, with an emphasis on nitrate and select, other wastewater contaminants such as fecal indicator bacteria (fecal coliform and E. coli). These agencies have been measuring the long-term (period of years) performance of a variety of traditional and innovative (advanced-treatment) onsite wastewater treatment systems in the study area (Oregon Department of Environmental Quality, 2004; Deschutes County, Environmental Health Division, no date). The USGS has been collaborating with ODEQ and DCEHD to generate a quantitative understanding of nitrate transport and fate in the La Pine aquifer (Hinkle and others, 2002) and to produce a numerical nitrate transport model that can be used in a predictive manner to evaluate the effects of various land-use and wastewater-treatment options on groundwater quality in the La Pine area (Morgan and others, 2002). The presence of a network of monitored onsite wastewater treatment systems distributed throughout a study area with physically and chemically well-defined local and regional ground-water flow systems made the La Pine study area an ideal environment for a study of household-type organic wastewater compounds, pharmaceuticals, and wastewaterassociated viruses.

This report (1) documents basic physical and chemical data of organic wastewater compound, pharmaceutical, and coliphage occurrence in ground water affected by dispersal of onsite wastewater in the vicinity of La Pine, Oregon, and (2) provides a general discussion of the occurrence of organic wastewater compounds, pharmaceuticals, and coliphage; variability at the source and in ground water; and resultant implications for transport of these analytes in a sandy aquifer. The primary emphasis of this report is on describing the occurrence of organic wastewater compounds, pharmaceuticals, and coliphage in onsite wastewater and in downgradient ground water. The quality of ground water near onsite wastewater treatment systems was characterized, but drinking-water wells were not sampled. Although process-based understanding of transport and fate of organic wastewater compounds, pharmaceuticals, and coliphage is needed, the study described in this report was more on the order of a reconnaissance occurrence effort; robust transport and fate characterization was beyond the scope of this work. 


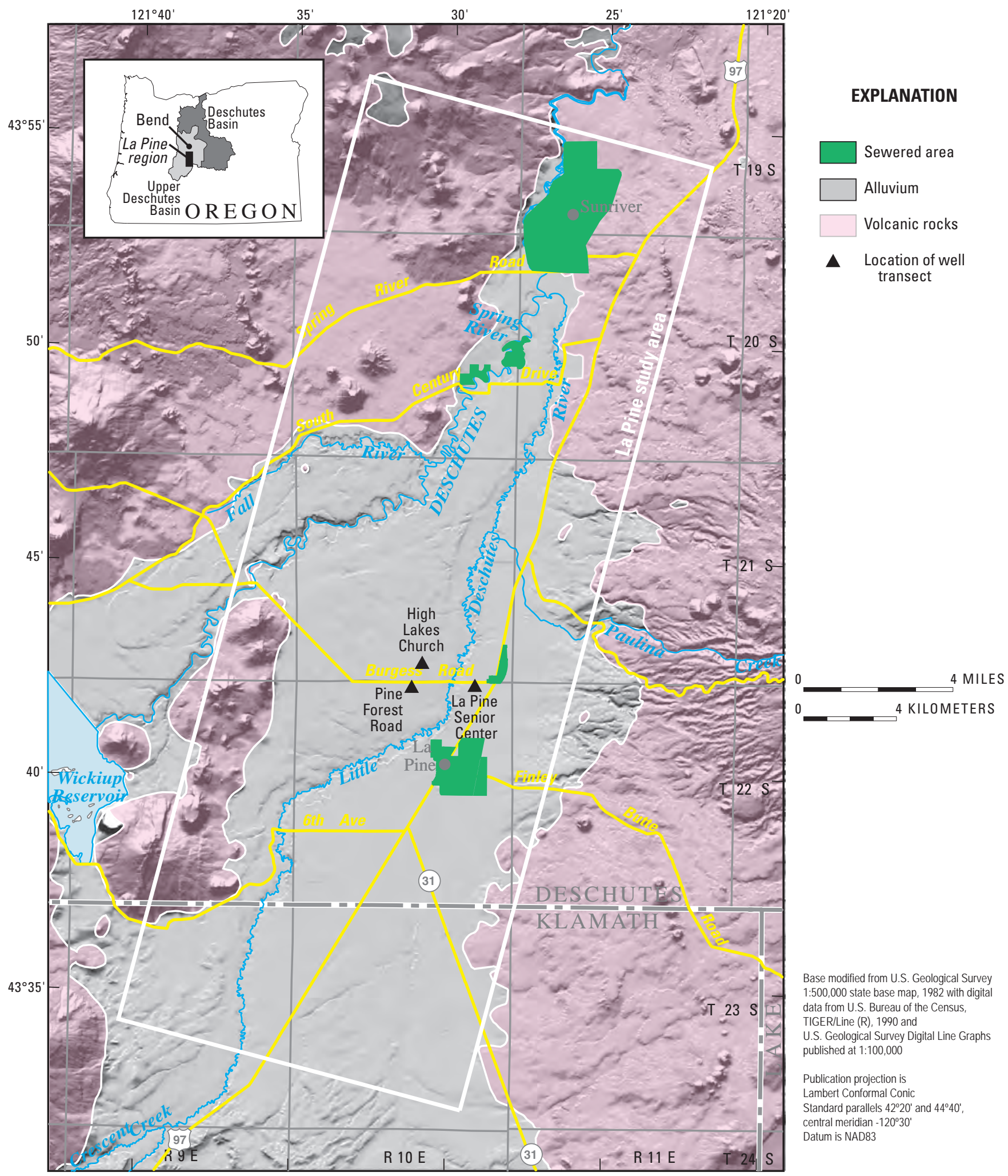

Figure 1. The La Pine, Oregon, study area. 


\section{Study Design and Methods}

\section{Study Design}

The study design was built around two types of observation networks. These two observation networks focused on different aspects of onsite wastewater in the environment.

One observation network was a group of onsite wastewater treatment systems that had already been installed by ODEQ and DCEHD to evaluate the effectiveness of various traditional and innovative onsite wastewater treatment systems. This observation network is called the innovative systems network. Twenty-eight onsite wastewater treatment systems -2 of each of 3 traditional and 2 of each of 11 innovative systems-were monitored for the present study (table 1). Each onsite wastewater treatment system was instrumented with sampling access. Two types of traditional onsite wastewater treatment systems - standard (gravity) and pressure-were instrumented to allow sampling at the septic tank, at a lysimeter beneath a drainfield line in native sediment, and at a downgradient monitoring well located adjacent to the drainfield lines (drainfield monitoring well) (Appendix A). A third type of traditional onsite wastewater treatment system — sand filter-was instrumented at the septic tank, at the bottom of the sand filter, and at a drainfield monitoring well (Appendix A). Eleven types of innovative onsite wastewater treatment systems were instrumented to allow sampling at the septic tank (except for the Amphidrome systems, which were not instrumented at the septic tank), at a location immediately downgradient from the additional (innovative) onsite treatment, and at a drainfield monitoring well (Appendix A). One lysimeter associated with the innovative system network onsite wastewater treatment systems (at one of the NAYADIC systems) also was sampled. All onsite wastewater treatment systems were located in alluvium in the Deschutes County portion of the La Pine study area (fig. 1). Descriptions of the relative locations of the sampling locations within the onsite wastewater treatment systems and in downgradient ground water are described in table 2.

The second observation network was composed of temporary, stainless-steel-screened, direct-push monitoring wells installed along plumes of onsite wastewater. In this study, these groups of monitoring wells are called the transect wells. The use of transects of monitoring wells allows sampling locations to be placed in such a manner that evolution of groundwater chemistry can be observed (the "space-for-time" concept inherent in well transects). An additional benefit of the transects is that monitoring wells provide access to ground water without significantly altering the natural hydraulic gradient (a concern with using water supply wells), thus providing insight into aquifer vulnerability largely independent of pumping perturbations. Plumes were assumed to flow away from onsite wastewater treatment system drainfield lines in the hydraulically downgradient direction (hydraulic gradients were based upon an aquifer-scale potentiometric map provided by D.S.
Morgan, U.S. Geological Survey, written commun., 2003), and were identified on the basis of field nitrate testing of purged ground water using semiquantitative test strips and subsequently confirmed with routine laboratory nitrate analysis. Ground-water flow in the vicinity of the transects is generally towards the Little Deschutes River (fig. 1), with ground-water fluxes from the aquifer occurring primarily by a combination of direct discharge to the Little Deschutes River and evapotranspiration in the near-stream environment where the water table is shallow. Three transects were installed. All three transects were in parts of the study area believed to be typical of the study area as a whole. They consisted of 11 wells (plus an additional 3 that were installed for the purposes of resampling, using identical procedures) at the La Pine Senior Center (henceforth, Senior transect), 9 at the High Lakes Christian Church (henceforth, High Lakes Church transect), and 11 in the public right-of-way along Pine Forest Road in a plume identified in a previous investigation (henceforth, Pine transect). The onsite wastewater treatment system at the La Pine Senior Center also was sampled as part of this work because it was the presumed source of the Senior transect plume. In addition to installation of temporary wells, one set of split spoon sediment cores was collected at each transect. Sediment was logged in the field, and then subsampled for particle-size analysis and organic carbon concentration.

The three transect sites were chosen primarily on the basis of available access for drilling. All three systems were mature systems. The church system was installed in 1984, the senior center system, 1990, and the closest residential system lying directly upgradient from the Pine transect, 1985. Onsite wastewater treatment systems serving the church and the senior center may have characteristics that differ from those serving homes. For example, church and senior center waste may have higher urine-to-solids ratios than would be found in typical residential systems. However, the senior center provides food service, and the church holds week-long day camp programs. Such activities provide for a measure of solids in the onsite wastewater.

Maps showing the locations of the transect wells are shown in figures 2, 3, and 4, and well construction data are shown in table 3 . The absence of physical features on figures 2,3 , and 4 are a reflection of the relatively featureless terrain at the transect sites; no streams, wetlands, or other notable physical features were omitted from the figures. As is evident in figures 2,3, and 4, there was a bias in the distribution of transect wells, with a greater density of wells near drainfield lines (Senior and High Lakes Church transects) or the upgradient edge of the transect where drainfield line locations were not mapped (Pine transect). This bias was intentional, the aim being to provide a greater level of characterization in the upgradient region of the transects. 
Table 1. Traditional and innovative onsite wastewater treatment systems sampled in the vicinity of La Pine, Oregon, 2003.

[Additional technical information on these systems can be obtained from the La Pine National Demonstration Project Web site at: http://marx.deschutes.org/deq/lapineindex.htm]

\begin{tabular}{|c|c|c|}
\hline $\begin{array}{l}\text { Name of } \\
\text { onsite waste- } \\
\text { water treat- } \\
\text { ment system }\end{array}$ & Type of treatment & Treatment process \\
\hline Standard & Natural & House $\rightarrow$ septic tank $\rightarrow$ gravity feed to drainfield \\
\hline Pressure & Natural & House $\rightarrow$ septic tank $\rightarrow$ pressurized drainfield \\
\hline Sand Filter & Natural & $\begin{array}{l}\text { House } \rightarrow \text { septic tank } \rightarrow \text { pressurized drainfield lines in a sand filter; sand filter built above natural } \\
\text { ground level, discharge to soil beneath filter }\end{array}$ \\
\hline $\begin{array}{l}\text { AdvanTex® } \\
(\mathrm{AX}-20)\end{array}$ & Trickling/packed bed filter & House $\rightarrow$ septic tank $\rightarrow$ AX-20 filter $\rightarrow$ recirculate to septic tank $\rightarrow$ drip distribution field \\
\hline Amphidrome ${ }^{\circledR}$ & Sequencing batch reactor & $\begin{array}{l}\text { House } \rightarrow \operatorname{tank} 1 \rightarrow \text { reactor (aeration) } \rightarrow \text { tank } 2 \rightarrow \text { reactor }(\text { backwash) } \rightarrow \text { tank } 1 \rightarrow \text { reactor } \\
\quad(\text { aeration }) \rightarrow \operatorname{tank} 2 \rightarrow \text { gravity drainfield }\end{array}$ \\
\hline Biokreisel@ & Rotating biological contactor & $\begin{array}{l}\text { House } \rightarrow \text { septic tank } \rightarrow \text { rotating biological contactor } \rightarrow \text { recirculate through septic tank } \rightarrow \text { gravel } \\
\quad \text { (polishing) filter } \rightarrow \text { pressurized drainfield }\end{array}$ \\
\hline EnviroServer & Aeration & $\begin{array}{l}\text { House } \rightarrow \text { primary clarifier } \rightarrow \text { aeration chamber } \rightarrow \text { secondary clarifier } \rightarrow \text { recirculate to primary } \\
\quad \text { clarifier } \rightarrow \text { drip distribution field }\end{array}$ \\
\hline FAST® & $\begin{array}{l}\text { Forced aeration/attached } \\
\text { growth media }\end{array}$ & $\begin{array}{l}\text { House } \rightarrow \text { primary settling chamber } \rightarrow \text { forced aeration/ attached growth media } \rightarrow \text { pressure } \\
\text { drainfield }\end{array}$ \\
\hline NAYADIC & Aeration & House $\rightarrow$ septic tank $\rightarrow$ aeration $\rightarrow$ recirculate to septic tank $\rightarrow$ pressure drainfield \\
\hline NiteLess & Aeration with carbon source & $\begin{array}{l}\text { House } \rightarrow \text { primary clarification } \rightarrow \text { aeration } \rightarrow \text { secondary settling } \rightarrow \text { added carbon } \rightarrow \\
\text { pressure drainfield }\end{array}$ \\
\hline NITREX $^{\mathrm{TM}}$ & $\begin{array}{l}\text { Packed bed filter with carbon } \\
\text { source }\end{array}$ & House $\rightarrow$ septic tank $\rightarrow$ lined sand filter $\rightarrow$ packed bed filter (carbon source) $\rightarrow$ pressure drainfield \\
\hline Puraflo® & Packed bed filter & $\begin{array}{l}\text { House } \rightarrow \text { septic tank } \rightarrow \text { pump tank } \rightarrow \text { packed bed filter } \rightarrow \text { recirculate to septic tank } \rightarrow \text { pump tank } \\
\quad \rightarrow \text { discharge portion of effluent to soil beneath filter }\end{array}$ \\
\hline Wert B & $\begin{array}{l}\text { Trench with packed bed filter } \\
\text { and carbon }\end{array}$ & $\begin{array}{l}\text { House } \rightarrow \text { septic tank } \rightarrow \text { drip irrigation } \rightarrow \text { lined trench } \rightarrow \text { lined gravel trench with added carbon } \rightarrow \\
\quad \text { discharge to sidewall }\end{array}$ \\
\hline Dyno $2^{\mathrm{TM}}$ & Attached growth media & $\begin{array}{l}\text { House } \rightarrow \text { septic tank } \rightarrow \text { chamber for influent filter/attached growth media } \rightarrow \text { pump chamber } \rightarrow \\
\text { gravel filter/wetland } \rightarrow \text { recirculate to influent filter/attached growth media } \rightarrow \text { pressurized } \\
\text { drainfield }\end{array}$ \\
\hline
\end{tabular}


Table 2. Locations of drainfield monitoring wells in relation to onsite wastewater treatment system drainfield lines, La Pine, Oregon.

[Lateral distances derived from Appendix A; DFMW, drainfield monitoring well; type of onsite wastewater treatment system, see descriptions in table 1; Date as year, month, day (YYYYMMDD)]

\begin{tabular}{|c|c|c|c|c|c|c|c|c|c|c|}
\hline $\begin{array}{c}\text { Type of onsite } \\
\text { wastewater } \\
\text { treatment system }\end{array}$ & Station number & $\begin{array}{c}\text { Date on } \\
\text { which onsite } \\
\text { wastewater } \\
\text { treatment system } \\
\text { was first sampled }\end{array}$ & $\begin{array}{l}\text { Date on which first } \\
\text { coliphage sample } \\
\text { was collected }\end{array}$ & $\begin{array}{l}\text { Depth to water, } \\
\text { measured } \\
\text { at time of } \\
\text { collection of } \\
\text { first coliphage } \\
\text { sample } \\
\text { (feet) }\end{array}$ & $\begin{array}{l}\text { Depth to top } \\
\text { of screen } \\
\text { (feet) }\end{array}$ & $\begin{array}{c}\text { Penetration of } \\
\text { top of screen } \\
\text { below water table } \\
\text { (negative number } \\
\text { indicates screened } \\
\text { above water table) } \\
\text { (feet) }\end{array}$ & $\begin{array}{c}\text { Depth to } \\
\text { bottom of } \\
\text { screen } \\
\text { (feet) }\end{array}$ & $\begin{array}{l}\text { Lateral } \\
\text { distance from } \\
\text { closest part of } \\
\text { drainfield line } \\
\text { to DFMW } \\
\text { (feet) }\end{array}$ & $\begin{array}{l}\text { Lateral } \\
\text { distance from } \\
\text { center of } \\
\text { drainfield to } \\
\text { DFMW } \\
\text { (feet) }\end{array}$ & $\begin{array}{c}\text { Lateral } \\
\text { distance from } \\
\text { farthest part } \\
\text { of drainfield } \\
\text { line to DFMW } \\
\text { (feet) }\end{array}$ \\
\hline Standard & 434207121324601 & 20010326 & 20030414 & 27.47 & 27 & 0 & 33 & 8 & 20 & 55 \\
\hline Standard & 434236121310501 & 20010305 & 20030604 & 15.07 & 14 & -1 & 20 & 10 & 33 & 65 \\
\hline Pressure & 434247121305501 & 20010305 & 20030514 & 11.53 & 12 & 0 & 15 & 3 & 13 & 51 \\
\hline Pressure & 434248121295901 & 20011008 & 20030407 & 16.60 & 17 & 0 & 20 & 7 & 22 & 49 \\
\hline Sand Filter & 434347121293901 & 20010117 & 20030414 & 15.26 & 11 & -4 & 17 & 0 & 3 & 19 \\
\hline Sand Filter & 434741121273101 & 20001113 & 20030407 & 9.49 & 9 & 0 & 12 & 0 & 11 & 27 \\
\hline AdvanTex (AX-20) & 434652121273001 & 20020123 & 20030414 & 11.18 & 10 & -1 & 16 & 5 & 10 & 29 \\
\hline AdvanTex (AX-20) & 434536121291201 & 20020108 & 20030604 & 13.00 & 12 & -1 & 15 & 19 & 25 & 42 \\
\hline Amphidrome & 434011121314601 & 20020730 & 20030423 & 9.35 & 9 & 0 & 15 & 8 & 16 & 35 \\
\hline Amphidrome & 434243121290101 & 20021009 & 20030423 & 14.94 & 13 & -2 & 19 & 12 & 37 & 63 \\
\hline Biokreisel & 434226121293301 & 20010319 & 20030414 & 8.64 & 7 & -2 & 10 & 4 & 34 & 53 \\
\hline Biokreisel & 434727121273701 & 20010103 & 20030604 & 5.88 & 6 & 0 & 9 & 8 & 36 & 60 \\
\hline EnviroServer & 434836121271101 & 20010730 & 20030423 & 5.46 & 6 & 1 & 9 & 6 & 13 & 51 \\
\hline EnviroServer & 433855121300101 & 20010730 & 20030423 & 12.05 & 12 & 0 & 15 & 7 & 32 & 71 \\
\hline FAST & 434952121290601 & 20011217 & 20030407 & 5.80 & 8 & 2 & 11 & 5 & 28 & 53 \\
\hline FAST & 434437121295301 & 20010207 & 20030505 & 5.60 & 5 & -1 & 8 & 7 & 20 & 45 \\
\hline NAYADIC & 435016121284701 & 20011105 & 20030407 & 6.55 & 8 & 1 & 11 & 3 & 28 & 54 \\
\hline NAYADIC & 434713121274301 & 20011105 & 20030423 & 11.18 & 10 & -1 & 13 & 4 & 15 & 33 \\
\hline NiteLess & 434908121291201 & 20011217 & 20030519 & 6.04 & 7 & 1 & 10 & 7 & 13 & 34 \\
\hline
\end{tabular}


[Lateral distances derived from Appendix A; DFMW, drainfield monitoring well; type of onsite wastewater treatment system, see descriptions in table 1; Date as year, month, day (YYYYMMDD)]

\begin{tabular}{|c|c|c|c|c|c|c|c|c|c|c|}
\hline $\begin{array}{c}\text { Type of onsite } \\
\text { wastewater } \\
\text { treatment system }\end{array}$ & Station number & $\begin{array}{c}\text { Date on } \\
\text { which onsite } \\
\text { wastewater } \\
\text { treatment system } \\
\text { was first sampled }\end{array}$ & $\begin{array}{c}\text { Date on which first } \\
\text { coliphage sample } \\
\text { was collected }\end{array}$ & $\begin{array}{l}\text { Depth to water, } \\
\text { measured } \\
\text { at time of } \\
\text { collection of } \\
\text { first coliphage } \\
\text { sample } \\
\text { (feet) }\end{array}$ & $\begin{array}{l}\text { Depth to top } \\
\text { of screen } \\
\text { (feet) }\end{array}$ & $\begin{array}{l}\text { Penetration of top of } \\
\text { screen below water } \\
\text { table (negative } \\
\text { number indicates } \\
\text { screened above } \\
\text { water table) } \\
\text { (feet) }\end{array}$ & $\begin{array}{l}\text { Depth to } \\
\text { bottom of } \\
\text { screen } \\
\text { (feet) }\end{array}$ & $\begin{array}{l}\text { Lateral } \\
\text { distance from } \\
\text { closest part } \\
\text { of drainline to } \\
\text { DFMW } \\
\text { (feet) }\end{array}$ & $\begin{array}{l}\text { Lateral } \\
\text { distance from } \\
\text { center of } \\
\text { drainfield to } \\
\text { DFMW } \\
\text { (feet) }\end{array}$ & $\begin{array}{c}\text { Lateral } \\
\text { distance } \\
\text { from farthest } \\
\text { part of } \\
\text { drainline to } \\
\text { DFMW } \\
\text { (feet) } \\
\end{array}$ \\
\hline NiteLess & 434431121293501 & 20020108 & 20030604 & 8.33 & 11 & 3 & 14 & 6 & 22 & 46 \\
\hline NITREX & 434203121311201 & 20001226 & 20030505 & 9.18 & 5 & -4 & 11 & 4 & 12 & 45 \\
\hline NITREX & 433825121340001 & 20001226 & 20030519 & 10.57 & 8 & -3 & 14 & 7 & 16 & 41 \\
\hline Puraflo & 434010121325601 & 20011210 & 20030519 & 14.90 & 14 & -1 & 17 & 6 & 22 & 39 \\
\hline Puraflo & 434324121292601 & 20020402 & 20030604 & 10.33 & 10 & 0 & 16 & 6 & 23 & 40 \\
\hline Wert B & 434449121310201 & 20020827 & 20030514 & 27.56 & 27 & -1 & 33 & 8 & 18 & 39 \\
\hline Wert B & 434423121312901 & 20020827 & 20030514 & 29.98 & 28 & -2 & 34 & 4 & 28 & 55 \\
\hline Dyno2 & 433950121322901 & 20020122 & 20030514 & 9.14 & 9 & 0 & 12 & 5 & 11 & 46 \\
\hline Dyno2 & 434131121314301 & 20020128 & 20030505 & 12.95 & 12 & -1 & 15 & 11 & 19 & 54 \\
\hline
\end{tabular}




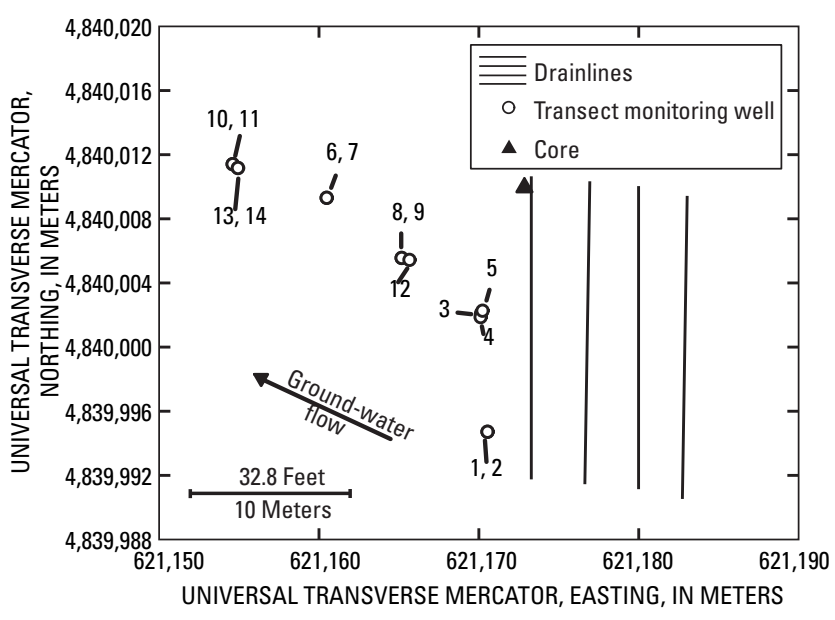

Figure 2. Locations of transect wells at the Senior Center transect near La Pine, Oregon. General direction of regional ground-water flow shown with arrow. Multiple wells at one location indicted by multiple identification numbers. Transect well 12 was drilled to resample transect well 9. Transect wells 13 and 14 were drilled to resample transect well 10.

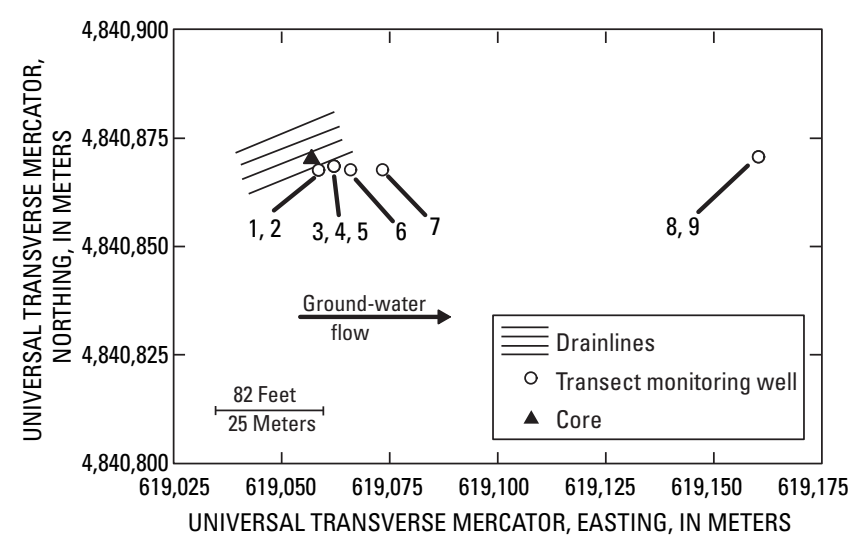

Figure 3. Locations of transect wells at the High Lakes Church transect near La Pine, Oregon. General direction of regional ground-water flow shown with arrow. Multiple wells at one location indicted by multiple identification numbers.

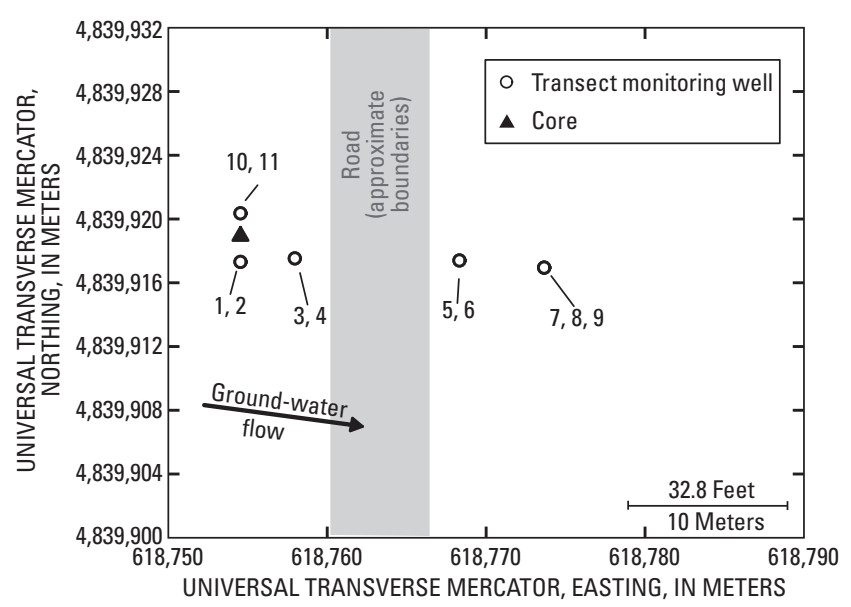

Figure 4. Locations of transect wells at the Pine Forest Road transect near La Pine, Oregon. General direction of regional ground-water flow shown with arrow. Multiple wells at one location indicted by multiple identification numbers.

Samples collected from both networks were analyzed for various wastewater analytes (table 4). Transect samples were analyzed for coliphage and organic wastewater compounds. Samples from the Senior transect were analyzed for pharmaceuticals. All transect samples also were analyzed for chloride and nitrate, which are useful as indicators of the presence of onsite wastewater, and in the case of chloride, can be used to estimate the proportion of onsite wastewater in ground-water samples.

Samples from the innovative systems network were analyzed for coliphage. A subset of samples was analyzed for organic wastewater compounds (20 septic tank samples, 5 lysimeter samples, and 20 downgradient monitoring wells). Coliphage sampling at the innovative systems network sites was done twice (spring 2003, and fall 2003); other samples were collected once. Most samples were also analyzed for chloride and selected nitrogen species.

The intentional well-placement bias inherent in the two observation networks allows for detailed characterization of the concentration distribution immediately or soon after recharge near wastewater sources. The characterization of concentrations proximal to sources allows evaluation of near-source attenuation processes representing the summed effects of sorption, biotic and abiotic degradation, and local dispersion. Downgradient longitudinal and transverse dispersion increases with distance traveled. The study of this scale of dispersion is beyond the scope of this work and is a limitation. 
[Elevation of static water level at Senior 10 and Senior 11, which are at the western edge of the gravel parking area, may be slightly elevated due to focused recharge from snow plowed from winter storms and piled at the western edge of the parking area; High 8 sampled 6/12/2003, but water level was slow to recover and hence measured 6/13/2003; Date as year, month, day (YYYYMMDD); -, no data]

\begin{tabular}{|c|c|c|c|c|c|c|c|c|c|c|}
\hline Site name & Station number & $\begin{array}{l}\text { Latitude } \\
\text { (NAD83) }\end{array}$ & $\begin{array}{l}\text { Longitude } \\
\text { (NAD83) }\end{array}$ & $\begin{array}{l}\text { UTM North } \\
\text { (NAD83) }\end{array}$ & $\begin{array}{l}\text { UTM East } \\
\text { (NAD83) }\end{array}$ & $\begin{array}{c}\text { Elevation of } \\
\text { land surface } \\
\text { (feet) }\end{array}$ & $\begin{array}{l}\text { Elevation } \\
\text { of top of } \\
\text { screened } \\
\text { interval } \\
\text { (feet) }\end{array}$ & $\begin{array}{c}\text { Elevation } \\
\text { of bottom } \\
\text { of screened } \\
\text { interval } \\
\text { (feet) }\end{array}$ & $\begin{array}{c}\text { Elevation of } \\
\text { static water } \\
\text { level } \\
\text { (feet) }\end{array}$ & $\begin{array}{l}\text { Date of static water } \\
\text { level measurement } \\
\text { (year/month/day) }\end{array}$ \\
\hline Senior Core & 434212121294200 & 434211.6 & 1212946.0 & 4840010.0 & 621172.9 & - & - & - & - & - \\
\hline Senior 1 & 434212121294201 & 434211.1 & 1212946.1 & 4839994.7 & 621170.5 & 4219.29 & 4202 & 4200 & 4207.90 & 20030429 \\
\hline Senior 2 & 434212121294202 & 434211.1 & 1212946.1 & 4839994.7 & 621170.5 & 4219.29 & 4199 & 4197 & 4207.85 & 20030429 \\
\hline Senior 3 & 434212121294203 & 434211.3 & 1212946.1 & 4840002.1 & 621170.1 & 4219.14 & 4200 & 4198 & 4207.71 & 20030430 \\
\hline Senior 4 & 434212121294204 & 434211.3 & 1212946.1 & 4840001.9 & 621170.1 & 4219.14 & 4198 & 4196 & 4207.70 & 20030430 \\
\hline Senior 5 & 434212121294205 & 434211.3 & 1212946.1 & 4840002.3 & 621170.2 & 4219.14 & 4202 & 4200 & 4207.91 & 20030430 \\
\hline Senior 6 & 434212121294206 & 434211.5 & 1212946.6 & 4840009.3 & 621160.5 & 4219.09 & 4202 & 4198 & 4207.47 & 20030501 \\
\hline Senior 7 & 434212121294207 & 434211.5 & 1212946.6 & 4840009.3 & 621160.5 & 4219.09 & 4198 & 4195 & 4207.41 & 20030501 \\
\hline Senior 8 & 434212121294208 & 434211.4 & 1212946.4 & 4840005.6 & 621165.2 & 4219.13 & 4202 & 4199 & 4207.61 & 20030501 \\
\hline Senior 9 & 434212121294209 & 434211.4 & 1212946.4 & 4840005.6 & 621165.2 & 4219.13 & 4197 & 4196 & 4207.56 & 20030501 \\
\hline Senior 10 & 434212121294210 & 434211.6 & 1212946.8 & 4840011.4 & 621154.6 & 4218.98 & 4202 & 4199 & 4207.59 & 20030502 \\
\hline Senior 11 & 434212121294211 & 434211.6 & 1212946.8 & 4840011.4 & 621154.6 & 4218.98 & 4196 & 4195 & 4207.61 & 20030502 \\
\hline Senior 12 & 434212121294212 & 434211.4 & 1212946.3 & 4840005.4 & 621165.7 & 4219.45 & 4197 & 4196 & 4207.49 & 20030618 \\
\hline Senior 13 & 434212121294213 & 434211.6 & 1212946.8 & 4840011.2 & 621154.9 & 4218.89 & 4201 & 4199 & - & 20030618 \\
\hline Senior 14 & 434212121294214 & 434211.6 & 1212946.8 & 4840011.2 & 621154.9 & 4218.89 & 4203 & 4201 & 4207.25 & 20030618 \\
\hline High Core & 434241121311600 & 434240.7 & 1213119.8 & 4840870.3 & 619056.9 & - & - & - & - & - \\
\hline High 1 & 434241121311601 & 434240.6 & 1213119.8 & 4840867.7 & 619058.5 & 4238.72 & 4220 & 4218 & 4222.51 & 20030610 \\
\hline High 2 & 434241121311602 & 434240.6 & 1213119.8 & 4840867.7 & 619058.5 & 4238.72 & 4222 & 4219 & 4222.51 & 20030610 \\
\hline High 3 & 434241121311603 & 434240.6 & 1213119.6 & 4840868.6 & 619062.1 & 4238.45 & 4216 & 4215 & 4222.49 & 20030611 \\
\hline High 4 & 434241121311604 & 434240.6 & 1213119.6 & 4840868.6 & 619062.1 & 4238.45 & 4219 & 4216 & 4222.47 & 20030611 \\
\hline High 5 & 434241121311605 & 434240.6 & 1213119.6 & 4840868.6 & 619062.1 & 4238.45 & 4221 & 4218 & 4222.47 & 20030611 \\
\hline High 6 & 434241121311606 & 434240.6 & 1213119.4 & 4840867.8 & 619065.9 & 4238.45 & 4223 & 4220 & 4222.47 & 20030612 \\
\hline High 7 & 434241121311607 & 434240.6 & 1213119.4 & 4840867.8 & 619073.3 & 4238.56 & 4223 & 4221 & 4222.42 & 20030612 \\
\hline High 8 & 434241121311608 & 434240.6 & 1213115.2 & 4840870.8 & 619160.3 & 4235.22 & 4214 & 4212 & 4222.23 & 20030613 \\
\hline High 9 & 434241121311609 & 434240.6 & 1213115.2 & 4840870.8 & 619160.3 & 4235.22 & 4221 & 4218 & 4222.20 & 20030613 \\
\hline Pine Core & 434210121313400 & 434210.0 & 1213134.1 & 4839918.9 & 618754.5 & - & - & - & - & - \\
\hline Pine 1 & 434210121313401 & 434210.0 & 1213134.1 & 4839917.3 & 618754.5 & 4237.73 & 4223 & 4221 & 4222.34 & 20030609 \\
\hline Pine 2 & 434210121313402 & 434210.0 & 1213134.1 & 4839917.3 & 618754.5 & 4237.73 & 4220 & 4219 & 4222.39 & 20030609 \\
\hline Pine 3 & 434210121313403 & 434210.0 & 1213134.0 & 4839917.5 & 618758.0 & 4237.76 & 4220 & 4219 & 4222.34 & 20030609 \\
\hline
\end{tabular}


Table 3. Well construction information for transect wells, La Pine, Oregon-Continued

[Elevation of static water level at Senior 10 and Senior 11, which are at the western edge of the gravel parking area, may be slightly elevated due to focused recharge from snow plowed from winter storms and piled at the western edge of the parking area; High 8 sampled 6/12/2003, but water level was slow to recover and hence measured 6/13/2003; Date as year, month, day (YYYYMMDD); - , no data]

\begin{tabular}{|c|c|c|c|c|c|c|c|c|c|c|}
\hline Site name & Station number & $\begin{array}{l}\text { Latitude } \\
\text { (NAD83) }\end{array}$ & $\begin{array}{c}\text { Longitude } \\
\text { (NAD83) }\end{array}$ & $\begin{array}{c}\text { UTM North } \\
\text { (NAD83) }\end{array}$ & $\begin{array}{c}\text { UTM East } \\
\text { (NAD83) }\end{array}$ & $\begin{array}{c}\text { Elevation of } \\
\text { land surface } \\
\text { (feet) }\end{array}$ & $\begin{array}{c}\text { Elevation } \\
\text { of top of } \\
\text { screened } \\
\text { interval (feet) }\end{array}$ & $\begin{array}{c}\begin{array}{c}\text { Elevation } \\
\text { of bottom } \\
\text { of screened } \\
\text { interval } \\
\text { (feet) }\end{array} \\
\end{array}$ & $\begin{array}{c}\text { Elevation of } \\
\text { static water } \\
\text { level } \\
\text { (feet) }\end{array}$ & $\begin{array}{c}\text { Date of static water } \\
\text { level measurement } \\
\text { (year/month/day) }\end{array}$ \\
\hline Pine 4 & 434210121313404 & 434210.0 & 1213134.0 & 4839917.5 & 618758.0 & 4237.81 & 4222 & 4221 & 4222.34 & 20030609 \\
\hline Pine 5 & 434210121313405 & 434210.0 & 1213133.5 & 4839917.4 & 618768.3 & 4237.01 & 4217 & 4214 & 4222.19 & 20030616 \\
\hline Pine 6 & 434210121313406 & 434210.0 & 1213133.5 & 4839917.4 & 618768.3 & 4237.01 & 4220 & 4217 & 4222.21 & 20030616 \\
\hline Pine 7 & 434210121313407 & 434209.9 & 1213133.3 & 4839917.0 & 618773.7 & 4237.53 & 4217 & 4215 & 4222.24 & 20030617 \\
\hline Pine 8 & 434210121313408 & 434209.9 & 1213133.3 & 4839917.0 & 618773.7 & 4237.53 & 4221 & 4218 & 4222.25 & 20030617 \\
\hline Pine 9 & 434210121313409 & 434209.9 & 1213133.3 & 4839917.0 & 618773.7 & 4237.53 & 4223 & 4220 & 4222.26 & 20030617 \\
\hline Pine 10 & 434210121313410 & 434210.1 & 1213134.1 & 4839920.4 & 618754.5 & 4237.41 & 4219 & 4216 & 4222.28 & 20030619 \\
\hline Pine 11 & 434210121313411 & 434210.1 & 1213134.1 & 4839920.4 & 618754.5 & 4237.41 & 4223 & 4219 & 4222.27 & 20030619 \\
\hline
\end{tabular}


Table 4. Project samples analyzed for organic wastewater compounds, pharmaceuticals, and coliphage, La Pine, Oregon.

[Standard/Press, Standard and Pressure; DFMW, Drainfield Monitoring Well; FEB, Field Equipment Blank]

\begin{tabular}{|c|c|c|c|c|c|c|}
\hline Type of system & Sample location & Sample type & $\begin{array}{l}\text { Number of } \\
\text { organic } \\
\text { waste- } \\
\text { water } \\
\text { compound } \\
\text { samples } \\
\end{array}$ & $\begin{array}{l}\text { Number of } \\
\text { pharmaceutical } \\
\text { samples }\end{array}$ & $\begin{array}{l}\text { Number of } \\
\text { coliphage } \\
\text { samples; } \\
\text { quantitative } \\
\text { method }\end{array}$ & $\begin{array}{l}\text { Number of coliphage } \\
\text { samples; enrichment, } \\
\text { presence/absence } \\
\text { method }\end{array}$ \\
\hline \multicolumn{7}{|c|}{ Innovative Systems Observation Network } \\
\hline Standard/Press & Septic Tank & Environmental & 4 & 0 & 8 & 0 \\
\hline Sand Filter & Septic Tank & Environmental & 2 & 0 & $3^{\mathrm{a}}$ & 0 \\
\hline Innovative & Septic Tank & Environmental & 14 & 0 & $41^{\mathrm{a}}$ & 0 \\
\hline Sand Filter & Sand Filter Effluent & Environmental & 0 & 0 & $3^{\mathrm{a}}$ & 0 \\
\hline Innovative & End of Pipe & Environmental & 0 & 0 & $45^{\mathrm{a}}$ & 0 \\
\hline Standard/Press & Lysimeter & Environmental & 4 & 0 & 8 & 0 \\
\hline Innovative & Lysimeter & Environmental & 1 & 0 & 2 & 0 \\
\hline Standard/Press & DFMW & Environmental & 4 & 0 & 8 & 0 \\
\hline Sand Filter & DFMW & Environmental & 2 & 0 & $3^{\mathrm{a}}$ & 0 \\
\hline Innovative & DFMW & Environmental & 14 & 0 & $45^{\mathrm{a}}$ & 0 \\
\hline Innovative & DFMW & Resample & 0 & 0 & $4^{b}$ & $4^{\mathrm{b}}$ \\
\hline Innovative & Septic Tank & FEB & 1 & 0 & 1 & 0 \\
\hline Standard/Press & DFMW & FEB & 1 & 0 & 1 & 0 \\
\hline Innovative & Septic Tank & Matrix Spike & 1 & 0 & 1 & 0 \\
\hline Innovative & Septic Tank & Replicate & 2 & 0 & 8 & 0 \\
\hline Standard/Press & Lysimeter & Replicate & 0 & 0 & 6 & 0 \\
\hline Innovative & Lysimeter & Replicate & 0 & 0 & 2 & 0 \\
\hline \multicolumn{7}{|c|}{ Transects Observation Network } \\
\hline Standard/Press & Septic Tank & Environmental & 1 & 1 & 1 & 0 \\
\hline Standard/Press & Transect Well & Environmental & 31 & 11 & 31 & 23 \\
\hline \multirow[t]{2}{*}{ Standard/Press } & Transect Well & Redrill and & & & & \\
\hline & & Resample & 0 & 0 & 3 & 3 \\
\hline Standard/Press & Transect Well & Matrix Spike & 0 & 1 & 0 & 0 \\
\hline Standard/Press & Transect Well & Replicate & 2 & 2 & 2 & 0 \\
\hline
\end{tabular}

a One of the sand filter sites (434741121273401) was not in use for at least a few weeks during fall 2003, and thus was not sampled during the fall 2003 sampling synoptic. Resources that had been targeted for that sand filter site were instead used to collect an additional set of samples from one of the innovative sites (a FAST site; 434952121290602). Thus, one of the sand filter sites was sampled only once (spring 2003), whereas one of the innovative system sites was sampled three times (once in spring 2003, and twice in fall 2003).

${ }^{\mathrm{b}}$ These samples were collected to evaluate detections reported for earlier DFMW samples; the repeat samples were analyzed only for the coliphage that were detected in the earlier DFMW samples (i.e. for any given sample, either F-specific or somatic coliphage, but not both). 


\section{Field Methods}

\section{Innovative Systems Network}

The onsite wastewater treatment systems sampled for this study had already been installed as part of ODEQ/DCEHD monitoring efforts. No effort was made to install sterile monitoring ports in the onsite wastewater treatment systems themselves. The lysimeters, sand filter samplers, and downgradient monitoring wells were not sterilized prior to installation, but any contamination (microbial or chemical) present on the surfaces of the lysimeters, sand filter samplers, or well materials upon installation was flushed over the periods of months to (usually) years of monitoring (with sterile sampling equipment) before the sampling reported here. Lysimeters were simple PVC (polyvinyl chloride) half-pipes that emptied into stainless steel collection buckets. Lysimeters were installed in native sediment, 1 foot below the bottoms of the 2-foot-deep trenches that house the onsite wastewater treatment system drainfield lines. Sand filters were instrumented in the same manner as lysimeters (i.e., with half-pipes and stainless steel collection buckets), but with the half-pipes located at the base of the sand filter material (i.e., installed at the sand filter/native soil interface during sand filter construction). Drainfield monitoring wells were installed using two methods: hollow stem auger and 2-inch-diameter direct push. Drilling fluids were not used with either well-installation method. Precleaned, plastic-wrapped 0.375-inch-inside-diameter, 0.50-inch-outside-diameter PVC screens and casing were used. Wells were sand-packed to 1 foot above screens and sealed with bentonite to land surface. Drainfield monitoring wells were developed by pumping with a peristaltic pump until ground water was visibly clear.

Samples from septic tanks and end-of-pipe collection chambers (following innovative onsite treatment) were collected in sterile (autoclaved) glass bottles that had been precleaned by standard USGS cleaning procedures for collection of organic compounds (solution of non-phosphate-based detergent, tapwater rinses, deionized-water rinses, methanol rinse; U.S. Geological Survey, 1999). The bottles were filled either directly by dipping into septic tanks or end-of-pipe collection chambers or by filling (by pouring) from precleaned, plastic-wrapped disposable Teflon bailers dipped into septic tanks or end-of-pipe collection chambers. Direct dipping was done with glass bottles inserted into precleaned and sterilized (dilute sodium hypochlorite solution) polyethylene dippers. Dipping and bailing was done in the centroid of the septic tank or end-of-pipe collection chamber. To minimize the potential for cross-contamination, collection bottles and bailers were used for only one type of sample (septic tank, end-of-pipe, sand filter effluent, lysimeter, or drainfield monitoring well) at a site and then discarded. Samples from lysimeters and sand filters were collected from stainless steel collection buckets that had been precleaned and sterilized (autoclaved) by the same methods as the glass bottles. Samples from drainfield monitoring wells were collected with Teflon tubing (with a 3-foot section of C-flex peristaltic tubing for use at the peristaltic pump head) that had been precleaned (solution of non-phosphate-based detergent, tapwater rinses, deionizedwater rinses, and methanol rinse for the Teflon tubing but not the peristaltic tubing), followed by sterilization with a 0.005 percent sodium hypochlorite solution, neutralization with a 0.005 percent sodium thiosulfate solution, and final deionized water rinses. Ground-water samples were collected following a well purge of at least three bore volumes. Tubing was used once and then discarded.

Bottles for coliphage and nutrient samples were filled onsite by collection of raw (unfiltered) sample water directly from the glass bottles or Teflon bailers used to collect the samples, or from pump tubing. Nutrient samples were preserved with sulfuric acid to $\mathrm{pH}<2$. Coliphage sample bottles were precleaned and sterilized (autoclaved) polypropylene bottles; nutrient sample bottles, precleaned high-density polyethylene bottles. Samples for analysis of organic wastewater compounds and chloride were filtered at the DCEHD Laboratory. Filtering occurred on the same day that the raw samples were collected. Samples for organic wastewater compounds were first filtered through baked $\left(450^{\circ} \mathrm{C}\right) 1.2-\mu \mathrm{m}$ glass fiber filters, then through baked $\left(450^{\circ} \mathrm{C}\right) 0.7-\mu \mathrm{m}$ glass fiber filters, with filters housed in precleaned (solution of non-phosphate-based detergent, tapwater rinses, deionized-water rinses, methanol rinse) glassware. The sample filtrate for organic wastewater compound analysis was poured into baked $\left(450^{\circ} \mathrm{C}\right)$ amber glass bottles. Samples for chloride were filtered through disposable $0.45-\mu \mathrm{m}$ nominal-pore-size capsule filters. The sample filtrate for chloride analysis was collected directly in precleaned (solution of non-phosphate-based detergent, tapwater rinses, and deionized-water rinses) high-density polyethylene bottles. All sample bottles were used once and then discarded.

All samples were kept on ice, in coolers, from the time of collection to arrival for processing at the DCEHD laboratory. Samples remained iced at the DCEHD laboratory except during filtering. Samples were shipped (iced) on the same day they were collected, by overnight courier, to analytical laboratories.

\section{Transects}

Transect wells were temporary, 1-inch-diameter directpush wells. Drilling fluids were not used. Screens were stainless steel, in 2- and 4-foot lengths (although screens were not always fully deployed). Drilling rods were stainless steel; disposable drive points, steel. The disposable steel drive points were washed in the laboratory (solution of non-phosphatebased detergent, tapwater rinses, deionized-water rinses, but no methanol rinse because of the presence of a rubber O-ring) and then sterilized (autoclaved). (These drive-point tips were removed prior to sampling by pushing the point out of the bottom of the screen into the aquifer.) Screens, drilling rods, and the stainless steel rods used to push out the disposable 
steel drive point all were steam cleaned, rinsed with deionized water, and given a final rinse in organic-blank water. Organicblank water was provided by the USGS NWQL after testing to ensure purity. Samples were collected with Teflon tubing (with a 3-foot section of C-flex peristaltic tubing for use at the peristaltic pump head) that had been precleaned (solution of non-phosphate-based detergent, tapwater rinses, deionizedwater rinses, and methanol rinse for the Teflon tubing but not the peristaltic tubing), followed by sterilization with a 0.005 percent sodium hypochlorite solution, neutralization with a 0.005 percent sodium thiosulfate solution, and final deionized water rinses. Tubing was used once and then discarded.

Water samples were collected following a well purge of at least three bore volumes. Sterile (autoclaved) polypropylene bottles for coliphage samples were filled onsite by collection of raw (unfiltered) sample water directly from Teflon tubing. Nitrate and chloride samples were filtered in-line through disposable 0.45 - $\mu$ m nominal-pore-size capsule filters. Samples for organic wastewater compounds and pharmaceuticals were filtered in-line through baked $\left(450^{\circ} \mathrm{C}\right) 0.7-\mu \mathrm{m}$ glass fiber filters, with filters housed in cleaned (solution of non-phosphate-based detergent, tapwater rinses, deionized-water rinses, methanol rinse) aluminum filter holders, directly into baked $\left(450^{\circ} \mathrm{C}\right)$ amber glass bottles. Sample bottles were used once and then discarded. All samples were kept on ice, in coolers, from the time of collection to arrival at analytical laboratories by overnight courier. More detailed description of USGS sample collection and processing protocols can be found in U.S. Geological Survey (1999).

\section{Analytical Methods}

Most innovative system network samples for chloride and nutrients were analyzed by ODEQ in Portland, Oregon. ODEQ analytical techniques were as follows: chloride, automated ferricyanide; nitrate, automated cadmium reduction; ammonium plus organic nitrogen, Kjeldahl digestion and colorimetry (automated phenate); total $\mathrm{N}$ was calculated as the sum of nitrate and Kjeldahl nitrogen. These techniques are described by Clesceri and other (1998). Samples from the innovative system network that were analyzed for coliphage during December 2003 were analyzed for chloride at the USGS NWQL (24 samples, analyzed by ion-exchange chromatography; Fishman, 1993).

Transect ground-water samples were analyzed for field parameters, chloride, nitrate, organic wastewater compounds, pharmaceuticals (Senior transect only), and coliphage. The La Pine Senior Center onsite wastewater treatment system was sampled for organic wastewater compounds, pharmaceuticals, and coliphage. All of these transect samples were analyzed by the USGS. All innovative system network organic wastewater compound samples (as well as the 24 innovative system network chloride samples) also were analyzed by the USGS.

Field parameters-dissolved oxygen, $\mathrm{pH}$, temperature, and specific conductance-were measured electrometrically in situ, but after collection of aqueous samples (to avoid contamination of the sample tubing during measurement of field parameters). Chloride, nitrate, organic wastewater compounds, and pharmaceuticals were analyzed at the USGS NWQL; coliphage were analyzed by the USGS Ohio District Microbiology Laboratory in Columbus, Ohio. Chloride was analyzed by ion-exchange chromatography (Fishman, 1993); nitrate, by automated-segmented flow colorimetry (Fishman, 1993); organic wastewater compounds, by solid-phase extraction and capillary-column gas chromatography/mass spectrometry (GC/ MS) (Zaugg and others, 2002).

Pharmaceuticals were analyzed by solid-phase extraction and elution followed by high-performance liquid chromatography/mass spectrometry (HPLC/MS). This analytical method is still under development, hence, a formal USGS methods document has yet to be published. The method and the laboratory used for analysis of pharmaceutical samples in this report are identical to those used and described by Kolpin and others (2002) in their study of pharmaceutical compounds in surface water, although the suite of pharmaceuticals described in this report differs from the suite reported by Kolpin and others (2002). Of the 21 pharmaceuticals reported by Kolpin and others (2002), 15 are reported here; the other 6 have either been excluded from determination by the method because of low average analyte recovery in routine laboratory spikes of reagent-grade water (spike compounds: digoxin, digoxigenin, enalaprilat, and paroxetine metabolite) or were excluded from determination from the current group of samples because of low analyte recovery in laboratory spikes of reagent-grade water concurrently analyzed with the environmental samples of this study (fluoxetine, with recoveries of 17, 19, 24 and 28 percent, and metformin, with recoveries of 1, 3, 3, and 3 percent). Three pharmaceuticals that were not analyzed by Kolpin and others (2002) have since been included for analysis by the method and concentrations are reported here [thiabendazole, American Chemical Society Chemical Abstracts Service Registry Number (CAS) 148-79-8, an anthelmintic; diphenhydramine, CAS 58-73-1, an antihistamine; and carbamazapine, CAS 298-46-4, an anticonvulsant]. The provisional laboratory reporting levels (LRLs) used here have been revised since the publication of results by Kolpin and others (2002), but remain provisional while method development continues.

The GC/MS (organic wastewater compound) and HPLC/ MS (pharmaceutical) analytical methods have enhanced analyte-identification capabilities (Childress and others, 1999). The identification of a compound is made from chromatographic signal and retention time matching with additional qualifying information-the presence of characteristic mass spectal ions with known ion ratios-provided by the mass spectrometric detector.

All sites were sampled for male-specific $\left(\mathrm{F}^{+}\right)$and somatic coliphage by the single agar layer procedure ("quantitative method," USEPA method 1602; U.S. Environmental Protection Agency, 2001a). The single agar layer method is a plaque assay method. For this method, $100-\mathrm{mL}$ water samples are combined with host bacteria and nutrients, poured into plates, 
and incubated. Viable coliphage present in the water sample will infect bacteria cells and replicate, causing death of bacterial cells in the process. This process continues until a plaque (loss of bacteria in the plate) forms. Thus, each plaque represents one viable coliphage particle. Results from the quantitative method are reported as PFU (plaque forming units)/100 $\mathrm{mL}$. At some sites, samples were collected for analysis by two methods: the quantitative method, and a two-step enrichment procedure ("enrichment, presence/absence," USEPA method 1601; U.S. Environmental Protection Agency, 2001b). For this method, 1-liter water samples are combined with host bacteria and nutrients, and incubated for 24 hours at $35^{\circ} \mathrm{C}$. This growth (enrichment) step increases the number of coliphage. Subsamples then are poured onto a bacterial lawn and incubated overnight. Coliphage presence is indicated by bacterial lysis.

Sediment samples from split spoon cores at transect sites were analyzed for particle size and organic carbon concentration. Particle-size analysis was done by wet sieving (Guy, 1969) at the USGS Cascade Volcano Observatory in Vancouver, Washington. Particles with nominal diameters of less than 0.062 millimeters ( 0.0024 inches) are operationally considered smaller than sand-sized particles (i.e., are considered clay and silt). Organic carbon concentration was determined by the Mineral Resources Team, USGS, in Denver, Colorado. Total carbon was measured by combustion to generate carbon dioxide, followed by measurement with a solid state infrared detector (Taggart, 2002). Carbonate carbon was measured by coulometric titration (Taggart, 2002). Organic carbon was determined by difference.

\section{Reporting of Data}

When organic wastewater compounds are not detected during sample analysis, concentrations are censored at (reported as less than) the laboratory reporting level (LRL). Censored data are common in hydrologic applications (Helsel and Hirsch, 1992); a censored concentration represents a concentration below the censoring level, and may be zero or greater than zero. The small sample volumes that were available for some onsite wastewater samples (due to filter clogging) and lysimeter samples (due to low lysimeter production) lead to decreased analytical sensitivity, and necessitated raised LRLs for some analytes in those samples. When organic wastewater compounds are detected and quantifiable at concentrations below the LRL, but above the published (Zaugg and others, 2002) method detection limit (MDL), the analytical results are reported with a remark code ("E", for estimated concentration). (An "E" code is also used in other instances where quantification [but not identification] is less certain than prescribed by analytical protocols for "typical" operational conditions; this uncertainty in concentration can occur in any analysis, not just analysis for organic wastewater compounds. As examples, an "E" code is also used when analyte detection is at a concentration that is less than the concentration of the lowest calibration standard or greater than the highest calibration standard, or if the analyte exhibits longterm variable or poor recovery in routine laboratory spikes of reagent-grade water, or if matrix problems are present.) Occasionally, organic wastewater compounds are detected at concentrations below the published MDL of Zaugg and others (2002). This circumstance occurs because the actual low-level method sensitivity tends to vary from day to day relative to long-term average sensitivity. In such instances, when organic wastewater compounds are detected at concentrations below the published MDL, results are represented by a remark code ("M", presence is verified, but concentration is not quantified). Many organic wastewater compound detections in this study were at low-level concentrations greater than the published MDL and less than the LRL ("E" coded detections). Concentrations of pharmaceuticals, however, are uniformly censored at their provisional LRLs (concentrations below provisional LRLs were not reported) because the pharmaceutical analytical method is still under development and MDLs based upon long-term data have not been established. Finally, analytical data in this report may be censored at a concentration greater than the LRL if project quality-control data indicate the need. Such a censoring level is called a project censoring level. For example, contamination in field equipment blanks may indicate the presence of field and (or) laboratory processing or analytical contamination that may result in adoption of a project censoring level greater than the LRL.

\section{Results and Discussion}

\section{Transect Sediment Cores}

One continuous sediment core was collected from a random location at each of the three transects. Lithologic descriptions, particle sizes, and organic carbon contents of sediment from these cores are given in table 5. The sediment is predominantly sand with only minor amounts of clay, consistent with lithology reported in drillers' well logs from the vicinity of the transects. The sediment tends to be low in organic carbon (generally $<0.2$ percent organic carbon). Although one core provides only a general indication of subsurface geology at any given site, these data do provide information about the overall lithologic framework for wastewater analyte occurrence in this study, and may provide a basis for possible future comparative studies.

\section{Organic Wastewater Compounds}

Concentrations of organic wastewater compounds from the innovative system network (20 onsite wastewater treatment systems, 5 downgradient lysimeters, and 20 drainfield monitoring wells) are given in table 6. Concentrations of organic 
["Percent finer than sand" is defined to be composed of particles with nominal diameters of less than 0.062 millimeters or 0.0024 inches; —, no data]

\begin{tabular}{|c|c|c|c|c|}
\hline $\begin{array}{l}\text { Top of } \\
\text { interval } \\
\text { (feet below } \\
\text { land surface) }\end{array}$ & $\begin{array}{c}\text { Bottom of interval } \\
\text { (feet below land } \\
\text { surface) }\end{array}$ & $\begin{array}{l}\text { Percent } \\
\text { organic } \\
\text { carbon } \\
\end{array}$ & $\begin{array}{c}\text { Percent finer } \\
\text { than sand }\end{array}$ & Lithologic Description \\
\hline \multicolumn{5}{|c|}{ Senior Transect } \\
\hline 0.0 & 5.5 & - & - & Weathered pumice in a matrix of fine to medium sand \\
\hline 5.5 & 8.5 & $<0.05$ & 7.7 & Fine to medium sand \\
\hline 8.5 & 9.3 & - & - & Fine to medium sand with pebble clasts \\
\hline 9.3 & 11.0 & 0.07 & 35.5 & Silt with sparse fine pebble clasts, and with zones of iron oxides \\
\hline 11.0 & 13.4 & 0.06 & 5.0 & Medium sand with interbeds of silty clay \\
\hline 13.4 & 17.8 & 0.05 & 3.2 & Very coarse sand to fine gravel, with zones of iron oxides \\
\hline 17.8 & 23.0 & $<0.05$ & 13.5 & Very fine to fine sand with interbeds of silty clay \\
\hline \multicolumn{5}{|r|}{ High Transect } \\
\hline 0.0 & 3.7 & 0.11 & 12.7 & Weathered pumice clasts, fine to coarse \\
\hline 3.7 & 8.5 & 0.06 & 11.2 & Fine to coarse sand, some fine gravel \\
\hline 8.5 & 12.0 & 0.10 & 63.0 & Silt to very fine sand \\
\hline 12.0 & 16.0 & $<0.05$ & 18.7 & Medium sand to fine pebbles \\
\hline 16.0 & 20.0 & 0.07 & 13.9 & Very fine to fine sand, with interbedded clayey ash \\
\hline 20.0 & 21.0 & 0.07 & 15.9 & Very fine to fine sand \\
\hline 21.0 & 23.0 & 0.15 & 62.7 & Silt to very fine sand \\
\hline 23.0 & 24.0 & 0.19 & 33.3 & Very fine to fine sand \\
\hline \multicolumn{5}{|r|}{ Pine Transect } \\
\hline 0.0 & 1.0 & 0.63 & 38.0 & Very fine pumice pebbles, moderately weathered, with roots and organic debris \\
\hline 1.0 & 10.2 & 0.07 & 23.2 & $\begin{array}{l}\text { Very fine to medium sand with very fine pumice pebbles; some sand of basalt/cinder; } \\
\text { silt loam and sandy loam interbeds }\end{array}$ \\
\hline 10.2 & 14.0 & 0.06 & 5.5 & Coarse sand to fine pebbles, mainly of basalt/cinder. \\
\hline 14.0 & 14.3 & 0.06 & 20.0 & Very fine to fine loamy sand, with basalt/cinder clasts and liberated plagioclase \\
\hline 14.3 & 17.3 & $<0.05$ & 4.8 & Medium to very coarse sand \\
\hline 17.3 & 18.1 & 0.06 & 25.2 & Fine loamy sand \\
\hline 18.1 & 19.0 & 0.06 & 3.5 & Medium to very fine pebbles, with sparse liberated pyroxene and plagioclase crystals \\
\hline
\end{tabular}


wastewater compounds from the transects (31 monitoring wells installed along three transects, along with a sample from 1 of the 3 onsite wastewater treatment systems for these transects) are given in table 7 .

Quality-control data associated with these environmental data are presented in Appendix B. The presence of phenol at a concentration of E $0.4 \mathrm{~g} / \mathrm{L}$ in one field equipment blank (Appendix B) indicates the potential for a positive bias in phenol concentrations. The approach taken in this study to account for this potential bias was to censor environmental phenol concentrations (tables 5 and 6) at a project censoring level of $4 \mathrm{~g} / \mathrm{L}, 10$ times the concentration detected in the one field equipment blank. Censoring at 10 times the blank contamination concentration represents one approach that has been used in compliance monitoring (U.S. Environmental Protection Agency, 1993). A project censoring level 10 times the concentration detected in the one field equipment blank is sufficiently high to avoid complicating effects of possible sampling or analytical contamination yet still allow uncensored retention of phenol concentrations greater than $4 \mathrm{~g} / \mathrm{L}$. Two other organic wastewater compounds were detected at low (nonquantifiable) concentrations ("M" coded data): 1,4-dichlorobenzene and p-cresol. The presence of such low-level contamination in field equipment blanks suggests that " $\mathrm{M}$ " coded concentration results in environmental samples in general should be viewed with caution. In this report, "M" coded environmental data will be considered for discussion purposes equivalent to nondetects at the LRL. All other aspects of the quality-control data were satisfactory.

In addition to the quality assurance provided by the quality-control data, some of the environmental data can be used for quality-assurance purposes. Environmental samples that are low in chloride are less likely to have a component of onsite wastewater than are samples that are high in chloride. In this manner, environmental samples that are low in chloride may represent what can be called environmental blanks. The use of low-chloride samples to represent environmental blanks rests upon assumptions that the so-called low concentration of chloride represents chloride only from sources other than onsite wastewater, and that the only source of organic wastewater compounds to ground water is onsite wastewater. Regardless of the concentration of chloride that is considered to represent ground water unimpacted by onsite wastewater, it is always possible that a low-chloride sample may contain a small component of onsite wastewater, and organic wastewater compounds can enter aquifers from many diffuse sources in addition to onsite wastewater (e.g., plasticizers from urban infrastructure, gasoline components from roadways, cleaning agents from washing of automobiles, etc.). Thus, analysis of low-chloride ground water is not as robust a measure of sampling and analytical contamination as is analysis of field equipment blanks. Nevertheless, the approach of using environmental data to characterize potential bias can be useful when evaluated in the context of these limitations.
There are few sources of chloride loading to La Pine ground water. There is a near-absence of agriculture (see Introduction), and evaporites do not occur in the volcanic rocks and sediment of the La Pine area. The State Department of Transportation and the Deschutes County Road Department apply magnesium chloride to reduce ice buildup, and the Deschutes County Road Department applies it for dust control, but use is very limited (Patrick Creedican, Oregon Department of Transportation, written commun., 2005, and Dee Martin, Deschutes County Road Department, oral commun., 2005). Magnesium chloride is used to control ice at selected intersections, grades, and curves on paved roads (mostly on or near Highways 97 and 31; figure 1) (Patrick Creedican, Oregon Department of Transportation, written commun., 2005, and Dee Martin, Deschutes County Road Department, oral commun., 2005). Magnesium chloride is used for dust control on two 1-mile-long sections of unpaved roads in the Deschutes County portion of the study area (Roger Olson, Deschutes County Road Department, written commun., 2005). Atmospheric deposition and onsite wastewater are the dominant sources of chloride to study-area ground water. Atmospheric precipitation in the La Pine area contains low concentrations of chloride, although the magnitude of the chloride flux from precipitation may be significant as a result of the widespread distribution of this chloride source. The National Atmospheric Deposition Program precipitation-weighted mean chloride concentration for the Silver Lake Ranger Station site (44 miles southeast of La Pine) for calendar year 2002 was $0.08 \mathrm{mg} / \mathrm{L}$ (National Atmospheric Deposition Program, no date). Chloride concentrations in onsite wastewater are high. The median chloride concentration for the 20 innovative network onsite wastewater samples was $43 \mathrm{mg} / \mathrm{L}$ (table 6) (a 21st sample of onsite wastewater, associated with the transects, was not analyzed for chloride). If we arbitrarily assume that a chloride concentration in ground water of less than $2 \mathrm{mg} / \mathrm{L}$ represents ground water that does not contain a significant component of onsite wastewater, then 5 of the 31 transect sites can be treated as environmental blanks (Senior 7, 9, 11, and High Lakes 8, 9; table 7). (Concentrations of nitrate in these five samples were as high as $0.72 \mathrm{mg} \mathrm{N} / \mathrm{L}$, and could represent a degree of influence of onsite wastewater despite the low chloride concentrations.) No organic wastewater compounds were detected in any of these samples. One additional environmental blank is available from the drainfield monitoring wells from the innovative system network (the AdvanTex drainfield monitoring well, with chloride at $0.6 \mathrm{mg} / \mathrm{L}$; table 6 ). (The concentration of nitrate in this sample, $0.002 \mathrm{mg} \mathrm{N} / \mathrm{L}$, may be low due to denitrification, as indicated by the low dissolved oxygen concentration of $0.1 \mathrm{mg} / \mathrm{L}$ for this sample). 
Table 6. Concentrations of organic wastewater compounds in water from traditional and innovative onsite wastewater treatment systems, downgradient lysimeters, and downgradient ground water, La Pine, Oregon, 2003.

[Benzophenone concentrations are reported in this table as they were reported by the laboratory, but are interpreted in the report relative to a project censoring level of 1 microgram per liter; see discussion of organic wastewater compounds in the results and discussion section of the text for explanation of this project censoring level; Type of onsite system, see descriptions in table 1; Sample Type: STE, septic tank effluent, LYS, lysimeter, DFMW, drainfield monitoring well; Date as year, month, day (YYYYMMDD); Time in hours and minutes, military; Chloride in milligrams per liter, from Oregon Department of Environmental Quality; Nitrite-plus-nitrate, and Total N, in milligrams N per liter, from Oregon Department of Environmental Quality; organic wastewater compounds in micrograms per liter; surrogate recoveries in percent; parameter codes for organic wastewater compound names: Pxxxx; "E", estimated; "<", less than; "M", presence verified, not quantified; Sample 434437121295301: organic wastewater compound sample collected 20030507, and chloride and nitrite-plus-nitrate samples collected 20030505; —, not reported (see text)]

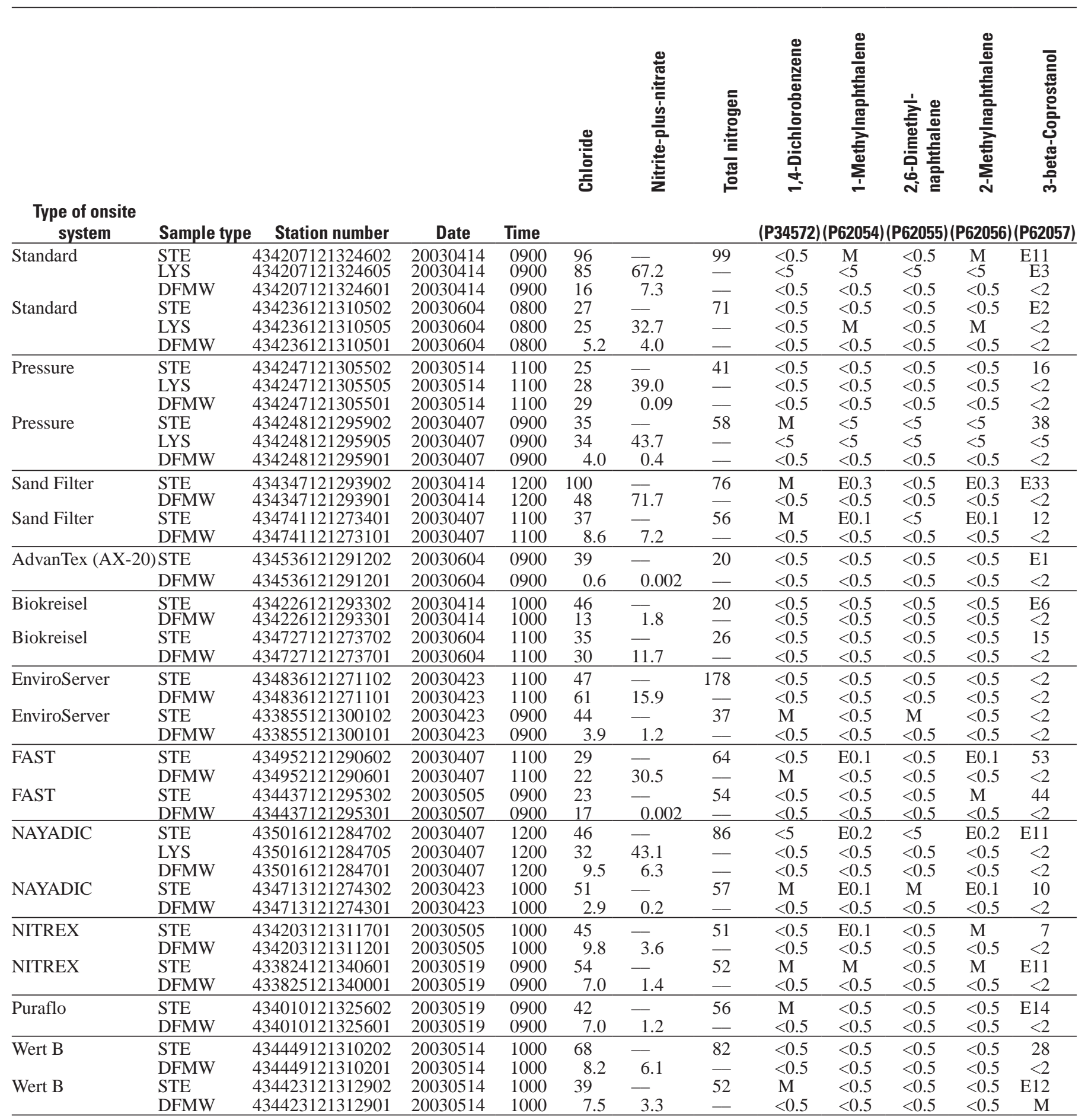


Table 6. Concentrations of organic wastewater compounds in water from traditional and innovative onsite wastewater treatment systems, downgradient lysimeters, and downgradient ground water, La Pine, Oregon, 2003. - Continued

[Benzophenone concentrations are reported in this table as they were reported by the laboratory, but are interpreted in the report relative to a project censoring level of 1 microgram per liter; see discussion of organic wastewater compounds in the results and discussion section of the text for explanation of this project censoring level; Type of onsite system, see descriptions in table 1; Sample Type: STE, septic tank effluent, LYS, lysimeter, DFMW, drainfield monitoring well; Date as year, month, day (YYYYMMDD); Time in hours and minutes, military; Chloride in milligrams per liter, from Oregon Department of Environmental Quality; Nitrite-plus-nitrate, and Total N, in milligrams N per liter, from Oregon Department of Environmental Quality; organic wastewater compounds in micrograms per liter; surrogate recoveries in percent; parameter codes for organic wastewater compound names: Pxxxx; "E", estimated; "〈", less than; "M", presence verified, not quantified; Sample 434437121295301: organic wastewater compound sample collected 20030507, and chloride and nitrite-plus-nitrate samples collected 20030505; —, not reported (see text)]

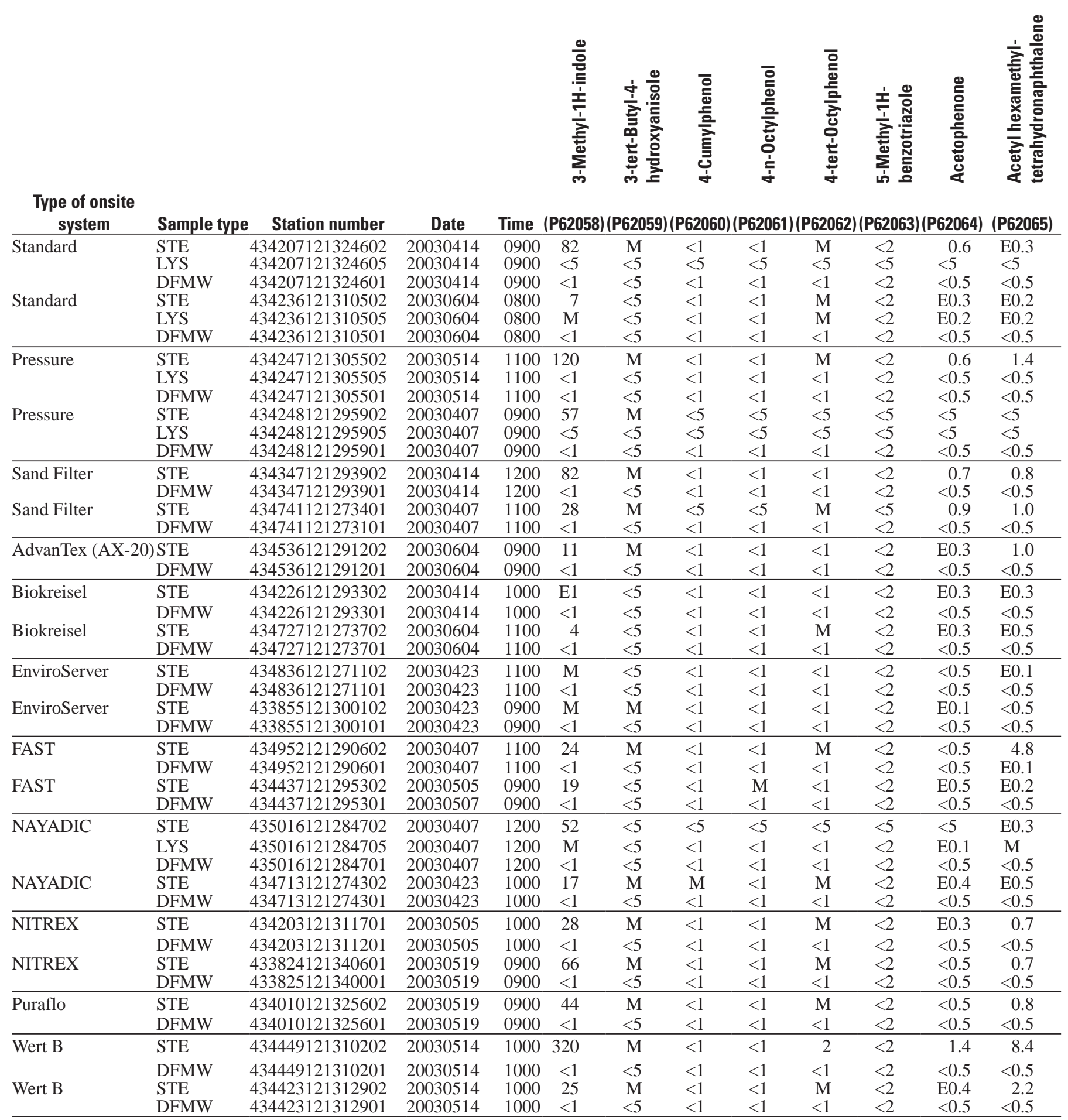


Table 6. Concentrations of organic wastewater compounds in water from traditional and innovative onsite wastewater treatment systems, downgradient lysimeters, and downgradient ground water, La Pine, Oregon, 2003.—Continued

[Benzophenone concentrations are reported in this table as they were reported by the laboratory, but are interpreted in the report relative to a project censoring level of 1 microgram per liter; see discussion of organic wastewater compounds in the results and discussion section of the text for explanation of this project censoring level; Type of onsite system, see descriptions in table 1; Sample Type: STE, septic tank effluent, LYS, lysimeter, DFMW, drainfield monitoring well; Date as year, month, day (YYYYMMDD); Time in hours and minutes, military; Chloride in milligrams per liter, from Oregon Department of Environmental Quality; Nitrite-plus-nitrate, and Total N, in milligrams N per liter, from Oregon Department of Environmental Quality; organic wastewater compounds in micrograms per liter; surrogate recoveries in percent; parameter codes for organic wastewater compound names: Pxxxx; "E", estimated; “<”, less than; "M", presence verified, not quantified; Sample 434437121295301: organic wastewater compound sample collected 20030507, and chloride and nitrite-plus-nitrate samples collected 20030505; —, not reported (see text)]

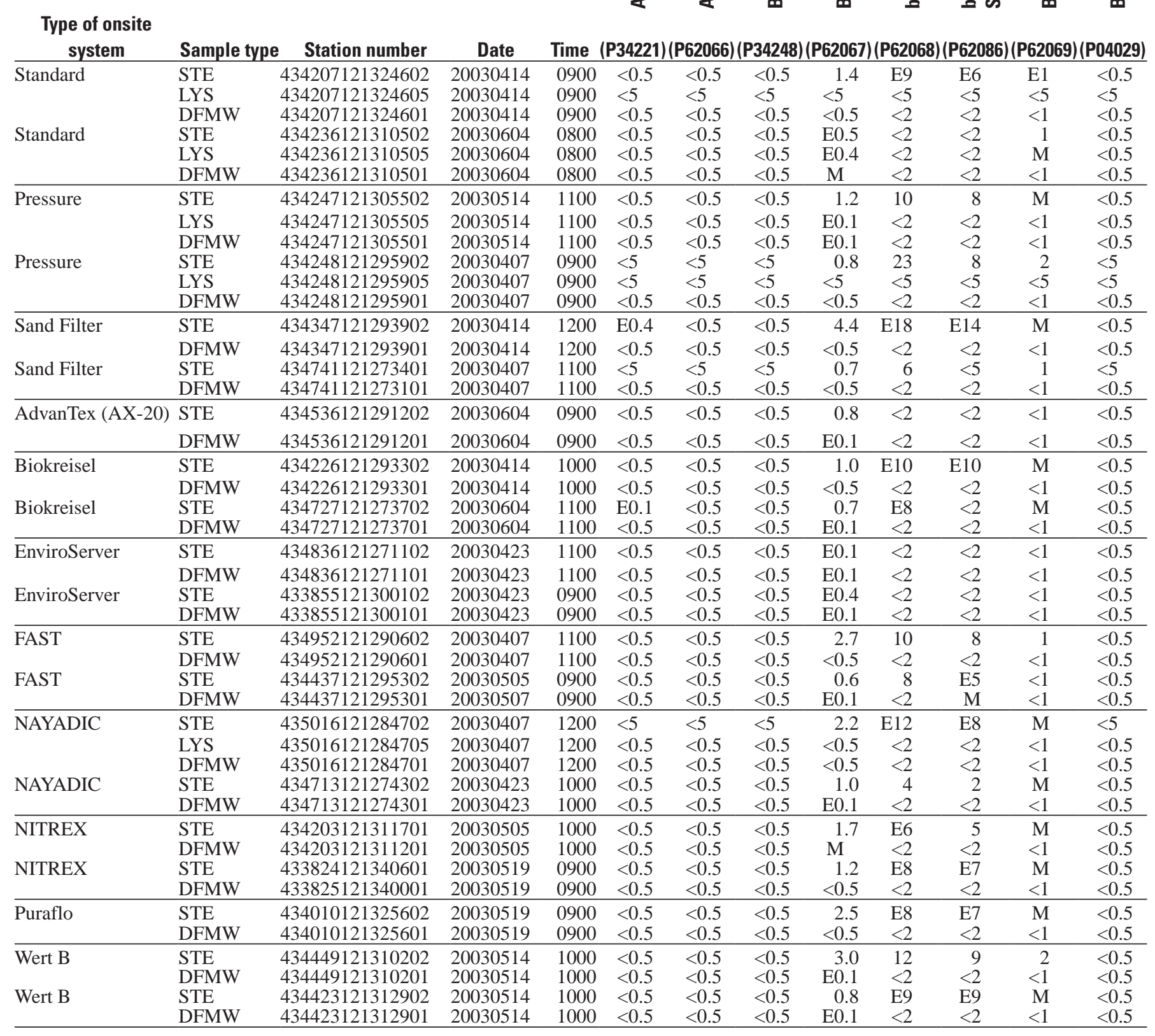


Table 6. Concentrations of organic wastewater compounds in water from traditional and innovative onsite wastewater treatment systems, downgradient lysimeters, and downgradient ground water, La Pine, Oregon, 2003.-Continued

[Benzophenone concentrations are reported in this table as they were reported by the laboratory, but are interpreted in the report relative to a project censoring level of 1 microgram per liter; see discussion of organic wastewater compounds in the results and discussion section of the text for explanation of this project censoring level; Type of onsite system, see descriptions in table 1; Sample Type: STE, septic tank effluent, LYS, lysimeter, DFMW, drainfield monitoring well; Date as year, month, day (YYYYMMDD); Time in hours and minutes, military; Chloride in milligrams per liter, from Oregon Department of Environmental Quality; Nitrite-plus-nitrate, and Total N, in milligrams N per liter, from Oregon Department of Environmental Quality; organic wastewater compounds in micrograms per liter; surrogate recoveries in percent; parameter codes for organic wastewater compound names: Pxxxx; "E", estimated; "<", less than; "M", presence verified, not quantified; Sample 434437121295301: organic wastewater compound sample collected 20030507, and chloride and nitrite-plus-nitrate samples collected 20030505; —, not reported (see text)]

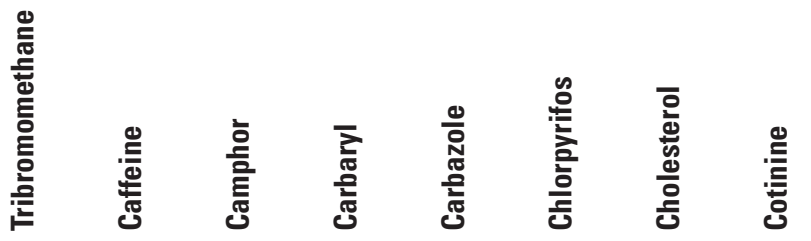

\begin{tabular}{|c|c|c|c|c|c|c|c|c|c|c|c|c|}
\hline $\begin{array}{c}\text { Iype or onsite } \\
\text { system }\end{array}$ & Sample type & Station number & Date & Time & (P34288) & (P50305) & (P62070) & (P82680) & (P62071) & (P38933) & (P62072)( & (P62005) \\
\hline \multirow[t]{3}{*}{ Standard } & STE & 434207121324602 & 20030414 & 0900 & $<0.5$ & 140 & 3.0 & $<1$ & $<0.5$ & $<0.5$ & E33 & $<1$ \\
\hline & LYS & 434207121324605 & 20030414 & 0900 & $<5$ & E0.2 & $<5$ & $<5$ & $<5$ & $<5$ & E5 & $<5$ \\
\hline & DFMW & 434207121324601 & 20030414 & 0900 & $<0.5$ & $<0.5$ & $<0.5$ & $<1$ & $<0.5$ & $<0.5$ & $<2$ & $<1$ \\
\hline \multirow{3}{*}{ Standard } & STE & 434236121310502 & 20030604 & 0800 & $<0.5$ & 2.2 & E0.1 & $<1$ & $<0.5$ & $<0.5$ & E3 & $<1$ \\
\hline & LYS & 434236121310505 & 20030604 & 0800 & $<0.5$ & E0.3 & $<0.5$ & $<1$ & $<0.5$ & $<0.5$ & $<2$ & $<1$ \\
\hline & DFMW & 434236121310501 & 20030604 & 0800 & $<0.5$ & $<0.5$ & $<0.5$ & $<1$ & $<0.5$ & $<0.5$ & $<2$ & $<1$ \\
\hline \multirow{3}{*}{ Pressure } & STE & 434247121305502 & 20030514 & 1100 & $<0.5$ & 5.1 & 0.9 & $<1$ & $<0.5$ & $<0.5$ & 33 & 2 \\
\hline & LYS & 434247121305505 & 20030514 & 1100 & $<0.5$ & $<0.5$ & $<0.5$ & $<1$ & $<0.5$ & $<0.5$ & $<2$ & $<1$ \\
\hline & DFMW & 434247121305501 & 20030514 & 1100 & $<0.5$ & $<0.5$ & $<0.5$ & $<1$ & $<0.5$ & $<0.5$ & $<2$ & $<1$ \\
\hline \multirow[t]{3}{*}{ Pressure } & STE & 434248121295902 & 20030407 & 0900 & $<5$ & 90 & E0.5 & $<5$ & $<5$ & $<5$ & 110 & $<5$ \\
\hline & LYS & 434248121295905 & 20030407 & 0900 & $<5$ & M & $<5$ & $<5$ & $<5$ & $<5$ & E2 & $<5$ \\
\hline & DFMW & 434248121295901 & 20030407 & 0900 & $<0.5$ & $<0.5$ & $<0.5$ & $<1$ & $<0.5$ & $<0.5$ & $<2$ & $<1$ \\
\hline \multirow[t]{2}{*}{ Sand Filter } & STE & 434347121293902 & 20030414 & 1200 & $<0.5$ & 99 & 1.1 & $<1$ & $<0.5$ & $<0.5$ & E52 & $<1$ \\
\hline & DFMW & 434347121293901 & 20030414 & 1200 & $<0.5$ & $<0.5$ & $<0.5$ & $<1$ & $<0.5$ & $<0.5$ & $<2$ & $<1$ \\
\hline \multirow[t]{2}{*}{ Sand Filter } & STE & 434741121273401 & 20030407 & 1100 & $<5$ & 8.8 & 1.0 & $<5$ & $<5$ & $<5$ & 24 & $<5$ \\
\hline & DFMW & 434741121273101 & 20030407 & 1100 & $<0.5$ & $<0.5$ & $<0.5$ & $<1$ & $<0.5$ & $<0.5$ & $<2$ & $<1$ \\
\hline \multicolumn{2}{|c|}{ Advantex (AX-20)STE } & 434536121291202 & 20030604 & 0900 & $<0.5$ & 3.8 & 3.4 & $<1$ & $<0.5$ & $<0.5$ & E2 & $<1$ \\
\hline & DFMW & 434536121291201 & 20030604 & 0900 & $<0.5$ & $<0.5$ & $<0.5$ & $<1$ & $<0.5$ & $<0.5$ & $<2$ & $<1$ \\
\hline \multirow[t]{2}{*}{ Biokreisel } & STE & 434226121293302 & 20030414 & 1000 & $<0.5$ & 9.2 & E0.2 & $<1$ & $<0.5$ & $<0.5$ & E20 & $<1$ \\
\hline & DFMW & 434226121293301 & 20030414 & 1000 & $<0.5$ & $<0.5$ & $<0.5$ & $<1$ & $<0.5$ & $<0.5$ & $<2$ & $<1$ \\
\hline \multirow[t]{2}{*}{ Biokreisel } & STE & 434727121273702 & 20030604 & 1100 & $<0.5$ & 1.0 & E0.3 & $<1$ & $<0.5$ & $<0.5$ & E30 & $<1$ \\
\hline & DFMW & 434727121273701 & 20030604 & 1100 & $<0.5$ & $<0.5$ & $<0.5$ & $<1$ & $<0.5$ & $<0.5$ & $<2$ & $<1$ \\
\hline \multirow[t]{2}{*}{ EnviroServer } & STE & 434836121271102 & 20030423 & 1100 & $<0.5$ & E0.4 & $<0.5$ & $<1$ & $<0.5$ & $<0.5$ & $<2$ & M \\
\hline & DFMW & 434836121271101 & 20030423 & 1100 & $<0.5$ & $<0.5$ & $<0.5$ & $<1$ & $<0.5$ & $<0.5$ & $<2$ & $<1$ \\
\hline \multirow{2}{*}{ EnviroServer } & STE & 433855121300102 & 20030423 & 0900 & $<0.5$ & 0.5 & $<0.5$ & $<1$ & $<0.5$ & $<0.5$ & $<2$ & $<1$ \\
\hline & DFMW & 433855121300101 & 20030423 & 0900 & $<0.5$ & $<0.5$ & $<0.5$ & $<1$ & $<0.5$ & $<0.5$ & $<2$ & $<1$ \\
\hline \multirow[t]{2}{*}{ FAST } & STE & 434952121290602 & 20030407 & 1100 & $<0.5$ & 34 & E0.4 & $<1$ & $<0.5$ & $<0.5$ & 46 & 5 \\
\hline & DFMW & 434952121290601 & 20030407 & 1100 & $<0.5$ & $<0.5$ & $<0.5$ & $<1$ & $<0.5$ & $<0.5$ & E1 & $<1$ \\
\hline \multirow{2}{*}{ FAST } & STE & 434437121295302 & 20030505 & 0900 & $<0.5$ & 17 & $<0.5$ & $<1$ & $<0.5$ & $<0.5$ & 48 & $<1$ \\
\hline & DFMW & 434437121295301 & 20030507 & 0900 & $<0.5$ & $<0.5$ & $<0.5$ & $<1$ & $<0.5$ & $<0.5$ & $\mathrm{M}$ & $<1$ \\
\hline \multirow[t]{3}{*}{ NAYADIC } & STE & 435016121284702 & 20030407 & 1200 & $<5$ & 18 & 0.8 & $<5$ & $<5$ & $<5$ & E32 & $<5$ \\
\hline & LYS & 435016121284705 & 20030407 & 1200 & $<0.5$ & $<0.5$ & $<0.5$ & $<1$ & $<0.5$ & $<0.5$ & E2 & $<1$ \\
\hline & DFMW & 435016121284701 & 20030407 & 1200 & $<0.5$ & $<0.5$ & $<0.5$ & $<1$ & $<0.5$ & $<0.5$ & $<2$ & $<1$ \\
\hline \multirow[t]{2}{*}{ NAYADIC } & STE & 434713121274302 & 20030423 & 1000 & $<0.5$ & 9.0 & 0.9 & $<1$ & $<0.5$ & $<0.5$ & 15 & 11 \\
\hline & DFMW & 434713121274301 & 20030423 & 1000 & $<0.5$ & $<0.5$ & $<0.5$ & $<1$ & $<0.5$ & $<0.5$ & $<2$ & $<1$ \\
\hline \multirow[t]{2}{*}{ NITREX } & STE & 434203121311701 & 20030505 & 1000 & $<0.5$ & 4.7 & 4.1 & $<1$ & $<0.5$ & $<0.5$ & 16 & $<1$ \\
\hline & DFMW & 434203121311201 & 20030505 & 1000 & $<0.5$ & $<0.5$ & $<0.5$ & $<1$ & $<0.5$ & $<0.5$ & $<2$ & $<1$ \\
\hline \multirow[t]{2}{*}{ NITREX } & STE & 433824121340601 & 20030519 & 0900 & $<0.5$ & 12 & 0.6 & $<1$ & $<0.5$ & $<0.5$ & E36 & 3 \\
\hline & DFMW & 433825121340001 & 20030519 & 0900 & $<0.5$ & $<0.5$ & $<0.5$ & $<1$ & $<0.5$ & $<0.5$ & $<2$ & $<1$ \\
\hline \multirow[t]{2}{*}{ Puraflo } & STE & 434010121325602 & 20030519 & 0900 & $<0.5$ & 21 & 1.9 & $<1$ & $<0.5$ & $<0.5$ & E28 & 3 \\
\hline & DFMW & 434010121325601 & 20030519 & 0900 & $<0.5$ & $<0.5$ & $<0.5$ & $<1$ & $<0.5$ & $<0.5$ & $<2$ & $<1$ \\
\hline \multirow[t]{2}{*}{ Wert B } & STE & 434449121310202 & 20030514 & 1000 & $<0.5$ & 320 & 19 & $<1$ & $<0.5$ & $<0.5$ & 46 & 51 \\
\hline & DFMW & 434449121310201 & 20030514 & 1000 & $<0.5$ & $<0.5$ & $<0.5$ & $<1$ & $<0.5$ & $<0.5$ & $<2$ & $<1$ \\
\hline \multirow{2}{*}{ Wert B } & STE & 434423121312902 & 20030514 & 1000 & $<0.5$ & 68 & 1.9 & $<1$ & $<0.5$ & $<0.5$ & E16 & $<1$ \\
\hline & DFMW & 434423121312901 & 20030514 & 1000 & $<0.5$ & $<0.5$ & $<0.5$ & $<1$ & $<0.5$ & $<0.5$ & $\mathrm{M}$ & $<1$ \\
\hline
\end{tabular}


Table 6. Concentrations of organic wastewater compounds in water from traditional and innovative onsite wastewater treatment systems, downgradient lysimeters, and downgradient ground water, La Pine, Oregon, 2003.-Continued

[Benzophenone concentrations are reported in this table as they were reported by the laboratory, but are interpreted in the report relative to a project censoring level of 1 microgram per liter; see discussion of organic wastewater compounds in the results and discussion section of the text for explanation of this project censoring level; Type of onsite system, see descriptions in table 1; Sample Type: STE, septic tank effluent, LYS, lysimeter, DFMW, drainfield monitoring well; Date as year, month, day (YYYYMMDD); Time in hours and minutes, military; Chloride in milligrams per liter, from Oregon Department of Environmental Quality; Nitrite-plus-nitrate, and Total N, in milligrams N per liter, from Oregon Department of Environmental Quality; organic wastewater compounds in micrograms per liter; surrogate recoveries in percent; parameter codes for organic wastewater compound names: Pxxxx; "E", estimated; "<", less than; "M", presence verified, not quantified; Sample 434437121295301: organic wastewater compound sample collected 20030507, and chloride and nitrite-plus-nitrate samples collected 20030505; —, not reported (see text)]

\begin{tabular}{|c|c|c|c|c|c|c|c|c|c|c|c|c|}
\hline $\begin{array}{l}\text { system } \\
\end{array}$ & Sample type & Station number & Date & Time & (P39572) & (P38775) & (P62073) & (P34377)( & (P62075)( & (P62076) & (P62077) & (P34409) \\
\hline Standard & $\begin{array}{l}\text { STE } \\
\text { LYS } \\
\text { DFMW } \\
\text { STE } \\
\text { LYS } \\
\text { DFMW }\end{array}$ & $\begin{array}{l}434207121324602 \\
434207121324605 \\
434207121324601 \\
434236121310502 \\
434236121310505 \\
434236121310501 \\
\end{array}$ & $\begin{array}{l}20030414 \\
20030414 \\
20030414 \\
20030604 \\
20030604 \\
20030604 \\
\end{array}$ & $\begin{array}{l}0900 \\
0900 \\
0900 \\
0800 \\
0800 \\
0800 \\
\end{array}$ & $\begin{array}{l}<0.5 \\
<5 \\
<0.5 \\
<0.5 \\
<0.5 \\
<0.5\end{array}$ & $\begin{array}{l}<1 \\
<5 \\
<1 \\
<1 \\
<1 \\
<1\end{array}$ & $\begin{array}{l}\text { E2.8 } \\
<5 \\
<0.5 \\
<0.5 \\
<0.5 \\
<0.5\end{array}$ & $\begin{array}{l}\text { E0.1 } \\
<5 \\
<0.5 \\
<0.5 \\
<0.5 \\
<0.5\end{array}$ & $\begin{aligned} & 1.2 \\
&<5<0.5 \\
& 1.7 \\
& 1.2 \\
& \text { E0 } 0.1 \\
&\end{aligned}$ & $\begin{array}{l}220 \\
<5 \\
<0.5 \\
\text { E0.3 } \\
\text { E0.1 } \\
<0.5 \\
\end{array}$ & $\begin{array}{l}<0.5 \\
<5 \\
<0.5 \\
<0.5 \\
<0.5 \\
<0.5\end{array}$ & $\begin{array}{l}<0.5 \\
<5 \\
<0.5 \\
<0.5 \\
<0.5 \\
<0.5\end{array}$ \\
\hline $\begin{array}{l}\text { Sand Filter } \\
\text { Sand Filter }\end{array}$ & $\begin{array}{l}\text { STE } \\
\text { DFMW } \\
\text { STE } \\
\text { DFMW }\end{array}$ & $\begin{array}{l}434347121293902 \\
434347121293901 \\
434741121273401 \\
434741121273101\end{array}$ & $\begin{array}{l}20030414 \\
20030414 \\
20030407 \\
20030407 \\
\end{array}$ & $\begin{array}{l}1200 \\
1200 \\
1100 \\
1100\end{array}$ & $\begin{array}{l}<0.5 \\
<0.5 \\
<5 \\
<0.5\end{array}$ & $\begin{array}{l}<1 \\
<1 \\
<5 \\
<1\end{array}$ & $\begin{array}{l}\text { E1.9 } \\
<0.5 \\
\text { E0.8 } \\
<0.5\end{array}$ & $\begin{array}{l}\text { E0.5 } \\
<0.5 \\
<5 \\
<0.5\end{array}$ & $\begin{array}{r}10 \\
<0.5 \\
3.9 \\
<0.5 \\
\end{array}$ & $\begin{array}{r}72 \\
<0.5 \\
7.6 \\
<0.5 \\
\end{array}$ & $\begin{array}{l}<0.5 \\
<0.5 \\
<5 \\
<0.5\end{array}$ & $\begin{array}{l}<0.5 \\
<0.5 \\
<5 \\
<0.5\end{array}$ \\
\hline AdvanTex (AX-20 & $\begin{array}{l}\text { STE } \\
\text { DFMW }\end{array}$ & $\begin{array}{l}434536121291202 \\
434536121291201\end{array}$ & $\begin{array}{l}20030604 \\
20030604 \\
\end{array}$ & $\begin{array}{l}0900 \\
0900\end{array}$ & $\begin{array}{l}<0.5 \\
<0.5 \\
\end{array}$ & $\begin{array}{l}<1 \\
<1\end{array}$ & $\begin{array}{l}\text { E2.0 } \\
<0.5 \\
\end{array}$ & $\begin{array}{l}<0.5 \\
<0.5 \\
\end{array}$ & $\begin{array}{l}2.2 \\
\mathrm{M}\end{array}$ & $\begin{array}{r}0.9 \\
<0.5 \\
\end{array}$ & $\begin{array}{r}4.5 \\
<0.5 \\
\end{array}$ & $\begin{array}{l}<0.5 \\
<0.5\end{array}$ \\
\hline $\begin{array}{l}\text { Biokreisel } \\
\text { Biokreisel }\end{array}$ & $\begin{array}{l}\text { STE } \\
\text { DFMW } \\
\text { STE } \\
\text { DFMW } \\
\end{array}$ & $\begin{array}{l}434226121293302 \\
434226121293301 \\
434727121273702 \\
434727121273701 \\
\end{array}$ & $\begin{array}{l}20030414 \\
20030414 \\
20030604 \\
20030604 \\
\end{array}$ & $\begin{array}{l}1000 \\
1000 \\
1100 \\
1100 \\
\end{array}$ & $\begin{array}{l}<0.5 \\
<0.5 \\
\text { E0.3 } \\
<0.5 \\
\end{array}$ & $\begin{array}{l}<1 \\
<1 \\
<1 \\
<1\end{array}$ & $\begin{array}{l}\text { E0.2 } \\
<0.5 \\
\text { E1.1 } \\
<0.5 \\
\end{array}$ & $\begin{array}{l}<0.5 \\
<0.5 \\
\text { E0.1 } \\
<0.5 \\
\end{array}$ & $\begin{array}{r}5.6 \\
<0.5 \\
1.7 \\
<0.5 \\
\end{array}$ & $\begin{array}{l}4.1 \\
<0.5 \\
17 \\
<0.5 \\
\end{array}$ & $\begin{array}{r}<0.5 \\
<0.5 \\
0.8 \\
<0.5 \\
\end{array}$ & $\begin{array}{l}<0.5 \\
<0.5 \\
<0.5 \\
<0.5 \\
\end{array}$ \\
\hline $\begin{array}{l}\text { EnviroServer } \\
\text { EnviroServer }\end{array}$ & $\begin{array}{l}\text { STE } \\
\text { DFMW } \\
\text { STE } \\
\text { DFMW }\end{array}$ & $\begin{array}{l}434836121271102 \\
434836121271101 \\
433855121300102 \\
433855121300101\end{array}$ & $\begin{array}{l}20030423 \\
20030423 \\
20030423 \\
20030423 \\
\end{array}$ & $\begin{array}{l}1100 \\
1100 \\
0900 \\
0900 \\
\end{array}$ & $\begin{array}{l}<0.5 \\
<0.5 \\
<0.5 \\
<0.5\end{array}$ & $\begin{array}{l}<1 \\
<1 \\
<1 \\
<1\end{array}$ & $\begin{array}{l}<0.5 \\
<0.5 \\
\mathrm{M} \\
<0.5\end{array}$ & $\begin{array}{l}<0.5 \\
<0.5 \\
<0.5 \\
<0.5\end{array}$ & $\begin{array}{l}\text { E0.3 } \\
\text { E0.1 } \\
1.4 \\
M\end{array}$ & $\begin{array}{l}\text { E0.1 } \\
<0.5 \\
\mathrm{M} \\
<0.5 \\
\end{array}$ & $\begin{array}{l}<0.5 \\
<0.5 \\
<0.5 \\
<0.5\end{array}$ & $\begin{array}{l}<0.5 \\
<0.5 \\
<0.5 \\
<0.5\end{array}$ \\
\hline $\begin{array}{l}\text { NITREX } \\
\text { NITREX }\end{array}$ & $\begin{array}{l}\text { STE } \\
\text { DFMW } \\
\text { STE } \\
\text { DFMW }\end{array}$ & $\begin{array}{l}434203121311701 \\
434203121311201 \\
433824121340601 \\
433825121340001 \\
\end{array}$ & $\begin{array}{l}20030505 \\
20030505 \\
20030519 \\
20030519 \\
\end{array}$ & $\begin{array}{l}1000 \\
1000 \\
0900 \\
0900 \\
\end{array}$ & $\begin{array}{l}<0.5 \\
<0.5 \\
<0.5 \\
<0.5 \\
\end{array}$ & $\begin{array}{l}<1 \\
<1 \\
<1 \\
<1\end{array}$ & $\begin{array}{l}\text { E0.3 } \\
\text { M } \\
\text { E0.3 } \\
<0.5\end{array}$ & $\begin{array}{l}<0.5 \\
<0.5 \\
<0.5 \\
<0.5 \\
\end{array}$ & $\begin{array}{r}5.2 \\
\mathrm{E} 0.1 \\
1.2 \\
<0.5\end{array}$ & $\begin{array}{l}13 \\
<0.5 \\
23 \\
<0.5 \\
\end{array}$ & $\begin{array}{r}<0.5 \\
<0.5 \\
0.6 \\
<0.5 \\
\end{array}$ & $\begin{array}{l}<0.5 \\
<0.5 \\
<0.5 \\
<0.5\end{array}$ \\
\hline Puraflo & $\begin{array}{l}\text { STE } \\
\text { DFMW }\end{array}$ & $\begin{array}{l}434010121325602 \\
434010121325601\end{array}$ & $\begin{array}{l}20030519 \\
20030519 \\
\end{array}$ & $\begin{array}{l}0900 \\
0900 \\
\end{array}$ & $\begin{array}{l}<0.5 \\
<0.5\end{array}$ & $\begin{array}{l}<1 \\
<1\end{array}$ & $\begin{array}{l}\text { E1.1 } \\
<0.5\end{array}$ & $\begin{array}{l}<0.5 \\
<0.5\end{array}$ & $\begin{array}{r}2.5 \\
<0.5 \\
\end{array}$ & $\begin{array}{l}24 \\
<0.5\end{array}$ & $\begin{array}{r}3.5 \\
<0.5 \\
\end{array}$ & $\begin{array}{l}<0.5 \\
<0.5\end{array}$ \\
\hline Wert B & $\begin{array}{l}\text { STE } \\
\text { DFMW } \\
\text { STE } \\
\text { DFMW }\end{array}$ & $\begin{array}{l}434449121310202 \\
434449121310201 \\
434423121312902 \\
434423121312901 \\
\end{array}$ & $\begin{array}{l}20030514 \\
20030514 \\
20030514 \\
20030514 \\
\end{array}$ & $\begin{array}{l}1000 \\
1000 \\
1000 \\
1000 \\
\end{array}$ & $\begin{array}{l}<0.5 \\
<0.5 \\
<0.5 \\
<0.5 \\
\end{array}$ & $\begin{array}{l}<1 \\
<1 \\
<1 \\
<1\end{array}$ & $\begin{array}{l}\text { E2.9 } \\
<0.5 \\
<0.5 \\
<0.5 \\
\end{array}$ & $\begin{array}{l}<0.5 \\
<0.5 \\
<0.5 \\
<0.5\end{array}$ & $\begin{array}{l}18 \\
\mathrm{M} \\
4.4 \\
\mathrm{M}\end{array}$ & $\begin{array}{l}90 \\
<0.5 \\
11 \\
<0.5 \\
\end{array}$ & $\begin{array}{l}<0.5 \\
<0.5 \\
<0.5 \\
<0.5 \\
\end{array}$ & $\begin{array}{l}<0.5 \\
<0.5 \\
\text { E0.3 } \\
<0.5\end{array}$ \\
\hline
\end{tabular}


Table 6. Concentrations of organic wastewater compounds in water from traditional and innovative onsite wastewater treatment systems, downgradient lysimeters, and downgradient ground water, La Pine, Oregon, 2003.-Continued

[Benzophenone concentrations are reported in this table as they were reported by the laboratory, but are interpreted in the report relative to a project censoring level of 1 microgram per liter; see discussion of organic wastewater compounds in the results and discussion section of the text for explanation of this project censoring level; Type of onsite system, see descriptions in table 1; Sample Type: STE, septic tank effluent, LYS, lysimeter, DFMW, drainfield monitoring well; Date as year, month, day (YYYYMMDD); Time in hours and minutes, military; Chloride in milligrams per liter, from Oregon Department of Environmental Quality; Nitrite-plus-nitrate, and Total N, in milligrams N per liter, from Oregon Department of Environmental Quality; organic wastewater compounds in micrograms per liter; surrogate recoveries in percent; parameter codes for organic wastewater compound names: Pxxxx; "E", estimated; "<", less than; "M", presence verified, not quantified; Sample 434437121295301: organic wastewater compound sample collected 20030507, and chloride and nitrite-plus-nitrate samples collected 20030505; - , not reported (see text)]

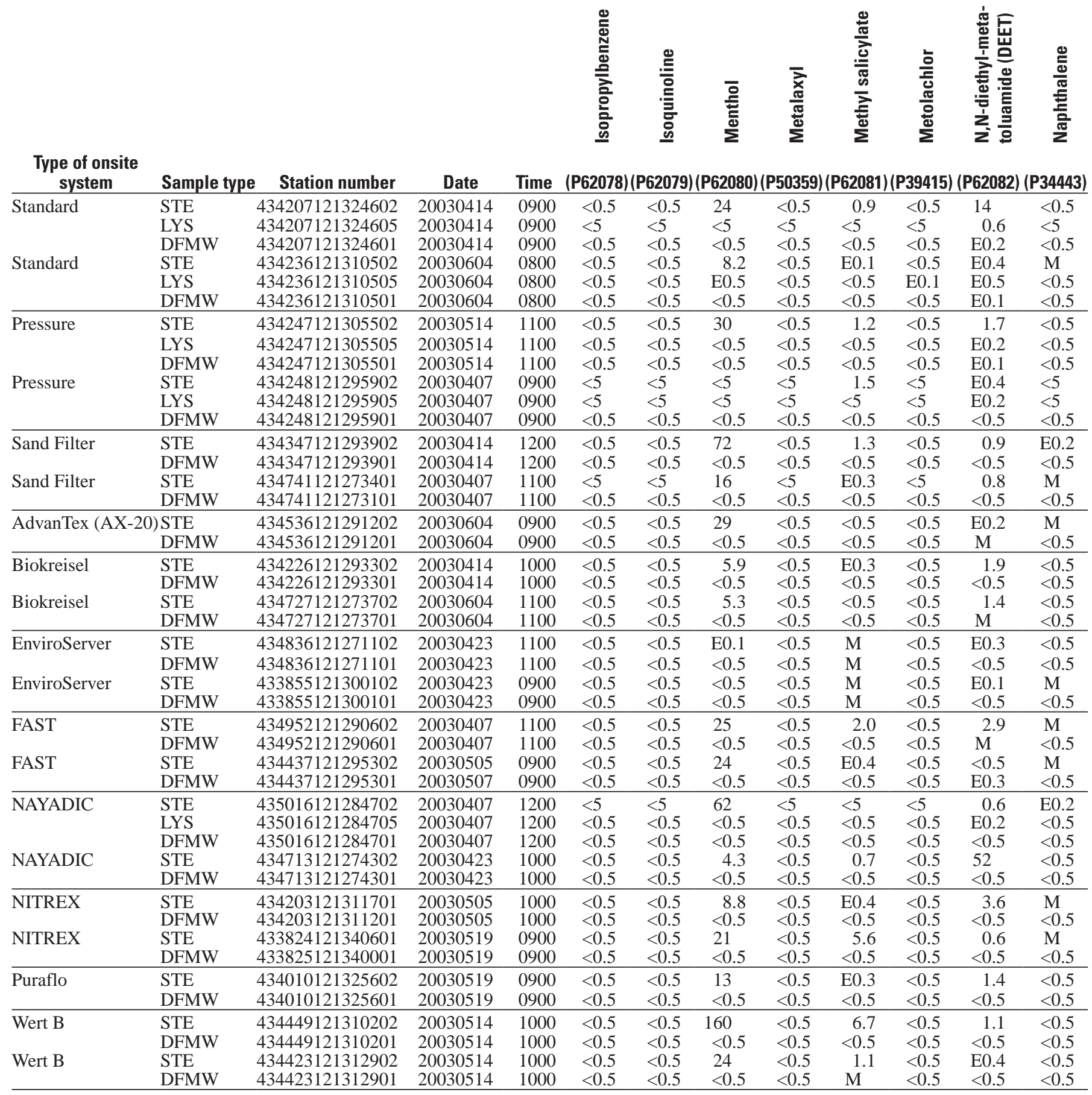


Table 6. Concentrations of organic wastewater compounds in water from traditional and innovative onsite wastewater treatment systems, downgradient lysimeters, and downgradient ground water, La Pine, Oregon, 2003.-Continued

[Benzophenone concentrations are reported in this table as they were reported by the laboratory, but are interpreted in the report relative to a project censoring level of 1 microgram per liter; see discussion of organic wastewater compounds in the results and discussion section of the text for explanation of this project censoring level; Type of onsite system, see descriptions in table 1; Sample Type: STE, septic tank effluent, LYS, lysimeter, DFMW, drainfield monitoring well; Date as year, month, day (YYYYMMDD); Time in hours and minutes, military; Chloride in milligrams per liter, from Oregon Department of Environmental Quality; Nitrite-plus-nitrate, and Total N, in milligrams N per liter, from Oregon Department of Environmental Quality; organic wastewater compounds in micrograms per liter; surrogate recoveries in percent; parameter codes for organic wastewater compound names: Pxxxx; "E", estimated; “<”, less than; "M", presence verified, not quantified; Sample 434437121295301: organic wastewater compound sample collected 20030507, and chloride and nitrite-plus-nitrate samples collected 20030505; —, not reported (see text)]

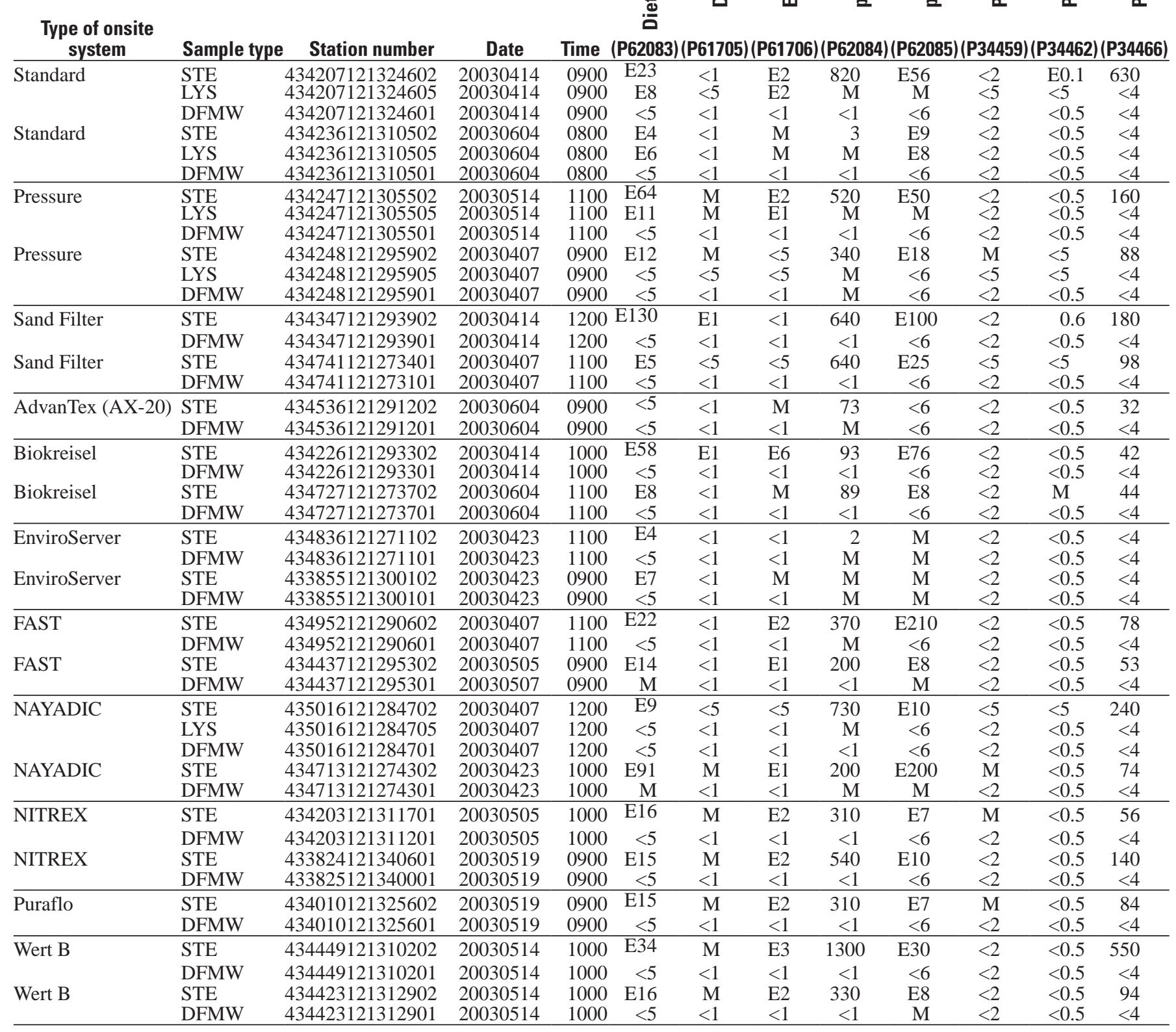


Table 6. Concentrations of organic wastewater compounds in water from traditional and innovative onsite wastewater treatment systems, downgradient lysimeters, and downgradient ground water, La Pine, Oregon, 2003.-Continued

[Benzophenone concentrations are reported in this table as they were reported by the laboratory, but are interpreted in the report relative to a project censoring level of 1 microgram per liter; see discussion of organic wastewater compounds in the results and discussion section of the text for explanation of this project censoring level; Type of onsite system, see descriptions in table 1; Sample Type: STE, septic tank effluent, LYS, lysimeter, DFMW, drainfield monitoring well; Date as year, month, day (YYYYMMDD); Time in hours and minutes, military; Chloride in milligrams per liter, from Oregon Department of Environmental Quality; Nitrite-plus-nitrate, and Total N, in milligrams N per liter, from Oregon Department of Environmental Quality; organic wastewater compounds in micrograms per liter; surrogate recoveries in percent; parameter codes for organic wastewater compound names: Pxxxx; "E", estimated; "<", less than; "M", presence verified, not quantified; Sample 434437121295301: organic wastewater compound sample collected 20030507, and chloride and nitrite-plus-nitrate samples collected 20030505; —, not reported (see text)]

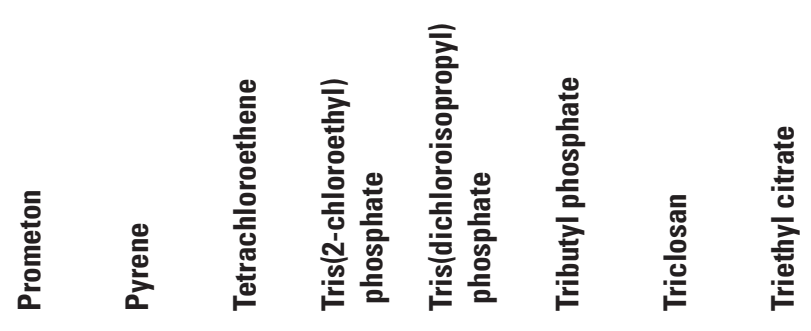

\begin{tabular}{|c|c|c|c|c|c|c|c|c|c|c|c|c|}
\hline $\begin{array}{c}\text { Type of onsite } \\
\text { system }\end{array}$ & Sample type & Station number & Date & Time (1 & $04037)$ & (P34470 & (P34476) & 62087 & (P62088) & (P62089) & )$(\mathrm{P} 620$ & P62091) \\
\hline \multirow[t]{2}{*}{ Standard } & $\begin{array}{l}\text { STE } \\
\text { LYS }\end{array}$ & $\begin{array}{l}434207121324602 \\
434207121324605\end{array}$ & $\begin{array}{l}20030414 \\
20030414\end{array}$ & $\begin{array}{l}0900 \\
0900\end{array}$ & $<0.5$ & $\underset{<5}{\mathrm{E} 0.1}$ & $\begin{array}{l}<0.5 \\
<5\end{array}$ & $\begin{array}{l}1.1 \\
1.4\end{array}$ & $\begin{array}{l}\text { E0.3 } \\
\text { E0.2 }\end{array}$ & $\begin{array}{l}<0.5 \\
\text { E0.2 }\end{array}$ & $\underset{<5}{\mathrm{M}}$ & $\begin{array}{r}0.7 \\
\mathrm{E} 0.1\end{array}$ \\
\hline & DFMW & 434207121324601 & 20030414 & 0900 & $<0.5$ & $<0.5$ & $<0.5$ & $<0.5$ & $<0.5$ & $<0.5$ & $<1$ & $<0.5$ \\
\hline \multirow[t]{3}{*}{ Standard } & STE & 434236121310502 & 20030604 & 0800 & $<0.5$ & $<0.5$ & $<0.5$ & E0.4 & $<0.5$ & E0.2 & E1 & E0.2 \\
\hline & LYS & 0505 & 20030604 & 0800 & $<0.5$ & $<0.5$ & $<0.5$ & E0.4 & $<0.5$ & E0.1 & M & E0.1 \\
\hline & DFMW & 4342 & 20030604 & 0800 & $<0.5$ & $<0.5$ & $<0.5$ & $<0.5$ & $<0.5$ & $<0.5$ & $\mathrm{M}$ & $<0.5$ \\
\hline \multirow[t]{3}{*}{ Pressure } & STE & 4342 & 20 & 1100 & & $<0.5$ & $<0.5$ & E0.3 & E0.4 & $<0.5$ & 3 & 4.5 \\
\hline & LYS & 05 & 20030 & 1100 & & $<0.5$ & $<0.5$ & E0.2 & E0.2 & E0.1 & $<1$ & E0.3 \\
\hline & DFMW & 501 & 20030514 & 1100 & $<0.5$ & $<0.5$ & $<0.5$ & E0.3 & $<0.5$ & $<0.5$ & M & $<0.5$ \\
\hline \multirow[t]{3}{*}{ Pressure } & STE & 5902 & 20030407 & 0900 & $<5$ & $<5$ & $<5$ & 0.6 & 0.6 & $<5$ & E3 & 0.7 \\
\hline & LYS & 905 & 20030407 & 0900 & $<5$ & $<5$ & $<5$ & E0.2 & E0.3 & E0.1 & $<5$ & E0.1 \\
\hline & DFMW & 434248121295901 & 20030407 & 0900 & $<0.5$ & $<0.5$ & $<0.5$ & $<0.5$ & $<0.5$ & $<0.5$ & $<1$ & $<0.5$ \\
\hline \multirow[t]{2}{*}{ Sand Filter } & STE & 4343 & 20030414 & 1200 & $<0.5$ & E0.3 & $<0.5$ & 0.5 & $<0.5$ & $<0.5$ & $<1$ & 1.0 \\
\hline & DFMW & 43 & 20 & 1200 & $<0.5$ & $<0.5$ & $<0.5$ & $<0.5$ & $<0.5$ & $<0.5$ & $<1$ & $<0.5$ \\
\hline \multirow[t]{2}{*}{ Sand Filter } & STE & 401 & 20030407 & 1100 & $<5$ & $<5$ & $<5$ & 1.2 & E0.4 & $<5$ & M & E0.3 \\
\hline & DFMW & 01 & 200 & 1100 & $<0.5$ & $<0.5$ & $<0.5$ & $<0.5$ & $<0.5$ & $<0.5$ & $<1$ & $<0.5$ \\
\hline \multirow{2}{*}{ AdvanTex (AX-20 } & STE & & & 0900 & $<0$ & $<0.5$ & $<0$. & E0.4 & E0. & $<0$ & 2 & E0.3 \\
\hline & DFMW & 4345 & 200 & 0900 & $<0$ & $<0.5$ & $<0.5$ & $<0.5$ & $<0.5$ & $<0.5$ & $\mathrm{M}$ & $<0.5$ \\
\hline \multirow[t]{2}{*}{ Biokreisel } & & & & 1000 & & $<0.5$ & $<05$ & E0.7 & E0.4 & E0.2 & $\mathrm{M}$ & E0.2 \\
\hline & $\mathrm{D}$ & 01 & & & & & & $<0.5$ & & & $<1$ & $<0.5$ \\
\hline \multirow{2}{*}{ Biokreisel } & STE & 702 & 04 & 1100 & $<$ & E0.1 & $<0.5$ & 0.8 & E0.5 & E0.4 & M & 1.3 \\
\hline & DF & 01 & 20 & 1100 & $<$ & $<0.5$ & E0.1 & $<0.5$ & $<0.5$ & $<0.5$ & $<1$ & $<0.5$ \\
\hline \multirow[t]{2}{*}{ EnviroServer } & STE & 02 & & 1100 & $<0$ & $<0.5$ & $<0.5$ & E0.2 & E0.1 & E0.1 & M & E0.2 \\
\hline & DFMW & 01 & 20 & 1100 & & $<0.5$ & $<0.5$ & $<0.5$ & M & $<0.5$ & M & $<0.5$ \\
\hline \multirow[t]{2}{*}{ EnviroServer } & STE & 102 & 200 & 0900 & $<0$ & $<0.5$ & $<0.5$ & E0.1 & E0.1 & $<0.5$ & M & E0.1 \\
\hline & DFM & 01 & 200 & 0900 & $<0$ & $<0.5$ & $<0.5$ & $<0.5$ & $<0.5$ & $<0.5$ & $\mathrm{M}$ & $<0.5$ \\
\hline \multirow[t]{2}{*}{ FAST } & STE & 02 & 200 & 1100 & $<0$ & $<0.5$ & $<0.5$ & E0.3 & E0.3 & 16 & E2 & 2.6 \\
\hline & DFMY & 01 & 20 & 1100 & $<0$ & $<0.5$ & $<0.5$ & E0.1 & E0.2 & E0.1 & $<1$ & $<0.5$ \\
\hline \multirow[t]{2}{*}{ FAST } & STE & 302 & 20 & 0900 & $<0$ & $<0.5$ & $<0.5$ & E0.2 & E0.2 & 1.7 & 1 & 0.9 \\
\hline & DFM & 01 & 20 & 0900 & $<0.5$ & $<0.5$ & $<0.5$ & E0.1 & E0.1 & E0.1 & $\mathrm{M}$ & $<0.5$ \\
\hline \multirow[t]{3}{*}{ NAYADIC } & STE & 702 & 2003 & 1200 & $<5$ & $<5$ & $<5$ & E0.4 & E0.4 & $<5$ & E2 & 0.6 \\
\hline & LYS & 05 & & 1200 & $<0.5$ & $<0.5$ & E0.2 & E0.3 & E0.2 & E0.2 & $<1$ & $<0.5$ \\
\hline & DFMI & 121284701 & 200 & 1200 & $<0$. & $<0.5$ & $<0.5$ & $<0.5$ & $<0.5$ & $<0.5$ & $<1$ & $<0.5$ \\
\hline \multirow[t]{2}{*}{ NAYADIC } & STE & 121274302 & 20030423 & 1000 & $<0.5$ & $<0.5$ & E0.1 & 1.1 & E0.2 & $<0.5$ & 4 & E0.3 \\
\hline & DFM & 4347 & 200304 & 1000 & $<0.5$ & $<0.5$ & $<0.5$ & $<0.5$ & $<0.5$ & $<0.5$ & $\mathrm{M}$ & $<0.5$ \\
\hline \multirow[t]{2}{*}{ NITREX } & STE & 01 & 20 & 1000 & $<0$. & $<0.5$ & $<0.5$ & E0.2 & E0.2 & 0.8 & 3 & E0.3 \\
\hline & DFMW & 434203121311201 & 20030 & 1000 & $<0$ & $<0$ & $\mathrm{M}$ & $<0.5$ & $<0.5$ & $<0.5$ & M & $<0.5$ \\
\hline \multirow{2}{*}{ NITREX } & STE & 433824121340601 & 20030519 & 0900 & $<0.5$ & $<0.5$ & $<0.5$ & 1.9 & E0.2 & $<0.5$ & 3 & 9.0 \\
\hline & DFM & 40001 & & 0900 & $<0.5$ & $<0.5$ & $<0.5$ & $<0.5$ & $<0.5$ & $<0.5$ & $<1$ & $<0.5$ \\
\hline \multirow[t]{2}{*}{ Puraflo } & STE & 434010121325602 & 20030519 & 0900 & $<0.5$ & $<0.5$ & $<0.5$ & E0.3 & E0.3 & E0.3 & 5 & E0.4 \\
\hline & & & & 0900 & $<0.5$ & $<0.5$ & $<0.5$ & $<0.5$ & $<0.5$ & $<0.5$ & $<1$ & $<0.5$ \\
\hline \multirow[t]{2}{*}{ Wert B } & STE & 434449121310202 & 20030514 & 1000 & $<0.5$ & $<0.5$ & $<0.5$ & 0.7 & 0.5 & $<0.5$ & 5 & 11 \\
\hline & DFM & & & & & & $<0.5$ & $<0.5$ & $<0.5$ & $<0.5$ & $\mathrm{M}$ & $<0.5$ \\
\hline \multirow[t]{2}{*}{ Wert B } & STE & 434423121312902 & 20030514 & 1000 & $<0.5$ & $<0.5$ & $<0.5$ & E0.3 & E0.4 & 1.6 & 5 & 0.7 \\
\hline & DFMW & 434423121312901 & 20030514 & 1000 & $<0.5$ & $<0.5$ & $<0.5$ & $<0.5$ & $<0.5$ & $<0.5$ & M & $<0.5$ \\
\hline
\end{tabular}


Table 6. Concentrations of organic wastewater compounds in water from traditional and innovative onsite wastewater treatment systems, downgradient lysimeters, and downgradient ground water, La Pine, Oregon, 2003.—Continued

[Benzophenone concentrations are reported in this table as they were reported by the laboratory, but are interpreted in the report relative to a project censoring level of 1 microgram per liter; see discussion of organic wastewater compounds in the results and discussion section of the text for explanation of this project censoring level; Type of onsite system, see descriptions in table 1; Sample Type: STE, septic tank effluent, LYS, lysimeter, DFMW, drainfield monitoring well; Date as year, month, day (YYYYMMDD); Time in hours and minutes, military; Chloride in milligrams per liter, from Oregon Department of Environmental Quality; Nitrite-plus-nitrate, and Total N, in milligrams N per liter, from Oregon Department of Environmental Quality; organic wastewater compounds in micrograms per liter; surrogate recoveries in percent; parameter codes for organic wastewater compound names: Pxxxx; "E", estimated; "<", less than; "M", presence verified, not quantified; Sample 434437121295301: organic wastewater compound sample collected 20030507, and chloride and nitrite-plus-nitrate samples collected 20030505; —, not reported (see text)]

\begin{tabular}{|c|c|c|c|c|c|c|c|c|c|c|}
\hline $\begin{array}{c}\text { Type of onsite } \\
\text { system }\end{array}$ & Sample type & Station number & Date & Time & (P62092) & (P62093) & (P99583) & (P99584) & (P99585) & (P99586) \\
\hline \multirow[t]{3}{*}{ Standard } & STE & 434207121324602 & 20030414 & 0900 & $\mathrm{E} 0.5$ & E5.1 & 71.1 & 82.7 & 64.0 & 79.7 \\
\hline & LYS & 434207121324605 & 20030414 & 0900 & $<5$ & E1.0 & 42.0 & 91.0 & 82.9 & 82.2 \\
\hline & DFMW & 434207121324601 & 20030414 & 0900 & $<0.5$ & $<0.5$ & 7.8 & 101 & 73.4 & 92.0 \\
\hline \multirow[t]{3}{*}{ Standard } & STE & 434236121310502 & 20030604 & 0800 & E0.2 & $<0.5$ & 141 & 115 & 94.9 & 97.4 \\
\hline & LYS & 434236121310505 & 20030604 & 0800 & E0.2 & $<0.5$ & 102 & 107 & 95.2 & 95.2 \\
\hline & DFMW & 434236121310501 & 20030604 & 0800 & $<0.5$ & $<0.5$ & 87.0 & 100 & 60.9 & 95.7 \\
\hline \multirow[t]{3}{*}{ Pressure } & STE & 434247121305502 & 20030514 & 1100 & E0.5 & 3.4 & 95.5 & 92.4 & 95.5 & 89.4 \\
\hline & LYS & 434247121305505 & 20030514 & 1100 & $<0.5$ & $<0.5$ & 86.5 & 97.3 & 78.4 & 86.5 \\
\hline & DFMW & 434247121305501 & 20030514 & 1100 & $<0.5$ & $<0.5$ & 59.1 & 86.4 & 95.5 & 90.9 \\
\hline \multirow{3}{*}{ Pressure } & STE & 434248121295902 & 20030407 & 0900 & 0.9 & E5.5 & 108 & 105 & 72.0 & 86.7 \\
\hline & LYS & 434248121295905 & 20030407 & 0900 & $<5$ & E0.5 & 54.2 & 98.1 & 66.4 & 85.4 \\
\hline & DFMW & 434248121295901 & 20030407 & 0900 & $<0.5$ & $<0.5$ & 44.4 & 94.1 & 72.5 & 87.8 \\
\hline \multirow[t]{2}{*}{ Sand Filter } & STE & 434347121293902 & 20030414 & 1200 & 0.5 & $<0.5$ & 72.6 & 97.5 & 68.6 & 88.5 \\
\hline & DFMW & 434347121293901 & 20030414 & 1200 & $<0.5$ & $<0.5$ & 0.0 & 89.3 & 59.1 & 81.9 \\
\hline \multirow[t]{2}{*}{ Sand Filter } & STE & 434741121273401 & 20030407 & 1100 & 0.6 & E1.9 & 80.6 & 89.5 & 75.4 & 78.3 \\
\hline & DFMW & 434741121273101 & 20030407 & 1100 & $<0.5$ & $<0.5$ & 13.7 & 87.5 & 76.7 & 86.8 \\
\hline \multirow[t]{2}{*}{$\overline{\text { AdvanTex }(A X-20)}$} & STE & 434536121291202 & 20030604 & 0900 & E0.2 & E0.9 & 119 & 111 & 94.4 & 103 \\
\hline & DFMW & 434536121291201 & 20030604 & 0900 & $<0.5$ & $<0.5$ & 90.9 & 105 & 54.5 & 100 \\
\hline \multirow[t]{2}{*}{ Biokreisel } & STE & 434226121293302 & 20030414 & 1000 & E0.4 & E2.4 & 87.1 & 93.2 & 65.1 & 88.0 \\
\hline & DFMW & 434226121293301 & 20030414 & 1000 & $<0.5$ & $<0.5$ & 0.0 & 92.0 & 63.7 & 84.2 \\
\hline \multirow{2}{*}{ Biokreisel } & STE & 434727121273702 & 20030604 & 1100 & E0.4 & E1.0 & 122 & 111 & 84.4 & 102 \\
\hline & DFMW & 434727121273701 & 20030604 & 1100 & $<0.5$ & $<0.5$ & 91.3 & 109 & 73.9 & 100 \\
\hline \multirow{2}{*}{ EnviroServer } & STE & 434836121271102 & 20030423 & 1100 & $\mathrm{M}$ & $\mathrm{M}$ & 78.1 & 87.5 & 68.8 & 75.0 \\
\hline & DFMW & 434836121271101 & 20030423 & 1100 & $<0.5$ & $<0.5$ & 41.7 & 82.6 & 56.5 & 73.9 \\
\hline \multirow{2}{*}{ EnviroServer } & STE & 433855121300102 & 20030423 & 0900 & E0.1 & E0.3 & 85.2 & 85.2 & 74.1 & 74.1 \\
\hline & DFMW & 433855121300101 & 20030423 & 0900 & $<0.5$ & $<0.5$ & 13.2 & 81.8 & 59.1 & 72.7 \\
\hline \multirow[t]{2}{*}{$\overline{\text { FAST }}$} & STE & 434952121290602 & 20030407 & 1100 & E0.4 & E2.8 & 65.4 & 91.6 & 74.4 & 86.0 \\
\hline & DFMW & 434952121290601 & 20030407 & 1100 & $<0.5$ & $<0.5$ & 47.8 & 92.3 & 66.3 & 83.9 \\
\hline \multirow[t]{2}{*}{ FAST } & STE & 434437121295302 & 20030505 & 0900 & $\mathrm{E} 0.3$ & 1.6 & 110 & 127 & 90.2 & 124 \\
\hline & DFMW & 434437121295301 & 20030507 & 0900 & $<0.5$ & $<0.5$ & 87.0 & 122 & 113 & 117 \\
\hline \multirow[t]{3}{*}{ NAYADIC } & STE & 435016121284702 & 20030407 & 1200 & 0.8 & E2.2 & 78.6 & 133 & 59.3 & 81.1 \\
\hline & LYS & 435016121284705 & 20030407 & 1200 & $<0.5$ & $<0.5$ & 31.7 & 95.3 & 60.6 & 84.2 \\
\hline & DFMW & 435016121284701 & 20030407 & 1200 & $<0.5$ & $<0.5$ & 0.0 & 83.8 & 72.1 & 82.6 \\
\hline \multirow[t]{2}{*}{ NAYADIC } & STE & 434713121274302 & 20030423 & 1000 & E0.4 & 1.8 & 70.2 & 83.0 & 68.1 & 76.6 \\
\hline & DFMW & 434713121274301 & 20030423 & 1000 & $<0.5$ & $<0.5$ & 44.5 & 81.8 & 59.1 & 72.7 \\
\hline \multirow[t]{2}{*}{ NITREX } & STE & 434203121311701 & 20030505 & 1000 & E0.3 & 2.8 & 125 & 129 & 88.2 & 106 \\
\hline & DFMW & 434203121311201 & 20030505 & 1000 & $<0.5$ & $<0.5$ & 27.7 & 132 & 86.4 & 109 \\
\hline \multirow{2}{*}{ NITREX } & STE & 433824121340601 & 20030519 & 0900 & E0.2 & 1.7 & 70.4 & 98.1 & 77.8 & 88.9 \\
\hline & DFMW & 433825121340001 & 20030519 & 0900 & $<0.5$ & $<0.5$ & 43.5 & 87.0 & 69.6 & 87.0 \\
\hline \multirow[t]{2}{*}{ Puraflo } & STE & 434010121325602 & 20030519 & 0900 & 0.8 & 6.1 & 87.5 & 97.9 & 87.5 & 87.5 \\
\hline & DFMW & 434010121325601 & 20030519 & 0900 & $<0.5$ & $<0.5$ & 43.2 & 86.4 & 63.6 & 90.9 \\
\hline \multirow[t]{2}{*}{ Wert B } & STE & 434449121310202 & 20030514 & 1000 & 0.8 & 16 & 90.9 & 90.9 & 88.2 & 86.4 \\
\hline & DFMW & 434449121310201 & 20030514 & 1000 & $<0.5$ & $<0.5$ & 52.4 & 95.2 & 85.7 & 100 \\
\hline \multirow[t]{2}{*}{ Wert B } & STE & 434423121312902 & 20030514 & 1000 & E0.2 & $<0.5$ & 83.0 & 96.2 & 90.6 & 90.6 \\
\hline & DFMW & 434423121312901 & 20030514 & 1000 & $<0.5$ & $<0.5$ & 43.2 & 90.9 & 90.9 & 95.5 \\
\hline
\end{tabular}


Table 7. Concentrations of organic wastewater compounds in ground water along transects, La Pine, Oregon, 2003.

[Benzophenone concentrations are reported in this table as they were reported by the laboratory, but are interpreted in the report relative to a project censoring level of 1 microgram per liter; see discussion of organic wastewater compounds in the results and discussion section of the text for explanation of this project censoring level; Date as year, month, day (YYYYMMDD); Time in hours and minutes, military; Chloride in milligrams per liter; Nitrite-plus-nitrate in milligrams N per liter; D.O., dissolved oxygen, in milligrams per liter; Specific conductance in microsiemens per centimeter at 25 degrees Celsius; Temperature in degrees Celsius; Organic wastewater compounds in micrograms per liter; surrogate recoveries in percent; parameter codes for organic wastewater compound names: Pxxxx; "E", estimated; "<", less than; "M", presence verified, not quantified; - , no data]

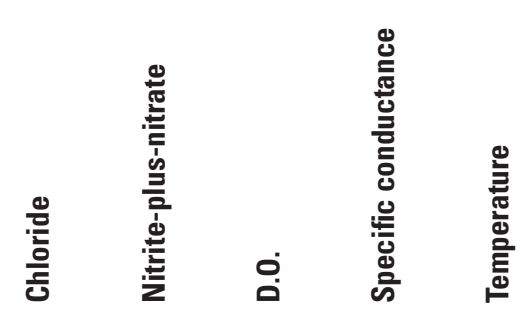

Station number Site name Type of sample

Date Time (P00940) (P00631) (P00300) (P00095) (P00010)

Senior Center Transect

\begin{tabular}{|c|c|c|c|c|c|c|c|c|c|}
\hline 434212121294299 & Senior Septic Tank & Septic Tank Effluent & 20030430 & 1000 & - & - & - & - & - \\
\hline 434212121294201 & Senior 1 & Ground water & 20030429 & 1100 & 5.22 & 1.94 & 6.7 & 141 & 9.1 \\
\hline 434212121294202 & Senior 2 & Ground water & 20030429 & 1300 & 6.26 & 4.48 & 6.2 & 243 & 9.6 \\
\hline 434212121294203 & Senior 3 & Ground water & 20030430 & 1300 & 14.5 & 15.9 & 5.3 & 381 & 10.1 \\
\hline 434212121294204 & Senior 4 & Ground water & 20030430 & 1600 & 5.71 & 4.79 & 6.2 & 164 & 10.1 \\
\hline 434212121294205 & Senior 5 & Ground water & 20030430 & 1800 & 16.6 & 17.8 & 6.5 & 381 & 9.6 \\
\hline 434212121294206 & Senior 6 & Ground water & 20030501 & 1000 & 6.40 & 4.82 & 5.8 & 193 & 10.6 \\
\hline 434212121294207 & Senior 7 & Ground water & 20030501 & 1300 & 1.79 & 0.65 & 6.8 & 93 & 10.8 \\
\hline 434212121294208 & Senior 8 & Ground water & 20030501 & 1500 & 12.6 & 13.4 & 4.5 & 342 & 11.5 \\
\hline 434212121294209 & Senior 9 & Ground water & 20030501 & 1700 & 1.09 & 0.11 & 6.2 & 74 & 10.6 \\
\hline 434212121294210 & Senior 10 & Ground water & 20030502 & 1100 & 3.30 & 1.55 & 7.9 & 119 & 10.0 \\
\hline 434212121294211 & Senior 11 & Ground water & 20030502 & 1200 & 1.78 & 0.27 & 7.4 & 85 & 10.6 \\
\hline \multicolumn{10}{|c|}{ High Lakes Church Transect } \\
\hline 434241121311601 & High Lakes 1 & Ground water & 20030610 & 1600 & 3.12 & 0.49 & 1.8 & 144 & 11.3 \\
\hline 434241121311602 & High Lakes 2 & Ground water & 20030610 & 1800 & 26.3 & 19.0 & 5.3 & 355 & 9.6 \\
\hline 434241121311603 & High Lakes 3 & Ground water & 20030611 & 1200 & 14.0 & $<0.02$ & 0.3 & 248 & 10.2 \\
\hline 434241121311604 & High Lakes 4 & Ground water & 20030611 & 1500 & 4.78 & 0.29 & 0.5 & 174 & 10.3 \\
\hline 434241121311605 & High Lakes 5 & Ground water & 20030611 & 1600 & 7.92 & 3.57 & 3.9 & 161 & 10.4 \\
\hline 434241121311606 & High Lakes 6 & Ground water & 20030612 & 1300 & 19.6 & 10.6 & 7.9 & 256 & 10.5 \\
\hline 434241121311607 & High Lakes 7 & Ground water & 20030612 & 1500 & 19.6 & 10.3 & 7.7 & 270 & 11.7 \\
\hline 434241121311608 & High Lakes 8 & Ground water & 20030612 & 1900 & 1.73 & 0.72 & 3.2 & 90 & 10.4 \\
\hline 434241121311609 & High Lakes 9 & Ground water & 20030613 & 1100 & 1.98 & 0.11 & 5.4 & 93 & 7.8 \\
\hline \multicolumn{10}{|c|}{ Pine Forest Road Transect } \\
\hline 434210121313401 & Pine 1 & Ground water & 20030609 & 1200 & 51.0 & 35.5 & - & 586 & 13.9 \\
\hline 434210121313402 & Pine 2 & Ground water & 20030609 & 1400 & 8.18 & 4.80 & 7.2 & 193 & 12.0 \\
\hline 434210121313403 & Pine 3 & Ground water & 20030609 & 1600 & 10.1 & 5.86 & 8.3 & 205 & 10.0 \\
\hline 434210121313404 & Pine 4 & Ground water & 20030609 & 1800 & 37.0 & 25.6 & 7.0 & 460 & 10.8 \\
\hline 434210121313405 & Pine 5 & Ground water & 20030616 & 1300 & 4.44 & 2.03 & 8.2 & 155 & 11.6 \\
\hline 434210121313406 & Pine 6 & Ground water & 20030616 & 1500 & 10.4 & 6.22 & 8.4 & 203 & 11.4 \\
\hline 434210121313407 & Pine 7 & Ground water & 20030617 & 1100 & 5.03 & 2.63 & 6.6 & 155 & 10.6 \\
\hline 434210121313408 & Pine 8 & Ground water & 20030617 & 1300 & 12.9 & 7.63 & 8.3 & 241 & 10.4 \\
\hline 434210121313409 & Pine 9 & Ground water & 20030617 & 1500 & 18.3 & 12.4 & 7.8 & 304 & 11.1 \\
\hline 434210121313410 & Pine 10 & Ground water & 20030619 & 1000 & 10.6 & 5.19 & 8.8 & 199 & 9.1 \\
\hline$\underline{434210121313411}$ & Pine 11 & Ground water & 20030619 & 1200 & 39.8 & 30.4 & 7.9 & 524 & 9.4 \\
\hline
\end{tabular}


Table 7. Concentrations of organic wastewater compounds in ground water along transects, La Pine, Oregon, 2003.—Continued

[[Benzophenone concentrations are reported in this table as they were reported by the laboratory, but are interpreted in the report relative to a project censoring level of 1 microgram per liter; see discussion of organic wastewater compounds in the results and discussion section of the text for explanation of this project censoring level; Date as year, month, day (YYYYMMDD); Time in hours and minutes, military; Chloride in milligrams per liter; Nitriteplus-nitrate in milligrams N per liter; D.O., dissolved oxygen, in milligrams per liter; Specific conductance in microsiemens per centimeter at 25 degrees Celsius; Temperature in degrees Celsius; Organic wastewater compounds in micrograms per liter; surrogate recoveries in percent; parameter codes for organic wastewater compound names: Pxxxx; "E”, estimated; "<”, less than; "M", presence verified, not quantified; —, no data]

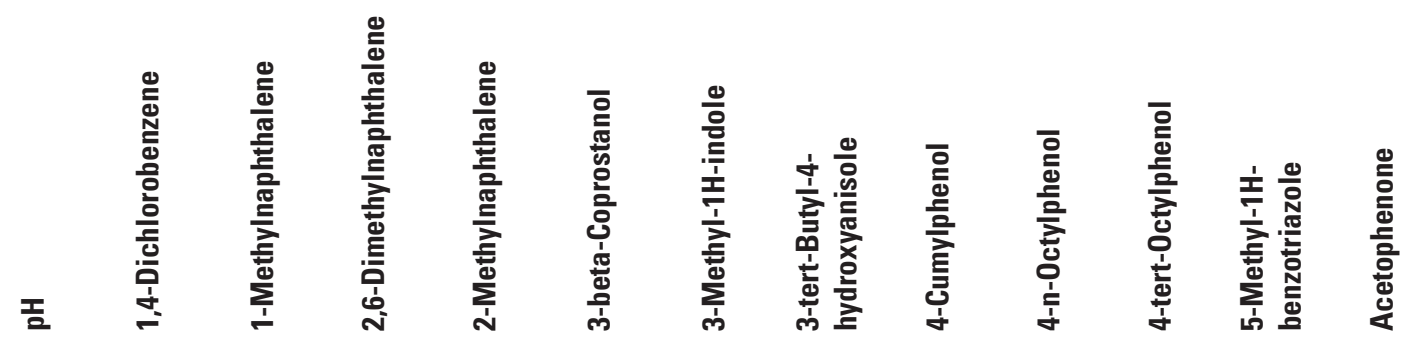

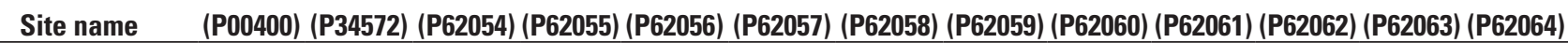

\begin{tabular}{|c|c|c|c|c|c|c|c|c|c|c|c|c|c|}
\hline \multicolumn{14}{|c|}{ Senior Center Transect } \\
\hline Senior Septic Tank & - & E0.3 & $\mathrm{M}$ & $<0.5$ & E0.1 & 2 & 21 & $\mathrm{M}$ & $<1$ & $<1$ & $<1$ & E2 & E0.4 \\
\hline Senior 1 & 7.0 & $<0.5$ & $<0.5$ & $<0.5$ & $<0.5$ & $<2$ & $<1$ & $<5$ & $<1$ & $<1$ & $<1$ & $<2$ & $<0.5$ \\
\hline Senior 2 & 7.1 & $<0.5$ & $<0.5$ & $<0.5$ & $<0.5$ & $<2$ & $\mathrm{M}$ & $<5$ & $<1$ & $<1$ & $<1$ & $<2$ & $<0.5$ \\
\hline Senior 3 & 7.1 & $<0.5$ & $<0.5$ & $<0.5$ & $<0.5$ & $<2$ & $<1$ & $<5$ & $<1$ & $<1$ & $<1$ & $<2$ & $<0.5$ \\
\hline Senior 4 & 7.4 & $<0.5$ & $<0.5$ & $<0.5$ & $<0.5$ & $<2$ & $<1$ & $<5$ & $<1$ & $<1$ & $<1$ & $<2$ & $<0.5$ \\
\hline Senior 5 & 7.2 & $<0.5$ & $<0.5$ & $<0.5$ & $<0.5$ & $<2$ & $<1$ & $<5$ & $<1$ & $<1$ & $<1$ & $<2$ & $<0.5$ \\
\hline Senior 6 & 7.1 & $<0.5$ & $<0.5$ & $<0.5$ & $<0.5$ & $<2$ & $<1$ & $<5$ & $<1$ & $<1$ & $<1$ & $<2$ & $<0.5$ \\
\hline Senior 7 & 7.5 & $<0.5$ & $<0.5$ & $<0.5$ & $<0.5$ & $<2$ & $<1$ & $<5$ & $<1$ & $<1$ & $<1$ & $<2$ & $<0.5$ \\
\hline Senior 8 & 6.8 & $<0.5$ & $<0.5$ & $<0.5$ & $<0.5$ & $<2$ & $<1$ & $<5$ & $<1$ & $<1$ & $<1$ & $<2$ & $<0.5$ \\
\hline Senior 9 & 7.5 & $<0.5$ & $<0.5$ & $<0.5$ & $<0.5$ & $<2$ & $<1$ & $<5$ & $<1$ & $<1$ & $<1$ & $<2$ & $<0.5$ \\
\hline Senior 10 & 7.2 & $<0.5$ & $<0.5$ & $<0.5$ & $<0.5$ & $<2$ & $<1$ & $<5$ & $<1$ & $<1$ & $<1$ & $<2$ & $<0.5$ \\
\hline$\underline{\text { Senior } 11}$ & 7.4 & $<0.5$ & $<0.5$ & $<0.5$ & $<0.5$ & $<2$ & $<1$ & $<5$ & $<1$ & $<1$ & $<1$ & $<2$ & $<0.5$ \\
\hline \multicolumn{14}{|c|}{ High Lakes Church Transect } \\
\hline High Lakes 1 & 7.2 & $\mathrm{M}$ & $<0.5$ & $<0.5$ & $<0.5$ & $<2$ & $<1$ & $<5$ & $<1$ & $<1$ & $<1$ & $<2$ & $<0.5$ \\
\hline High Lakes 2 & 6.9 & M & $<0.5$ & $<0.5$ & $<0.5$ & $<2$ & $<1$ & $<5$ & $<1$ & $<1$ & $<1$ & $<2$ & $<0.5$ \\
\hline High Lakes 3 & 7.2 & $<0.5$ & $<0.5$ & $<0.5$ & $<0.5$ & $<2$ & $<1$ & $<5$ & $<1$ & $<1$ & $<1$ & $<2$ & $<0.5$ \\
\hline High Lakes 4 & 7.3 & $\mathrm{M}$ & $<0.5$ & $<0.5$ & $<0.5$ & $<2$ & $<1$ & $<5$ & $<1$ & $<1$ & $<1$ & $<2$ & $<0.5$ \\
\hline High Lakes 5 & 6.9 & M & $<0.5$ & $<0.5$ & $<0.5$ & $<2$ & $<1$ & $<5$ & $<1$ & $<1$ & $<1$ & $<2$ & $<0.5$ \\
\hline High Lakes 6 & 6.8 & M & $<0.5$ & $<0.5$ & $<0.5$ & $<2$ & $<1$ & $<5$ & $<1$ & $<1$ & $<1$ & $<2$ & $<0.5$ \\
\hline High Lakes 7 & 6.6 & $<0.5$ & $<0.5$ & $<0.5$ & $<0.5$ & $<2$ & $<1$ & $<5$ & $<1$ & $<1$ & $<1$ & $<2$ & $<0.5$ \\
\hline High Lakes 8 & 7.1 & $<0.5$ & $<0.5$ & $<0.5$ & $<0.5$ & $<2$ & $<1$ & $<5$ & $<1$ & $<1$ & $<1$ & $<2$ & $<0.5$ \\
\hline High Lakes 9 & 7.3 & $<0.5$ & $<0.5$ & $<0.5$ & $<0.5$ & $<2$ & $<1$ & $<5$ & $<1$ & $<1$ & $<1$ & $<2$ & $<0.5$ \\
\hline \multicolumn{14}{|c|}{ Pine Forest Road Transect } \\
\hline Pine 1 & 6.6 & $\mathrm{M}$ & $<0.5$ & $<0.5$ & $<0.5$ & $<2$ & $<1$ & $<5$ & $<1$ & $<1$ & $<1$ & $<2$ & $<0.5$ \\
\hline Pine 2 & 6.8 & $<0.5$ & $<0.5$ & $<0.5$ & $<0.5$ & $<2$ & $<1$ & $<5$ & $<1$ & $<1$ & $<1$ & $<2$ & $<0.5$ \\
\hline Pine 3 & 6.8 & $<0.5$ & $<0.5$ & $<0.5$ & $<0.5$ & $<2$ & $<1$ & $<5$ & $<1$ & $<1$ & $<1$ & $<2$ & $<0.5$ \\
\hline Pine 4 & 6.5 & M & $<0.5$ & $<0.5$ & $<0.5$ & $<2$ & $<1$ & $<5$ & $<1$ & $<1$ & $<1$ & $<2$ & $<0.5$ \\
\hline Pine 5 & 6.8 & $<0.5$ & $<0.5$ & $<0.5$ & $<0.5$ & $<2$ & $<1$ & $<5$ & $<1$ & $<1$ & $<1$ & $<2$ & $<0.5$ \\
\hline Pine 6 & 6.4 & $<0.5$ & $<0.5$ & $<0.5$ & $<0.5$ & $<2$ & $<1$ & $<5$ & $<1$ & $<1$ & $<1$ & $<2$ & $<0.5$ \\
\hline Pine 7 & 6.9 & $<0.5$ & $<0.5$ & $<0.5$ & $<0.5$ & $<2$ & $<1$ & $<5$ & $<1$ & $<1$ & $<1$ & $<2$ & $<0.5$ \\
\hline Pine 8 & 6.6 & $<0.5$ & $<0.5$ & $<0.5$ & $<0.5$ & $<2$ & $<1$ & $<5$ & $<1$ & $<1$ & $<1$ & $<2$ & $<0.5$ \\
\hline Pine 9 & 6.4 & $<0.5$ & $<0.5$ & $<0.5$ & $<0.5$ & $<2$ & $<1$ & $<5$ & $<1$ & $<1$ & $<1$ & $<2$ & $<0.5$ \\
\hline Pine 10 & 6.9 & $<0.5$ & $<0.5$ & $<0.5$ & $<0.5$ & $<2$ & $<1$ & $<5$ & $<1$ & $<1$ & $<1$ & $<2$ & $<0.5$ \\
\hline Pine 11 & 6.7 & $\mathrm{M}$ & $<0.5$ & $<0.5$ & $<0.5$ & $<2$ & $<1$ & $<5$ & $<1$ & $<1$ & $<1$ & $<2$ & $<0.5$ \\
\hline
\end{tabular}


Table 7. Concentrations of organic wastewater compounds in ground water along transects, La Pine, Oregon, 2003.—Continued

[Benzophenone concentrations are reported in this table as they were reported by the laboratory, but are interpreted in the report relative to a project censoring level of 1 microgram per liter; see discussion of organic wastewater compounds in the results and discussion section of the text for explanation of this project censoring level; Date as year, month, day (YYYYMMDD); Time in hours and minutes, military; Chloride in milligrams per liter; Nitriteplus-nitrate in milligrams N per liter; D.O., dissolved oxygen, in milligrams per liter; Specific conductance in microsiemens per centimeter at 25 degrees Celsius; Temperature in degrees Celsius; Organic wastewater compounds in micrograms per liter; surrogate recoveries in percent; parameter codes for organic wastewater compound names: Pxxxx; "E", estimated; "<", less than; "M", presence verified, not quantified; —, no data]

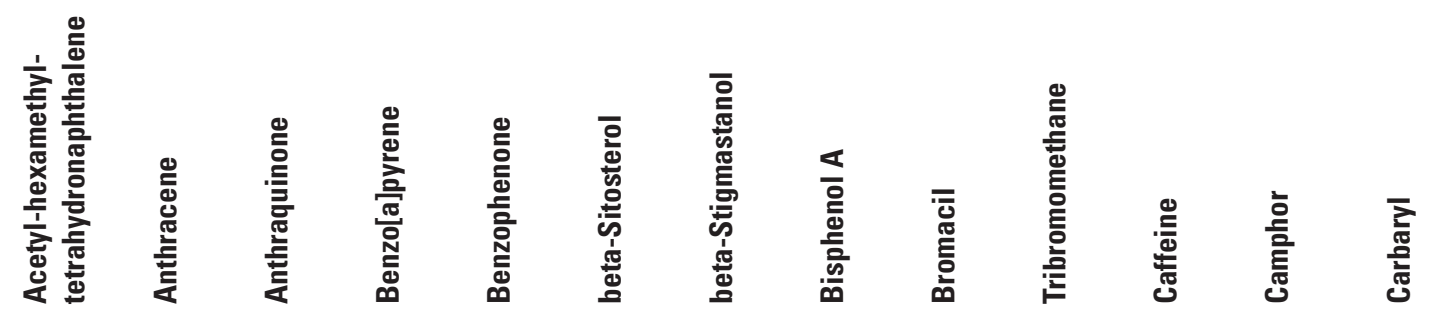

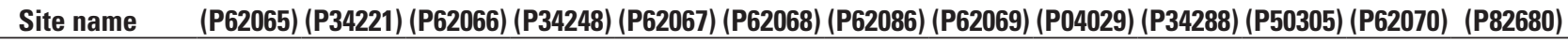

Senior Center Transect

\begin{tabular}{|c|c|c|c|c|c|c|c|c|c|c|c|c|c|}
\hline Senior Septic Tank & E0.1 & $<0.5$ & $<0.5$ & $<0.5$ & 0.8 & E4 & E3 & $\mathrm{M}$ & $<0.5$ & $<0.5$ & 170 & 2.8 & $<1$ \\
\hline Senior 1 & E0.1 & $<0.5$ & $<0.5$ & $<0.5$ & E0.1 & $<2$ & $<2$ & $<1$ & $<0.5$ & $<0.5$ & M & $<0.5$ & $<1$ \\
\hline Senior 2 & $<0.5$ & $<0.5$ & $<0.5$ & $<0.5$ & $<0.5$ & $<2$ & $<2$ & $<1$ & $<0.5$ & $<0.5$ & E0.4 & $<0.5$ & $<1$ \\
\hline Senior 3 & $<0.5$ & $<0.5$ & $<0.5$ & $<0.5$ & E0.1 & $<2$ & $<2$ & $<1$ & $<0.5$ & $<0.5$ & M & $<0.5$ & $<1$ \\
\hline Senior 4 & $<0.5$ & $<0.5$ & $<0.5$ & $<0.5$ & $<0.5$ & $<2$ & $<2$ & $<1$ & $<0.5$ & $<0.5$ & $<0.5$ & $<0.5$ & $<1$ \\
\hline Senior 5 & $<0.5$ & $<0.5$ & $<0.5$ & $<0.5$ & $<0.5$ & $<2$ & $<2$ & $<1$ & $<0.5$ & $<0.5$ & $<0.5$ & $<0.5$ & $<1$ \\
\hline Senior 6 & $<0.5$ & $<0.5$ & $<0.5$ & $<0.5$ & $<0.5$ & $<2$ & $<2$ & M & $<0.5$ & $<0.5$ & $<0.5$ & $<0.5$ & $<1$ \\
\hline Senior 7 & $<0.5$ & $<0.5$ & $<0.5$ & $<0.5$ & $<0.5$ & $<2$ & $<2$ & $<1$ & $<0.5$ & $<0.5$ & $<0.5$ & $<0.5$ & $<1$ \\
\hline Senior 8 & $<0.5$ & $<0.5$ & $<0.5$ & $<0.5$ & $<0.5$ & $<2$ & $<2$ & $<1$ & $<0.5$ & $<0.5$ & $<0.5$ & $<0.5$ & $<1$ \\
\hline Senior 9 & $<0.5$ & $<0.5$ & $<0.5$ & $<0.5$ & $<0.5$ & $<2$ & $<2$ & $<1$ & $<0.5$ & $<0.5$ & $<0.5$ & $<0.5$ & $<1$ \\
\hline Senior 10 & $<0.5$ & $<0.5$ & $<0.5$ & $<0.5$ & $<0.5$ & $<2$ & $<2$ & $<1$ & $<0.5$ & $<0.5$ & M & $<0.5$ & $<1$ \\
\hline Senior 11 & $<0.5$ & $<0.5$ & $<0.5$ & $<0.5$ & $<0.5$ & $<2$ & $<2$ & $<1$ & $<0.5$ & $<0.5$ & $<0.5$ & $<0.5$ & $<1$ \\
\hline \multicolumn{14}{|c|}{ High Lakes Church Transect } \\
\hline High Lakes 1 & $<0.5$ & $<0.5$ & $<0.5$ & $<0.5$ & $<0.5$ & $<2$ & $<2$ & $<1$ & $<0.5$ & $<0.5$ & $<0.5$ & $<0.5$ & $<1$ \\
\hline High Lakes 2 & $<0.5$ & $<0.5$ & $<0.5$ & $<0.5$ & $<0.5$ & $<2$ & $<2$ & M & $<0.5$ & $<0.5$ & $<0.5$ & $<0.5$ & $<1$ \\
\hline High Lakes 3 & $<0.5$ & $<0.5$ & $<0.5$ & $<0.5$ & $<0.5$ & $<2$ & $<2$ & $<1$ & $<0.5$ & $<0.5$ & $<0.5$ & $<0.5$ & $<1$ \\
\hline High Lakes 4 & $<0.5$ & $<0.5$ & $<0.5$ & $<0.5$ & $<0.5$ & $<2$ & $<2$ & $<1$ & $<0.5$ & $<0.5$ & $<0.5$ & $<0.5$ & $<1$ \\
\hline High Lakes 5 & $<0.5$ & $<0.5$ & $<0.5$ & $<0.5$ & $<0.5$ & $<2$ & $<2$ & $<1$ & $<0.5$ & $<0.5$ & $<0.5$ & $<0.5$ & $<1$ \\
\hline High Lakes 6 & $<0.5$ & $<0.5$ & $<0.5$ & $<0.5$ & $<0.5$ & $<2$ & $<2$ & $<1$ & $<0.5$ & $<0.5$ & $<0.5$ & $<0.5$ & $<1$ \\
\hline High Lakes 7 & $<0.5$ & $<0.5$ & $<0.5$ & $<0.5$ & $<0.5$ & $<2$ & $<2$ & $<1$ & $<0.5$ & $<0.5$ & $<0.5$ & $<0.5$ & $<1$ \\
\hline High Lakes 8 & $<0.5$ & $<0.5$ & $<0.5$ & $<0.5$ & $<0.5$ & $<2$ & $<2$ & $<1$ & $<0.5$ & $<0.5$ & $<0.5$ & $<0.5$ & $<1$ \\
\hline High Lakes 9 & $<0.5$ & $<0.5$ & $<0.5$ & $<0.5$ & $<0.5$ & $<2$ & $<2$ & $<1$ & $<0.5$ & $<0.5$ & $<0.5$ & $<0.5$ & $<1$ \\
\hline \multicolumn{14}{|c|}{ Pine Forest Road Transect } \\
\hline Pine 1 & $<0.5$ & $<0.5$ & $<0.5$ & $<0.5$ & $\mathrm{M}$ & $<2$ & $<2$ & $<1$ & $<0.5$ & $<0.5$ & $<0.5$ & $<0.5$ & $<1$ \\
\hline Pine 2 & $<0.5$ & $<0.5$ & $<0.5$ & $<0.5$ & $<0.5$ & $<2$ & $<2$ & $<1$ & $<0.5$ & $<0.5$ & $<0.5$ & $<0.5$ & $<1$ \\
\hline Pine 3 & $<0.5$ & $<0.5$ & $<0.5$ & $<0.5$ & $<0.5$ & $<2$ & $<2$ & $<1$ & $<0.5$ & $<0.5$ & $<0.5$ & $<0.5$ & $<1$ \\
\hline Pine 4 & $<0.5$ & $<0.5$ & $<0.5$ & $<0.5$ & $<0.5$ & $<2$ & $<2$ & M & $<0.5$ & $<0.5$ & $<0.5$ & $<0.5$ & $<1$ \\
\hline Pine 5 & $<0.5$ & $<0.5$ & $<0.5$ & $<0.5$ & $<0.5$ & $<2$ & $<2$ & $<1$ & $<0.5$ & $<0.5$ & $<0.5$ & $<0.5$ & $<1$ \\
\hline Pine 6 & $<0.5$ & $<0.5$ & $<0.5$ & $<0.5$ & $<0.5$ & $<2$ & $<2$ & $<1$ & $<0.5$ & $<0.5$ & $<0.5$ & $<0.5$ & $<1$ \\
\hline Pine 7 & $<0.5$ & $<0.5$ & $<0.5$ & $<0.5$ & $<0.5$ & $<2$ & $<2$ & $<1$ & $<0.5$ & $<0.5$ & $<0.5$ & $<0.5$ & $<1$ \\
\hline Pine 8 & $<0.5$ & $<0.5$ & $<0.5$ & $<0.5$ & $<0.5$ & $<2$ & $<2$ & $<1$ & $<0.5$ & $<0.5$ & $<0.5$ & $<0.5$ & $<1$ \\
\hline Pine 9 & $<0.5$ & $<0.5$ & $<0.5$ & $<0.5$ & $<0.5$ & $<2$ & $<2$ & $<1$ & $<0.5$ & $<0.5$ & $<0.5$ & $<0.5$ & $<1$ \\
\hline Pine 10 & $<0.5$ & $<0.5$ & $<0.5$ & $<0.5$ & $<0.5$ & $<2$ & $<2$ & $<1$ & $<0.5$ & $<0.5$ & $<0.5$ & $<0.5$ & $<1$ \\
\hline Pine 11 & $<0.5$ & $<0.5$ & $<0.5$ & $<0.5$ & $<0.5$ & $<2$ & $<2$ & $<1$ & $<0.5$ & $<0.5$ & $<0.5$ & $<0.5$ & $<1$ \\
\hline
\end{tabular}


Table 7. Concentrations of organic wastewater compounds in ground water along transects, La Pine, Oregon, 2003.—Continued

[Benzophenone concentrations are reported in this table as they were reported by the laboratory, but are interpreted in the report relative to a project censoring level of 1 microgram per liter; see discussion of organic wastewater compounds in the results and discussion section of the text for explanation of this project censoring level; Date as year, month, day (YYYYMMDD); Time in hours and minutes, military; Chloride in milligrams per liter; Nitrite-plus-nitrate in milligrams N per liter; D.O., dissolved oxygen, in milligrams per liter; Specific conductance in microsiemens per centimeter at 25 degrees Celsius; Temperature in degrees Celsius; Organic wastewater compounds in micrograms per liter; surrogate recoveries in percent; parameter codes for organic wastewater compound names: Pxxxx; "E", estimated; "<", less than; "M", presence verified, not quantified; —, no data]

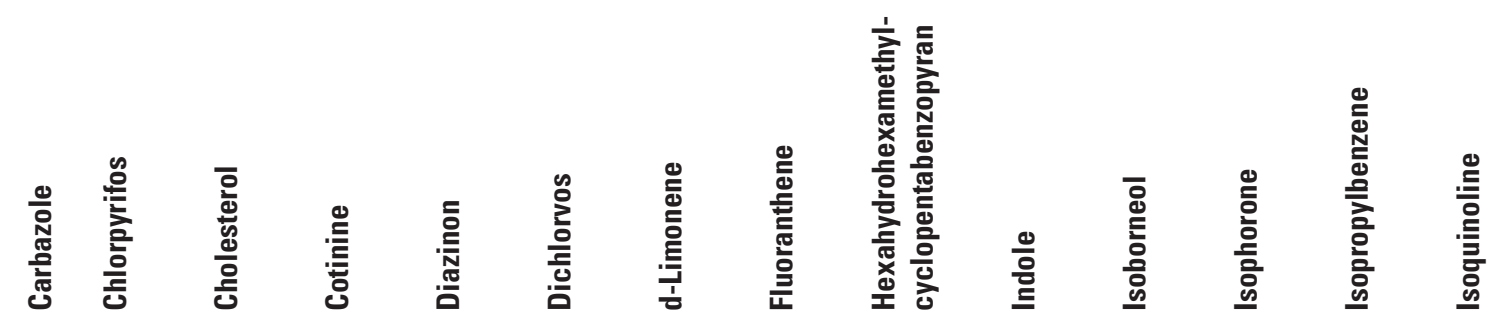

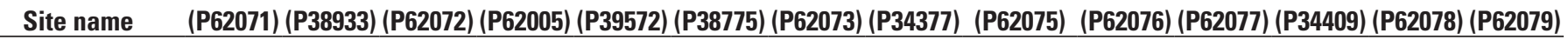

\begin{tabular}{|c|c|c|c|c|c|c|c|c|c|c|c|c|c|c|}
\hline \multicolumn{15}{|c|}{ Senior Center Transect } \\
\hline Senior Septic Tank & $<0.5$ & $<0.5$ & E5 & 3 & $<0.5$ & $<1$ & E0.4 & $<0.5$ & E0.1 & 13 & 7.5 & $<0.5$ & $<0.5$ & $<0.5$ \\
\hline Senior 1 & $<0.5$ & $<0.5$ & $<2$ & $<1$ & $<0.5$ & $<1$ & $<0.5$ & $<0.5$ & M & $<0.5$ & $<0.5$ & $<0.5$ & $<0.5$ & $<0.5$ \\
\hline Senior 2 & $<0.5$ & $<0.5$ & $<2$ & $<1$ & $<0.5$ & $<1$ & $<0.5$ & $<0.5$ & $<0.5$ & M & $<0.5$ & $<0.5$ & $<0.5$ & $<0.5$ \\
\hline Senior 3 & $<0.5$ & $<0.5$ & $<2$ & $<1$ & $<0.5$ & $<1$ & $<0.5$ & $<0.5$ & $<0.5$ & $<0.5$ & $<0.5$ & $<0.5$ & $<0.5$ & $<0.5$ \\
\hline Senior 4 & $<0.5$ & $<0.5$ & $<2$ & $<1$ & $<0.5$ & $<1$ & $<0.5$ & $<0.5$ & $<0.5$ & $<0.5$ & $<0.5$ & $<0.5$ & $<0.5$ & $<0.5$ \\
\hline Senior 5 & $<0.5$ & $<0.5$ & $<2$ & $<1$ & $<0.5$ & $<1$ & $<0.5$ & $<0.5$ & $<0.5$ & $<0.5$ & $<0.5$ & $<0.5$ & $<0.5$ & $<0.5$ \\
\hline Senior 6 & $<0.5$ & $<0.5$ & $<2$ & $<1$ & $<0.5$ & $<1$ & $<0.5$ & $<0.5$ & $<0.5$ & $<0.5$ & $<0.5$ & $<0.5$ & $<0.5$ & $<0.5$ \\
\hline Senior 7 & $<0.5$ & $<0.5$ & $<2$ & $<1$ & $<0.5$ & $<1$ & $<0.5$ & $<0.5$ & $<0.5$ & $<0.5$ & $<0.5$ & $<0.5$ & $<0.5$ & $<0.5$ \\
\hline Senior 8 & $<0.5$ & $<0.5$ & $<2$ & $<1$ & $<0.5$ & $<1$ & $<0.5$ & $<0.5$ & $<0.5$ & $<0.5$ & $<0.5$ & $<0.5$ & $<0.5$ & $<0.5$ \\
\hline Senior 9 & $<0.5$ & $<0.5$ & $<2$ & $<1$ & $<0.5$ & $<1$ & $<0.5$ & $<0.5$ & $<0.5$ & $<0.5$ & $<0.5$ & $<0.5$ & $<0.5$ & $<0.5$ \\
\hline Senior 10 & $<0.5$ & $<0.5$ & $<2$ & $<1$ & $<0.5$ & $<1$ & $<0.5$ & $<0.5$ & $<0.5$ & $<0.5$ & $<0.5$ & $<0.5$ & $<0.5$ & $<0.5$ \\
\hline$\underline{\text { Senior } 11}$ & $<0.5$ & $<0.5$ & $<2$ & $<1$ & $<0.5$ & $<1$ & $<0.5$ & $<0.5$ & $<0.5$ & $<0.5$ & $<0.5$ & $<0.5$ & $<0.5$ & $<0.5$ \\
\hline \multicolumn{15}{|c|}{ High Lakes Church Transect } \\
\hline High Lakes 1 & $<0.5$ & $<0.5$ & $<2$ & $<1$ & $<0.5$ & $<1$ & $<0.5$ & $<0.5$ & $<0.5$ & $<0.5$ & $<0.5$ & $<0.5$ & $<0.5$ & $<0.5$ \\
\hline High Lakes 2 & $<0.5$ & $<0.5$ & $<2$ & $<1$ & $<0.5$ & $<1$ & $<0.5$ & $<0.5$ & $<0.5$ & $<0.5$ & $<0.5$ & $<0.5$ & $<0.5$ & $<0.5$ \\
\hline High Lakes 3 & $<0.5$ & $<0.5$ & $<2$ & $<1$ & $<0.5$ & $<1$ & $<0.5$ & $<0.5$ & $<0.5$ & $<0.5$ & $<0.5$ & $<0.5$ & $<0.5$ & $<0.5$ \\
\hline High Lakes 4 & $<0.5$ & $<0.5$ & $<2$ & $<1$ & $<0.5$ & $<1$ & $<0.5$ & $<0.5$ & $<0.5$ & $<0.5$ & $<0.5$ & $<0.5$ & $<0.5$ & $<0.5$ \\
\hline High Lakes 5 & $<0.5$ & $<0.5$ & $<2$ & $<1$ & $<0.5$ & $<1$ & $<0.5$ & $<0.5$ & $<0.5$ & $<0.5$ & $<0.5$ & $<0.5$ & $<0.5$ & $<0.5$ \\
\hline High Lakes 6 & $<0.5$ & $<0.5$ & $<2$ & $<1$ & $<0.5$ & $<1$ & $<0.5$ & $<0.5$ & $<0.5$ & $<0.5$ & $<0.5$ & $<0.5$ & $<0.5$ & $<0.5$ \\
\hline High Lakes 7 & $<0.5$ & $<0.5$ & $<2$ & $<1$ & $<0.5$ & $<1$ & $<0.5$ & $<0.5$ & $<0.5$ & $<0.5$ & $<0.5$ & $<0.5$ & $<0.5$ & $<0.5$ \\
\hline High Lakes 8 & $<0.5$ & $<0.5$ & $<2$ & $<1$ & $<0.5$ & $<1$ & $<0.5$ & $<0.5$ & $<0.5$ & $<0.5$ & $<0.5$ & $<0.5$ & $<0.5$ & $<0.5$ \\
\hline High Lakes 9 & $<0.5$ & $<0.5$ & $<2$ & $<1$ & $<0.5$ & $<1$ & $<0.5$ & $<0.5$ & $<0.5$ & $<0.5$ & $<0.5$ & $<0.5$ & $<0.5$ & $<0.5$ \\
\hline \multicolumn{15}{|c|}{ Pine Forest Road Transect } \\
\hline Pine 1 & $<0.5$ & $<0.5$ & $<2$ & $<1$ & $<0.5$ & $<1$ & $<0.5$ & $<0.5$ & $<0.5$ & $<0.5$ & $<0.5$ & $<0.5$ & $<0.5$ & $<0.5$ \\
\hline Pine 2 & $<0.5$ & $<0.5$ & $<2$ & $<1$ & $<0.5$ & $<1$ & $<0.5$ & $<0.5$ & $<0.5$ & $<0.5$ & $<0.5$ & $<0.5$ & $<0.5$ & $<0.5$ \\
\hline Pine 3 & $<0.5$ & $<0.5$ & $<2$ & $<1$ & $<0.5$ & $<1$ & $<0.5$ & $<0.5$ & $<0.5$ & $<0.5$ & $<0.5$ & $<0.5$ & $<0.5$ & $<0.5$ \\
\hline Pine 4 & $<0.5$ & $<0.5$ & $<2$ & $<1$ & $<0.5$ & $<1$ & $<0.5$ & $<0.5$ & $<0.5$ & $<0.5$ & $<0.5$ & $<0.5$ & $<0.5$ & $<0.5$ \\
\hline Pine 5 & $<0.5$ & $<0.5$ & $<2$ & $<1$ & $<0.5$ & $<1$ & $<0.5$ & $<0.5$ & $<0.5$ & $<0.5$ & $<0.5$ & $<0.5$ & $<0.5$ & $<0.5$ \\
\hline Pine 6 & $<0.5$ & $<0.5$ & $<2$ & $<1$ & $<0.5$ & $<1$ & $\mathrm{M}$ & $<0.5$ & $<0.5$ & $<0.5$ & $<0.5$ & $<0.5$ & $<0.5$ & $<0.5$ \\
\hline Pine 7 & $<0.5$ & $<0.5$ & $<2$ & $<1$ & $<0.5$ & $<1$ & $<0.5$ & $<0.5$ & $<0.5$ & $<0.5$ & $<0.5$ & $<0.5$ & $<0.5$ & $<0.5$ \\
\hline Pine 8 & $<0.5$ & $<0.5$ & $<2$ & $<1$ & $<0.5$ & $<1$ & $<0.5$ & $<0.5$ & $<0.5$ & $<0.5$ & $<0.5$ & $<0.5$ & $<0.5$ & $<0.5$ \\
\hline Pine 9 & $<0.5$ & $<0.5$ & $<2$ & $<1$ & $<0.5$ & $<1$ & $<0.5$ & $<0.5$ & $<0.5$ & $<0.5$ & $<0.5$ & $<0.5$ & $<0.5$ & $<0.5$ \\
\hline Pine 10 & $<0.5$ & $<0.5$ & $<2$ & $<1$ & $<0.5$ & $<1$ & $<0.5$ & $<0.5$ & $<0.5$ & $<0.5$ & $<0.5$ & $<0.5$ & $<0.5$ & $<0.5$ \\
\hline Pine 11 & $<0.5$ & $<0.5$ & $<2$ & $<1$ & $<0.5$ & $<1$ & $<0.5$ & $<0.5$ & $<0.5$ & $<0.5$ & $<0.5$ & $<0.5$ & $<0.5$ & $<0.5$ \\
\hline
\end{tabular}


Table 7. Concentrations of organic wastewater compounds in ground water along transects, La Pine, Oregon, 2003.—Continued

[Benzophenone concentrations are reported in this table as they were reported by the laboratory, but are interpreted in the report relative to a project censoring level of 1 microgram per liter; see discussion of organic wastewater compounds in the results and discussion section of the text for explanation of this project censoring level; Date as year, month, day (YYYYMMDD); Time in hours and minutes, military; Chloride in milligrams per liter; Nitrite-plus-nitrate in milligrams N per liter; D.O., dissolved oxygen, in milligrams per liter; Specific conductance in microsiemens per centimeter at 25 degrees Celsius; Temperature in degrees Celsius; Organic wastewater compounds in micrograms per liter; surrogate recoveries in percent; parameter codes for organic wastewater compound names: Pxxxx; "E", estimated; "<", less than; "M", presence verified, not quantified; - , no data]

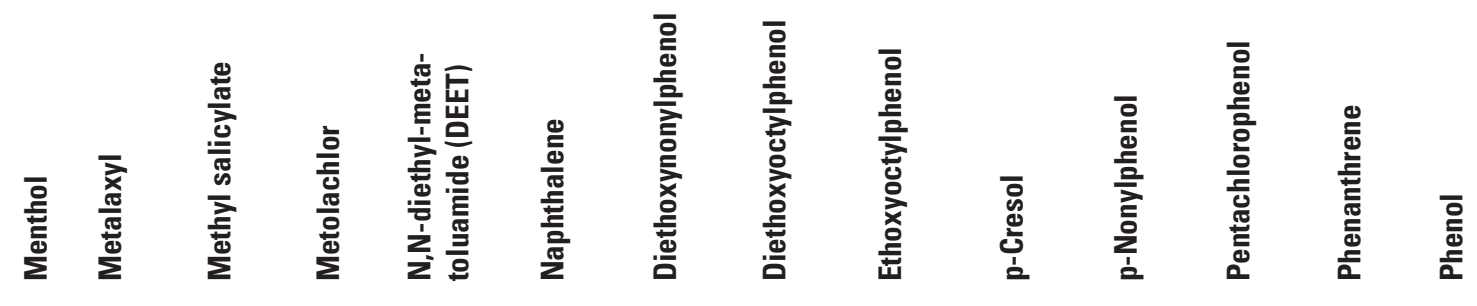

Site name

(P62080) (P50359) (P62081) (P39415) (P62082) (P34443) (P62083) (P61705) (P61706) (P62084) (P62085) (P34459) (P34462)P34466)

Senior Center Transect

\begin{tabular}{|c|c|c|c|c|c|c|c|c|c|c|c|c|c|c|}
\hline Senior Septic Tank & 22 & $<0.5$ & 1.7 & $<0.5$ & $<0.5$ & $<0.5$ & E13 & $<1$ & E1 & 190 & E7 & $<2$ & $<0.5$ & 22 \\
\hline Senior 1 & $<0.5$ & $<0.5$ & $<0.5$ & $<0.5$ & M & $<0.5$ & $<5$ & $<1$ & $<1$ & M & $<6$ & $<2$ & $<0.5$ & $<4$ \\
\hline Senior 2 & M & $<0.5$ & $<0.5$ & $<0.5$ & $<0.5$ & $<0.5$ & $<5$ & $<1$ & $<1$ & M & $<6$ & $<2$ & $<0.5$ & $<4$ \\
\hline Senior 3 & $<0.5$ & $<0.5$ & $<0.5$ & $<0.5$ & $<0.5$ & $<0.5$ & $<5$ & $<1$ & $<1$ & M & $<6$ & $<2$ & $<0.5$ & $<4$ \\
\hline Senior 4 & $<0.5$ & $<0.5$ & $<0.5$ & $<0.5$ & $<0.5$ & $<0.5$ & $<5$ & $<1$ & $<1$ & $<1$ & $<6$ & $<2$ & $<0.5$ & $<4$ \\
\hline Senior 5 & $<0.5$ & $<0.5$ & $<0.5$ & $<0.5$ & $<0.5$ & $<0.5$ & $<5$ & $<1$ & $<1$ & $<1$ & $<6$ & $<2$ & $<0.5$ & $<4$ \\
\hline Senior 6 & M & $<0.5$ & $<0.5$ & $<0.5$ & $<0.5$ & $<0.5$ & $\mathrm{M}$ & $<1$ & $<1$ & $<1$ & $<6$ & $<2$ & $<0.5$ & $<4$ \\
\hline Senior 7 & M & $<0.5$ & $<0.5$ & $<0.5$ & $<0.5$ & $<0.5$ & $<5$ & $<1$ & $<1$ & $<1$ & $<6$ & $<2$ & $<0.5$ & $<4$ \\
\hline Senior 8 & $<0.5$ & $<0.5$ & $<0.5$ & $<0.5$ & $<0.5$ & $<0.5$ & $<5$ & $<1$ & $<1$ & $<1$ & $<6$ & $<2$ & $<0.5$ & $<4$ \\
\hline Senior 9 & $<0.5$ & $<0.5$ & $<0.5$ & $<0.5$ & $<0.5$ & $<0.5$ & $<5$ & $<1$ & $<1$ & $<1$ & $<6$ & $<2$ & $<0.5$ & $<4$ \\
\hline Senior 10 & $<0.5$ & $<0.5$ & $<0.5$ & $<0.5$ & $<0.5$ & $<0.5$ & $<5$ & $<1$ & $<1$ & M & $<6$ & $<2$ & $<0.5$ & $<4$ \\
\hline$\underline{\text { Senior } 11}$ & $<0.5$ & $<0.5$ & $<0.5$ & $<0.5$ & $<0.5$ & $<0.5$ & $<5$ & $<1$ & $<1$ & $<1$ & $<6$ & $<2$ & $<0.5$ & $<4$ \\
\hline
\end{tabular}

High Lakes Church Transect

\begin{tabular}{|c|c|c|c|c|c|c|c|c|c|c|c|c|c|c|}
\hline High Lakes 1 & $<0.5$ & $<0.5$ & $<0.5$ & $<0.5$ & $<0.5$ & $<0.5$ & $<5$ & $<1$ & $<1$ & $<1$ & $<6$ & $<2$ & $<0.5$ & $<4$ \\
\hline High Lakes 2 & $<0.5$ & $<0.5$ & $<0.5$ & $<0.5$ & E0.1 & $<0.5$ & $<5$ & $<1$ & $<1$ & $<1$ & $<6$ & $<2$ & $<0.5$ & $<4$ \\
\hline High Lakes 3 & $<0.5$ & $<0.5$ & $<0.5$ & $<0.5$ & $<0.5$ & $<0.5$ & $<5$ & $<1$ & $<1$ & $<1$ & $<6$ & $<2$ & $<0.5$ & $<4$ \\
\hline High Lakes 4 & $<0.5$ & $<0.5$ & $<0.5$ & $<0.5$ & $<0.5$ & $<0.5$ & $<5$ & $<1$ & $<1$ & $<1$ & $<6$ & $<2$ & $<0.5$ & $<4$ \\
\hline High Lakes 5 & $<0.5$ & $<0.5$ & $<0.5$ & $<0.5$ & E0.1 & $<0.5$ & $<5$ & $<1$ & $<1$ & $<1$ & $<6$ & $<2$ & $<0.5$ & $<4$ \\
\hline High Lakes 6 & $<0.5$ & $<0.5$ & $<0.5$ & $<0.5$ & $<0.5$ & $<0.5$ & $<5$ & $<1$ & $<1$ & $<1$ & $<6$ & $<2$ & $<0.5$ & $<4$ \\
\hline High Lakes 7 & $<0.5$ & $<0.5$ & $<0.5$ & $<0.5$ & $<0.5$ & $<0.5$ & $<5$ & $<1$ & $<1$ & $<1$ & $<6$ & $<2$ & $<0.5$ & $<4$ \\
\hline High Lakes 8 & $<0.5$ & $<0.5$ & $<0.5$ & $<0.5$ & $<0.5$ & $<0.5$ & $<5$ & $<1$ & $<1$ & $<1$ & $<6$ & $<2$ & $<0.5$ & $<4$ \\
\hline High Lakes 9 & $<0.5$ & $<0.5$ & $<0.5$ & $<0.5$ & $<0.5$ & $<0.5$ & $<5$ & $<1$ & $<1$ & $<1$ & $<6$ & $<2$ & $<0.5$ & $<4$ \\
\hline \multicolumn{15}{|c|}{ Pine Forest Road Transect } \\
\hline Pine 1 & $<0.5$ & $<0.5$ & $<0.5$ & $<0.5$ & $\mathrm{M}$ & $<0.5$ & $<5$ & $<1$ & $<1$ & $<1$ & $<6$ & $<2$ & $<0.5$ & $<4$ \\
\hline Pine 2 & $<0.5$ & $<0.5$ & $<0.5$ & $<0.5$ & $<0.5$ & $<0.5$ & $<5$ & $<1$ & $<1$ & $<1$ & $<6$ & $<2$ & $<0.5$ & $<4$ \\
\hline Pine 3 & $<0.5$ & $<0.5$ & $<0.5$ & $<0.5$ & $<0.5$ & $<0.5$ & $<5$ & $<1$ & $<1$ & $<1$ & $<6$ & $<2$ & $<0.5$ & $<4$ \\
\hline Pine 4 & $<0.5$ & $<0.5$ & $<0.5$ & $<0.5$ & $<0.5$ & $<0.5$ & $<5$ & $<1$ & $<1$ & $<1$ & $<6$ & $<2$ & $<0.5$ & $<4$ \\
\hline Pine 5 & $<0.5$ & $<0.5$ & $<0.5$ & $<0.5$ & $<0.5$ & $<0.5$ & $<5$ & $<1$ & $<1$ & $<1$ & $<6$ & $<2$ & $<0.5$ & $<4$ \\
\hline Pine 6 & $<0.5$ & $<0.5$ & $<0.5$ & $<0.5$ & $<0.5$ & $<0.5$ & $<5$ & $<1$ & $<1$ & $<1$ & $<6$ & $<2$ & $<0.5$ & $<4$ \\
\hline Pine 7 & $<0.5$ & $<0.5$ & $<0.5$ & $<0.5$ & 0.8 & $<0.5$ & $<5$ & $<1$ & $<1$ & $<1$ & $<6$ & $<2$ & $<0.5$ & $<4$ \\
\hline Pine 8 & $<0.5$ & $<0.5$ & $<0.5$ & $<0.5$ & E0.4 & $<0.5$ & $<5$ & $<1$ & $<1$ & $<1$ & $<6$ & $<2$ & $<0.5$ & $<4$ \\
\hline Pine 9 & $<0.5$ & $<0.5$ & $<0.5$ & $<0.5$ & E0.3 & $<0.5$ & $<5$ & $<1$ & $<1$ & $<1$ & $<6$ & $<2$ & $<0.5$ & $<4$ \\
\hline Pine 10 & $<0.5$ & $<0.5$ & $<0.5$ & $<0.5$ & $<0.5$ & $<0.5$ & $<5$ & $<1$ & $<1$ & $<1$ & $<6$ & $<2$ & $<0.5$ & $<4$ \\
\hline Pine 11 & $<0.5$ & $<0.5$ & $<0.5$ & $<0.5$ & $<0.5$ & $<0.5$ & $<5$ & $<1$ & $<1$ & $<1$ & $<6$ & $<2$ & $<0.5$ & $<4$ \\
\hline
\end{tabular}


Table 7. Concentrations of organic wastewater compounds in ground water along transects, La Pine, Oregon, 2003.-Continued

[Benzophenone concentrations are reported in this table as they were reported by the laboratory, but are interpreted in the report relative to a project censoring level of 1 microgram per liter; see discussion of organic wastewater compounds in the results and discussion section of the text for explanation of this project censoring level; Date as year, month, day (YYYYMMDD); Time in hours and minutes, military; Chloride in milligrams per liter; Nitrite-plus-nitrate in milligrams N per liter; D.O., dissolved oxygen, in milligrams per liter; Specific conductance in microsiemens per centimeter at 25 degrees Celsius; Temperature in degrees Celsius; Organic wastewater compounds in micrograms per liter; surrogate recoveries in percent; parameter codes for organic wastewater compound names: Pxxxx; "E”, estimated; “<”, less than; "M", presence verified, not quantified; —, no data]

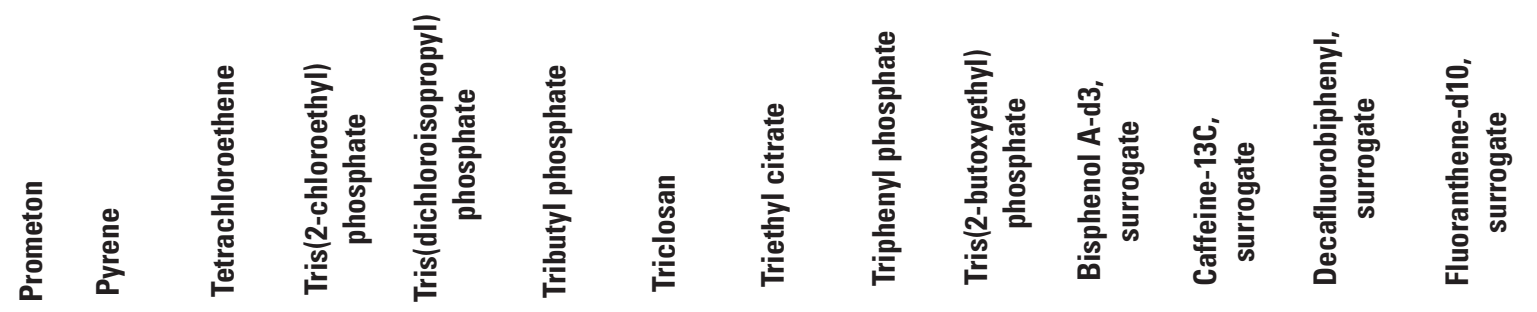

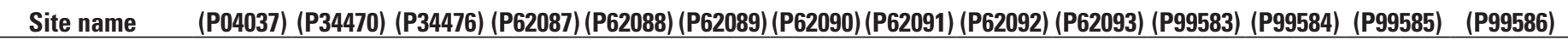

\begin{tabular}{|c|c|c|c|c|c|c|c|c|c|c|c|c|c|c|}
\hline \multicolumn{15}{|c|}{ Senior Center Transect } \\
\hline Senior Septic Tank & $<0.5$ & $<0.5$ & $<0.5$ & E0.2 & $<0.5$ & $<0.5$ & 3 & E0.4 & E0.1 & 8.9 & 115 & 105 & 76.3 & 96.6 \\
\hline Senior 1 & $<0.5$ & $<0.5$ & $<0.5$ & $<0.5$ & $<0.5$ & E0.1 & $<1$ & $<0.5$ & $<0.5$ & $<0.5$ & 66.7 & 108 & 83.3 & 100 \\
\hline Senior 2 & $<0.5$ & $<0.5$ & $<0.5$ & $<0.5$ & $<0.5$ & $<0.5$ & M & $<0.5$ & $<0.5$ & $<0.5$ & 80.0 & 120 & 88.0 & 104 \\
\hline Senior 3 & $<0.5$ & $<0.5$ & M & $<0.5$ & $<0.5$ & $<0.5$ & $<1$ & $<0.5$ & $<0.5$ & $<0.5$ & 96.0 & 104 & 88.0 & 96.0 \\
\hline Senior 4 & $<0.5$ & $<0.5$ & $<0.5$ & $<0.5$ & $<0.5$ & $<0.5$ & $<1$ & $<0.5$ & $<0.5$ & $<0.5$ & 10.4 & 130 & 87.0 & 113 \\
\hline Senior 5 & $<0.5$ & $<0.5$ & $\mathrm{M}$ & $<0.5$ & $<0.5$ & $<0.5$ & $<1$ & $<0.5$ & $<0.5$ & $<0.5$ & 95.7 & 126 & 87.0 & 109 \\
\hline Senior 6 & $<0.5$ & $<0.5$ & $<0.5$ & $<0.5$ & $<0.5$ & $<0.5$ & $<1$ & $<0.5$ & $<0.5$ & $<0.5$ & 95.5 & 123 & 90.9 & 109 \\
\hline Senior 7 & $<0.5$ & $<0.5$ & $<0.5$ & $<0.5$ & $<0.5$ & $<0.5$ & $<1$ & $<0.5$ & $<0.5$ & $<0.5$ & 47.8 & 130 & 91.3 & 113 \\
\hline Senior 8 & $<0.5$ & $<0.5$ & E0.1 & $<0.5$ & $<0.5$ & $<0.5$ & $<1$ & $<0.5$ & $<0.5$ & $<0.5$ & 100 & 122 & 87.0 & 104 \\
\hline Senior 9 & $<0.5$ & $<0.5$ & $<0.5$ & $<0.5$ & $<0.5$ & $<0.5$ & $<1$ & $<0.5$ & $<0.5$ & $<0.5$ & 6.5 & 130 & 87.0 & 113 \\
\hline Senior 10 & $<0.5$ & $<0.5$ & $<0.5$ & $<0.5$ & $<0.5$ & $<0.5$ & $<1$ & $<0.5$ & $<0.5$ & $<0.5$ & 44.1 & 123 & 86.4 & 105 \\
\hline$\underline{\text { Senior } 11}$ & $<0.5$ & $<0.5$ & $<0.5$ & $<0.5$ & $<0.5$ & $<0.5$ & $<1$ & $<0.5$ & $<0.5$ & $<0.5$ & 60.9 & 117 & 104 & 122 \\
\hline \multicolumn{15}{|c|}{ High Lakes Church Transect } \\
\hline High Lakes 1 & $<0.5$ & $<0.5$ & $<0.5$ & $<0.5$ & $<0.5$ & $<0.5$ & $<1$ & $<0.5$ & $<0.5$ & $<0.5$ & 58.3 & 87.5 & 58.3 & 91.7 \\
\hline High Lakes 2 & $<0.5$ & $<0.5$ & $<0.5$ & $<0.5$ & $<0.5$ & $<0.5$ & $<1$ & $<0.5$ & $<0.5$ & $<0.5$ & 91.7 & 100 & 70.8 & 100 \\
\hline High Lakes 3 & $<0.5$ & $<0.5$ & $<0.5$ & $<0.5$ & $<0.5$ & $<0.5$ & $<1$ & $<0.5$ & $<0.5$ & $<0.5$ & 56.0 & 80.0 & 60.0 & 88.0 \\
\hline High Lakes 4 & $<0.5$ & $<0.5$ & $<0.5$ & $<0.5$ & $<0.5$ & $<0.5$ & $<1$ & $<0.5$ & $<0.5$ & $<0.5$ & 66.7 & 91.7 & 66.7 & 100 \\
\hline High Lakes 5 & $<0.5$ & $<0.5$ & $<0.5$ & $<0.5$ & $<0.5$ & $<0.5$ & $<1$ & $<0.5$ & $<0.5$ & $<0.5$ & 70.8 & 95.8 & 58.3 & 95.8 \\
\hline High Lakes 6 & $<0.5$ & $<0.5$ & $<0.5$ & $<0.5$ & $<0.5$ & $<0.5$ & $<1$ & $<0.5$ & $<0.5$ & $<0.5$ & 87.0 & 91.3 & 56.5 & 100 \\
\hline High Lakes 7 & $<0.5$ & $<0.5$ & $<0.5$ & $<0.5$ & $<0.5$ & $<0.5$ & $<1$ & $<0.5$ & $<0.5$ & $<0.5$ & 82.6 & 91.3 & 56.5 & 100 \\
\hline High Lakes 8 & $<0.5$ & $<0.5$ & $<0.5$ & $<0.5$ & $<0.5$ & $<0.5$ & $<1$ & $<0.5$ & $<0.5$ & $<0.5$ & 78.3 & 100 & 60.9 & 100 \\
\hline High Lakes 9 & $<0.5$ & $<0.5$ & $<0.5$ & $<0.5$ & $<0.5$ & $<0.5$ & $<1$ & $<0.5$ & $<0.5$ & $<0.5$ & 95.7 & 100 & 69.6 & 95.7 \\
\hline \multicolumn{15}{|c|}{ Pine Forest Road Transect } \\
\hline Pine 1 & $<0.5$ & $<0.5$ & $<0.5$ & E0.1 & $<0.5$ & $<0.5$ & $<1$ & $<0.5$ & $<0.5$ & $<0.5$ & 73.9 & 87.0 & 78.3 & 82.6 \\
\hline Pine 2 & $<0.5$ & $<0.5$ & $<0.5$ & $<0.5$ & $<0.5$ & $<0.5$ & $<1$ & $<0.5$ & $<0.5$ & $<0.5$ & 69.6 & 91.3 & 69.6 & 87.0 \\
\hline Pine 3 & $<0.5$ & $<0.5$ & $<0.5$ & $<0.5$ & $<0.5$ & $<0.5$ & $<1$ & $<0.5$ & $<0.5$ & $<0.5$ & 65.2 & 91.3 & 78.3 & 87.0 \\
\hline Pine 4 & $<0.5$ & $<0.5$ & $<0.5$ & $<0.5$ & $<0.5$ & $<0.5$ & $<1$ & $<0.5$ & $<0.5$ & $<0.5$ & 66.7 & 91.7 & 66.7 & 91.7 \\
\hline Pine 5 & $<0.5$ & $<0.5$ & $<0.5$ & $<0.5$ & $<0.5$ & $<0.5$ & $<1$ & $<0.5$ & $<0.5$ & $<0.5$ & 78.3 & 91.3 & 56.5 & 100 \\
\hline Pine 6 & $<0.5$ & $<0.5$ & $<0.5$ & $<0.5$ & $<0.5$ & $<0.5$ & $<1$ & $<0.5$ & $<0.5$ & $<0.5$ & 86.4 & 95.5 & 63.6 & 95.5 \\
\hline Pine 7 & $<0.5$ & $<0.5$ & $<0.5$ & $<0.5$ & $<0.5$ & $<0.5$ & $<1$ & $<0.5$ & $<0.5$ & $<0.5$ & 69.6 & 95.7 & 69.6 & 100 \\
\hline Pine 8 & $<0.5$ & $<0.5$ & $<0.5$ & $<0.5$ & $<0.5$ & $<0.5$ & $<1$ & $<0.5$ & $<0.5$ & $<0.5$ & 79.2 & 91.7 & 66.7 & 100 \\
\hline Pine 9 & $<0.5$ & $<0.5$ & $<0.5$ & $<0.5$ & $<0.5$ & $<0.5$ & $<1$ & $<0.5$ & $<0.5$ & $<0.5$ & 82.6 & 91.3 & 73.9 & 95.7 \\
\hline Pine 10 & $<0.5$ & $<0.5$ & $<0.5$ & $<0.5$ & $<0.5$ & $<0.5$ & $<1$ & $<0.5$ & $<0.5$ & $<0.5$ & 75.0 & 83.3 & 87.5 & 91.7 \\
\hline Pine 11 & $<0.5$ & $<0.5$ & $<0.5$ & E0.1 & $<0.5$ & $<0.5$ & $<1$ & $<0.5$ & $<0.5$ & $<0.5$ & 73.9 & 87.0 & 87.0 & 91.3 \\
\hline
\end{tabular}


This sample contained benzophenone, at a concentration of $\mathrm{E}$ $0.1 \mu \mathrm{g} / \mathrm{L}$, but no other organic wastewater compounds were detected. One approach to interpretation of the detection of benzophenone in this environmental blank would be to censor benzophenone concentrations at $1 \mu \mathrm{g} / \mathrm{L}$. The above definition of an environmental blank is an arbitrary one, and it is recognized that other interpretations are possible. Thus, for the case of benzophenone, uncensored benzophenone concentrations for environmental samples are shown in tables 5 and 6 as reported by the NWQL, but interpretations in this report are made relative to a project censoring level of $1 \mu \mathrm{g} / \mathrm{L}$.

\section{Organic Wastewater Compounds in Onsite Wastewater}

Samples of onsite wastewater (20 from the innovative system network, and 1 from the Senior transect) were analyzed for 63 organic wastewater compounds. The number of detections of organic wastewater compounds in the Senior transect onsite wastewater sample (29) was similar to the mean (26) and median (28) number of detections in the 20 samples of the single-family onsite wastewater treatment systems in the innovative system network. Also, the magnitude of the concentrations of organic wastewater compounds in the transect onsite wastewater sample was similar to that observed in the 20 samples of single-family onsite wastewater. On this basis, the results of the Senior transect and innovative system network onsite wastewater samples were evaluated as a group for interpretive purposes.

Of the 63 organic wastewater compounds in the analytical suite, 45 were detected in onsite wastewater, at concentrations ranging from less than $1 \mu \mathrm{g} / \mathrm{L}$ to $1,300 \mu \mathrm{g} / \mathrm{L}$ (p-cresol). Caffeine was detected at up to $320 \mu \mathrm{g} / \mathrm{L}$. Of the 45 organic wastewater compounds that were detected in onsite wastewater, 14 were detected in more than 90 percent of samples; these frequently detected organic wastewater compounds are listed in table 8, along with concentration ranges, medians, and means. Eight of these 14 frequently detected organic wastewater compounds [3-beta-coprostanol, caffeine, cholesterol, N,N-diethyl-meta-toluamide (DEET), diethoxynonylphenol, p-cresol, tris(2-chloroethyl) phosphate, triphenyl phosphate] were part of a suite of 46 organic wastewater compounds in a national assessment of organic wastewater compound occurrence in streams affected by human, industrial and agricultural wastewater (Kolpin and others, 2002). The eight organic wastewater compounds that were (1) detected in more than 90 percent of onsite wastewater samples in the La Pine study, and (2) were analyzed for in stream samples by Kolpin and others (2002), were detected in the stream samples around the Nation at a mean detection frequency of 46 percent, compared with a mean detection frequency of 19 percent nationally for the other organic wastewater compounds in the analytical suite used by Kolpin and others (2002). The high frequency of occurrence of the eight organic wastewater compounds in human-affected streams (Kolpin and others, 2002) and in wastewater (La Pine study) demonstrates the likely reliability of most of these eight compounds to serve as indicators of the presence of wastewater in most aqueous environments.

\section{Organic Wastewater Compounds in Lysimeter and Drainfield Monitoring Well Samples}

Drainfield monitoring wells downgradient from onsite wastewater treatment systems were sampled and the water analyzed for organic wastewater compounds to provide information on the occurrence of organic wastewater compounds in ground water. Organic wastewater compounds were detected in drainfield monitoring well samples only at low concentrations; all detections were estimated concentrations below LRLs ("E" codes), and most were below $1 \mu \mathrm{g} / \mathrm{L}$. Eight different organic wastewater compounds were detected in drainfield monitoring well samples, each in from one to six samples (table 9). Organic wastewater compound occurrence, although not necessarily concentrations, in drainfield monitoring well samples indicate a relation to onsite wastewater sources. The 10 samples with highest chloride concentrations contained 17 detections of organic wastewater compounds, whereas the 10 samples with lowest chloride concentrations contained only 3 detections of organic wastewater compounds. (Chloride is a useful tracer of onsite wastewater, and is relatively conservative in the environment. The mean chloride concentration from the spring 2003 samples of onsite wastewater was 54 $\mathrm{mg} / \mathrm{L}$.) However, drainfield monitoring well samples indicate poor correlation between ground-water organic wastewater compound concentration and compound concentration at the source. For example, all detections of hexahydrohexamethylcyclopentabenzopyran (HHCB; the most frequently detected organic wastewater compound in drainfield monitoring well samples, detected six times) were equal to E $0.1 \mu \mathrm{g} / \mathrm{L}$, in spite of highly variable HHCB concentrations in septic tank samples. Normalizing septic tank and drainfield monitoring well sample HHCB concentrations to chloride concentrations did not notably improve the relation. (Rounding effects for these low concentrations of HHCB may have obscured correlations; low estimated concentrations are likely not suitable for correlation analysis.)

Lysimeters at five sites were sampled and the water analyzed for organic wastewater compounds. At these sites, a clear pattern of generally decreasing concentrations from onsite wastewater treatment systems to lysimeters to drainfield monitoring wells is evident, with most attenuation occurring between onsite wastewater treatment systems and lysimeters (table 6).

Frequent detection of many organic wastewater compounds in onsite wastewater provides evidence that these compounds may have potential to be used as indicators of human waste in some environments, and concentration data 
Table 8. List of organic wastewater compounds detected in more than 90 percent of 21 onsite wastewater samples, La Pine, Oregon, 2003.

[Selected, possible compound uses or sources, from compilation of Zaugg and others (2002); $\mu \mathrm{g} / \mathrm{L}$, micrograms per liter; "<", less than; "E", estimated; means are calculated assuming that censored values (values less than the minimum reporting level) are equal to zero]

\begin{tabular}{|c|c|c|c|c|c|c|}
\hline Organic wastewater compound & Parameter code & $\begin{array}{l}\text { Selected, possible compound } \\
\text { uses or sources }\end{array}$ & $\begin{array}{l}\text { Minimum } \\
(\mu \mathrm{g} / \mathrm{L})\end{array}$ & $\begin{array}{l}\text { Median } \\
(\mu \mathrm{g} / \mathrm{L})\end{array}$ & $\begin{array}{l}\text { Mean } \\
(\mu \mathrm{g} / \mathrm{L})\end{array}$ & $\begin{array}{l}\text { Maximum } \\
(\mu \mathrm{g} / \mathrm{L})\end{array}$ \\
\hline 3-beta-Coprostanol & 62057 & Carnivore fecal indicator & $<2$ & E11 & 16 & 53 \\
\hline Acetyl-hexamethyl-tetrahydro-naphthalene & 62065 & Musk fragrance & $<0.5$ & E0.5 & 1.2 & 8.4 \\
\hline Caffeine & 50305 & Beverages & E0.4 & 12 & 49 & 320 \\
\hline Indole & 62076 & Fragrance in coffee & $<0.5$ & 14 & 31 & 220 \\
\hline Menthol & 62080 & Cigarettes, cough drops, mouthwash & $<0.5$ & 21 & 26 & 160 \\
\hline N,N-diethyl-meta-toluamide (DEET) & 62082 & Mosquito repellent & $<0.5$ & 0.8 & 4.0 & 52 \\
\hline Diethoxynonylphenol & 62083 & Nonionic detergent metabolite & $<5$ & E15 & E27 & E 130 \\
\hline Triphenyl phosphate & 62092 & Plasticizer, flame retardant & $<0.5$ & E0.4 & E0.4 & 0.9 \\
\hline
\end{tabular}

${ }^{a}$ Cresols also are present in many foods, are components of tobacco smoke, and are components of some disinfectants (Agency for Toxic Substances and Disease Registry, 2004).

from drainfield monitoring wells demonstrate that organic wastewater compounds are present in ground water downgradient from onsite wastewater. However, the consistently low estimated concentrations (below LRLs) of organic wastewater compounds in samples collected from drainfield monitoring wells located within a few feet (range 0 to 19 feet, and median 6 feet; table 2) of drainfield lines indicates that this suite of organic wastewater compounds may be sufficiently hydrophobic and (or) may degrade sufficiently quickly as to have limited usefulness as tracers of onsite wastewater in aquifers. Even the lysimeter samples, which generally contained organic wastewater compounds at greater concentrations than were present in drainfield monitoring well samples, demonstrated that organic wastewater compounds are mostly attenuated after unsaturated-zone transport of 1 foot.

\section{Organic Wastewater Compounds in Transect Samples}

Monitoring wells installed along plumes of onsite wastewater were sampled and the water analyzed for organic wastewater compounds to provide information on organic wastewater compound occurrence from a ground-watertransect framework. Six organic wastewater compounds were detected in transect monitoring well samples, each in one to five samples (table 9). Although transect monitoring well samples generally contained elevated concentrations of chloride (22 samples containing greater than $5 \mathrm{mg} / \mathrm{L}$, and 15 samples containing greater than $10 \mathrm{mg} / \mathrm{L}$; table 7) and nitrate (19 samples containing greater than $3 \mathrm{mg} \mathrm{N} / \mathrm{L}$, and 10 samples containing greater than $10 \mathrm{mg} \mathrm{N} / \mathrm{L}$; table 7), organic wastewater compounds were detected at generally low concentrations. The insect (mosquito) repellent N,N-diethyl-meta-toluamide (DEET) was detected in one sample at $0.8 \mu \mathrm{g} / \mathrm{L}$, but other detections of DEET, and all other organic wastewater compound detections in transect monitoring well samples, were estimated at concentrations below LRLs ("E" codes). (DEET is a commonly detected organic wastewater compound, and is persistent in ground water; Barnes and others, 2004.)

Organic wastewater compounds along the transects did not occur in patterns that would suggest strong correlation between organic wastewater compounds and proximity to onsite wastewater treatment system drainfield lines or to fraction of onsite wastewater in the samples as inferred by chloride concentrations. For example, of the 31 transect wells, 14 were situated along the upgradient edge of onsite wastewater plumes (Senior 1, 2, 3, 4, 5; High 1, 2, 3, 4, 5; Pine 1, 2, 10, 11 ) and the remaining 17 were distributed further downgradient along the plume axes (figs. 2, 3, and 4). The 14 upgradi- 
Table 9. List of organic wastewater compounds detected in ground-water samples from (a) drainfield monitoring wells, innovative on-site wastewater treatment system network, and (b) transect wells, La Pine, Oregon, 2003.

[“--“, not detected; selected, possible compound uses or sources, as compiled by Zaugg and others (2002): tetrachloroethene, solvent; tris (dichloroisopropyl) phosphate, flame retardant; tributyl phosphate, antifoaming agent, flame retardant; other organic wastewater compounds described in table 8; number of samples: drainfield monitoring wells, 20, and transect wells, 31]

\begin{tabular}{lccc}
\hline \multicolumn{1}{c}{ Organic wastewater compound } & Parameter code & $\begin{array}{c}\text { Detection frequency, } \\
\text { drainfield monitoring wells } \\
\text { (percent) }\end{array}$ & $\begin{array}{c}\text { Detection frequency, } \\
\text { transect wells } \\
\text { (percent) }\end{array}$ \\
\hline Acetyl-hexamethyl-tetrahydro-naphthalene & 62065 & 5 & 3 \\
Caffeine & 50305 & -- & 3 \\
Cholesterol & 62072 & 5 & -- \\
Hexahydrohexamethyl-cyclopentabenzopyran & 62075 & 30 & -- \\
N,N-diethyl-meta-toluamide (DEET) & 62082 & 20 & 16 \\
Tetrachloroethene & 34476 & 5 & 3 \\
Tris (2-chloroethyl) phosphate & 62087 & 15 & 6 \\
Tris (dichloroisopropyl) phosphate & 62088 & 10 & -- \\
Tributyl phosphate & 62089 & 10 & 3 \\
\hline
\end{tabular}

ent-edge wells did yield more frequent organic wastewater compound detections (7) than did the 17 downgradient wells (4 organic wastewater compound detections). These occurrence patterns are consistent with expected trends based on attenuation, but they are not striking. Furthermore, evaluating the 14 upgradient-edge wells either as a group, or as 3 groups representing upgradient-edges of 3 transects (both approaches serving to reduce effects of attenuation during transport along flowpaths) yields no strong correlations with chloride concentrations. These observations stand in contrast to observations of relations between nitrate and chloride concentrations in transect samples. A plot of chloride versus nitrate (fig. 5) demonstrates the strong correlation between chloride and nitrate in transect samples (linear correlation $\mathrm{r}^{2}=0.91$ ), and reactive transport of nitrate can be evaluated by comparing nitrate concentrations to chloride concentrations. Organic wastewater compound transport, though, appears to be controlled by different processes than is chloride or nitrate transport. Organic wastewater compound transport cannot be simulated in the same manner as might nitrate transport. One complicating factor is that many analytes in the organic wastewater compound analytical suite may be introduced into aquifers from routes besides percolation of onsite wastewater. However, of the organic wastewater compounds that were detected in groundwater samples, caffeine and tetrachloroethene might be more easily introduced from sources other than onsite wastewater, but were among the least frequently detected (table 9). (People sometimes dispose of leftover caffeine-containing drinks and, to the detriment of water resources, dispose of solvents such as tetrachloroethene by pouring onto the ground.) Another difficulty in quantifying organic wastewater compound transport from these results is that the concentrations of organic wastewater compounds observed in the ground water are small relative to LRLs, and estimated results have lower precision than would be expected at concentrations well above LRLs. The spotty occurrence of organic wastewater compounds might also be explained by variable processes governing loading of some of these compounds to the aquifer, or variable initial source concentrations. Specifically, although nitrogen and chloride concentrations in onsite wastewater are consistently similar among systems, organic wastewater compound concentrations in onsite wastewater are highly variable among the different systems (table 6). Source composition variability might partly result from nonuniform use of products containing organic wastewater compounds. This variability in organic wastewater compound concentrations among systems might be representative of temporal variability in organic wastewater compound concentrations for individual systems. The common approach to characterizing nitrate transport from onsite wastewater treatment systems is to assume that nitrogen loading occurs at a relatively constant rate. This assumption may not be feasible for many organic wastewater compounds. In other words, the spatially variable occurrence of organic wastewater compounds along the transects indicates that organic wastewater compound loading to aquifers might be temporally highly variable. Such highly variable source terms might not be as amenable to transport modeling as would be more uniformly loaded contaminants such as nitrogen. In this study, though, temporal variability of organic wastewater compounds in individual onsite wastewater treatment systems was not measured, and it is not clear at this time that organic wastewater compound concentrations in individual onsite wastewater treatment systems would exhibit the same degree 


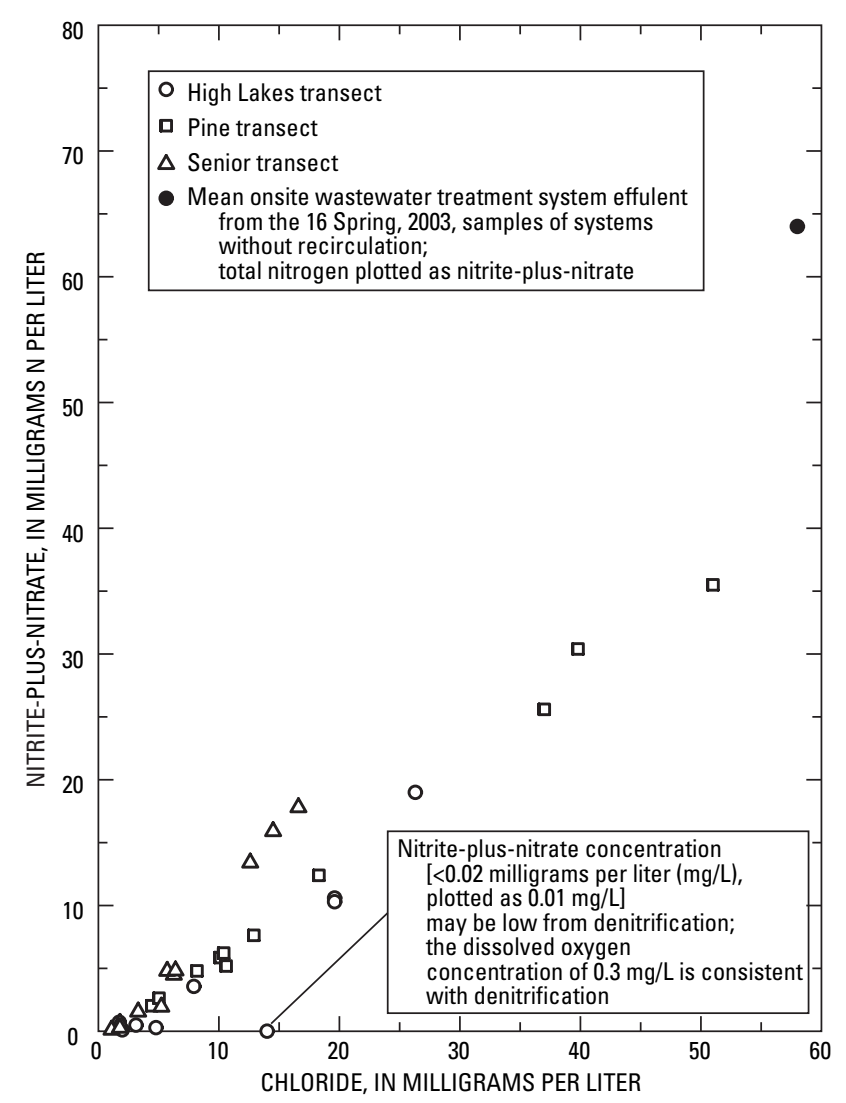

Figure 5. Relation between chloride and nitrite-plus-nitrate concentrations for the three transects near La Pine, Oregon, 2003.

of variability as was observed among different onsite wastewater treatment systems.

\section{Pharmaceuticals}

Ground-water samples from the Senior transect (11 monitoring wells installed along a transect, plus 1 sample from the onsite wastewater treatment system) were analyzed for a suite of 18 pharmaceuticals (14 human prescription and nonprescription medical drugs and 1 medical drug metabolite; plus caffeine, a caffeine metabolite, and a nicotine metabolite). The medical drugs in this analytical suite represent but a small fraction of the thousands of medical drugs in routine use. Concentrations of pharmaceuticals from the Senior transect are given in table 10. Quality-control data associated with these environmental data are presented in Appendix C.

\section{Pharmaceuticals in Onsite Wastewater}

Only one onsite wastewater treatment system - the one associated with the Senior transect-was sampled for pharmaceuticals (table 10). Of the 18 pharmaceuticals analyzed for in the onsite wastewater, 8 were detected at concentrations above provisional LRLs. Concentrations ranged from less than $1 \mu \mathrm{g} / \mathrm{L}$ to an estimated $120 \mu \mathrm{g} / \mathrm{L}$ (acetaminophen). Acetaminophen had a higher estimated concentration than even caffeine (estimated $110 \mu \mathrm{g} / \mathrm{L}$ ). Use of the onsite wastewater treatment system by numerous members of the La Pine Senior Center, an unknown number of whom might be using a variety of pharmaceuticals, increases the potential number of pharmaceuticals that might be loaded into the onsite wastewater treatment system. Thus, this single analysis from the Senior Center wastewater treatment system may not be representative of the loading from the typical single-family onsite wastewater treatment system.

\section{Pharmaceuticals in Transect Samples}

Monitoring wells installed along the La Pine Senior Center plume of onsite wastewater were sampled and the water analyzed for pharmaceuticals to provide information on pharmaceutical occurrence and transport in ground water (table 10). Although transect monitoring well samples generally contained elevated concentrations of chloride and nitrate, pharmaceuticals were infrequently detected - three pharmaceuticals were detected at concentrations above provisional LRLs, each only one time (the Senior 10 sample containing acetaminophen at $0.12 \mu \mathrm{g} / \mathrm{L}$ and caffeine at $0.18 \mu \mathrm{g} / \mathrm{L}$, and the Senior 2 sample containing sulfamethoxazole at $0.10 \mu \mathrm{g} / \mathrm{L}$ ). These concentrations are in the range of concentrations previously reported for wastewater-impacted ground water (typically sub-microgram-per-liter concentrations; Zwiener and others, 2001). The detections were in samples that contained only modest concentrations of chloride (on the order of 3 to $6 \mathrm{mg} / \mathrm{L}$ ) and nitrate (on the order of 2 to $4 \mathrm{mg} \mathrm{N} / \mathrm{L}$ ). Samples with higher concentrations of chloride (up to $16.6 \mathrm{mg} / \mathrm{L}$ ) and nitrate (up to $17.8 \mathrm{mg} \mathrm{N} / \mathrm{L}$ ) did not contain pharmaceuticals at concentrations above provisional LRLs. The spatially variable occurrence of pharmaceuticals along the transects suggests that pharmaceutical loading to aquifers may be variable in time (i.e., associated with episodic discharge), in a similar manner as was postulated earlier in this report for organic wastewater compounds.

In addition to pharmaceutical results from the Senior transect, two pharmaceuticals - the anticonvulsant drugs primidone and phenobarbitol—were tentatively identified in three of the High Lakes transect ground-water samples (table 11). The tentative identification of primidone and phenobarbitol occurred during the process of analyzing ground-water samples for organic wastewater compounds. In analyzing water samples for organic wastewater compounds by GC/MS, gas chromatography retention times and mass spectrometry spectra are compared to the gas chromatography retention times and mass spectrometry spectra of target organic wastewater compound calibration standards analyzed on the same GC/MS apparatus being used to analyze the environmental samples. It is the specific matches of gas chromatography 
Table 10. Concentrations of pharmaceuticals in ground water along Senior transect, La Pine, Oregon, 2003.

[STE, Septic tank effluent; Date as year, month, day (YYYYMMDD); Time in hours and minutes, military; Volume in milliliters; Chloride in milligrams per liter; Nitrite-plus-nitrate in milligrams N per liter; Pharmaceuticals in micrograms per liter; "<", less than; "E", estimated (for cimetidine, estimated because recovery in laboratory spiked reagent grade water averages <60 percent; for other analytes, estimated because concentrations were greater than the highest calibration standard); archived extract of Senior 10 sample was reanalyzed December 5, 2003, confirming presence of acetaminophen ( 0.08 micrograms per liter) and caffeine ( 0.11 micrograms per liter); sulfamethoxazole was reported in the Senior 2 sample but not in the Senior STE sample; the sulfamethoxazole parent ion was present in the Senior STE sample at a concentration of 0.28 micrograms per liter but the confirmation ion was buried, and thus sulfamethoxazole was reported as a nondetect; - , no data]

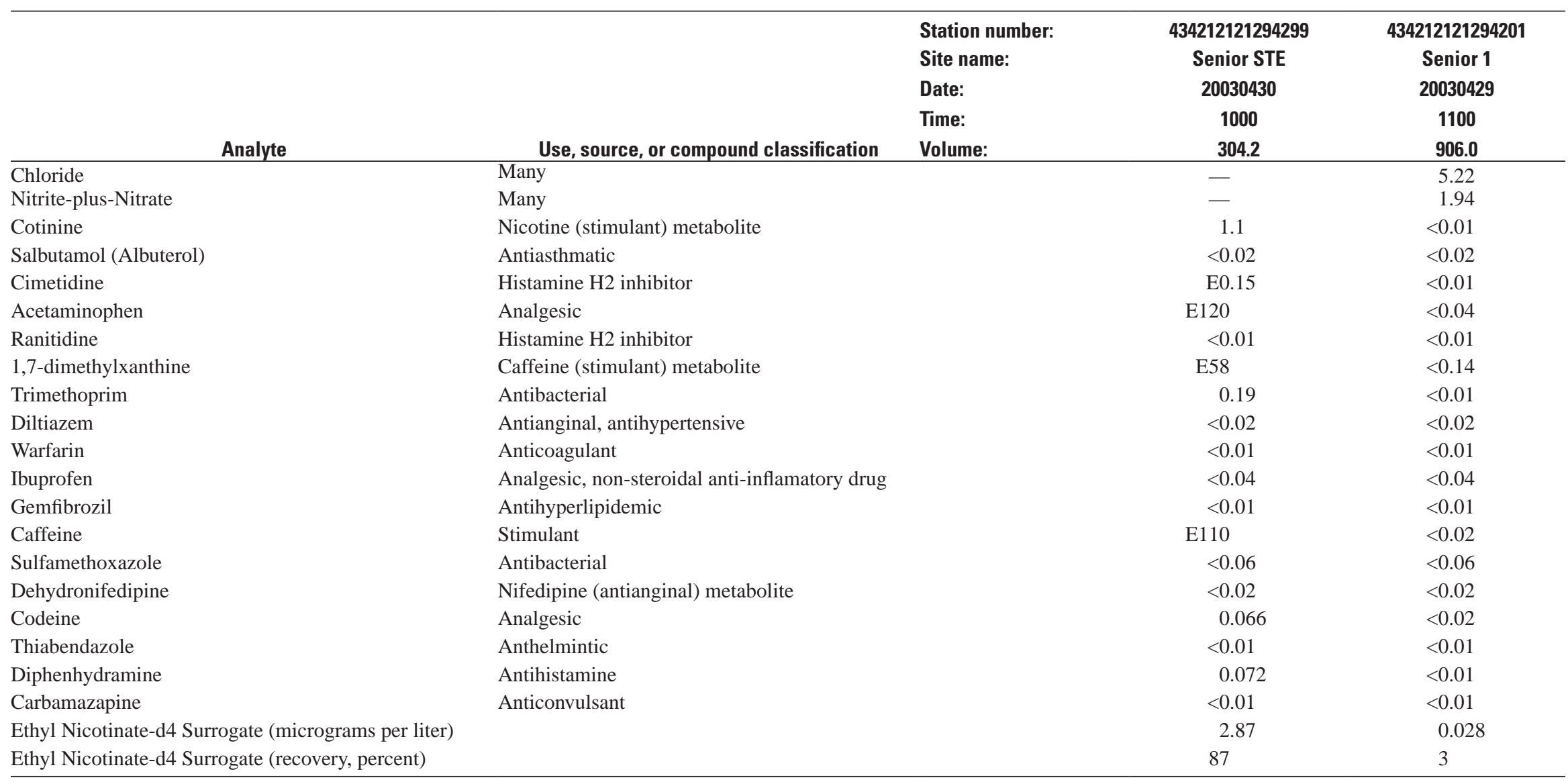


Table 10. Concentrations of pharmaceuticals in ground water along Senior transect, La Pine, Oregon, 2003.-Continued

[STE, Septic tank effluent; Date as year, month, day (YYYYMMDD); Time in hours and minutes, military; Volume in milliliters; Chloride in milligrams per liter; Nitrite-plus-nitrate in milligrams N per liter; Pharmaceuticals in micrograms per liter; "<", less than; "E", estimated (for cimetidine, estimated because recovery in laboratory spiked reagent grade water averages <60 percent; for other analytes, estimated because concentrations were greater than the highest calibration standard); archived extract of Senior 10 sample was reanalyzed December 5, 2003, confirming presence of acetaminophen ( 0.08 micrograms per liter) and caffeine (0.11 micrograms per liter); sulfamethoxazole was reported in the Senior 2 sample but not in the Senior STE sample; the sulfamethoxazole parent ion was present in the Senior STE sample at a concentration of 0.28 micrograms per liter but the confirmation ion was buried, and thus sulfamethoxazole was reported as a nondetect; - , no data]

\begin{tabular}{|c|c|c|c|c|c|}
\hline & $\begin{array}{c}434212121294202 \\
\text { Senior } 2\end{array}$ & $\begin{array}{c}434212121294203 \\
\text { Senior } 3\end{array}$ & $\begin{array}{c}434212121294204 \\
\text { Senior } 4\end{array}$ & $\begin{array}{c}434212121294205 \\
\text { Senior } 5\end{array}$ & $\begin{array}{c}434212121294206 \\
\text { Senior } 6\end{array}$ \\
\hline & 20030429 & 20030430 & 20030430 & 20030430 & 20030501 \\
\hline & 1300 & 1300 & 1600 & 1800 & 1000 \\
\hline Analyte & 954.6 & 954.8 & 927.3 & 970.7 & 958.7 \\
\hline Chloride & 6.26 & 14.5 & 5.71 & 16.6 & 6.40 \\
\hline Nitrite-plus-Nitrate & 4.48 & 15.9 & 4.79 & 17.8 & 4.82 \\
\hline Cotinine & $<0.01$ & $<0.01$ & $<0.01$ & $<0.01$ & $<0.01$ \\
\hline Salbutamol (Albuterol) & $<0.02$ & $<0.02$ & $<0.02$ & $<0.02$ & $<0.02$ \\
\hline Cimetidine & $<0.01$ & $<0.01$ & $<0.01$ & $<0.01$ & $<0.01$ \\
\hline Acetaminophen & $<0.04$ & $<0.04$ & $<0.04$ & $<0.04$ & $<0.04$ \\
\hline Ranitidine & $<0.01$ & $<0.01$ & $<0.01$ & $<0.01$ & $<0.01$ \\
\hline 1,7-dimethylxanthine & $<0.14$ & $<0.14$ & $<0.14$ & $<0.14$ & $<0.14$ \\
\hline Trimethoprim & $<0.01$ & $<0.01$ & $<0.01$ & $<0.01$ & $<0.01$ \\
\hline Diltiazem & $<0.02$ & $<0.02$ & $<0.02$ & $<0.02$ & $<0.02$ \\
\hline Warfarin & $<0.01$ & $<0.01$ & $<0.01$ & $<0.01$ & $<0.01$ \\
\hline Ibuprofen & $<0.04$ & $<0.04$ & $<0.04$ & $<0.04$ & $<0.04$ \\
\hline Gemfibrozil & $<0.01$ & $<0.01$ & $<0.01$ & $<0.01$ & $<0.01$ \\
\hline Caffeine & $<0.02$ & $<0.02$ & $<0.02$ & $<0.02$ & $<0.02$ \\
\hline Sulfamethoxazole & 0.10 & $<0.06$ & $<0.06$ & $<0.06$ & $<0.06$ \\
\hline Dehydronifedipine & $<0.02$ & $<0.02$ & $<0.02$ & $<0.02$ & $<0.02$ \\
\hline Codeine & $<0.02$ & $<0.02$ & $<0.02$ & $<0.02$ & $<0.02$ \\
\hline Thiabendazole & $<0.01$ & $<0.01$ & $<0.01$ & $<0.01$ & $<0.01$ \\
\hline Diphenhydramine & $<0.01$ & $<0.01$ & $<0.01$ & $<0.01$ & $<0.01$ \\
\hline Carbamazapine & $<0.01$ & $<0.01$ & $<0.01$ & $<0.01$ & $<0.01$ \\
\hline Ethyl Nicotinate-d4 Surrogate (micrograms per liter) & 0.78 & 0.54 & 0.86 & 0.73 & 0.76 \\
\hline Ethyl Nicotinate-d4 Surrogate (recovery, percent) & 75 & 52 & 80 & 71 & 73 \\
\hline
\end{tabular}


Table 10. Concentrations of pharmaceuticals in ground water along Senior transect, La Pine, Oregon, 2003.—Continued

[STE, Septic tank effluent; Date as year, month, day (YYYYMMDD); Time in hours and minutes, military; Volume in milliliters; Chloride in milligrams per liter; Nitrite-plus-nitrate in milligrams N per liter; Pharmaceuticals in micrograms per liter; "<", less than; "E", estimated (for cimetidine, estimated because recovery in laboratory spiked reagent grade water averages <60 percent; for other analytes, estimated because concentrations were greater than the highest calibration standard); archived extract of Senior 10 sample was reanalyzed December 5 , 2003, confirming presence of acetaminophen ( 0.08 micrograms per liter) and caffeine ( 0.11 micrograms per liter); sulfamethoxazole was reported in the Senior 2 sample but not in the Senior STE sample; the sulfamethoxazole parent ion was present in the Senior STE sample at a concentration of 0.28 micrograms per liter but the confirmation ion was buried, and thus sulfamethoxazole was reported as a nondetect; - , no data]

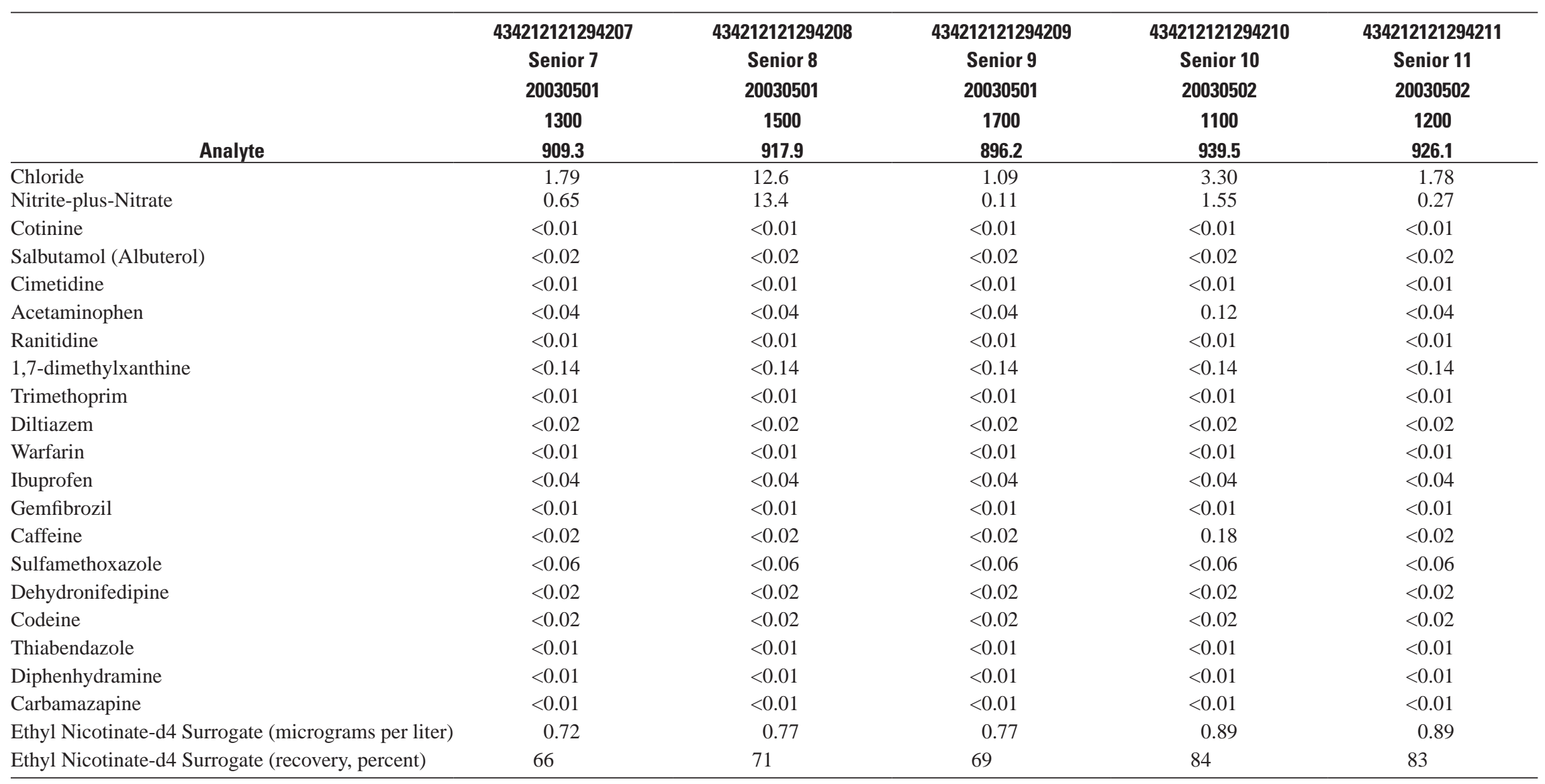


retention times and mass spectrometry spectra of environmental samples to those of calibration standards that allows identification and quantification of the target compounds. During GC/MS, if chromatogram peaks not associated with the target compounds are observed, the mass spectra of the unidentified compounds can sometimes be matched to known mass spectra in a mass spectral reference library and thus be tentatively identified and quantified. Such identification is considered tentative because calibration standards for such identified compounds were not analyzed on the same GC/MS apparatus used to analyze the environmental samples. The reported concentrations are considered semiquantitative, but the reported concentrations are generally accurate to within one order of magnitude.

The tentative identification of two pharmaceuticals in each of three wells at one well nest, and an absence of identification in any other ground-water samples, again indicates the heterogeneous nature of wastewater-derived compound occurrence in La Pine ground water. The co-occurrence of two drugs used as anticonvulsants could reflect upgradient use of both drugs. However, in addition to being an anticonvulsant, phenobarbitol also is a metabolite of primidone (Sidki and others, 1985), and it may have been present due to the presence of primidone. Phenobarbitol concentrations were estimated to be 8 to 20 percent of primidone concentrations (table 11).

In a study of ground-water transport of a suite of pharmaceuticals that included two anticonvulsant drugs (primidone and carbamazepine), Drewes and others (2003) found that these anticonvulsant drugs were transported nearly conservatively and to a greater extent than the other pharmaceuticals evaluated. Others also have detected anticonvulsant drugs in onsite-wastewater-impacted ground water (Seiler and others, 1999) and in lake water that receives municipal wastewater (the same two anticonvulsant drugs tentatively detected in the La Pine samples: primidone and phenobarbitol; Snyder and others, 2001). The tentative identification of the anticonvulsant drugs primidone and phenobarbitol in onsite-waste- water-impacted ground water in La Pine adds to this body of evidence suggesting that some anticonvulsant drugs might be useful indicators of human wastewater dispersed in the aquatic environment. Furthermore, the apparent persistence of some anticonvulsant drugs in the aquatic environment may indicate a need for scientists and resource managers to consider the potential for adverse health effects of anticonvulsant drugs on aquatic organisms and on human consumers of water impacted by onsite wastewater.

In an occurrence survey of 17 monitoring, domestic, and municipal wells for a suite of pharmaceuticals, Seiler and others (1999) concluded that pharmaceuticals were useful indicators of onsite wastewater, but would be of limited use as tracers of onsite wastewater because of the unpredictable nature of pharmaceutical occurrence. Organic wastewater compound and pharmaceutical occurrence along the La Pine transects provide insight into the transport behavior of these compounds along well-defined plumes of onsite wastewater. In addition to degradation and sorption, these results indicate that organic wastewater compound and pharmaceutical occurrence might reflect temporally variable loading rates. Results from the La Pine ground-water flowpath-based sampling program are consistent with the conclusions of Seiler and others (1999).

\section{Coliphage}

Concentrations of coliphage from the innovative system network (28 onsite wastewater treatment systems, 5 downgradient lysimeters, and 28 drainfield monitoring wells) are listed in table 12. Coliphage concentrations from the transects (31 monitoring wells installed along three transects, along with a sample from 1 of the 3 onsite wastewater treatment systems for these transects) are given in table 13. Quality-control data associated with these environmental data are presented in Appendix D.

Table 11. List of pharmaceuticals tentatively identified during analysis of ground-water samples for organic wastewater compounds, La Pine, Oregon, 2003.

[Date as year, month, day (YYYYMMDD); Time in hours and minutes, military; CAS, chemical abstract number; data for tentatively identified compounds in this report are based on comparison of sample spectra with library spectra followed by visual examination by GC/MS analysts. Tentatively identified compound data have not been confirmed by direct comparison with reference standards. Therefore, identification is tentative, and reported concentrations are semiquantitative, although reported concentrations generally are accurate to one order of magnitude; $\mu \mathrm{g} / \mathrm{L}$, micrograms per liter]

\begin{tabular}{ccccc}
\hline $\begin{array}{c}\text { Transect } \\
\text { well name }\end{array}$ & Station number & Date and time & $\begin{array}{c}\text { Primidone } \\
(\mathbf{C A S} \text { 125-33-7) } \\
(\mu \mathbf{g} / \mathbf{L})\end{array}$ & $\begin{array}{c}\text { Phenobarbitol } \\
\text { (CAS 50-06-6) } \\
(\mu \mathbf{g} / \mathbf{L})\end{array}$ \\
\hline High Lakes 3 & 434241121311603 & 200306111200 & 12 & 1.0 \\
High Lakes 4 & 434241121311604 & 200306111500 & 2.0 & 0.4 \\
High Lakes 5 & 434241121311605 & 200306111600 & 0.5 & 0.1 \\
\hline
\end{tabular}


Table 12. Coliphage data for water from traditional and innovative onsite wastewater treatment systems, downgradient lysimeters, and downgradient ground water, La Pine, Oregon, 2003.

[Sample type: STE, septic tank effluent, EOP, end of pipe (end of innovative treatment), SFE, sand filter effluent, LYS, lysimeter, DFMW, drainfield monitoring well; date as year, month, day (YYYYMMDD); time in hours and minutes, military; mg/L, milligrams per liter; mg N/L, milligrams nitrogen per liter; $\mathrm{mL}$, milliliter; total nitrogen data are provided for STE and EOP samples, whereas nitrite-plus-nitrate data are provided for SFE, LYS and DFMW samples because these oxidized nitrogen species are the dominant nitrogen species in these samples; "E", estimated; <, less than; >, greater than; --, no data]

\begin{tabular}{|c|c|c|c|c|c|c|c|c|c|c|c|c|c|c|}
\hline \multirow[b]{2}{*}{$\begin{array}{c}\text { Type of onsite } \\
\text { system }^{\mathrm{a}}\end{array}$} & \multirow[b]{2}{*}{$\begin{array}{c}\text { Sample } \\
\text { type }\end{array}$} & \multirow[b]{2}{*}{ Station number } & \multirow[b]{2}{*}{$\begin{array}{c}\text { Sample } \\
\text { date/time }\end{array}$} & \multirow[b]{2}{*}{$\begin{array}{l}\text { Chloride } \\
(\mathrm{mg} / \mathrm{L})^{b}\end{array}$} & \multirow[b]{2}{*}{$\begin{array}{c}\text { Nitrite-plus- } \\
\text { nitrate } \\
(\mathrm{mg} \mathrm{N} / \mathrm{L})^{\mathrm{c}}\end{array}$} & \multirow[b]{2}{*}{$\begin{array}{c}\text { Total nitrogen } \\
(\mathrm{mg} \mathrm{N} / \mathrm{L})^{d}\end{array}$} & \multicolumn{2}{|c|}{ Method 1602-Single-agar layer } & \multirow[b]{2}{*}{$\begin{array}{c}\text { Sample } \\
\text { date/time }\end{array}$} & \multirow[b]{2}{*}{$\begin{array}{c}\text { Chloride }^{b} \\
\text { (mg/L) }\end{array}$} & \multirow[b]{2}{*}{$\begin{array}{c}\text { Nitrite-plus- } \\
\text { nitrate } \\
(\mathrm{mg} \mathrm{N} / \mathrm{L})^{\mathrm{c}}\end{array}$} & \multirow[b]{2}{*}{$\begin{array}{c}\text { Total } \\
\text { nitrogen } \\
(\mathrm{mg} \mathrm{N} / \mathrm{L})^{\mathrm{d}}\end{array}$} & \multicolumn{2}{|c|}{ Method 1602-Single-agar layer } \\
\hline & & & & & & & $\begin{array}{c}\text { F-Specific } \\
\text { Coliphage } \\
\text { (USGS paramete } \\
\text { code 90904; } \\
\text { plaques per } \\
100 \mathrm{~mL} \text { ) } \\
\end{array}$ & $\begin{array}{c}\text { Somatic } \\
\text { Coliphage } \\
\text { (USGS parameter } \\
\text { code 90903; } \\
\text { plaques per } \\
100 \mathrm{~mL} \text { ) } \\
\end{array}$ & & & & & $\begin{array}{c}\text { F-Specific } \\
\text { Coliphage } \\
\text { (USGS parameter } \\
\text { code 90904; } \\
\text { plaques per } \\
100 \mathrm{~mL} \text { ) } \\
\end{array}$ & $\begin{array}{c}\text { Somatic } \\
\text { Coliphage } \\
\text { (USGS parameter } \\
\text { code } 90903 ; \\
\text { plaques per } \\
100 \mathrm{~mL} \text { ) } \\
\end{array}$ \\
\hline \multirow[t]{3}{*}{ Standard } & STE & 434207121324602 & 200304140900 & 96 & - & 99 & $<1$ & $20,000^{\mathrm{c}}$ & 200312080900 & 193 & - & - & $<1$ & 300 \\
\hline & LYS & 434207121324605 & 200304140900 & 85 & 67.2 & - & $<1$ & 360 & 200312080900 & 205 & - & - & $<1$ & 140 \\
\hline & DFMW & 434207121324601 & 200304140900 & 16 & 7.3 & - & $<1$ & $<1$ & 200312080900 & 27.5 & - & - & $<1$ & $<1$ \\
\hline \multirow[t]{3}{*}{ Standard } & STE & 434236121310502 & 200306040800 & 27 & - & 71 & $<1$ & $<1$ & 200312080930 & 25.8 & - & - & $<1$ & $<1$ \\
\hline & LYS & 434236121310505 & 200306040800 & 25 & 32.7 & - & $<1$ & 1 & 200312080930 & 25.3 & - & - & $<1$ & $<1$ \\
\hline & DFMW & 434236121310501 & 200306040800 & 5.2 & 4.0 & - & $<1$ & $<1$ & 200312080930 & 5.33 & - & - & $<1$ & $<1$ \\
\hline \multirow[t]{3}{*}{ Pressure } & STE & 434247121305502 & 200305141100 & 25 & - & 41 & $<1$ & 4 & 200312081000 & 34.2 & - & - & $<1$ & 200 \\
\hline & LYS & 434247121305505 & 200305141100 & 28 & 39.0 & - & $<1$ & $<1$ & 200312081000 & 31.1 & - & - & 1 & $<1$ \\
\hline & DFMW & 434247121305501 & 200305141100 & 29 & 0.09 & - & $<1$ & $<1$ & 200312081000 & 30.2 & - & - & $<1$ & $<1$ \\
\hline \multirow[t]{3}{*}{ Pressure } & STE & 434248121295902 & 200304070900 & 35 & - & 58 & $1,500^{\mathrm{e}}$ & 3 & 200312081030 & 28.2 & - & - & $270,000^{\mathrm{e}}$ & 2 \\
\hline & LYS & 434248121295905 & 200304070900 & 34 & 43.7 & - & $<1$ & $<1$ & 200312081030 & 29.2 & - & - & $<1$ & $<1$ \\
\hline & DFMW & 434248121295901 & 200304070900 & 4.0 & 0.37 & - & $<1$ & $<1$ & 200312081030 & 5.98 & - & - & $<1$ & $<1$ \\
\hline \multirow[t]{3}{*}{ Sand Filter } & STE & 434347121293902 & 200304141200 & 100 & - & 76 & $<1$ & $270^{\mathrm{e}}$ & 200310130900 & 60 & - & 100 & $<1$ & 350 \\
\hline & SFE & 434347121293903 & 200304141200 & 98 & 71.5 & - & $<1$ & $<1$ & 200310130900 & 60 & 150 & - & $<1$ & $<1$ \\
\hline & DFMW & 434347121293901 & 200304141200 & 48 & 71.7 & - & $<1$ & $<1$ & 200310130900 & 60 & 61.7 & - & $<1$ & $<1$ \\
\hline \multirow[t]{3}{*}{ Sand Filter } & STE & 434741121273401 & 200304071100 & 37 & - & 56 & $<1$ & $<1$ & - & - & - & - & - & - \\
\hline & SFE & 434741121273501 & 200304071100 & 32 & 58.1 & - & $<1$ & $<1$ & - & - & - & - & - & - \\
\hline & DFMW & 434741121273101 & 200304071100 & 8.6 & 7.2 & - & $<1$ & $<1$ & - & - & - & - & - & - \\
\hline \multirow[t]{3}{*}{ AdvanTex (AX-20) } & STE & 434652121273002 & 200304141100 & 46 & - & 20 & $<1$ & $<1$ & 200310061000 & 39 & - & 14 & $<1$ & $<1$ \\
\hline & EOP & 434652121273004 & 200304141100 & 44 & - & 21 & $<1$ & $<1$ & 200310061000 & 39 & - & 16 & $<1$ & $<1$ \\
\hline & DFMW & 434652121273001 & 200304141100 & 5.9 & 3.1 & - & $<1$ & $<1$ & 200310061000 & 3.9 & 2.2 & - & $<1$ & $<1$ \\
\hline
\end{tabular}


Table 12. Coliphage data for water from traditional and innovative onsite wastewater treatment systems, downgradient lysimeters, and downgradient ground water, La Pine, Oregon, 2003.-Continued

[Sample type: STE, septic tank effluent, EOP, end of pipe (end of innovative treatment), SFE, sand filter effluent, LYS, lysimeter, DFMW, drainfield monitoring well; date as year, month, day (YYYYMMDD); time in hours and minutes, military; $\mathrm{mg} / \mathrm{L}$, milligrams per liter; $\mathrm{mg} \mathrm{N} / \mathrm{L}$, milligrams nitrogen per liter; $\mathrm{mL}$, milliliter; total nitrogen data are provided for STE and EOP samples, whereas nitrite-plus-nitrate data are provided for SFE, LYS and DFMW samples because these oxidized nitrogen species are the dominant nitrogen species in these samples; "E", estimated; <, less than; >, greater than; --, no data]

\begin{tabular}{|c|c|c|c|c|c|c|c|c|c|c|c|c|c|c|}
\hline \multirow[b]{2}{*}{$\begin{array}{c}\text { Type of onsite } \\
\text { system }^{\mathrm{a}}\end{array}$} & \multirow[b]{2}{*}{$\begin{array}{c}\text { Sample } \\
\text { type }\end{array}$} & \multirow[b]{2}{*}{ Station number } & \multirow[b]{2}{*}{$\begin{array}{c}\text { Sample } \\
\text { date/time }\end{array}$} & \multirow[b]{2}{*}{$\begin{array}{l}\text { Chloride } \\
(\mathrm{mg} / \mathrm{L})^{\mathrm{b}}\end{array}$} & \multirow[b]{2}{*}{$\begin{array}{c}\text { Nitrite-plus- } \\
\text { nitrate } \\
(\mathrm{mg} \mathrm{N} / \mathrm{L})^{\mathrm{c}}\end{array}$} & \multirow[b]{2}{*}{$\begin{array}{l}\text { Total nitrogen } \\
(\mathbf{m g ~ N} / \mathbf{L})^{d}\end{array}$} & \multicolumn{2}{|c|}{ Method 1602-Single-agar layer } & \multirow[b]{2}{*}{$\begin{array}{c}\text { Sample } \\
\text { date/time }\end{array}$} & \multirow[b]{2}{*}{$\begin{array}{c}\text { Chloride }^{\mathrm{b}} \\
\text { (mg/L) }\end{array}$} & \multirow[b]{2}{*}{$\begin{array}{c}\text { Nitrite-plus- } \\
\text { nitrate } \\
(\mathrm{mg} \mathrm{N} / \mathrm{L})^{\mathrm{c}}\end{array}$} & \multirow[b]{2}{*}{$\begin{array}{c}\text { Total } \\
\text { nitrogen } \\
(\mathrm{mg} \mathrm{N} / \mathrm{L})^{\mathrm{d}}\end{array}$} & \multicolumn{2}{|c|}{ Method 1602-Single-agar layer } \\
\hline & & & & & & & $\begin{array}{c}\text { F-Specific } \\
\text { Coliphage } \\
\text { (USGS paramete } \\
\text { code } 90904 ; \\
\text { plaques per } \\
100 \mathrm{~mL} \text { ) }\end{array}$ & $\begin{array}{l}\text { Somatic } \\
\text { Coliphage } \\
\text { r (USGS parameter } \\
\text { code 90903; } \\
\text { plaques per } \\
100 \mathrm{~mL} \text { ) }\end{array}$ & & & & & $\begin{array}{c}\text { F-Specific } \\
\text { Coliphage } \\
\text { (USGS parameter } \\
\text { code 90904; } \\
\text { plaques per } \\
100 \mathrm{~mL} \text { ) }\end{array}$ & $\begin{array}{c}\text { Somatic } \\
\text { Coliphage } \\
\text { (USGS parameter } \\
\text { code 90903; } \\
\text { plaques per } \\
100 \mathrm{~mL} \text { ) }\end{array}$ \\
\hline \multirow[t]{3}{*}{ AdvanTex (AX-20) } & ) STE & 434536121291202 & 200306040900 & 39 & - & 20 & $<1$ & 10 & 200311051100 & - & - & - & $<1$ & 13 \\
\hline & EOP & 434536121291204 & 200306040900 & 38 & - & 9.1 & $<1$ & 5 & 200311051100 & - & - & - & $<1$ & 10 \\
\hline & DFMW & 434536121291201 & 200306040900 & 0.6 & 0.002 & - & $<1$ & $<1$ & 200311051100 & - & - & - & $<1$ & $<1$ \\
\hline \multirow[t]{3}{*}{ Amphidrome } & EOP & 434011121314604 & 200304230900 & 90 & - & 31 & 5 & 2 & 200311170900 & - & - & - & 640 & $<1$ \\
\hline & DFMW & 434011121314601 & 200304230900 & 58 & 7.6 & - & $<1$ & 1 & 200311170900 & - & - & - & $<1$ & $<1$ \\
\hline & $\begin{array}{l}\text { DFMW } \\
\text { resample }\end{array}$ & 434011121314601 & 200305270900 & - & - & - & - & $<1^{\mathrm{f}}$ & - & - & - & - & - & - \\
\hline \multirow[t]{3}{*}{ Amphidrome } & EOP & 434243121290104 & 200304231000 & - & - & 44 & $\mathrm{E} 7,500^{\mathrm{g}}$ & 1 & 200311051100 & - & - & - & $3,300^{\mathrm{e}}$ & 59 \\
\hline & DFMW & 434243121290101 & 200304231000 & 19 & 7.1 & - & $<1$ & 8 & 200311051100 & - & - & - & $<1$ & $<1$ \\
\hline & $\begin{array}{l}\text { DFMW } \\
\text { resample }\end{array}$ & 434243121290101 & 200305271000 & - & - & - & - & $<1^{\mathrm{f}}$ & - & - & - & - & - & - \\
\hline \multirow[t]{3}{*}{ Biokreisel } & STE & 434226121293302 & 200304141000 & 46 & - & 20 & 19 & $9,900^{\mathrm{e}}$ & 200310131000 & - & - & - & 200 & 1,400 \\
\hline & EOP & 434226121293304 & 200304141000 & 44 & - & 15 & 16 & 580 & 200310131000 & 61 & - & 4.8 & 83 & 580 \\
\hline & DFMW & 434226121293301 & 200304141000 & 13 & 1.8 & - & $<1$ & $<1$ & 200310131000 & 11 & 0.8 & - & $<1$ & $<1$ \\
\hline \multirow[t]{3}{*}{ Biokreisel } & STE & 434727121273702 & 200306041100 & 35 & - & 26 & $<1$ & 60 & 200312011100 & 44.2 & - & - & $<1$ & 640 \\
\hline & EOP & 434727121273704 & 200306041100 & 32 & - & 7.4 & $<1$ & $<1$ & 200312011100 & 39.6 & - & - & $<1$ & 7 \\
\hline & DFMW & 434727121273701 & 200306041100 & 30 & 11.7 & - & $<1$ & $<1$ & 200312011100 & 41.1 & - & - & $<1$ & $<1$ \\
\hline \multirow[t]{4}{*}{ Enviroserver } & STE & 434836121271102 & 200304231100 & 47 & - & 178 & $<1$ & $<1$ & 200310131200 & 28 & - & 272 & $<1$ & 100 \\
\hline & EOP & 434836121271104 & 200304231100 & 59 & - & 16 & $<1$ & $<1$ & 200310131200 & 32 & - & 8.4 & $<1$ & 5 \\
\hline & DFMW & 434836121271101 & 200304231100 & 61 & 15.9 & - & $<1$ & 1 & 200310131200 & 150 & 0.8 & - & $<1$ & $<1$ \\
\hline & $\begin{array}{l}\text { DFMW } \\
\text { resample }\end{array}$ & 434836121271101 & 200305271200 & - & - & - & - & $<1^{\mathrm{f}}$ & - & - & - & - & - & - \\
\hline \multirow[t]{3}{*}{ Enviroserver } & STE & 433855121300102 & 200304230900 & 44 & - & 37 & $<1$ & 220 & 200310131100 & 38 & - & 113 & $<1$ & 19 \\
\hline & EOP & 433855121300104 & 200304230900 & 45 & - & 34 & $<1$ & 420 & 200310131100 & 36 & - & 23 & $<1$ & 39 \\
\hline & DFMW & 433855121300101 & 200304230900 & 3.9 & 1.2 & - & $<1$ & $<1$ & 200310131100 & 4.8 & 1.6 & - & $<1$ & $<1$ \\
\hline
\end{tabular}


Table 12. Coliphage data for water from traditional and innovative onsite wastewater treatment systems, downgradient lysimeters, and downgradient ground water, La Pine, Oregon, 2003.-Continued

[Sample type: STE, septic tank effluent, EOP, end of pipe (end of innovative treatment), SFE, sand filter effluent, LYS, lysimeter, DFMW, drainfield monitoring well; date as year, month, day (YYYYMMDD); time in hours and minutes, military; $\mathrm{mg} / \mathrm{L}$, milligrams per liter; $\mathrm{mg} \mathrm{N} / \mathrm{L}$, milligrams nitrogen per liter; $\mathrm{mL}$, milliliter; total nitrogen data are provided for STE and EOP samples, whereas nitrite-plus-nitrate data are provided for SFE, LYS and DFMW samples because these oxidized nitrogen species are the dominant nitrogen species in these samples; "E", estimated; <, less than; >, greater than; --, no data]

\begin{tabular}{|c|c|c|c|c|c|c|c|c|c|c|c|c|c|c|}
\hline \multirow[b]{2}{*}{$\begin{array}{c}\text { Type of onsite } \\
\text { system }^{\mathrm{a}}\end{array}$} & \multirow[b]{2}{*}{$\begin{array}{c}\text { Sample } \\
\text { type }\end{array}$} & \multirow[b]{2}{*}{ Station number } & \multirow[b]{2}{*}{$\begin{array}{c}\text { Sample } \\
\text { date/time }\end{array}$} & \multirow[b]{2}{*}{$\begin{array}{l}\text { Chloride } \\
(\mathrm{mg} / \mathrm{L})^{b}\end{array}$} & \multirow[b]{2}{*}{$\begin{array}{c}\text { Nitrite-plus- } \\
\text { nitrate } \\
(\mathrm{mg} \mathrm{N} / \mathrm{L})^{c}\end{array}$} & \multirow[b]{2}{*}{$\begin{array}{c}\text { Total nitrogen } \\
(\mathrm{mg} \mathrm{N} / \mathrm{L})^{d}\end{array}$} & \multicolumn{2}{|c|}{ Method 1602-Single-agar layer } & \multirow[b]{2}{*}{$\begin{array}{c}\text { Sample } \\
\text { date/time }\end{array}$} & \multirow[b]{2}{*}{$\begin{array}{c}\begin{array}{c}\text { Chloride } \\
\text { (mg/L) }\end{array} \\
\text { (mg }\end{array}$} & \multirow[b]{2}{*}{$\begin{array}{c}\text { Nitrite-plus- } \\
\text { nitrate } \\
\text { (mg N/L) }\end{array}$} & \multirow[b]{2}{*}{$\begin{array}{c}\text { Total } \\
\text { nitrogen } \\
\text { (mg N/L) }\end{array}$} & \multicolumn{2}{|c|}{ Method 1602-Single-agar layer } \\
\hline & & & & & & & $\begin{array}{c}\text { F-Specific } \\
\text { Coliphage } \\
\text { (USGS parameter } \\
\text { code } 90904 ; \\
\text { plaques per } \\
100 \mathrm{~mL} \text { ) } \\
\end{array}$ & $\begin{array}{c}\text { Somatic } \\
\text { Coliphage } \\
\text { (USGS parameter } \\
\text { code 90903; } \\
\text { plaques per } \\
100 \mathrm{~mL} \text { ) } \\
\end{array}$ & & & & & $\begin{array}{c}\text { F-Specific } \\
\text { Coliphage } \\
\text { (USGS parameter } \\
\text { code 90904; } \\
\text { plaques per } \\
100 \mathrm{~mL} \text { ) } \\
\end{array}$ & \begin{tabular}{c}
\multicolumn{2}{c}{ Somatic } \\
Coliphage (USGS \\
parameter code 90903; \\
plaques per \\
$100 \mathrm{~mL}$ ) \\
\end{tabular} \\
\hline \multirow[t]{3}{*}{$\begin{array}{l}\text { FAST } \\
\end{array}$} & STE & 434952121290602 & 200304071100 & 29 & - & 64 & $3,000^{\mathrm{e}}$ & 17 & 200310061100 & 37 & - & 52 & $<1$ & $7,800^{\circ}$ \\
\hline & EOP & 434952121290604 & 200304071100 & 24 & - & 32 & 46 & $<1$ & 200310061100 & 37 & - & 32 & 840 & 130 \\
\hline & DFMW & 434952121290601 & 200304071100 & 22 & 30.5 & - & $<1$ & $<1$ & 200310061100 & 36 & 29.7 & - & $<1$ & $<1$ \\
\hline \multirow[t]{3}{*}{ FAST } & STE & 434952121290602 & - & - & - & - & - & - & 200312011200 & 33.6 & - & - & $<1$ & $2,300^{\circ}$ \\
\hline & EOP & 434952121290604 & - & - & - & - & - & - & 200312011200 & 42.4 & - & - & 220 & 79 \\
\hline & DFMW & 434952121290601 & - & - & - & - & - & - & 200312011200 & 40.5 & - & - & $<1$ & $<1$ \\
\hline \multirow[t]{3}{*}{ FAST } & STE & 434437121295302 & 200305050900 & 23 & - & 54 & $<1$ & $>1,000$ & 200311051000 & - & - & - & 33 & 540 \\
\hline & EOP & 434437121295304 & 200305050900 & 37 & - & 49 & 150 & $76,000^{\mathrm{e}}$ & 200311051000 & - & - & - & 170 & 200 \\
\hline & DFMW & 434437121295301 & 200305050900 & 17 & 0.002 & - & $<1$ & $<1$ & 200311051000 & - & - & - & $<1$ & $<1$ \\
\hline \multirow[t]{4}{*}{ NAYADIC } & STE & 435016121284702 & 200304071200 & 46 & - & 86 & $<1$ & $10,000^{\mathrm{e}}$ & 200311191200 & - & - & - & $<1$ & 5 \\
\hline & EOP & 435016121284704 & 200304071200 & 34 & - & 48 & $<1$ & $<1$ & 200311191200 & - & - & - & 1 & $<1$ \\
\hline & LYS & 435016121284705 & 200304071200 & 32 & 43.1 & - & $<1$ & 9 & 200311191200 & - & - & - & $<1$ & $<1$ \\
\hline & DFMW & 435016121284701 & 200304071200 & 9.5 & 6.3 & - & $<1$ & $<1$ & 200311191200 & - & - & - & $<1$ & $<1$ \\
\hline \multirow[t]{3}{*}{ NAYADIC } & STE & 434713121274302 & 200304231000 & 51 & - & 57 & $<1$ & 110 & 200310131000 & 160 & - & 60 & $<1$ & 14 \\
\hline & EOP & 434713121274304 & 200304231000 & 59 & - & 48 & $<1$ & 6 & 200310131000 & 170 & - & 32 & $<1$ & 28 \\
\hline & DFMW & 434713121274301 & 200304231000 & 2.9 & 0.20 & - & $<1$ & $<1$ & 200310131000 & 2.1 & 0.2 & - & $<1$ & $<1$ \\
\hline \multirow[t]{3}{*}{ Niteless } & STE & 434908121291202 & 200305191000 & 24 & - & 53 & 180 & 3 & 200311051200 & - & - & - & $<1$ & $<1$ \\
\hline & EOP & 434908121291204 & 200305191000 & 29 & - & 56 & 810 & 2 & 200311051200 & - & - & - & $<1$ & $<1$ \\
\hline & DFMW & 434908121291201 & 200305191000 & 27 & 33.1 & - & $<1$ & $<1$ & 200311051200 & - & - & - & $<1$ & $<1$ \\
\hline \multirow[t]{3}{*}{ Niteless } & STE & 434431121293502 & 200306040900 & 22 & - & 48 & $62,000^{\mathrm{e}}$ & $<1$ & 200312010900 & 37.0 & - & - & $<1$ & 7 \\
\hline & EOP & 434431121293504 & 200306040900 & 20 & - & 43 & $44,000^{\mathrm{e}}$ & $<1$ & 200312010900 & 45.0 & - & - & $<1$ & 6 \\
\hline & DFMW & 434431121293501 & 200306040900 & 31 & 34.0 & - & $<1$ & $<1$ & 200312010900 & 30.1 & - & - & $<1$ & $<1$ \\
\hline
\end{tabular}


Table 12. Coliphage data for water from traditional and innovative onsite wastewater treatment systems, downgradient lysimeters, and downgradient ground water, La Pine, Oregon, 2003. —Continued

[Sample type: STE, septic tank effluent, EOP, end of pipe (end of innovative treatment), SFE, sand filter effluent, LYS, lysimeter, DFMW, drainfield monitoring well; date as year, month, day (YYYYMMDD); time in hours and minutes, military; mg/L, milligrams per liter; $\mathrm{mg}$ N/L, milligrams nitrogen per liter; $\mathrm{mL}$, milliliter; total nitrogen data are provided for STE and EOP samples, whereas nitrite-plus-nitrate data are provided for SFE, LYS and DFMW samples because these oxidized nitrogen species are the dominant nitrogen species in these samples; "E", estimated; <, less than; >, greater than; —, no data]

\begin{tabular}{|c|c|c|c|c|c|c|c|c|c|c|c|c|c|c|}
\hline \multirow[b]{2}{*}{$\begin{array}{c}\text { Type of onsite } \\
\text { system }^{\mathrm{a}}\end{array}$} & \multirow[b]{2}{*}{$\begin{array}{c}\text { Sample } \\
\text { type }\end{array}$} & \multirow[b]{2}{*}{ Station number } & \multirow[b]{2}{*}{$\begin{array}{c}\text { Sample } \\
\text { date/time }\end{array}$} & \multirow[b]{2}{*}{$\begin{array}{l}\text { Chloride } \\
(\mathrm{mg} / \mathrm{L})^{\mathrm{b}}\end{array}$} & \multirow[b]{2}{*}{$\begin{array}{c}\text { Nitrite-plus- } \\
\text { nitrate } \\
(\mathrm{mg} \mathrm{N} / \mathrm{L})^{\mathrm{c}}\end{array}$} & \multirow[b]{2}{*}{$\begin{array}{l}\text { Total nitrogen } \\
(\mathrm{mg} \mathrm{N} / \mathrm{L})^{d}\end{array}$} & \multicolumn{2}{|c|}{ Method 1602-Single-agar layer } & \multirow[b]{2}{*}{$\begin{array}{c}\text { Sample } \\
\text { date/time }\end{array}$} & \multirow[b]{2}{*}{$\begin{array}{c}\text { Chloride }^{\mathrm{b}} \\
\text { (mg/L) }\end{array}$} & \multirow[b]{2}{*}{$\begin{array}{c}\text { Nitrite-plus- } \\
\text { nitrate } \\
(\mathrm{mg} \mathrm{N} / \mathrm{L})^{\mathrm{c}}\end{array}$} & \multirow[b]{2}{*}{$\begin{array}{c}\text { Total } \\
\text { nitrogen } \\
(\mathrm{mg} \mathrm{N} / \mathrm{L})^{\mathrm{d}}\end{array}$} & \multicolumn{2}{|c|}{ Method 1602-Single-agar layer } \\
\hline & & & & & & & $\begin{array}{c}\text { F-Specific } \\
\text { Coliphage } \\
\text { (USGS parameter } \\
\text { code 90904; } \\
\text { plaques per } \\
100 \mathrm{~mL} \text { ) } \\
\end{array}$ & $\begin{array}{c}\text { Somatic } \\
\text { Coliphage } \\
\text { (USGS parameter } \\
\text { code 90903; } \\
\text { plaques per } \\
100 \mathrm{~mL} \text { ) } \\
\end{array}$ & & & & & $\begin{array}{c}\text { F-Specific } \\
\text { Coliphage } \\
\text { (USGS parameter } \\
\text { code 90904; } \\
\text { plaques per } \\
100 \mathrm{~mL} \text { ) }\end{array}$ & $\begin{array}{c}\text { Somatic } \\
\text { Coliphage } \\
\text { (USGS parameter } \\
\text { code 90903; } \\
\text { plaques per } \\
100 \mathrm{~mL} \text { ) } \\
\end{array}$ \\
\hline \multirow[t]{3}{*}{ NITREX } & STE & 434203121311701 & 200305051000 & 45 & - & 51 & $<1$ & $<1$ & 200311190900 & - & - & - & $<1$ & $600^{\circ}$ \\
\hline & EOP & 434203121311204 & 200305051000 & 42 & - & 6.0 & $<1$ & $<1$ & 200311190900 & - & - & - & $<1$ & $<1$ \\
\hline & DFMW & 434203121311201 & 200305051000 & 9.8 & 3.6 & - & $<1$ & $<1$ & 200311190900 & - & - & - & $<1$ & $<1$ \\
\hline \multirow[t]{4}{*}{ NITREX } & STE & 433824121340601 & 200305190900 & 54 & - & 52 & $\mathrm{E} 7,400^{\mathrm{e}, \mathrm{h}}$ & 300 & 200311170800 & - & - & - & $4,400^{\circ}$ & $7,700^{\circ}$ \\
\hline & EOP & 433825121340004 & 200305190900 & 63 & - & 1.7 & $<1$ & $<1$ & 200311170800 & - & - & - & $<1$ & $<1$ \\
\hline & DFMW & 433825121340001 & 200305190900 & 7.0 & 1.4 & - & 2 & $<1$ & 200311170800 & - & - & - & $<1$ & $<1$ \\
\hline & $\begin{array}{l}\text { DFMW } \\
\text { resample }\end{array}$ & 433825121340001 & 200307140900 & - & - & - & $<1^{\mathrm{i}}$ & - & - & - & - & - & - & - \\
\hline \multirow[t]{3}{*}{ Puraflo } & STE & 434010121325602 & 200305190900 & 42 & - & 56 & E3, $900^{\mathrm{e}, \mathrm{h}}$ & 5 & 200311171100 & - & - & - & $67,000^{\mathrm{e}}$ & 510 \\
\hline & EOP & 434010121325604 & 200305190900 & 39 & - & 51 & 13 & $<1$ & 200311171100 & - & - & - & 250 & $<1$ \\
\hline & DFMW & 434010121325601 & 200305190900 & 7.0 & 1.2 & - & $<1$ & $<1$ & 200311171100 & - & - & - & $<1$ & $<1$ \\
\hline \multirow[t]{3}{*}{ Puraflo } & STE & 434324121292602 & 200306041000 & 48 & - & 74 & $6,400^{\mathrm{e}}$ & $<1$ & 200312011000 & 41.7 & - & - & 25 & $120,000^{\mathrm{e}}$ \\
\hline & EOP & 434324121292604 & 200306041000 & 43 & - & 82 & $<1$ & $<1$ & 200312011000 & 32.2 & - & - & $<1$ & $4,000^{\circ}$ \\
\hline & DFMW & 434324121292601 & 200306041000 & 44 & 57.4 & - & $<1$ & $<1$ & 200312011000 & 39.8 & - & - & $<1$ & $<1$ \\
\hline \multirow[t]{3}{*}{ Wert B } & STE & 434449121310202 & 200305141000 & 68 & - & 82 & 8 & 3 & 200311191100 & - & - & - & $<1$ & $12,000^{\mathrm{e}}$ \\
\hline & EOP & 434449121310204 & 200305141000 & 72 & - & 22 & $<1$ & 1 & 200311191100 & - & - & - & $<1$ & $25,000^{\mathrm{e}}$ \\
\hline & DFMW & 434449121310201 & 200305141000 & 8.2 & 6.1 & - & $<1$ & $<1$ & 200311191100 & - & - & - & $<1$ & $<1$ \\
\hline \multirow[t]{3}{*}{ Wert B } & STE & 434423121312902 & 200305141000 & 39 & - & 52 & $<1$ & 7 & 200311191000 & - & - & - & $340^{\circ}$ & $780,000^{\mathrm{e}}$ \\
\hline & EOP & 434423121312904 & 200305141000 & 35 & - & 14 & 8 & $<1$ & 200311191000 & - & - & - & $<1$ & $640,000^{\mathrm{e}}$ \\
\hline & DFMW & 434423121312901 & 200305141000 & 7.5 & 3.3 & - & $<1$ & $<1$ & 200311191000 & - & - & - & $<1$ & $<1$ \\
\hline \multirow[t]{3}{*}{ Dyno2 } & STE & 433950121322902 & 200305140900 & 240 & - & 110 & $37,000^{\mathrm{e}}$ & $34,000^{\mathrm{e}}$ & 200311171000 & - & - & - & $\mathrm{E} 220,000^{\mathrm{e}}$ & $5,800^{\circ}$ \\
\hline & EOP & 433950121322904 & 200305140900 & 210 & - & 48 & 760 & $23,000^{\mathrm{c}}$ & 200311171000 & - & - & - & 1,200 & 290 \\
\hline & DFMW & 433950121322901 & 200305140900 & 8.1 & 2.0 & - & $<1$ & $<1$ & 200311171000 & - & - & - & $<1$ & $<1$ \\
\hline
\end{tabular}


Table 12. Coliphage data for water from traditional and innovative onsite wastewater treatment systems, downgradient lysimeters, and downgradient ground water, La Pine, Oregon, 2003.-Continued

[Sample type: STE, septic tank effluent, EOP, end of pipe (end of innovative treatment), SFE, sand filter effluent, LYS, lysimeter, DFMW, drainfield monitoring well; date as year, month, day (YYYYMMDD); time in hours and minutes, military; $\mathrm{mg} / \mathrm{L}$, milligrams per liter; $\mathrm{mg} \mathrm{N} / \mathrm{L}$, milligrams nitrogen per liter; $\mathrm{mL}$, milliliter; total nitrogen data are provided for STE and EOP samples, whereas nitrite-plus-nitrate data are provided for SFE, LYS and DFMW samples because these oxidized nitrogen species are the dominant nitrogen species in these samples; "E", estimated; <, less than; >, greater than; --, no data]

\begin{tabular}{|c|c|c|c|c|c|c|c|c|c|c|c|c|c|c|}
\hline \multirow[b]{2}{*}{$\begin{array}{c}\text { Type of onsite } \\
\text { system }^{\mathrm{a}}\end{array}$} & \multirow[b]{2}{*}{$\begin{array}{c}\text { Sample } \\
\text { type }\end{array}$} & \multirow[b]{2}{*}{ Station number } & \multirow[b]{2}{*}{$\begin{array}{c}\text { Sample } \\
\text { date/time }\end{array}$} & \multirow[b]{2}{*}{$\begin{array}{l}\text { Chloride } \\
(\mathrm{mg} / \mathrm{L})^{b}\end{array}$} & \multirow[b]{2}{*}{$\begin{array}{c}\text { Nitrite-plus- } \\
\text { nitrate } \\
(\mathrm{mg} \mathrm{N} / \mathrm{L})^{\mathrm{c}}\end{array}$} & \multirow[b]{2}{*}{$\begin{array}{c}\text { Total nitrogen } \\
(\mathrm{mg} \mathrm{N} / \mathrm{L})^{\mathrm{d}}\end{array}$} & \multicolumn{2}{|c|}{ Method 1602-Single-agar layer } & \multirow[b]{2}{*}{$\begin{array}{c}\text { Sample } \\
\text { date/time }\end{array}$} & \multirow[b]{2}{*}{$\begin{array}{c}\text { Chloride }^{\mathrm{b}} \\
(\mathrm{mg} / \mathrm{L})\end{array}$} & \multirow[b]{2}{*}{$\begin{array}{c}\text { Nitrite-plus- } \\
\text { nitrate } \\
(\mathrm{mg} \mathrm{N} / \mathrm{L})^{\mathrm{c}}\end{array}$} & \multirow[b]{2}{*}{$\begin{array}{c}\text { Total } \\
\text { nitrogen } \\
(\mathrm{mg} \mathrm{N} / \mathrm{L})^{\mathrm{d}}\end{array}$} & \multicolumn{2}{|c|}{ Method 1602-Single-agar layer } \\
\hline & & & & & & & $\begin{array}{c}\text { F-Specific } \\
\text { Coliphage } \\
\text { (USGS paramete } \\
\text { code 90904; } \\
\text { plaques per } \\
100 \mathrm{~mL} \text { ) } \\
\end{array}$ & $\begin{array}{c}\text { Somatic } \\
\text { Coliphage } \\
\text { er (USGS parameter } \\
\text { code 90903; } \\
\text { plaques per } \\
100 \mathrm{~mL} \text { ) } \\
\end{array}$ & & & & & $\begin{array}{c}\text { F-Specific } \\
\text { Coliphage } \\
\text { (USGS parameter } \\
\text { code 90904; } \\
\text { plaques per } \\
100 \mathrm{~mL} \text { ) } \\
\end{array}$ & $\begin{array}{c}\text { Somatic } \\
\text { Coliphage (USGS } \\
\text { parameter code 90903; } \\
\text { plaques per } \\
100 \mathrm{~mL} \text { ) }\end{array}$ \\
\hline \multirow[t]{3}{*}{ Dyno2 } & STE & 434131121314302 & 200305050930 & 67 & - & 52 & $<1$ & $3,000,000^{\mathrm{e}}$ & 200311171200 & - & - & - & 1 & $8,900^{\circ}$ \\
\hline & EOP & 434131121314304 & 200305050930 & 39 & - & 20 & $<1$ & $290,000^{\mathrm{e}}$ & 200311171200 & - & - & - & $<1$ & 420 \\
\hline & DFMW & 434131121314301 & 200305050930 & 4.2 & 0.40 & - & $<1$ & $<1$ & 200311171200 & - & - & - & $<1$ & $<1$ \\
\hline
\end{tabular}

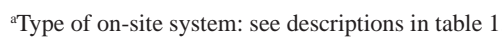

${ }^{b}$ Chloride analyzed by Oregon Department of Environmental Quality, except for December 2003 samples, which were analyzed by USGS.

'Nitrate-plus-nitrate analyzed by Oregon Department of Environmental Quality.

${ }^{\circledR}$ Total nitrogen analyzed by Oregon Department of Environmental Quality.

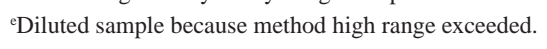

${ }^{\mathrm{f}}$ Also analyzed by method 1601, enrichment, presence/absence, USGS parameter code 99332; result: absent.

${ }^{8}$ Counts outside the acceptable range.

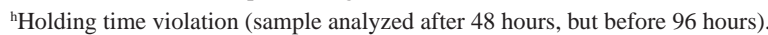

${ }^{i}$ Also analyzed by method 1601, enrichment, presence/absence, USGS parameter code 99335, result: absent. 
[Date, as year, month, day; time in hours and minutes, military; $\mathrm{mg} / \mathrm{L}$, milligrams per liter; $\mathrm{mg} \mathrm{N} / \mathrm{L}$, milligrams nitrogen per liter; $\mathrm{mL}$, milliliter; E, estimated;

$<$, less than; A, absent; P, present; --, no data]

\begin{tabular}{|c|c|c|c|c|c|c|c|c|}
\hline \multirow[b]{2}{*}{ Transect well name } & \multirow[b]{2}{*}{ Station number } & \multirow[b]{2}{*}{$\begin{array}{c}\text { Sample } \\
\text { date/time }\end{array}$} & \multirow[b]{2}{*}{$\begin{array}{c}\text { Chloride } \\
\text { (mg/L) }\end{array}$} & \multirow[b]{2}{*}{$\begin{array}{c}\text { Nitrite-plus- } \\
\text { nitrate } \\
\text { (mg N/L) } \\
\end{array}$} & \multicolumn{2}{|c|}{ Method 1602_Single-agar layer } & \multicolumn{2}{|c|}{$\begin{array}{l}\text { Method 1601—Enrichment, } \\
\text { presence/absence }\end{array}$} \\
\hline & & & & & $\begin{array}{c}\text { F-Specific } \\
\text { Coliphage } \\
\text { (USGS } \\
\text { parameter } \\
\text { code 90904; } \\
\text { plaques per } \\
100 \mathrm{~mL} \text { ) } \\
\end{array}$ & $\begin{array}{c}\text { Somatic } \\
\text { Coliphage } \\
\text { (USGS } \\
\text { parameter } \\
\text { code } 90903 ; \\
\text { plaques per } \\
100 \mathrm{~mL} \text { ) } \\
\end{array}$ & $\begin{array}{c}\text { F-Specific Coliphage } \\
\text { (USGS parameter } \\
\text { code 99335) }\end{array}$ & $\begin{array}{c}\text { Somatic Coliphage } \\
\text { (USGS parameter code } \\
\text { 99332) }\end{array}$ \\
\hline \multicolumn{9}{|c|}{ Senior Center } \\
\hline $\begin{array}{l}\text { Senior STE } \\
\text { ST }\end{array}$ & 434212121294299 & 200304301000 & - & - & E150,000,ab & $29,000^{\mathrm{a}}$ & - & - \\
\hline Senior 1 & 434212121294201 & 200304291100 & 5.22 & 1.94 & $<1$ & $<1$ & $\mathrm{~A}$ & A \\
\hline Senior 2 & 434212121294202 & 200304291300 & 6.26 & 4.48 & $<1$ & $<1$ & A & A \\
\hline Senior 3 & 434212121294203 & 200304301300 & 14.5 & 15.9 & $<1$ & $<1$ & A & A \\
\hline Senior 4 & 434212121294204 & 200304301600 & 5.71 & 4.79 & $<1$ & $<1$ & - & - \\
\hline Senior 5 & 434212121294205 & 200304301800 & 16.6 & 17.8 & $<1$ & $<1$ & - & - \\
\hline Senior 6 & 434212121294206 & 200305011000 & 6.4 & 4.82 & $<1$ & $<1$ & - & - \\
\hline Senior 7 & 434212121294207 & 200305011300 & 1.79 & 0.65 & $<1$ & $<1$ & - & - \\
\hline Senior 8 & 434212121294208 & 200305011500 & 12.6 & 13.4 & $<1$ & $<1$ & - & - \\
\hline Senior 9 & 434212121294209 & 200305011700 & 1.09 & 0.11 & 5 & $<1$ & - & - \\
\hline Senior 10 & 434212121294210 & 200305021100 & 3.30 & 1.55 & 3 & $<1$ & - & - \\
\hline Senior 11 & 434212121294211 & 200305021200 & 1.78 & 0.27 & $<1$ & $<1$ & 一 & 一 \\
\hline 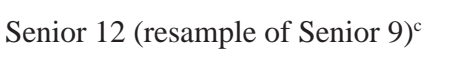 & 434212121294212 & 200306181400 & - & - & $<1$ & $<1$ & A & A \\
\hline Senior 13 (resample of Senior 10) ${ }^{\mathrm{d}}$ & 434212121294213 & 200306181700 & - & - & $<1$ & $<1$ & A & A \\
\hline Senior 14 (resample of Senior 10) ${ }^{\mathrm{d}}$ & 434212121294214 & 200306181800 & - & - & $<1$ & $<1$ & A & A \\
\hline
\end{tabular}


[Date, as year, month, day; time in hours and minutes, military; mg/L, milligrams per liter; mg N/L, milligrams nitrogen per liter; $\mathrm{mL}$, milliliter; E, estimated;

$<$, less than; A, absent; P, present; --, no data]

\begin{tabular}{|c|c|c|c|c|c|c|c|c|}
\hline \multirow[b]{2}{*}{ Transect well name } & \multirow[b]{2}{*}{ Station number } & \multirow[b]{2}{*}{$\begin{array}{c}\text { Sample } \\
\text { date/time }\end{array}$} & \multirow[b]{2}{*}{$\begin{array}{c}\text { Chloride } \\
\text { (mg/L) }\end{array}$} & \multirow[b]{2}{*}{$\begin{array}{c}\text { Nitrite-plus- } \\
\text { nitrate } \\
\text { (mg N/L) }\end{array}$} & \multicolumn{2}{|c|}{ Method 1602-Single-agar layer } & \multicolumn{2}{|c|}{$\begin{array}{c}\text { Method } 1601-\text { Enrichment, } \\
\text { presence/absence }\end{array}$} \\
\hline & & & & & $\begin{array}{c}\text { F-Specific } \\
\text { Coliphage } \\
\text { (USGS parameter } \\
\text { code 90904; } \\
\text { plaques per } \\
100 \mathrm{~mL} \text { ) } \\
\end{array}$ & $\begin{array}{c}\text { Somatic } \\
\text { Coliphage } \\
\text { (USGS parameter } \\
\text { code 90903; } \\
\text { plaques per } \\
100 \mathrm{~mL} \text { ) } \\
\end{array}$ & $\begin{array}{c}\text { F-Specific Coliphage } \\
\text { (USGS parameter } \\
\text { code 99335) }\end{array}$ & $\begin{array}{c}\text { Somatic Coliphage } \\
\text { (USGS parameter code } \\
\text { 99332) }\end{array}$ \\
\hline \multicolumn{9}{|c|}{ High Lakes Church } \\
\hline High Lakes 1 & 434241121311601 & 200306101600 & 3.12 & 0.49 & $<1$ & $<1$ & $\mathrm{~A}$ & $\mathrm{~A}$ \\
\hline High Lakes 2 & 434241121311602 & 200306101800 & 26.3 & 19.0 & $<1$ & $<1$ & A & A \\
\hline High Lakes 3 & 434241121311603 & 200306111200 & 14.0 & $<0.02$ & $<1$ & $<1$ & A & A \\
\hline High Lakes 4 & 434241121311604 & 200306111500 & 4.78 & 0.29 & $<1$ & $<1$ & A & A \\
\hline High Lakes 5 & 434241121311605 & 200306111600 & 7.92 & 3.57 & $<1$ & $<1$ & A & A \\
\hline High Lakes 6 & 434241121311606 & 200306121300 & 19.6 & 10.6 & $<1$ & $<1$ & A & A \\
\hline High Lakes 7 & 434241121311607 & 200306121500 & 19.6 & 10.3 & $<1$ & $<1$ & A & $\mathrm{A}$ \\
\hline High Lakes 8 & 434241121311608 & 200306121900 & 1.73 & 0.72 & $<1$ & $<1$ & A & A \\
\hline High Lakes 9 & 434241121311609 & 200306131100 & 1.98 & 0.11 & $<1$ & $<1$ & A & A \\
\hline \multicolumn{9}{|c|}{ Pine Forest Road } \\
\hline Pine 1 & 434210121313401 & 200306091200 & 51.0 & 35.5 & $<1$ & $<1$ & A & A \\
\hline Pine 2 & 434210121313402 & 200306091400 & 8.18 & 4.80 & $<1$ & $<1$ & $\mathrm{~A}$ & A \\
\hline Pine 3 & 434210121313403 & 200306091600 & 10.1 & 5.86 & $<1$ & $<1$ & A & A \\
\hline Pine 4 & 434210121313404 & 200306091800 & 37.0 & 25.6 & $<1$ & $<1$ & A & A \\
\hline Pine 5 & 434210121313405 & 200306161300 & 4.44 & 2.03 & $<1$ & $<1$ & A & $\mathrm{P}$ \\
\hline Pine 6 & 434210121313406 & 200306161500 & 10.4 & 6.22 & $<1$ & $<1$ & A & $\mathrm{A}$ \\
\hline Pine 7 & 434210121313407 & $20030617 \quad 1100$ & 5.03 & 2.63 & $<1$ & $<1$ & A & A \\
\hline
\end{tabular}


[Date as year, month, day (YYYYMMDD); time in hours and minutes, military; mg/L, milligrams per liter; mg N/L, milligrams nitrogen per liter; mL, milliliter; E, estimated;

$<$, less than; A, absent; P, present; --, no data]

\begin{tabular}{|c|c|c|c|c|c|c|c|c|}
\hline \multirow[b]{2}{*}{ Transect well name } & \multirow[b]{2}{*}{ Station number } & \multirow[b]{2}{*}{$\begin{array}{c}\text { Sample } \\
\text { date/time }\end{array}$} & \multirow[b]{2}{*}{$\begin{array}{c}\text { Chloride } \\
\text { (mg/L) }\end{array}$} & \multirow[b]{2}{*}{$\begin{array}{c}\text { Nitrite-plus- } \\
\text { nitrate } \\
\text { (mg N/L) }\end{array}$} & \multicolumn{2}{|c|}{ Method 1602-Single-agar layer } & \multicolumn{2}{|c|}{$\begin{array}{c}\text { Method } 1601-E n r i c h m e n t, \\
\text { presence/absence }\end{array}$} \\
\hline & & & & & $\begin{array}{c}\text { F-Specific } \\
\text { Coliphage } \\
\text { (USGS parameter } \\
\text { code 90904; } \\
\text { plaques per } \\
100 \mathrm{~mL} \text { ) } \\
\end{array}$ & $\begin{array}{c}\text { Somatic } \\
\text { Coliphage } \\
\text { (USGS parameter } \\
\text { code 90903; } \\
\text { plaques per } \\
100 \mathrm{~mL} \text { ) }\end{array}$ & $\begin{array}{c}\text { F-Specific Coliphage } \\
\text { (USGS parameter } \\
\text { code 99335) }\end{array}$ & $\begin{array}{c}\text { Somatic Coliphage } \\
\text { (USGS parameter code } \\
\text { 99332) }\end{array}$ \\
\hline \multicolumn{9}{|c|}{ Pine Forest Road_-Continued } \\
\hline$\overline{\text { Pine } 8}$ & 434210121313408 & $20030617 \quad 1300$ & 12.9 & 7.63 & $<1$ & $<1$ & $\mathrm{~A}$ & A \\
\hline Pine 9 & 434210121313409 & $20030617 \quad 1500$ & 18.3 & 12.4 & $<1$ & $<1$ & A & A \\
\hline Pine 10 & 434210121313410 & 200306191000 & 10.6 & 5.19 & 1 & $<1$ & $\mathrm{~A}$ & A \\
\hline Pine 11 & 434210121313411 & 200306191200 & 39.8 & 30.4 & $<1$ & $<1$ & A & $\mathrm{A}$ \\
\hline
\end{tabular}

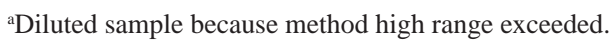

${ }^{\mathrm{b}}$ Counts outside the acceptable range.

'Senior 12 drilled to resample the original screened interval of Senior 9.

dSenior 13 and Senior 14 drilled to different depths to resample the original screened interval of Senior 10. 


\section{Coliphage in Onsite Wastewater}

Innovative system network samples collected from septic tanks ( 26 of the 28 systems, because the two Amphidrome systems were not instrumented for septic tank access) provide a measure of the magnitude and variability of coliphage concentrations in onsite wastewater. The distributions of concentrations (fig. 6) are shown for F-specific and somatic coliphage, both for the group of all samples (26 systems, 2 sampling events), and for the subset (16 systems, 2 sampling events) of onsite wastewater treatment systems without recirculation (recirculation back to the septic tank was a part of the treatment process in some types of innovative systems, as described in table 1). Coliphage concentrations varied by orders of magnitude, with F-specific coliphage concentrations ranging from $<1$ to $270,000 \mathrm{PFU} / 100 \mathrm{~mL}$, and somatic coliphage concentrations ranging from $<1$ to $3,000,000 \mathrm{PFU} / 100$ $\mathrm{mL}$. The variability in coliphage concentrations in onsite wastewater probably reflects variability in coliphage concentrations among different individuals in the human population and variability in coliphage concentrations within individual people over time. Variability in coliphage concentrations among different onsite wastewater treatment systems might also reflect environmental differences among onsite wastewater treatment systems (e.g., variations in temperature, presence or absence of chemicals harmful to coliphage, different residence times among systems, etc.). The orders-of-magnitude variability in coliphage concentrations in onsite wastewater is greater than that reported for typical municipal wastewater (concentrations typically ranging from 100 to $1,000 \mathrm{PFU} / 100$ $\mathrm{mL}$; Gerba, 2000b, p. 492). Lower variability of coliphage concentrations in municipal wastewater may reflect the homogenizing effect inherent in municipal wastewater systems, where mixing of human wastewater from large numbers of individuals smoothes out individual variability.

The innovative onsite wastewater treatment systems sampled as part of this project were not designed to remove coliphage. However, because innovative systems were sampled for coliphage both at the septic tank and at end-of-pipe, these paired samples allow estimation of the degree of coliphage attenuation within innovative onsite wastewater treatment systems. Attenuation can be represented simply by the endof-pipe coliphage concentration divided by the septic tank coliphage concentration; this quotient, presented as a fraction or percent, represents the amount remaining after treatment, normalized by the input concentration. Variability in coliphage concentrations in onsite wastewater treatment systems over time will, of course, create uncertainty in these calculations. For example, if an unusually low concentration of coliphage is present in the septic tank at the time of sampling, or if an unusually high concentration of coliphage is present in the end-of-pipe sample due to a slug of coliphage-rich wastewater having been processed through the innovative onsite wastewater treatment system, then the quotient may indicate an apparent increase in coliphage concentrations. A nonparametric measure of attenuation, such as the median fraction remaining

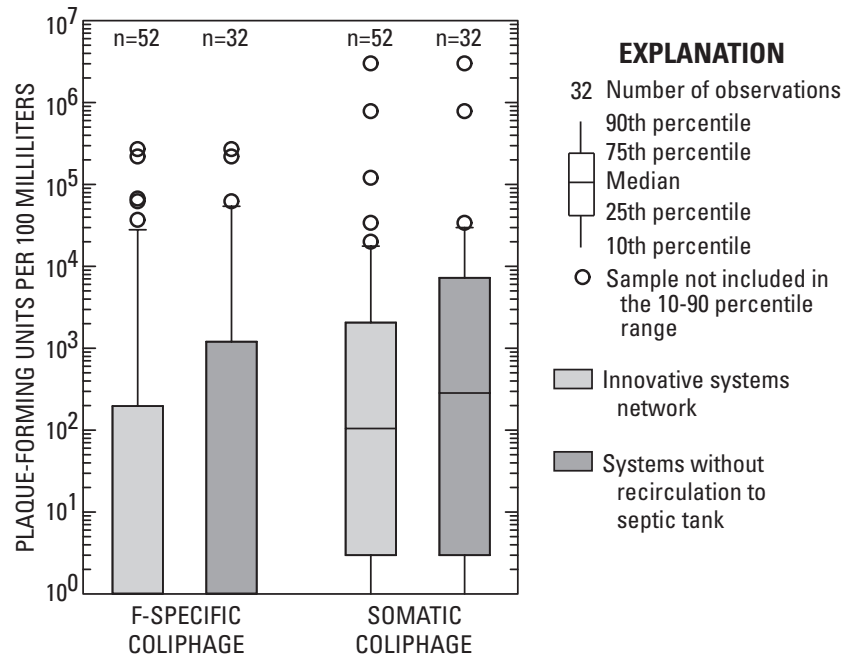

Figure 6. Statistical distribution of coliphage concentrations in septic tank effluent samples from innovative systems network near La Pine, Oregon, 2003. (Portions of boxes and [or] lower whiskers extending below the laboratory reporting level of 1 PFU [plaque-forming units] / $100 \mathrm{~mL}$ [milliliters] are censored at the laboratory reporting level, such that, for F-specific coliphage, medians are $<1 \mathrm{PFU} / 100 \mathrm{~mL}$, and for somatic coliphage, 10th percentiles are $<1 \mathrm{PFU} / 100 \mathrm{~mL}$. One value for somatic coliphage, reported as $>1,000 \mathrm{PFU} / 100 \mathrm{~mL}$ plotted at 1,000 PFU/100 mL.)

after treatment, will be less likely to be affected by temporal variability in coliphage concentrations than will parametric measures such as the mean fraction remaining. Thus, median fraction remaining after treatment is reported here. For this calculation, paired septic tank/end-of-pipe samples were evaluated. Standard, pressure, and sand filter systems were not evaluated, and innovative onsite wastewater treatment systems with recirculation to the septic tank were not evaluated. Data from sites at which both the septic tank and the end-of-pipe samples were reported as less than the method detection limit were not used. The results were: F-specific coliphage, $\leq 0.71$ ( $\leq 71$ percent remaining after treatment) $(\mathrm{n}=15)$, and somatic coliphage, $\leq 0.12$ ( $\leq 12$ percent remaining after treatment) $(n=18)$. (Censored values for the fraction remaining occurred because some of the reported coliphage concentrations were censored, usually as $<1 \mathrm{PFU} / 100 \mathrm{~mL}$.)

Some coliphage attenuation appeared to occur during the processing of wastewater in innovative onsite wastewater treatment systems. The greater apparent loss of somatic coliphage compared with F-specific coliphage may be related to the reactivity of these different bacteriophage, in so far as F-specific coliphage are more resistant to inactivation than are somatic coliphage (IAWPRC Study Group on Health Related Water Microbiology, 1991). However, treatment of water for pathogen removal is typically gauged in terms of orders-ofmagnitude reduction, and thus it is not clear if the observed 
reductions are meaningful from a management standpoint. On the other hand, the apparent reductions were a byproduct of treatment of wastewater primarily for nitrogen, biological oxygen demand, total suspended solids, and the indicator bacteria fecal coliform and E. coli, and thus they represent, to the degree that the estimated reductions represent actual reductions, an unintended improvement in onsite wastewater quality.

The hypothesis that coliphage loss during innovative treatment might occur can be evaluated empirically by comparing septic tank coliphage concentrations for the group of sites without recirculation with concentrations for all sites (fig. 6). Higher overall concentrations of both F-specific and somatic coliphage in the group of systems without recirculation, compared with the group of all systems including those with recirculation, may reflect some coliphage loss during treatment and recirculation. However, such cause-and-effect was not demonstrated in a controlled study.

\section{Coliphage in Lysimeter Samples}

Coliphage samples were collected from five lysimeters, at two different times each (table 12). Of the five sites, four were standard or pressure systems, and one was an innovative system. Thus, septic tank samples represent source samples for standard and pressure system lysimeter samples, and end-of-pipe samples represent source samples for the innovative system lysimeter samples. Concentrations of coliphage in lysimeter samples were generally lower than those in source samples. These relations also held when lysimeter and source coliphage concentrations were normalized to chloride concentrations to account for dilution with rain and snowmelt and for enrichment from evapotranspiration. Attenuation is not reported because the number of paired samples available for such analysis was small.

\section{Coliphage in Drainfield Monitoring Well and Transect Samples}

Drainfield monitoring wells downgradient from onsite wastewater treatment systems were sampled and the water analyzed for coliphage to provide information about the occurrence of coliphage in ground water, and transect wells installed along plumes of onsite wastewater were sampled and the water analyzed for coliphage to provide information on coliphage occurrence and transport in ground water. The two networks of wells, the innovative system network and the transect wells, were installed close to sources of onsite wastewater. The elevated concentrations of chloride and nitrate in most samples from the transect well networks indicates that the goal of sampling wells that intercepted plumes of onsite wastewater was achieved. Coliphage were rarely detected in these groundwater samples, and none of the detections were reproducible.

There was no general sense of what range of coliphage concentrations might be present in the ground-water samples prior to analysis of the first sets of samples. Coliphage were not commonly detected in the ground-water samples. The possibility that the few reported detections might be false positives representing field or laboratory contamination was considered. To better understand the occurrence of coliphage in ground water, detections of coliphage in ground-water samples were followed up by resampling. Resampled innovative system network drainfield monitoring well samples were analyzed for coliphage by both the quantitative method and the enrichment method. In all four instances where coliphage were detected in innovative system network drainfield monitoring well samples (one instance of F-specific coliphage at $2 \mathrm{PFU} / 100 \mathrm{~mL}$; three instances of somatic coliphage at 1 to $8 \mathrm{PFU} / 100 \mathrm{~mL}$ ), no coliphage were detected by either the quantitative or the enrichment methods upon resampling. Two transect wells, Senior 9 and 10, had reported detections of coliphage (F-specific coliphage, 3 to 5 PFU/100 mL). Transect wells were temporary direct-push wells, so resampling of wells Senior 9 and 10 required redrilling. Redrilling and setting the same screened interval as that of well Senior 9 was accomplished with one new well, whereas well Senior 10 required two new wells to capture the same screened interval as the original. Again, no coliphage were detected by either the quantitative or the enrichment methods upon resampling. To minimize the potential need for redrilling, ground water from the next two transects that were installed, the High Lakes and the Pine transects, was sampled in duplicate, and analyzed by both the quantitative and the enrichment methods. The sample from well Pine 05 was reported to contain somatic coliphage with analysis by enrichment, but coliphage were not detected with analysis by the quantitative method. The sample from well Pine 10 was reported to contain F-specific coliphage with analysis by the quantitative method $(1 \mathrm{PFU} / 100 \mathrm{~mL})$, but coliphage were not detected with analysis by the enrichment method. Thus, none of the eight reported detections were reproduced.

We interpret the consistent lack of reproducibility of detections to indicate that the detections reported for groundwater samples represented low-level field or laboratory contamination. This interpretation leads to the conclusion that coliphage were effectively attenuated to less than the quantitative-method method detection limit of 1 PFU/100 $\mathrm{mL}$ in the sediments of the La Pine aquifer (or the unsaturated zone above it) and for the distances represented by the observation networks. It is unlikely that low-level field or laboratory coliphage contamination had a substantial effect on coliphage results from onsite wastewater samples, because onsite wastewater samples frequently contained coliphage at concentrations orders of magnitude greater than the apparent low-level field or laboratory contamination levels. In contrast, low-level field or laboratory coliphage contamination clearly has the potential to significantly affect coliphage results from ground-water samples. Although detections in the groundwater samples may have been a result of contamination during sampling or analysis, alternative interpretations of these coliphage data could be proposed. For example, temporally variable coliphage occurrence at individual sampling sites 
could reflect the difficulty of measuring coliphage at concentrations near method detection limits, or temporally variable transport that can occur in response to geochemical perturbations such as changing ground-water $\mathrm{pH}$ (Bales and others, 1997). However, the complete absence of coliphage detections in the La Pine confirmatory (replicate and repeat) samples suggests that these alternative explanations are unlikely for this data set. Furthermore, the coliphage detection at Senior 9 is suspicious because other indicators of onsite wastewater were essentially absent: the chloride concentration was low $(1.09 \mathrm{mg} / \mathrm{L})$, as was the nitrate concentration $(0.11 \mathrm{mg} / \mathrm{L}$ in well-oxygenated ground water, water that would not favor denitrification). Lastly, the temporal distribution of some of the reported coliphage detections for ground-water samples suggests the possibility of some sort of systematic contamination effect, as explained next. Of the four innovative systems network drainfield monitoring well samples with coliphage detections, three were collected and shipped on the same day (April 23, 2003). Similarly, the two Senior transect samples with coliphage detections, although collected on different days (5 PM on May 1, 2003, and 11 AM on May 2, 2003), were shipped to the analytical laboratory on the same day (May 2, 2003). These observations do not, though, confirm the presence of a systematic contamination effect, and no explanatory contamination process has been identified.

The apparent absence of coliphage transport along the transects stands in contrast to the results of DeBorde and others (1998), who also evaluated transport of onsite-wastewater-derived coliphage in a field setting using a plume-scale transect design linking a series of monitoring wells to a known source. DeBorde and others (1998) observed transport of onsite-wastewater-derived coliphage over a distance of tens of feet in a coarse-grained alluvial aquifer in Montana. An understanding of conditions or processes that facilitated coliphage transport at the Montana site, but that favored attenuation at the La Pine sites, could provide a basis for a more general understanding aquifer vulnerability to coliphage. Coliphage transport and fate may be controlled by variations in aquifer lithology (which affect both sorption reactions and groundwater velocities), onsite wastewater treatment system water fluxes (which affect advection, and also may affect sorption), and (or) variations in ambient geochemical conditions (which control sorption and inactivation rates). The aquifer studied by DeBorde and others (1998) was coarse-grained (sand and gravel), whereas the La Pine sites were composed primarily of sand (table 5). Although both sites received recharge from onsite wastewater treatment systems, the Montana site received discharge from a system serving a public school, whereas the La Pine sites received discharge from onsite wastewater treatment systems serving individual homes, one rural church, and one small (day-use only) senior center-discharges that may have been lower on an area-weighted basis than those associated with the public school. Ambient $\mathrm{pH}$ and temperature, however, were similar among the two field sites (circumneutral $\mathrm{pH}$, temperatures generally in the range of 9 to $\left.12^{\circ} \mathrm{C}\right)$. As has been pointed out by IAWPRC Study Group on Health Related Water Microbiology (1991), studies of virus transport using naturally occurring viruses are few. Systematic studies such as this one and the work by DeBorde and others (1998) are beginning to form a transect-based foundation from which an improved understanding of field conditions and processes promoting virus transport or fate may eventually be generated.

\section{Summary and Conclusions}

Organic wastewater compounds were frequently detected in onsite wastewater; concentrations commonly were on the order of tens of micrograms per liter. Organic wastewater compounds also were detected in ground water, but less frequently, and detections were mostly at concentrations below $1 \mu \mathrm{g} / \mathrm{L}$. Organic wastewater compound concentrations, normalized to chloride concentrations, generally decreased from onsite wastewater treatment systems to downgradient ground water. Eight organic wastewater compounds were detected in the 20 ground water samples associated with the innovative systems network (detection frequencies up to 30 percent), and 6 different organic wastewater compounds were detected in the 31 ground-water samples associated with transect wells emplaced along ground-water flowpaths downgradient from onsite wastewater treatment system drainfield lines (detection frequencies up to 16 percent).

Ground-water samples from one transect were analyzed for pharmaceuticals. Sulfamethoxazole (an antibacterial), acetaminophen (an analgesic), and caffeine (a stimulant, but not a medical drug) each were detected once. In addition, the anticonvulsant drugs primidone and phenobarbitol were tentatively identified in three ground-water samples from one nest of wells at a separate transect.

The shallow aquifer from which the ground-water samples were collected is the primary source of water for most residents of the La Pine region. The effects of microgramper-liter or sub-microgram-per-liter concentrations of organic wastewater compounds and (or) pharmaceuticals consumed over long periods of time are largely unknown, as are the additive or synergistic effects associated with exposure to combinations of multiple organic wastewater compounds and (or) pharmaceuticals. Some organic wastewater compounds are thought to have endocrine-disrupting properties, and pharmaceuticals are designed to impart biological effects in animals. There also is the potential for some organic wastewater compounds or pharmaceuticals to eventually discharge to streams, where the effects on aquatic organisms are largely unknown.

Dispersion and attenuation of organic wastewater compounds may explain the low concentrations observed in ground-water samples. However, although organic wastewater compounds were detected more frequently in groundwater samples with larger components of onsite wastewater (as inferred by chloride concentrations), and, in the case of 
transect wells, in ground-water samples proximal to onsite wastewater treatment system drainfield lines, overall occurrence patterns exhibited great variability. Organic wastewater compound occurrence and transport might be significantly affected by temporal variability of organic wastewater compound concentrations in onsite wastewater sources. Nitrogen and chloride concentrations in onsite wastewater exhibited small variability among systems, but concentrations of individual organic wastewater compounds among different onsite wastewater treatment systems varied dramatically- not uncommonly by several orders of magnitude. Thus, although temporal variability of organic wastewater compound concentrations in individual onsite wastewater treatment systems was not characterized in this study, the variability among onsite wastewater treatment systems suggest that loading of some organic wastewater compounds to the environment from individual onsite wastewater treatment systems over time might also be highly variable. Highly variable source terms likely would not be as amenable to transport modeling as would be more uniform loading such as might be expected for onsite-wastewater-derived nitrate and chloride. Similar patterns of variable pharmaceutical occurrence in ground water beg questions regarding variability in pharmaceutical loading to the environment from onsite wastewater treatment systems. For example, do some of the occasional detections of pharmaceuticals in ground water represent relatively uniform upgradient inputs, with variable degrees of dilution and attenuation? Do some represent only occasional upgradient use of pharmaceuticals? Do some represent upgradient loading from episodic disposal that might occur when users of onsite wastewater treatment systems dispose of old pharmaceuticals by the common method of flushing down the toilet? Comparable questions could be composed for organic wastewater compounds. Characterization of the temporal variability of source strength may become one of the critical challenges in organic wastewater compound and pharmaceutical transport studies. However, the hypothesis that variability in organic wastewater compound and pharmaceutical loading might be important for understanding transport should not detract from the fact that many of these compounds are reactive, and that detailed understanding of sorption and degradation of these compounds will be essential to any transport work.

Coliphage were frequently detected in onsite wastewater, occasionally detected in lysimeters, but only sporadically detected in samples from wells located adjacent to or under onsite wastewater treatment system drainfield lines (detected in eight ground-water samples, but below method detection limits in all eight replicate or repeat samples). Coliphage concentrations in onsite wastewater varied by orders of magnitude, with $\mathrm{F}$-specific coliphage concentrations ranging from $<1$ to $270,000 \mathrm{PFU} / 100 \mathrm{~mL}$, and somatic coliphage concentrations ranging from $<1$ to $3,000,000 \mathrm{PFU} / 100 \mathrm{~mL}$. The variability in coliphage concentrations observed in onsite wastewater is greater than that typically reported for municipal wastewater. The consistent absence of coliphage detections in the La Pine confirmatory (replicate and repeat) ground-water samples is interpreted to indicate that the detections reported for ground-water samples represented low-level field or laboratory contamination, and we suggest that coliphage were effectively attenuated to less than $1 \mathrm{PFU} / 100 \mathrm{~mL}$ over distances of less than several feet of transport in the unsaturated zone and (or) aquifer.

If coliphage survival and transport are representative of enteric virus survival and transport, the apparent absence of detectable concentrations of coliphage in the sand aquifer of La Pine might be construed positively by users of that resource. However, broader-based understanding of aquifer vulnerability to virus survival and transport remains elusive. Few plume-scale studies of naturally occurring viruses from onsite wastewater treatment systems in relatively undisturbed, natural settings have been undertaken, and results to date raise questions about factors controlling aquifer vulnerability to virus survival and transport. An understanding of conditions or processes that facilitate coliphage transport in some environments, but attenuation in others, could provide a basis for a more general understanding of field conditions and processes controlling aquifer vulnerability to coliphage.

\section{Acknowledgments}

This project was a cooperative effort by USGS, ODEQ, and DCEHD, with active participation by personnel from ODEQ and DCEHD. The project benefited from close collaboration by numerous individuals. The staff at the La Pine Senior Center and the High Lakes Christian Church granted permission for the emplacement of 23 temporary monitoring wells on the 2 properties. Dan Haldeman and Todd Cleveland (DCEHD) provided key communications links between the USGS and owners of property where monitoring occurred, orchestrated many aspects of field efforts for monitoring of onsite wastewater treatment systems, and were a wealth of technical information. Dave Anderson, Tracy England, Jim Glass, Don Hanson, Mark Pugh and Bob Williams (ODEQ) provided crucial drilling support. Rebecca Bushon and Donna Francy (USGS) accommodated our need to drill 5 days a week by accepting weekend delivery of coliphage samples at their homes, transporting them to the laboratory facilities, and analyzing them immediately.

\section{References Cited}

Agency for Toxic Substances and Disease Registry, 2004, ToxFAQs for cresols, retrieved April 28, 2005 from http:// www.atsdr.cdc.gov/tfacts34.html.

Bales, R.C., Li, Shimin, Maguire, K.M., Yahya, M.T., Gerba, C.P., and Harvey, R.W., 1995, Virus and bacteria transport in a sandy aquifer, Cape Cod, MA: Ground Water, v. 33, p. 653-661. 
Bales, R.C., Li, Shimin, Yeh, T.C.J., Lenczewski, M.E., and Gerba, C.P., 1997, Bacteriophage and microsphere transport in saturated porous media: Forced-gradient experiment at Borden, Ontario: Water Resources Research, v. 33, p. 639-648.

Barnes, K.K., Christenson, S.C., Kolpin, D.W., Focazio, M.J., Furlong, E.T., Zaugg, S.D., Meyer, M.T., and Barber, L.B., 2004, Pharmaceuticals and other organic waste water contaminants within a leachate plume downgradient of a municipal landfill: Ground Water Monitoring and Remediation, v. 24, p. 119-126.

Century West Engineering Corporation, 1982, La Pine Aquifer Management Plan, Bend, Oregon, variously paginated.

Childress, C.J.O., Foreman, W.T., Connor, B.F., and Maloney, T.J., 1999, New reporting procedures based on longterm method detection levels and some considerations for interpretations of water-quality data provided by the U.S. Geological Survey National Water Quality Laboratory: U.S. Geological Survey Open-File Report 99-193, 19 p.

Clesceri, L.S., Greenberg, A.E., and Eaton, A.D., eds., 1998, Standard Methods for the Examination of Water and Wastewater, 20th ed.: Washington, D.C., American Public Health Association, variously paginated.

Daughton, C.G., 2001, Pharmaceuticals and personal care products in the environment, overarching issues and overview: in Daughton, C.G., and Jones-Lepp, T.L., eds., Pharmaceuticals and personal care products in the environment: Scientific and regulatory issues: Washington, D.C., American Chemical Society, p. 2-38.

Daughton, C.G., and Ternes, T.A., 1999, Pharmaceuticals and personal care products in the environment: Agents of subtle change?: Environmental Health Perspectives, v. 107, Supplement 6, p. 907-942.

DeBorde, D.C., Woessner, W.W., Lauerman, B., and Ball, P.N., 1998, Virus occurrence and transport in a school septic system and unconfined aquifer: Ground Water, v. 36, p. $825-834$.

Deschutes County Environmental Health Division, no date, La Pine National Demonstration Project, http://marx.deschutes. org/deq/lapineindex.htm.

Drewes, J.E., Heberer, T., Rauch, T., and Reddersen, K., 2003, Fate of pharmaceuticals during ground water recharge: Ground Water Monitoring and Remediation, v. 23, p. 64-72.

Fishman, M.J., ed., 1993, Methods of analysis by the U.S. Geological Survey National Water Quality LaboratoryDetermination of inorganic and organic constituents in water and fluvial sediments: U.S. Geological Survey OpenFile Report 93-125, 217 p.
Francy, D.S., Myers, D.N., and Helsel, D.R., 2000, Microbiological monitoring for the U.S. Geological Survey National Water-Quality Assessment Program: U.S. Geological Survey Water-Resources Investigations Report 00-4018, 31 p.

Gannett, M.W., Lite, K.E., Jr., Morgan, D.S., and Collins, C.A., 2001, Ground-water hydrology of the Upper Deschutes Basin, Oregon: U.S. Geological Survey WaterResources Investigations Report 00-4162, 77 p.

Gerba, C.P., 2000a, Domestic wastes and waste treatment, in Maier, R.M., Pepper, I.L., and Gerba, C.P., eds.: San Diego, Environmental microbiology, Academic Press, p. 505-534.

Gerba, C.P., 2000b, Indicator microorganisms, in Maier, R.M., Pepper, I.L., and Gerba, C.P., eds., Environmental microbiology, San Diego, Academic Press, p. 491-503.

Guy, H.P., 1969, Laboratory theory and methods for sediment analysis: U.S. Geological Survey Techniques of WaterResources Investigations, Book 5, Chap. C1, 58 p.

Handzel, T.R., Green, R.M., Sanchez, C., Chung, H., and Sobsey, M.D., 1993, Improved specificity in detecting F-specific coliphages in environmental samples by suppression of somatic phages: Water Science and Technology, v. 27, p. $123-131$.

Helsel, D.R., and Hirsch, R.M., 1992, Statistical methods in water resources: Amsterdam, The Netherlands, Elsevier, $522 \mathrm{p}$.

Hinkle, S.R., Böhlke, J.K., Duff, J.H., Morgan, D.S., and Weick, R.J., 2002, Nitrate source, transport and fate in ground water near La Pine, Oregon (abstract), Eos, Transactions of the American Geophysical Union, 83, fall meeting supplement.

IAWPRC Study Group on Health Related Water Microbiology, 1991, Bacteriophages as model viruses in water quality control: Water Research, v. 25, p. 529-545.

Keswick, B.H., and Gerba, C.P., 1980, Viruses in ground water: Environmental Science Technology, v. 14, p. 12901297.

Kolpin, D.W., Furlong, E.T., Meyer, M.T., Thurman, E.M., Zaugg, S.D., Barber, L.B., and Buxton, H.T., 2002, Pharmaceuticals, hormones, and other organic wastewater contaminants in U.S. streams, 1999-2000: A national reconnaissance: Environmental Science and Technology, v. 36, p. 1202-1211.

Lite, K.E., Jr., and Gannett, M.W., 2002, Geologic framework of the regional ground-water flow system in the upper Deschutes Basin, Oregon: U.S. Geological Survey WaterResources Investigations Report 02-4015, 44 p. 
Masters, R.W., Verstraeten, I.M., and Heberer, Thomas, 2004, Fate and transport of pharmaceuticals and endocrine disrupting compounds during ground water recharge: Ground Water Monitoring and Remediation, v. 24, p. 54-57.

Morgan, D.S., Hinkle, S.R., and Weick, R.J., 2002, Simulation of flow and transport of septic-derived nitrate at multiple scales within a heterogeneous alluvial aquifer system (abstract), Eos, Transactions of the American Geophysical Union, 83, fall meeting supplement.

National Atmospheric Deposition Program, no date, Statistical summary of precipitation chemistry for valid samples, retrieved February 26, 2004 from http://nadp.sws.uiuc.edu/.

Oregon Department of Environmental Quality, 1994, Statewide Groundwater Monitoring Program, Lapine Area Groundwater Investigation, Deschutes County, Oregon, volume II: Portland, Oregon, variously paginated.

Oregon Department of Environmental Quality, 2004, La Pine Demonstration Project, http://www.deq.state.or.us/wq/ onsite/LaPine.htm.

Seiler, R.L., Zaugg, S.D., Thomas, J.M., and Howcroft, D.L., 1999, Caffeine and pharmaceuticals as indicators of waste water contamination in wells: Ground Water, v. 37, p. 405-410.

Sidki, A.M., Smith, D.S., and Landon, J., 1985, Dual-label fluoroimmunoassay for simultaneous determination of primidone and phenobarbital: Therapeutic Drug Monitoring, v. 7, p. 101-107.

Snyder, S.A., Kelly, K.L., Grange, A.H., Sovocool, G.W., Snyder, E.M., and Giesy, J.P., 2001, Pharmaceuticals and personal care products in the waters of Lake Mead, Nevada, in Daughton, C.G., and Jones-Lepp, T.L., eds., Pharmaceuticals and personal care products in the environment, Scientific and regulatory issues: Washington, D.C., American Chemical Society, p. 116-138.

Taggart, J.E., Jr., ed., 2002, Analytical methods for chemical analysis of geologic and other materials, U.S. Geological Survey: U.S. Geological Survey Open-File Report 02-0223, variously paginated.

U.S. Environmental Protection Agency, 1980, Onsite wastewater treatment and disposal systems design manual: Cincinnati, Ohio, U.S. Environmental Protection Agency report 625/1-80-012, $391 \mathrm{p}$.

U.S. Environmental Protection Agency, 1993, Guidance on evaluation, resolution, and documentation of analytical problems associated with compliance monitoring: Washington D.C., U.S. Environmental Protection Agency report 821-B-93-001, 56 p.
U.S. Environmental Protection Agency, 1997, Guidelines establishing test procedures for the analysis of pollutants (App. B, Part 136, Definition and procedures for the determination of the method detection limit): U.S. Code of Federal Regulations, Title 40, revised July 1, 1997, p. 265-267.

U.S. Environmental Protection Agency, 2001a, Method 1602: Male-specific $\left(\mathrm{F}^{+}\right)$and somatic coliphage in water by single agar layer (SAL) procedure: Washington D.C., U.S. Environmental Protection Agency report 821-R-01-029, 30 p.

U.S. Environmental Protection Agency, 2001b, Method 1601: Male-specific $\left(\mathrm{F}^{+}\right)$and somatic coliphage in water by twostep enrichment procedure: Washington, D.C., U.S. Environmental Protection Agency report 821-R-01-030, 32 p.

U.S. Geological Survey, 1999, National field manual for the collection of water-quality data: U.S. Geological Survey Techniques of Water-Resources Investigations, Book 9, Chaps. A1-A9, 2 v., variously paginated.

Yanko, W.A., Jackson, J.L., Williams, F.P., Walker, A.S., and M.S. Castillo, 1999, An unexpected temporal pattern of coliphage isolation in groundwaters sampled from wells at varied distances from reclaimed water recharge sites: Water Research, v. 33, p. 53-64.

Zaugg, S.D., Smith, S.G., Schroeder, M.P., Barber, L.B., and Burkhardt, M.R., 2002, Methods of analysis by the U.S. Geological Survey National Water Quality LaboratoryDetermination of wastewater compounds by polystyrenedivinylbenzene solid-phase extraction and capillary-column gas chromatography/mass spectrometry: U.S. Geological Survey Water-Resources Investigations Report 01-4186, 37 p. (Available on the internet at http://nwql.usgs.gov/Public/pubs/WRIR01-4186.html.)

Zwiener, C., Gremm, T.J., Frimmel, F.H., 2001, Pharmaceutical residues in the aquatic environment and their significance for drinking water production, in Kümmerer, Klaus, ed., Pharmaceuticals in the environment, Sources, fate, effects and risks: Berlin, Springer-Verlag, p. 81-89. 
Appendix A: Maps Showing Layouts of Onsite Wastewater Treatment System Drainfield Lines and Downgradient Monitoring Wells in the Innovative Systems Network 
This page left intentionally blank 


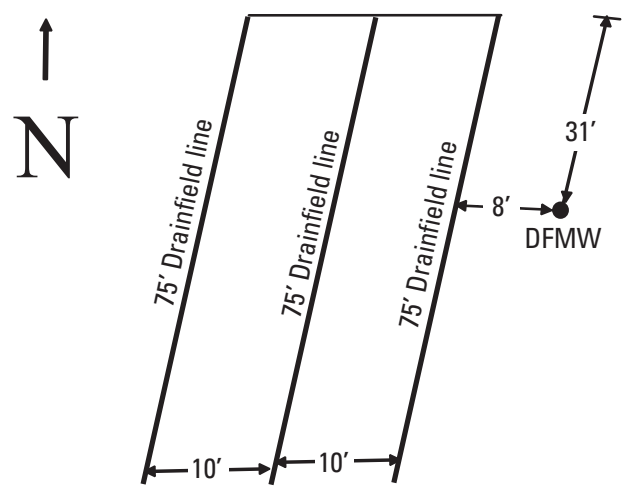

Drainfield Monitoring Well (DFMW) 434207121324601

Septic tank with gravity-feed drainfield lines

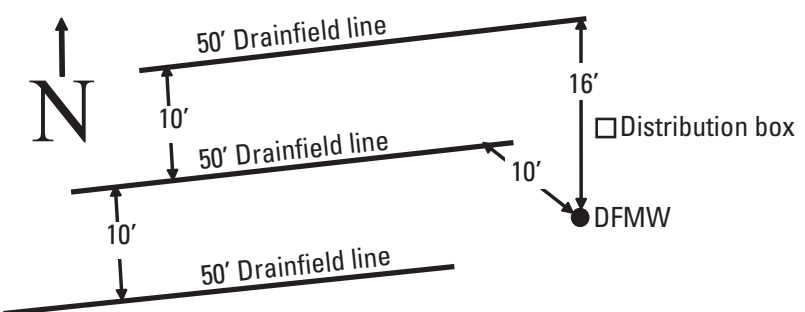

Drainfield Monitoring Well (DFMW) 434236121310501

Septic tank with gravity-feed drainfield lines

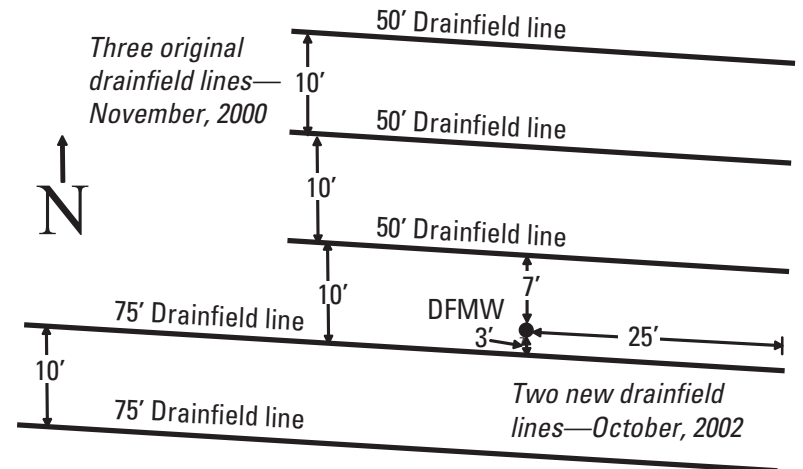

Drainfield Monitoring Well (DFMW) 434247121305501

Septic tank with pressurized drainfield lines

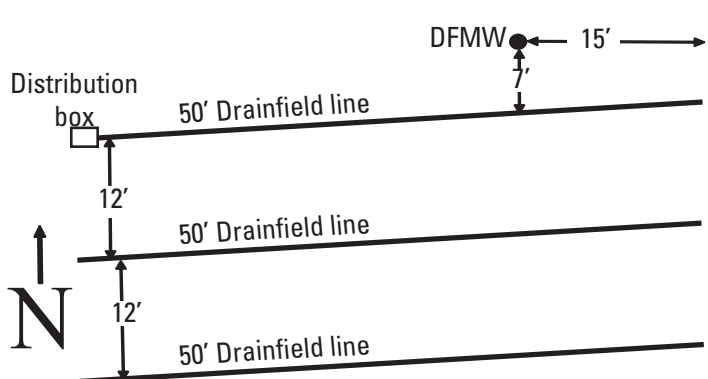

Drainfield Monitoring Well (DFMW) 434248121295901

Septic tank with pressurized drainfield lines

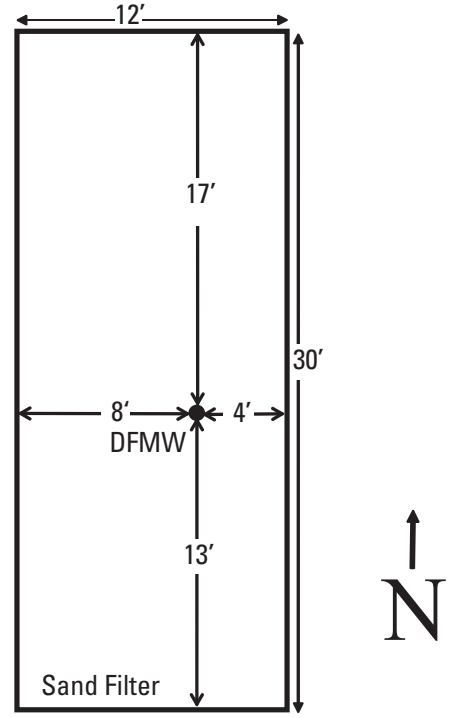

Drainfield Monitoring Well (DFMW) 434347121293901 Sand filter

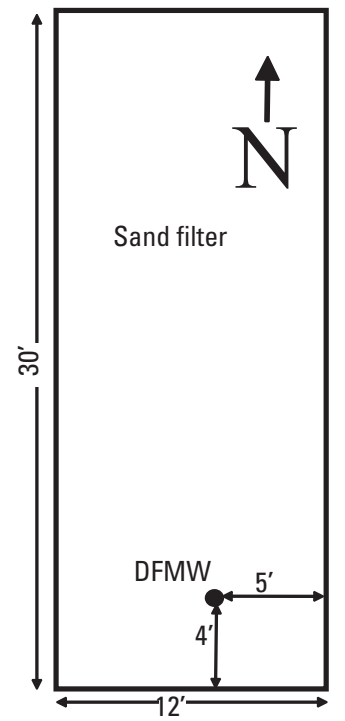

Drainfield Monitoring Well (DFMW) 434741121273101 Sand filter

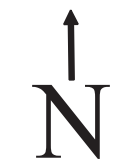

50 ' Drip irrigation lines

DFMW

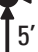

$25^{\prime}$

$50^{\prime}$ Drip irrigation lines

Drainfield Monitoring Well (DFMW) 434652121273001

AdvanTex (AX-20) onsite wastewater treatment system 


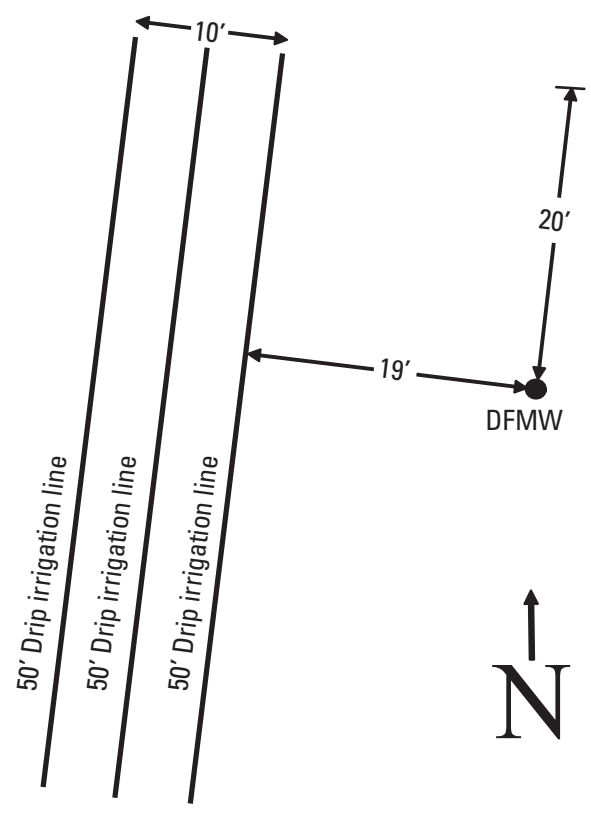

Drainfield Monitoring Well (DFMW) 434536121291201 AdvanTex (AX-20) onsite wastewater treatment system

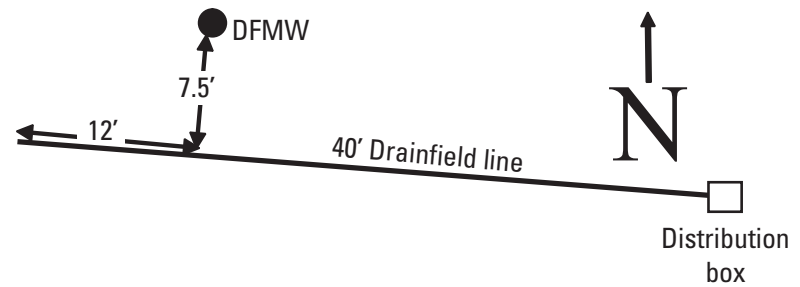

40' Drainfield line

Drainfield Monitoring Well (DFMW) 434011121314601 Amphidrome onsite wastewater treatment system

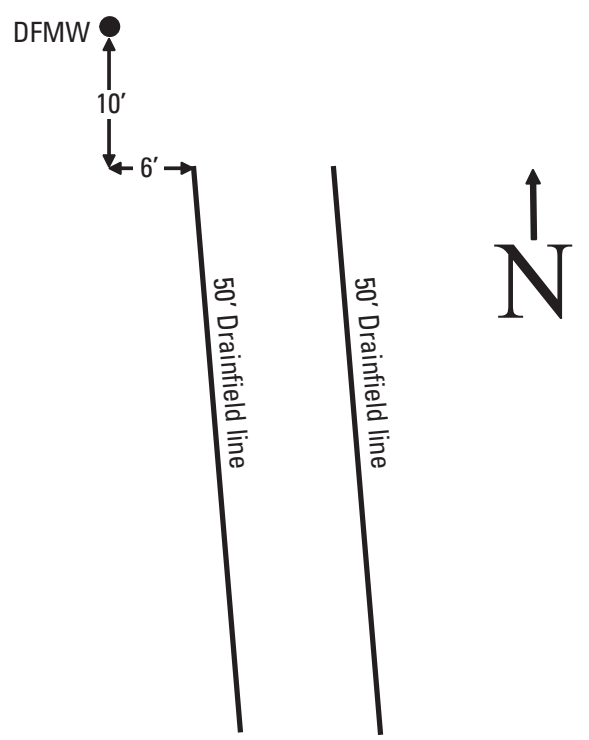

Drainfield Monitoring Well (DFMW) 434243121290101

Amphidrome onsite wastewater treatment system

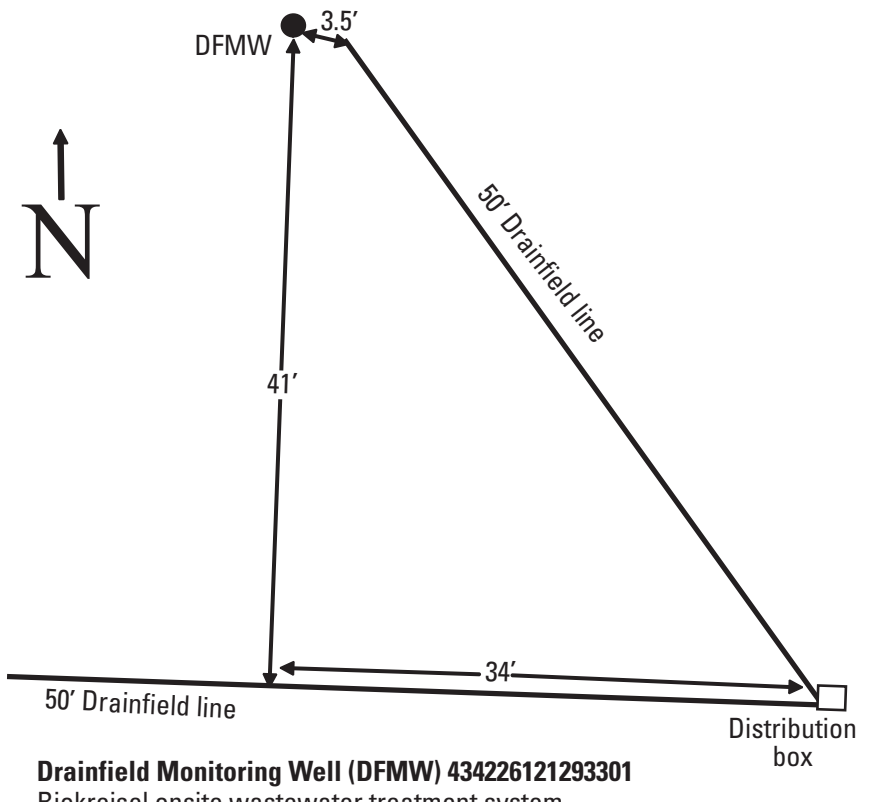

Biokreisel onsite wastewater treatment system

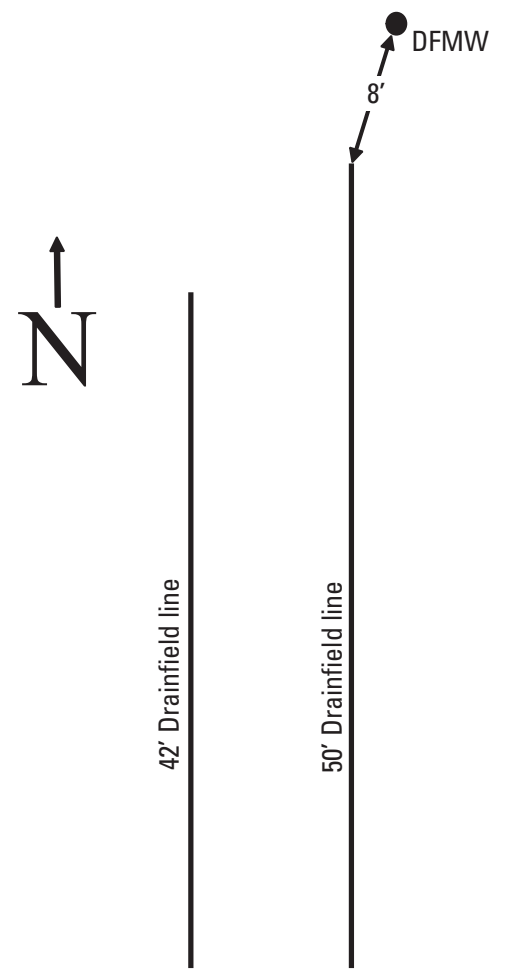

Drainfield Monitoring Well (DFMW) 434727121273701

Biokreisel onsite wastewater treatment system 

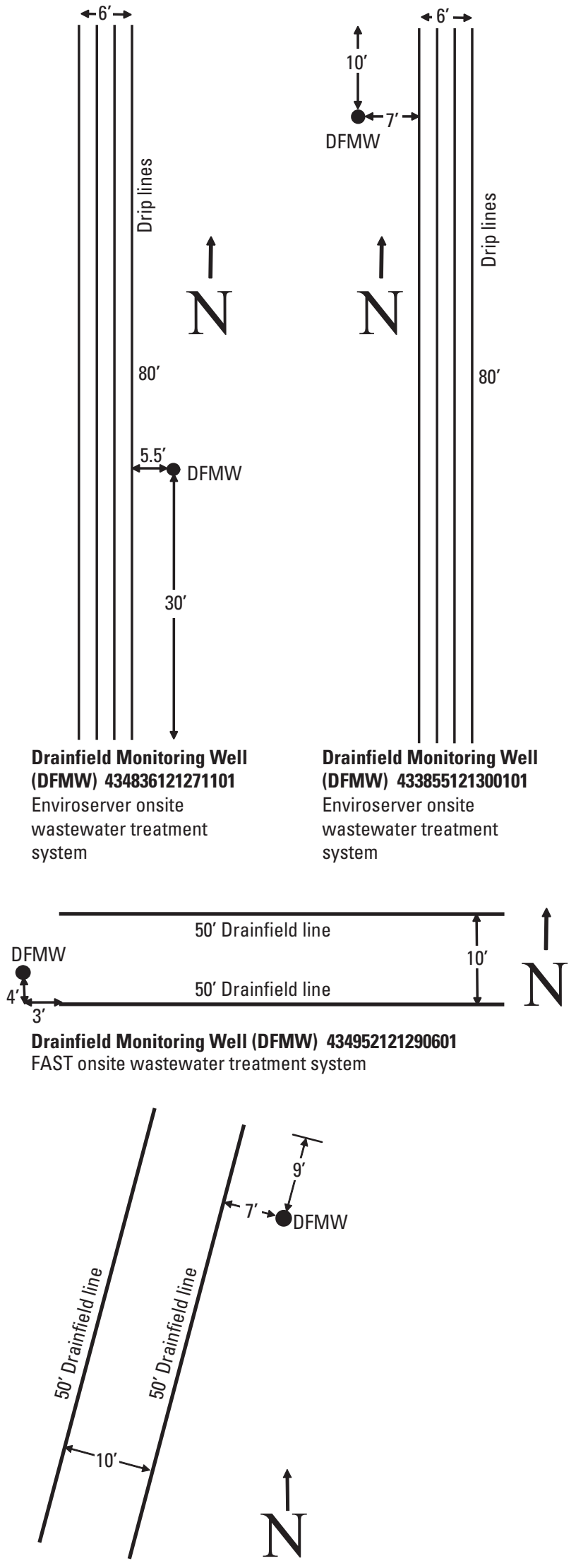

Drainfield Monitoring Well (DFMW) 434437121295301 FAST onsite wastewater treatment system

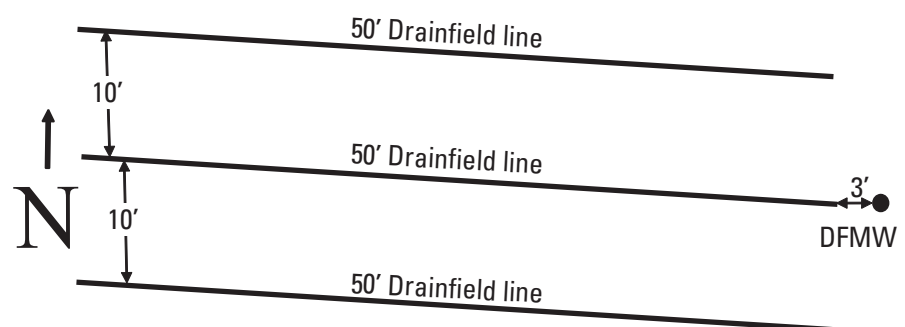

Drainfield Monitoring Well (DFMW) $\mathbf{4 3 5 0 1 6 1 2 1 2 8 4 7 0 1}$ NAYADIC onsite wastewater treatment system

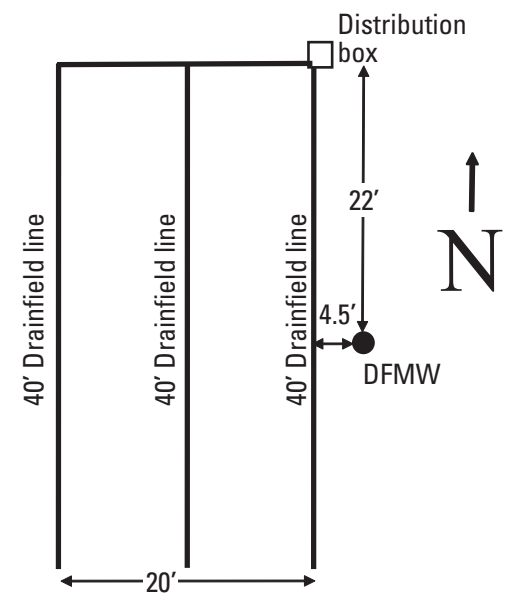

Drainfield Monitoring Well (DFMW) 434713121274301 NAYADIC onsite wastewater treatment system

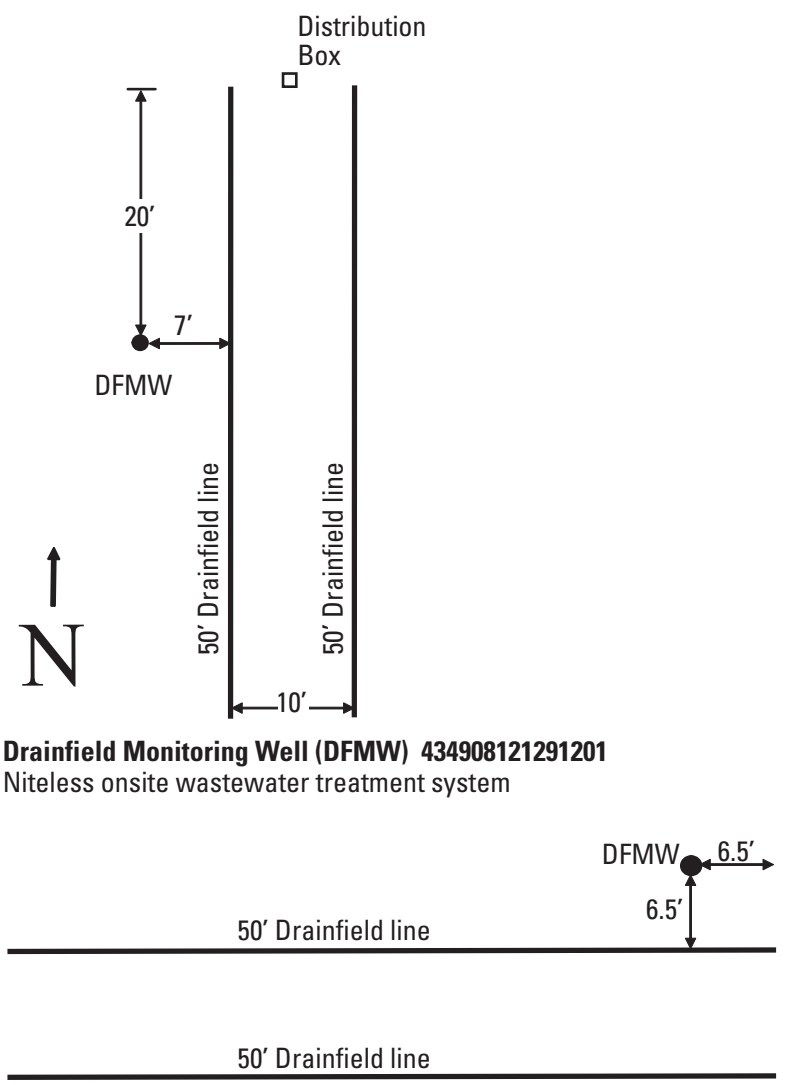

Drainfield Monitoring Well (DFMW) 434431121293501 Niteless onsite wastewater treatment system 


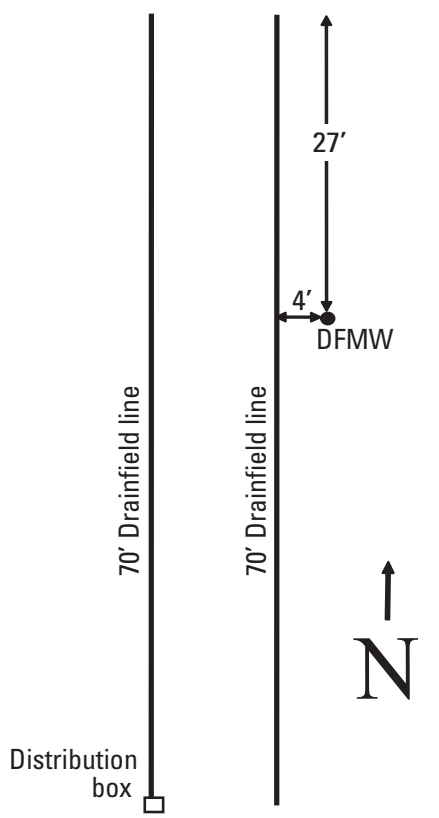

Drainfield Monitoring Well (DFMW) 434203121311201

NITREX onsite wastewater treatment system

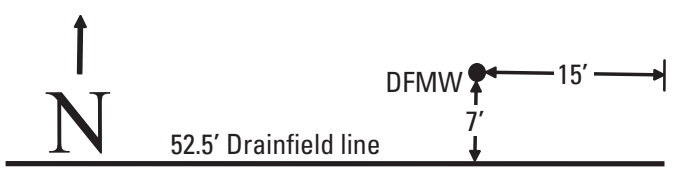

52.5' Drainfield line

Drainfield Monitoring Well (DFMW) 433825121340001 NITREX onsite wastewater treatment system

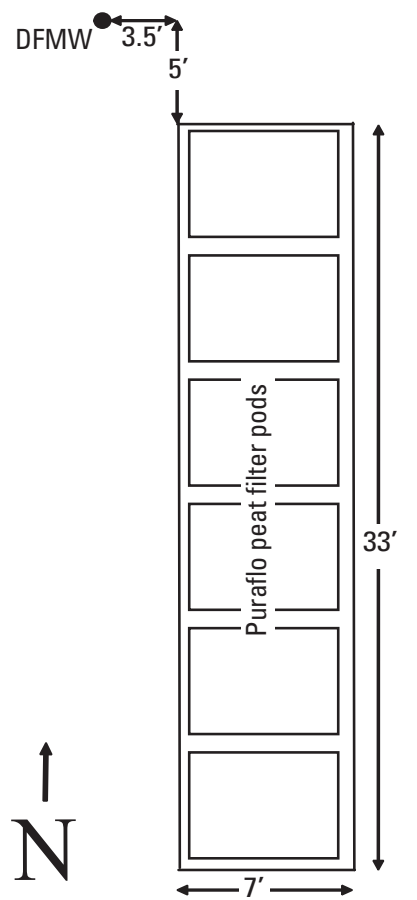

Drainfield Monitoring Well (DFMW) 434010121325601 Puraflow onsite wastewater treatment system

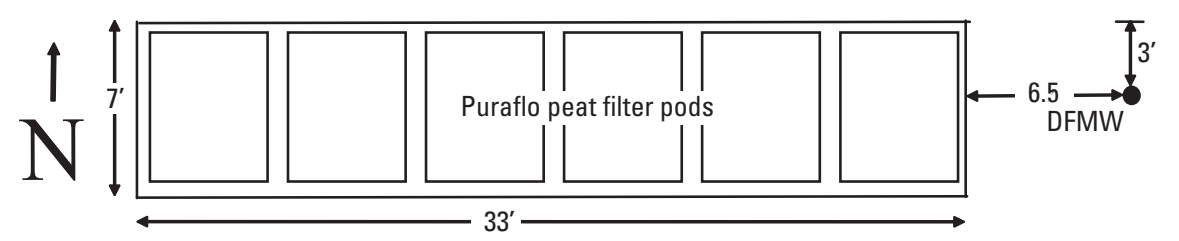

Drainfield Monitoring Well (DFMW) 434324121292601

Puraflow onsite wastewater treatment system

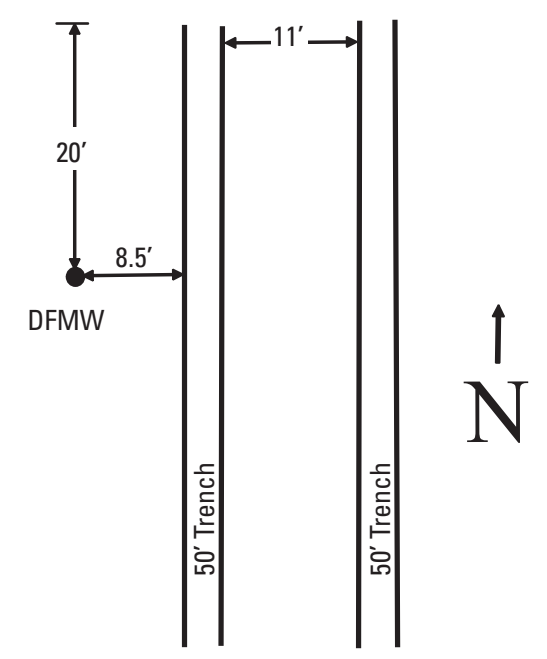

Drainfield Monitoring Well (DFMW) 434449121310201

Wert B onsite wastewater treatment system

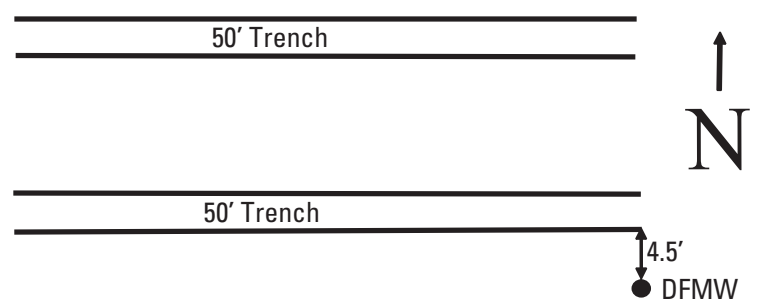

Drainfield Monitoring Well (DFMW) 434423121312901

Wert B onsite wastewater treatment system

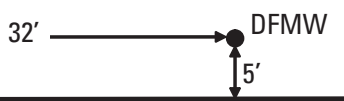

75' Drainfield line

75’ Drainfield line

Drainfield Monitoring Well (DFMW) 433950121322901

Dyno2 onsite wastewater treatment system

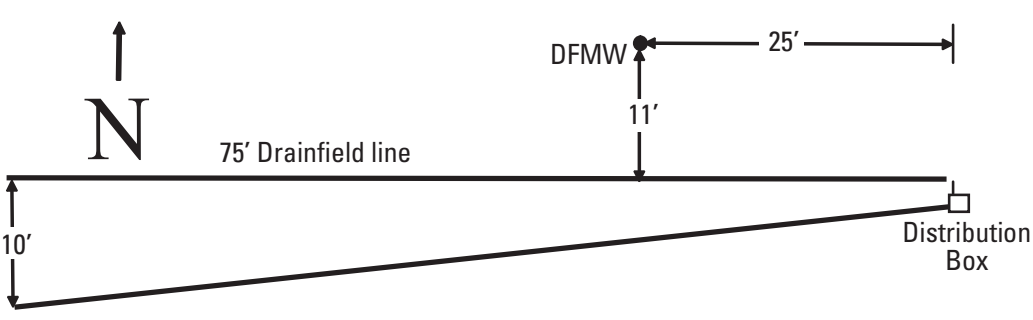

Drainfield Monitoring Well (DFMW) $\mathbf{4 3 4 1 3 1 1 2 1 3 1 4 3 0 1}$

Dyno2 onsite wastewater treatment system 


\section{Appendix B: Organic Wastewater Compound Quality Assurance}

Quality-control data were collected to assess organic wastewater compound sampling and analytical precision and bias. These data are discussed in this appendix.

\section{Organic Wastewater Compound Replicates}

Two sets of triplicate samples-one of septic tank effluent and one from a transect site-were collected and analyzed to measure analytical precision. These data are shown in table B1. Means and standard deviations were calculated in table B1 for analytes that were uncensored in all three analyses. Calculated standard deviations ranged from 0 to 37 percent of mean, with a median standard deviation of 12 percent of mean, and a mean standard deviation of 13 percent of mean. There were seven instances where censored and uncensored values were reported for the same organic wastewater compound among the triplicate samples. In five of these seven instances, one or more results was/were censored at a value greater than or equal to one or more uncensored results (e.g., a value of $<0.5 \mu \mathrm{g} / \mathrm{L}$ with values of E0.2 $\mu \mathrm{g} / \mathrm{L}$ and E0.3 $\mu \mathrm{g} / \mathrm{L}$ ). In two instances, results censored at 5 or $6 \mu \mathrm{g} / \mathrm{L}$ were reported along with results $>5$ or $6 \mu \mathrm{g} / \mathrm{L}$ (diethoxynonylphenol: $<5$, E9, and E14 $\mu \mathrm{g} / \mathrm{L}$, and 4-nonylphenol: <6, E10, and E10 $\mu \mathrm{g} / \mathrm{L}$ ), not unreasonable uncertainty given that the medians for these compounds were within a factor of two of the LRLs.

\section{Organic Wastewater Compound Matrix Spike}

One sample of septic tank effluent was spiked at the NWQL with known concentrations of organic wastewater compounds. The resultant information provides a measure of analytical bias, specifically, of analyte recovery. These data, including calculated analyte recoveries, are shown in table B2.

Recovery is calculated as follows:

recovered spike concentration $=($ concentration in spiked sample) - (concentration in environmental sample) and

$$
\begin{gathered}
\text { recovery }(\text { percent })=100 \times(\text { recovered spike } \\
\text { concentration }) \div(\text { expected concentration from spike })
\end{gathered}
$$

Most analytes were spiked to deliver a concentration of several micrograms per liter. Spiking at such concentrations provides recovery data for concentrations representative of those often seen in environmental samples. In several instances, the analyte mass in the environmental sample prior to spiking was much greater than the analyte mass added in the laboratory. This often arises when spiking a sample that contains high concentrations of organic wastewater compounds, and septic tank effluent certainly represents such a case. The result of adding a small mass of analyte to an environmental sample containing a large mass of that analyte is that slight variability in analysis of the unspiked and spiked samples might be attributed to the addition of analyte through the spiking process, resulting in large apparent positive (or even negative) recoveries. This procedure artifact was illustrated with the septic tank effluent sample in table B2, where p-cresol was reported at $730 \mu \mathrm{g} / \mathrm{L}$ in the environmental sample and $790 \mu \mathrm{g} / \mathrm{L}$ in the spiked sample (the spiked sample contained $5.8 \mu \mathrm{g} / \mathrm{L}$ from the spiking process). The difference between $730 \mu \mathrm{g} / \mathrm{L}$ and $790 \mu \mathrm{g} / \mathrm{L}$ could be entirely attributed to analytical variability (see discussion of replicates); the addition of p-cresol from spiking was dwarfed by the analytical imprecision for p-cresol in the environmental sample. This limitation should be considered when evaluating spike recoveries.

Analyte recovery in table B2 is censored with a " $\leq$ " when the analyte concentration in the unspiked sample was censored (reported as a non-detect), or qualified with an " $\mathrm{M}$ " (presence verified, not quantified). For example, 1,4-dichlorobenzene was reported at $<5 \mu \mathrm{g} / \mathrm{L}$ in the unspiked sample, and $\mathrm{E} 3.6 \mu \mathrm{g} / \mathrm{L}$ in the spiked sample. If the dichlorobenzene concentration that was reported as $<5 \mu \mathrm{g} / \mathrm{L}$ had been present at $0 \mu \mathrm{g} / \mathrm{L}$, the recovery would have been 62 percent. If the dichlorobenzene concentration that was reported as $<5 \mu \mathrm{g} / \mathrm{L}$ had been present at $>0 \mu \mathrm{g} / \mathrm{L}$, the recovery would have been $<62$ percent. Recovery of 1,4-dichlorobenzene thus is reported to be $\leq 62$ percent. For the purpose of summarizing results in this paragraph, though, the assumption is made that nondetects and " $\mathrm{M}$ " coded concentrations were present at $0 \mu \mathrm{g} / \mathrm{L}$. Under this assumption, recoveries ranged from 22 percent to 1,000 percent of expected concentration. (The 1,000 percent recovery was for p-cresol). Of the 63 compounds, 79 percent had recoveries between 60 and 140 percent. The median recovery (all 63 compounds) was 79 percent, and the mean recovery 110 percent. Note that if analytes were present in the unspiked sample but were reported as nondetects or " $\mathrm{M}$ " coded concentrations, the effect would be to impart a high bias in the calculated recoveries.

\section{Organic Wastewater Compound Surrogate Recoveries}

Surrogate compounds were added to all organic wastewater compound samples to evaluate method performance. Surrogate compounds are compounds with chemical properties similar to those of some of the compounds being analyzed for in environmental samples. Surrogate compounds are not expected to be naturally present in environmental samples. Method performance for surrogate compounds is expected to reflect method performance for environmental organic wastewater compounds that are chemically similar to surrogate compounds. Surrogate compound recovery is reported along with organic wastewater compound data for both environmental and quality-control samples in their respective data tables. A summary of surrogate compound recovery for La Pine 
environmental samples is shown in table B3. For comparative purposes, table B3 also lists the mean surrogate recoveries for all NWQL organic wastewater compound samples analyzed during calendar year 2003 (1,448 samples). Organic wastewater compound concentrations are not adjusted on the basis of surrogate recoveries.

Recovery of bisphenol A-d3 (mean recovery 69.1 percent) and decafluorobiphenyl (mean recovery 75.7 percent) was lower than for fluoranthene-d10 (mean recovery 93.6 percent) and caffeine-13C (mean recovery 99.7 percent). However, mean recovery for each of the four surrogate compounds in La Pine environmental samples was similar to the mean recovery for these surrogate compounds in the set of 1448 NWQL organic wastewater compound samples (table B3).

\section{Organic Wastewater Compound Field Equipment Blanks}

Two field equipment blanks were collected by passing organic-blank water (provided by the USGS NWQL, after testing to ensure purity) through sample collection equipment immediately prior to collecting environmental samples. Field equipment blanks were filtered in the same manner as were environmental samples. Analysis of field equipment blanks provides a measure of sampling, processing, and analytical bias, specifically, of contamination from sample collection, filtering and analysis. Field equipment blank results are shown in table B4.
Three analytes were detected in field equipment blanks: 1,4-dichlorobenzene ("M", presence verified, not quantified), p-cresol ("M"), and phenol (E0.4 $\mu \mathrm{g} / \mathrm{L})$. The presence of two low-level, "M" coded detections in field equipment blanks is not surprising, given the fact that many organic wastewater compounds are ubiquitous chemicals. More extensive quality assurance is needed to potentially identify the exact source. However, the presence of two " $M$ " coded results in field equipment blanks suggests that all "M" coded environmental data should be treated with caution. One means of interpreting the presence of " $M$ " coded environmental data would be to consider them to be nondetects at the LRL; this was done for analysis purposes in this document.

The presence of phenol in one field equipment blank at a concentration of E0.4 $\mu \mathrm{g} / \mathrm{L}$ casts some doubt on the reliability of the LRL of $0.5 \mu \mathrm{g} / \mathrm{L}$ for phenol. The reported presence of phenol in environmental samples may be better represented at a censoring level of 10 times the contamination level of 0.4 $\mu \mathrm{g} / \mathrm{L}$, for a project censoring level of $4 \mu \mathrm{g} / \mathrm{L}$.

These two field equipment blanks demonstrated that collection, filtering and analysis of samples for organic wastewater compounds, except for phenol, can be accomplished with contamination levels below those quantifiable by the analytical techniques used. For phenol, a project censoring level of $4 \mu \mathrm{g} / \mathrm{L}$ may provide a conservative approach for evaluating detection frequency. 
Table B1. Results of replicate analyses for organic wastewater compound samples, La Pine, Oregon, 2003.

[Date as year, month, day (YYYYMMDD); Time in hours and minutes, military; organic wastewater compounds in micrograms per liter; surrogate recoveries in percent; parameter codes for organic wastewater compound names: Pxxxx; "E", estimated;" >", less than; "M", presence verified, not quantified; —, no data]

\begin{tabular}{|c|c|c|c|c|c|c|c|c|c|c|c|c|}
\hline Location of sample & Sample type & $\begin{array}{l}\text { Station number or } \\
\text { summary statistic }\end{array}$ & Date & Time & 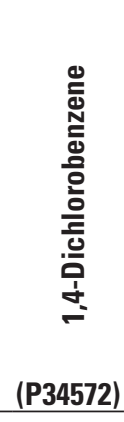 & (P62054) & 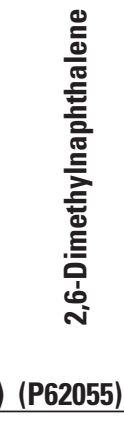 & 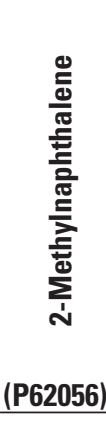 & 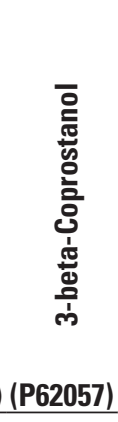 & ) (P62058) & 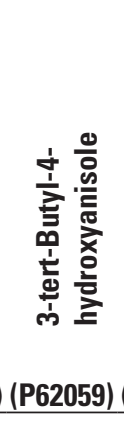 & 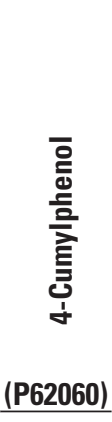 \\
\hline \multirow[t]{5}{*}{ Transect } & \multirow[t]{5}{*}{ Ground water } & 434212121294208 & 20030501 & 1500 & $<0.5$ & $<0.5$ & $<0.5$ & $<0.5$ & $<2$ & $<1$ & $<5$ & $<1$ \\
\hline & & 434212121294208 & 20030501 & 1501 & $<0.5$ & $<0.5$ & $<0.5$ & $<0.5$ & M & $<1$ & $<5$ & $<1$ \\
\hline & & 434212121294208 & 20030501 & 1502 & $<0.5$ & $<0.5$ & $<0.5$ & $<0.5$ & M & $<1$ & $<5$ & $<1$ \\
\hline & & Mean & - & - & - & - & - & - & - & - & - & - \\
\hline & & Standard deviation & - & - & - & - & - & - & - & - & - & - \\
\hline \multirow[t]{5}{*}{ NAYADIC onsite wastewater treatment system } & \multirow[t]{5}{*}{ Septic tank effluent } & 435016121284702 & 20030407 & 1200 & $<5$ & E0.2 & $<5$ & E0.2 & E11 & 52 & $<5$ & $<5$ \\
\hline & & 435016121284702 & 20030407 & 1201 & $<0.5$ & E0.2 & $<0.5$ & E0.2 & 14 & 39 & $<5$ & $<1$ \\
\hline & & 435016121284702 & 20030407 & 1202 & M & E0.2 & $<5$ & E0.2 & 11 & 42 & $<5$ & $<5$ \\
\hline & & Mean & - & - & - & E0.2 & & E0.2 & E12 & 44 & - & - \\
\hline & & Standard deviation & - & - & - & 0.0 & - & 0.0 & 2 & 7 & - & - \\
\hline
\end{tabular}


Table B1. Results of replicate analyses for organic wastewater compound samples, La Pine, Oregon, 2003.—Continued

[Date as year, month, day (YYYYMMDD); Time in hours and minutes, military; organic wastewater compounds in micrograms per liter; surrogate recoveries in percent;

parameter codes for organic wastewater compound names: Pxxxx; "E", estimated;"<", less than; "M", presence verified, not quantified; -, no data]

\begin{tabular}{|c|c|c|c|c|c|c|c|c|c|c|c|c|}
\hline Location of sample & Sample type & $\begin{array}{l}\text { Station number or } \\
\text { summary statistic }\end{array}$ & Date & Time & 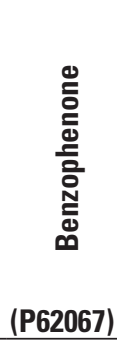 & 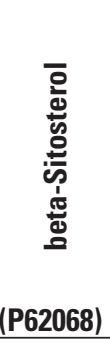 & 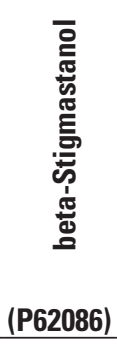 & 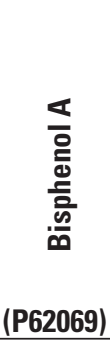 & 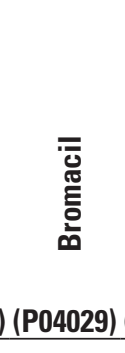 & 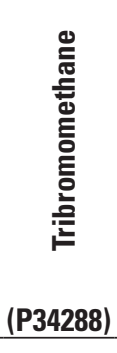 & 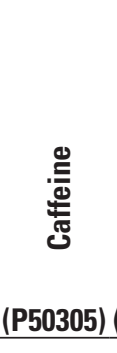 & $\begin{array}{l}\text { 흐 } \\
\text { 言 } \\
\text { 产 }\end{array}$ \\
\hline \multirow[t]{5}{*}{ Transect } & \multirow[t]{5}{*}{ Ground water } & 434212121294208 & 20030501 & 1500 & $<0.5$ & $<2$ & $<2$ & $<1$ & $<0.5$ & $<0.5$ & $<0.5$ & $<0.5$ \\
\hline & & 434212121294208 & 20030501 & 1501 & $<0.5$ & E1 & E2 & $<1$ & $<0.5$ & $<0.5$ & $<0.5$ & $<0.5$ \\
\hline & & 434212121294208 & 20030501 & 1502 & $<0.5$ & E2 & E2 & $<1$ & $<0.5$ & $<0.5$ & $<0.5$ & $<0.5$ \\
\hline & & Mean & - & - & - & - & - & - & - & - & - & - \\
\hline & & Standard deviation & - & - & - & - & - & - & - & - & - & - \\
\hline \multirow[t]{5}{*}{ NAYADIC onsite wastewater treatment system } & \multirow[t]{5}{*}{ Septic tank effluent } & 435016121284702 & 20030407 & 1200 & 2.2 & E12 & E8 & M & $<5$ & $<5$ & 18 & 0.8 \\
\hline & & 435016121284702 & 20030407 & 1201 & 2.4 & 14 & 8 & M & $<0.5$ & $<0.5$ & 14 & 0.7 \\
\hline & & 435016121284702 & 20030407 & 1202 & 2.3 & 10 & 7 & M & $<5$ & $<5$ & 20 & 0.8 \\
\hline & & Mean & - & - & 2.3 & E12 & E8 & - & - & - & 17 & 0.8 \\
\hline & & Standard deviation & $=$ & - & 0.1 & 2 & & $=$ & - & - & 3 & 0.1 \\
\hline
\end{tabular}


[Date as year, month, day (YYYYMMDD); Time in hours and minutes, military; organic wastewater compounds in micrograms per liter; surrogate recoveries in percent;

parameter codes for organic wastewater compound names: Pxxxx; "E", estimated;"<", less than; "M", presence verified, not quantified; -, no data]

\begin{tabular}{|c|c|c|c|c|c|c|c|c|c|c|c|c|}
\hline Location of sample & Sample type & $\begin{array}{l}\text { Station number or } \\
\text { summary statistic }\end{array}$ & Date & Time & 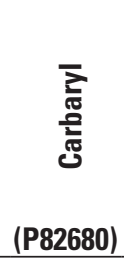 & 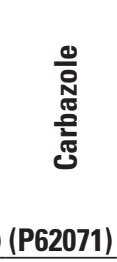 & 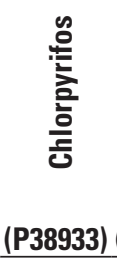 & 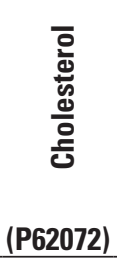 & 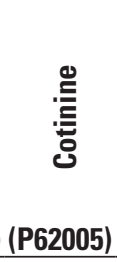 & 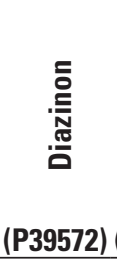 & 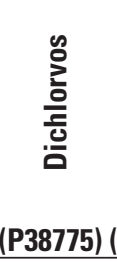 & 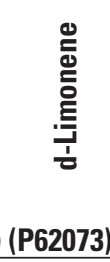 \\
\hline \multirow[t]{4}{*}{ Transect } & \multirow[t]{4}{*}{ Ground water } & 434212121294208 & 20030501 & 1500 & $<1$ & $<0.5$ & $<0.5$ & $<2$ & $<1$ & $<0.5$ & $<1$ & $<0.5$ \\
\hline & & 434212121294208 & 20030501 & 1501 & $<1$ & $<0.5$ & $<0.5$ & E1 & $<1$ & $<0.5$ & $<1$ & $<0.5$ \\
\hline & & 434212121294208 & 20030501 & 1502 & $<1$ & $<0.5$ & $<0.5$ & E2 & $<1$ & $<0.5$ & $<1$ & $<0.5$ \\
\hline & & Standard deviation & - & - & - & - & - & - & - & - & - & - \\
\hline \multirow[t]{4}{*}{ NAYADIC onsite wastewater treatment system } & \multirow[t]{4}{*}{ Septic tank effluent } & 435016121284702 & 20030407 & 1200 & $<5$ & $<5$ & $<5$ & E32 & $<5$ & $<5$ & $<5$ & E8.9 \\
\hline & & 435016121284702 & 20030407 & 1201 & $<1$ & $<0.5$ & $<0.5$ & 32 & $<1$ & $<0.5$ & $<1$ & E17 \\
\hline & & 435016121284702 & 20030407 & 1202 & $<5$ & $<5$ & $<5$ & 32 & $<5$ & $<5$ & $<5$ & E10 \\
\hline & & Mean & - & - & - & - & - & E32 & - & - & - & E12 \\
\hline
\end{tabular}


Table B1. Results of replicate analyses for organic wastewater compound samples, La Pine, Oregon, 2003.—Continued

[Date as year, month, day (YYYYMMDD); Time in hours and minutes, military; organic wastewater compounds in micrograms per liter; surrogate recoveries in percent;

parameter codes for organic wastewater compound names: Pxxxx; "E", estimated;"<", less than; "M", presence verified, not quantified; -, no data]

\begin{tabular}{|c|c|c|c|c|c|c|c|c|c|c|c|c|}
\hline Location of sample & Sample type & $\begin{array}{l}\text { Station number or } \\
\text { summary statistic }\end{array}$ & Date & Time & 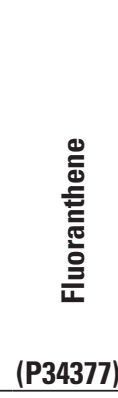 & 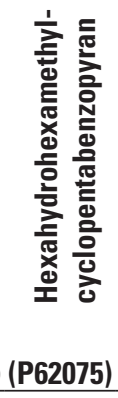 & 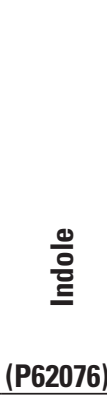 & 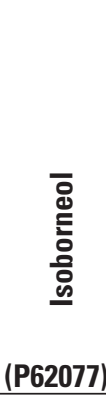 & $\begin{array}{c}\text { 음 } \\
\text { 음 } \\
\text { 응 } \\
\text { ) (P34409) }\end{array}$ & 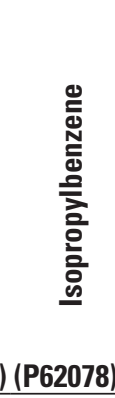 & 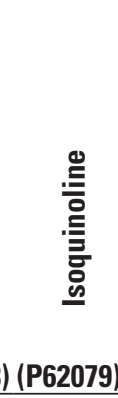 & 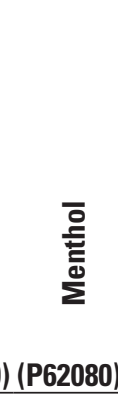 \\
\hline \multirow[t]{5}{*}{ Transect } & \multirow[t]{5}{*}{ Ground water } & 434212121294208 & 20030501 & 1500 & $<0.5$ & $<0.5$ & $<0.5$ & $<0.5$ & $<0.5$ & $<0.5$ & $<0.5$ & $<0.5$ \\
\hline & & 434212121294208 & 20030501 & 1501 & $<0.5$ & $<0.5$ & $<0.5$ & $<0.5$ & $<0.5$ & $<0.5$ & $<0.5$ & $<0.5$ \\
\hline & & 434212121294208 & 20030501 & 1502 & $<0.5$ & $<0.5$ & $<0.5$ & $<0.5$ & $<0.5$ & $<0.5$ & $<0.5$ & $<0.5$ \\
\hline & & Mean & - & - & - & - & - & - & - & - & - & - \\
\hline & & Standard deviation & - & - & - & - & - & - & - & - & - & - \\
\hline \multirow[t]{4}{*}{ NAYADIC onsite wastewater treatment system } & \multirow[t]{4}{*}{ Septic tank effluent } & 435016121284702 & 20030407 & 1200 & $<5$ & 1.2 & 34 & $<5$ & $<5$ & $<5$ & $<5$ & 62 \\
\hline & & 435016121284702 & 20030407 & 1201 & $<0.5$ & 1.3 & 21 & $<0.5$ & $<0.5$ & $<0.5$ & $<0.5$ & 62 \\
\hline & & 435016121284702 & 20030407 & 1202 & $<5$ & 1.2 & 30 & $<5$ & $<5$ & $<5$ & $<5$ & 50 \\
\hline & & $\begin{array}{l}\text { Mean } \\
\text { Standard deviation }\end{array}$ & - & - & - & $\begin{array}{l}1.2 \\
0.1\end{array}$ & $\begin{array}{r}28 \\
7\end{array}$ & - & - & - & - & $\begin{array}{r}58 \\
7\end{array}$ \\
\hline
\end{tabular}


[Date as year, month, day (YYYYMMDD); Time in hours and minutes, military; organic wastewater compounds in micrograms per liter; surrogate recoveries in percent;

parameter codes for organic wastewater compound names: Pxxxx; "E", estimated;"<", less than; "M", presence verified, not quantified; -, no data]

\begin{tabular}{|c|c|c|c|c|c|c|c|c|c|c|c|c|}
\hline Location of sample & Sample type & $\begin{array}{l}\text { Station number or } \\
\text { summary statistic }\end{array}$ & Date & Time & 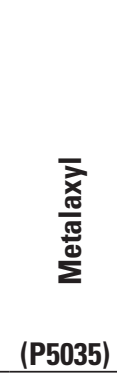 & 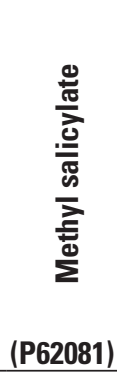 & 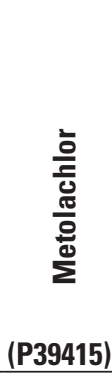 & 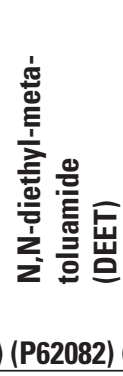 & 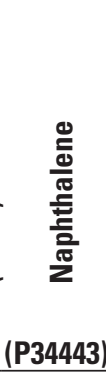 & 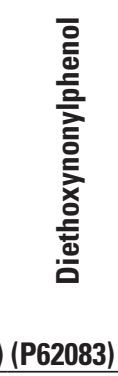 & 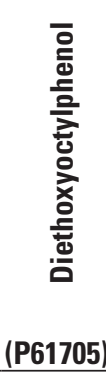 & 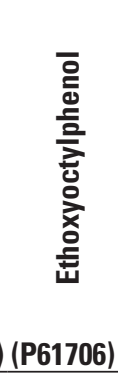 \\
\hline \multirow[t]{5}{*}{ Transect } & \multirow[t]{5}{*}{ Ground water } & 434212121294208 & 20030501 & 1500 & $<0.5$ & $<0.5$ & $<0.5$ & $<0.5$ & $<0.5$ & $<5$ & $<1$ & $<1$ \\
\hline & & 434212121294208 & 20030501 & 1501 & $<0.5$ & $<0.5$ & $<0.5$ & $<0.5$ & $<0.5$ & M & $<1$ & $<1$ \\
\hline & & 434212121294208 & 20030501 & 1502 & $<0.5$ & $<0.5$ & $<0.5$ & $<0.5$ & $<0.5$ & M & $<1$ & $<1$ \\
\hline & & Mean & - & - & - & - & - & - & - & - & - & - \\
\hline & & Standard deviation & - & - & - & - & - & - & - & - & - & - \\
\hline \multirow[t]{5}{*}{ NAYADIC onsite wastewater treatment system } & \multirow[t]{5}{*}{ Septic tank effluent } & 435016121284702 & 20030407 & 1200 & $<5$ & $<5$ & $<5$ & 0.6 & E0.2 & E9 & $<5$ & $<5$ \\
\hline & & 435016121284702 & 20030407 & 1201 & $<0.5$ & $<0.5$ & $<0.5$ & 0.7 & E0.2 & E14 & $<1$ & $<1$ \\
\hline & & 435016121284702 & 20030407 & 1202 & $<5$ & $<5$ & $<5$ & 0.6 & E0.2 & $<5$ & $<5$ & $<5$ \\
\hline & & Mean & - & - & - & - & - & 0.6 & E0.2 & - & - & - \\
\hline & & Standard deviation & - & - & - & - & - & 0.1 & 0.0 & - & - & - \\
\hline
\end{tabular}


Table B1. Results of replicate analyses for organic wastewater compound samples, La Pine, Oregon, 2003.—Continued

[Date as year, month, day (YYYYMMDD); Time in hours and minutes, military; organic wastewater compounds in micrograms per liter; surrogate recoveries in percent;

parameter codes for organic wastewater compound names: Pxxxx; "E", estimated;"<", less than; "M", presence verified, not quantified; -, no data]

\begin{tabular}{|c|c|c|c|c|c|c|c|c|c|c|c|c|}
\hline Location of sample & Sample type & $\begin{array}{l}\text { Station number or } \\
\text { summary statistic }\end{array}$ & Date & Time & 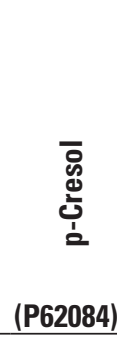 & 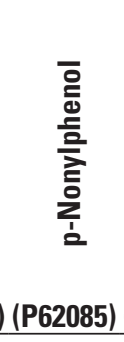 & 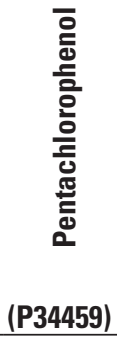 & 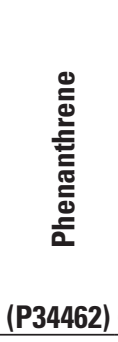 & $\begin{array}{c}\overline{\bar{O}} \\
\overline{\frac{D}{0}} \\
\text { (P34466) }\end{array}$ & $\begin{array}{r}\text { 흥 } \\
\text { 홍 } \\
\text { (P04037) }\end{array}$ & 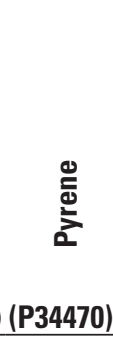 & 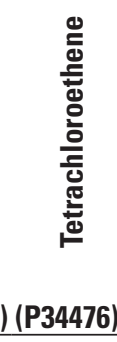 \\
\hline \multirow[t]{4}{*}{ Transect } & \multirow[t]{4}{*}{ Ground water } & 434212121294208 & 20030501 & 1500 & $<1$ & $<6$ & $<2$ & $<0.5$ & $<0.5$ & $<0.5$ & $<0.5$ & E0.1 \\
\hline & & 434212121294208 & 20030501 & 1502 & $<1$ & $<6$ & $<2$ & $<0.5$ & E0.3 & $<0.5$ & $<0.5$ & E0.1 \\
\hline & & Mean & - & - & - & - & - & - & - & - & - & E0.1 \\
\hline & & Standard deviation & - & - & - & - & - & - & - & - & - & 0.0 \\
\hline \multirow[t]{2}{*}{ NAYADIC onsite wastewater treatment system } & \multirow[t]{2}{*}{ Septic tank effluent } & 435016121284702 & 20030407 & 1200 & 730 & E10 & $<5$ & $<5$ & 240 & $<5$ & $<5$ & $<5$ \\
\hline & & 435016121284702 & 20030407 & 1201 & 370 & E10 & $<2$ & $<0.5$ & 130 & $<0.5$ & $<0.5$ & $<0.5$ \\
\hline
\end{tabular}


[Date as year, month, day (YYYYMMDD); Time in hours and minutes, military; organic wastewater compounds in micrograms per liter; surrogate recoveries in percent;

parameter codes for organic wastewater compound names: Pxxxx; "E", estimated;"<", less than; "M", presence verified, not quantified; -, no data]

\begin{tabular}{|c|c|c|c|c|c|c|c|c|c|c|c|c|}
\hline Location of sample & Sample type & $\begin{array}{l}\text { Station number or } \\
\text { summary statistic }\end{array}$ & Date & Time & 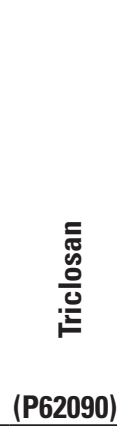 & 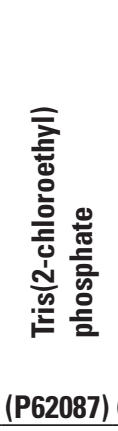 & 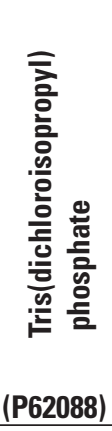 & 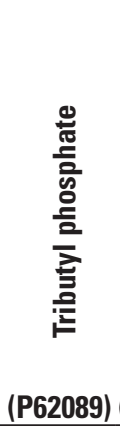 & 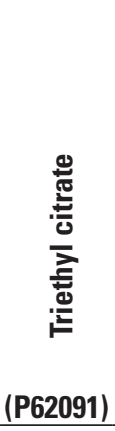 & 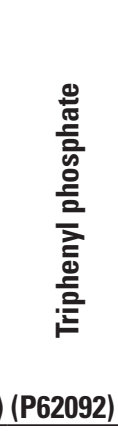 & 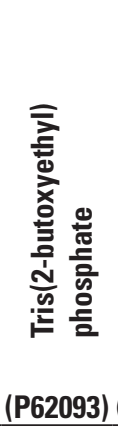 & 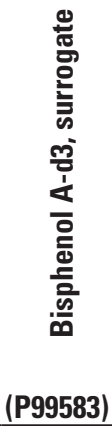 \\
\hline \multirow{5}{*}{ Transect } & \multirow[t]{5}{*}{ Ground water } & 434212121294208 & 20030501 & 1500 & $<1$ & $<0.5$ & $<0.5$ & $<0.5$ & $<0.5$ & $<0.5$ & $<0.5$ & 100 \\
\hline & & 434212121294208 & 20030501 & 1501 & $<1$ & $<0.5$ & $<0.5$ & $<0.5$ & $<0.5$ & $<0.5$ & $<0.5$ & 104 \\
\hline & & 434212121294208 & 20030501 & 1502 & $<1$ & $<0.5$ & $<0.5$ & $<0.5$ & $<0.5$ & $<0.5$ & $<0.5$ & 100 \\
\hline & & Mean & - & - & - & - & - & - & - & - & - & - \\
\hline & & Standard deviation & - & - & - & - & - & - & - & - & - & - \\
\hline \multirow[t]{5}{*}{ NAYADIC onsite wastewater treatment system } & \multirow[t]{5}{*}{ Septic tank effluent } & 435016121284702 & 20030407 & 1200 & E2 & E0.4 & E0.4 & $<5$ & 0.6 & 0.8 & $\mathrm{E} 2.2$ & 78.6 \\
\hline & & 435016121284702 & 20030407 & 1201 & 2 & E0.4 & E0.3 & $<0.5$ & 0.6 & 0.8 & 2.4 & 80.8 \\
\hline & & 435016121284702 & 20030407 & 1202 & E2 & E0.4 & E0.3 & $<5$ & 0.7 & 0.8 & $\mathrm{E} 2.2$ & 78.4 \\
\hline & & Mean & - & - & E2 & E0.4 & E0.3 & - & 0.6 & 0.8 & E2.3 & - \\
\hline & & Standard deviation & - & - & 0 & 0.0 & 0.1 & - & 0.1 & 0.0 & 0.1 & - \\
\hline
\end{tabular}


Table B1. Results of replicate analyses for organic wastewater compound samples, La Pine, Oregon, 2003.—Continued

[Date as year, month, day (YYYYMMDD); Time in hours and minutes, military; organic wastewater compounds in micrograms per liter; surrogate recoveries in percent; parameter codes for organic wastewater compound names: Pxxxx; "E", estimated;"<", less than; "M", presence verified, not quantified; - , no data]

\begin{tabular}{|c|c|c|c|c|c|c|c|}
\hline Location of sample & Sample type & $\begin{array}{l}\text { Station number or } \\
\text { summary statistic }\end{array}$ & Date & Time & 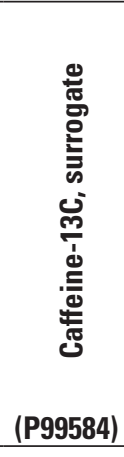 & 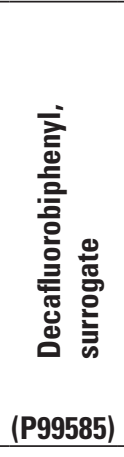 & 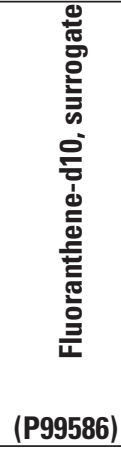 \\
\hline \multirow[t]{5}{*}{ Transect } & Ground water & 434212121294208 & 20030501 & 1500 & 122 & 87.0 & 104 \\
\hline & & 434212121294208 & 20030501 & 1501 & 117 & 104 & 126 \\
\hline & & 434212121294208 & 20030501 & 1502 & 118 & 105 & 123 \\
\hline & & Mean & - & - & - & - & - \\
\hline & & Standard deviation & - & - & - & - & - \\
\hline \multirow[t]{5}{*}{ NAYADIC onsite wastewater treatment system } & Septic tank effluent & 435016121284702 & 20030407 & 1200 & 133 & 59.3 & 81.1 \\
\hline & & 435016121284702 & 20030407 & 1201 & 124 & 68.2 & 78.9 \\
\hline & & 435016121284702 & 20030407 & 1202 & 143 & 60.7 & 87.1 \\
\hline & & Mean & - & - & - & - & - \\
\hline & & Standard deviation & - & - & - & - & - \\
\hline
\end{tabular}


[Date as year, month, day (YYYYMMDD); Time in hours and minutes, military; Organic wastewater compounds in micrograms per liter; surrogate recoveries in percent;

parameter codes for organic wastewater compound names: Pxxxx; "E", estimated; "<", less than; $\leq$, less than equal to; "M", presence verified, not quantified]

\begin{tabular}{|c|c|c|c|c|c|c|c|c|c|c|c|c|}
\hline $\begin{array}{l}\text { Type of onsite } \\
\text { wastewater } \\
\text { treatment system }\end{array}$ & Sample type or mathematical calculation & Station number & Date & Time & 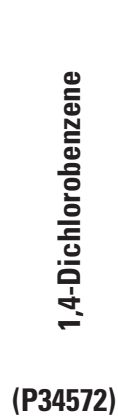 & 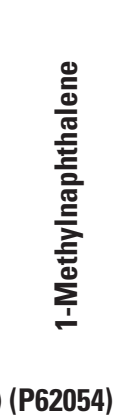 & 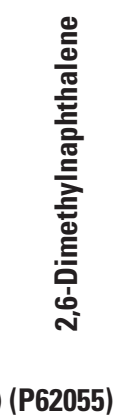 & (P62056) & 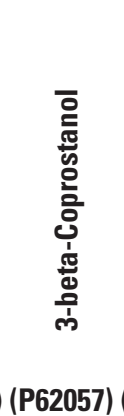 & 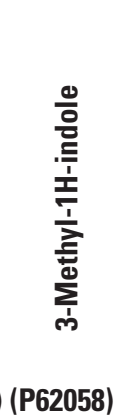 & 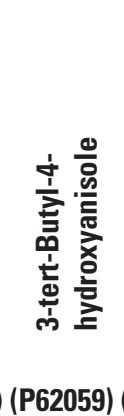 & 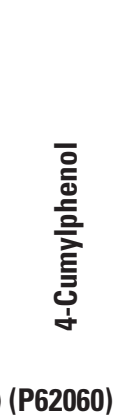 \\
\hline NAYADIC & $\begin{array}{l}\text { Septic tank effluent, environmental sample (analyte } \\
\text { concentrations in micrograms per liter, surrogate } \\
\text { recoveries in percent) }\end{array}$ & 435016121284702 & 20030407 & 1200 & $<5$ & E0.2 & $<5$ & E0.2 & E11 & 52 & $<5$ & $<5$ \\
\hline \multirow[t]{5}{*}{ NAYADIC } & $\begin{array}{l}\text { Septic tank effluent, spiked sample (analyte } \\
\text { concentrations in micrograms per liter, surrogate } \\
\text { recoveries in percent) }\end{array}$ & 435016121284702 & 20030407 & 1203 & E3.6 & 3.8 & 3.8 & 3.8 & E24 & 60 & E6 & 4 \\
\hline & $\begin{array}{l}\text { Mass of analyte added to the } 345 \text {-milliliter spike } \\
\text { sample (micrograms) }\end{array}$ & & & & 2.0 & 2.0 & 2.0 & 2.0 & 8.0 & 2.0 & 2.0 & 2.0 \\
\hline & $\begin{array}{l}\text { Expected concentration from spike (micrograms per } \\
\text { liter) }\end{array}$ & & & & 5.8 & 5.8 & 5.8 & 5.8 & 23.2 & 5.8 & 5.8 & 5.8 \\
\hline & $\begin{array}{l}\text { Recovered spike concentration (concentration in } \\
\text { spiked sample—concentration in environmental } \\
\text { sample) (micrograms per liter) }\end{array}$ & & & & $\leq 3.6$ & 3.6 & $\leq 3.8$ & 3.6 & 13 & 8 & $\leq 6$ & $\leq 4$ \\
\hline & Recovery (percent) & & & & $\leq 62$ & 62 & $\leq 66$ & 62 & 56 & 140 & $\leq 100$ & $\leq 69$ \\
\hline
\end{tabular}


Table B2. Results of laboratory organic wastewater compound matrix spike of septic tank effluent, La Pine, Oregon, 2003.—Continued

[Date as year, month, day (YYYYMMDD); Time in hours and minutes, military; Organic wastewater compounds in micrograms per liter; surrogate recoveries in percent;

parameter codes for organic wastewater compound names: Pxxxx; "E", estimated; "<", less than; $\leq$, less than equal to; "M", presence verified, not quantified]

\begin{tabular}{|c|c|c|c|c|c|c|c|c|c|c|c|c|}
\hline $\begin{array}{l}\text { Type of onsite } \\
\text { wastewater } \\
\text { treatment system }\end{array}$ & Sample type or mathematical calculation & Station number & Date & Time & 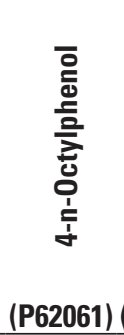 & 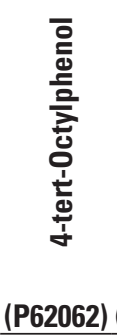 & 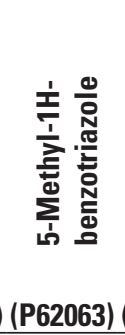 & 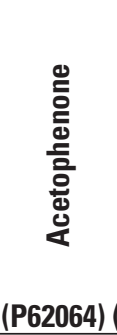 & 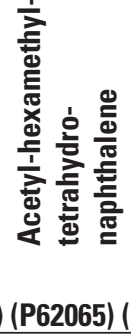 & 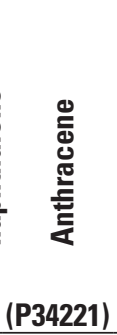 & 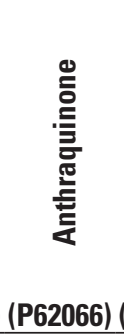 & 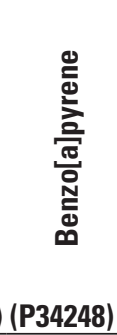 \\
\hline NAYADIC & $\begin{array}{l}\text { Septic tank effluent, environmental sample (analyte } \\
\text { concentrations in micrograms per liter, surrogate } \\
\text { recoveries in percent) }\end{array}$ & 435016121284702 & 20030407 & 1200 & $<5$ & $<5$ & $<5$ & $<5$ & E0.3 & $<5$ & $<5$ & $<5$ \\
\hline \multirow[t]{4}{*}{ NAYADIC } & $\begin{array}{l}\text { Septic tank effluent, spiked sample (analyte } \\
\text { concentrations in micrograms per liter, surrogate } \\
\text { recoveries in percent) }\end{array}$ & 435016121284702 & 20030407 & 1203 & 4 & 5 & E33 & 6.8 & 3.7 & 4.6 & 5 & 3.3 \\
\hline & $\begin{array}{l}\text { Mass of analyte added to the } 345 \text {-milliliter spike } \\
\text { sample (micrograms) }\end{array}$ & & & & 2.0 & 2.0 & 8.0 & 2.0 & 2.0 & 2.0 & 2.0 & 2.0 \\
\hline & $\begin{array}{l}\text { Expected concentration from spike (micrograms per } \\
\text { liter) }\end{array}$ & & & & 5.8 & 5.8 & 23.2 & 5.8 & 5.8 & 5.8 & 5.8 & 5.8 \\
\hline & $\begin{array}{l}\text { Recovered spike concentration (concentration in } \\
\text { spiked sample—concentration in environmental } \\
\text { sample) (micrograms per liter) }\end{array}$ & & & & $\leq 4$ & $\leq 5$ & $\leq 33$ & $\leq 6.8$ & 3.4 & $\leq 4.6$ & $\leq 5$ & $\leq 3.3$ \\
\hline
\end{tabular}




\begin{tabular}{|c|c|c|c|c|c|c|c|c|c|c|c|c|}
\hline $\begin{array}{l}\text { Type of onsite } \\
\text { wastewater } \\
\text { treatment system }\end{array}$ & Sample type or mathematical calculation & Station number & Date & Time & 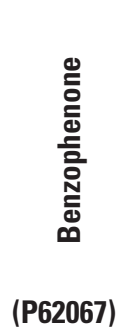 & 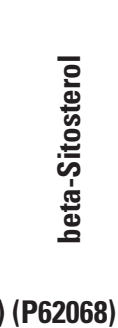 & 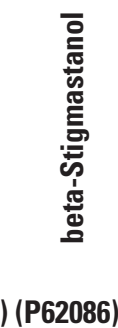 & (P62069) & 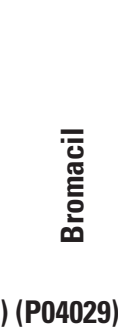 & 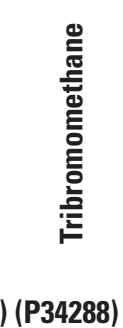 & 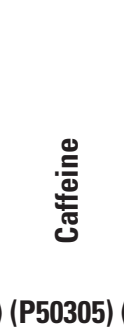 & (P62070) \\
\hline NAYADIC & $\begin{array}{l}\text { Septic tank effluent, environmental sample (analyte } \\
\text { concentrations in micrograms per liter, surrogate } \\
\text { recoveries in percent) }\end{array}$ & 435016121284702 & 20030407 & 1200 & 2.2 & E12 & E8 & M & $<5$ & $<5$ & 18 & 0.8 \\
\hline \multirow[t]{5}{*}{ NAYADIC } & $\begin{array}{l}\text { Septic tank effluent, spiked sample (analyte } \\
\text { concentrations in micrograms per liter, surrogate } \\
\text { recoveries in percent) }\end{array}$ & 435016121284702 & 20030407 & 1203 & 6.6 & E17 & E14 & E5 & 16 & $\mathrm{E} 4.5$ & 22 & 5.6 \\
\hline & $\begin{array}{l}\text { Mass of analyte added to the } 345 \text {-milliliter spike } \\
\text { sample (micrograms) }\end{array}$ & & & & 2.0 & 8.0 & 8.0 & 2.0 & 8.0 & 2.0 & 2.0 & 2.0 \\
\hline & $\begin{array}{l}\text { Expected concentration from spike (micrograms per } \\
\text { liter) }\end{array}$ & & & & 5.8 & 23.2 & 23.2 & 5.8 & 23.2 & 5.8 & 5.8 & 5.8 \\
\hline & $\begin{array}{l}\text { Recovered spike concentration (concentration in } \\
\text { spiked sample-concentration in environmental } \\
\text { sample) (micrograms per liter) }\end{array}$ & & & & 4.4 & 5 & 6 & $\leq 5$ & $\leq 16$ & $\leq 4.5$ & 4 & 4.8 \\
\hline & Recovery (percent) & & & & 76 & 22 & 26 & $\leq 86$ & $\leq 69$ & $\leq 78$ & 69 & 83 \\
\hline
\end{tabular}


Table B2. Results of laboratory organic wastewater compound matrix spike of septic tank effluent, La Pine, Oregon, 2003.—Continued

[Date as year, month, day (YYYYMMDD); Time in hours and minutes, military; Organic wastewater compounds in micrograms per liter; surrogate recoveries in percent;

parameter codes for organic wastewater compound names: Pxxxx; "E", estimated; "<", less than; $\leq$, less than equal to; "M", presence verified, not quantified]

\begin{tabular}{|c|c|c|c|c|c|c|c|c|c|c|c|c|}
\hline $\begin{array}{l}\text { Type of onsite } \\
\text { wastewater }\end{array}$ & & & & & 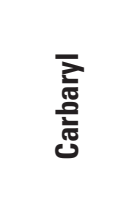 & 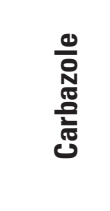 & 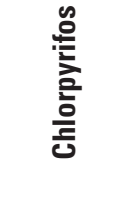 & 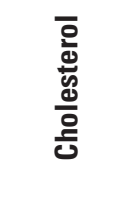 & 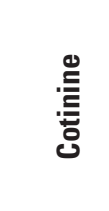 & 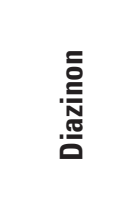 & $\begin{array}{l}\text { o. } \\
\frac{0}{0} \\
\frac{0}{\bar{c}} \\
\frac{0}{0}\end{array}$ & 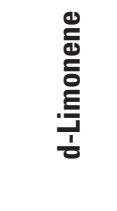 \\
\hline NAYADIC & $\begin{array}{l}\text { Septic tank effluent, environmental sample (analyte } \\
\text { concentrations in micrograms per liter, surrogate } \\
\text { recoveries in percent) }\end{array}$ & 435016121284702 & 20030407 & 1200 & $<5$ & $<5$ & $<5$ & E32 & $<5$ & $<5$ & $<5$ & E8.9 \\
\hline \multirow[t]{5}{*}{ NAYADIC } & $\begin{array}{l}\text { Septic tank effluent, spiked sample (analyte } \\
\text { concentrations in micrograms per liter, surrogate } \\
\text { recoveries in percent) }\end{array}$ & 435016121284702 & 20030407 & 1203 & E8 & 4.3 & 3.7 & $\mathrm{E} 40$ & 20 & 5 & E5 & E20 \\
\hline & $\begin{array}{l}\text { Mass of analyte added to the } 345 \text {-milliliter spike } \\
\text { sample (micrograms) }\end{array}$ & & & & 2.0 & 2.0 & 2.0 & 8.0 & 8.0 & 2.0 & 2.0 & 2.0 \\
\hline & $\begin{array}{l}\text { Expected concentration from spike (micrograms per } \\
\text { liter) }\end{array}$ & & & & 5.8 & 5.8 & 5.8 & 23.2 & 23.2 & 5.8 & 5.8 & 5.8 \\
\hline & $\begin{array}{l}\text { Recovered spike concentration (concentration in } \\
\text { spiked sample—concentration in environmental } \\
\text { sample) (micrograms per liter) }\end{array}$ & & & & $\leq 8$ & $\leq 4.3$ & $\leq 3.7$ & 8 & $\leq 20$ & $\leq 5$ & $\leq 5$ & 11 \\
\hline & Recovery (percent) & & & & $\leq 140$ & $\leq 74$ & $\leq 64$ & 35 & $\leq 86$ & $\leq 86$ & $\leq 86$ & 190 \\
\hline
\end{tabular}




\begin{tabular}{|c|c|c|c|c|c|c|c|c|c|c|c|c|}
\hline $\begin{array}{l}\text { Type of onsite } \\
\text { wastewater } \\
\text { treatment system }\end{array}$ & Sample type or mathematical calculation & Station number & Date & Time & 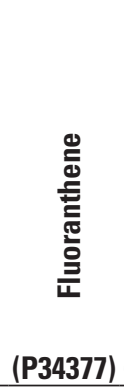 & 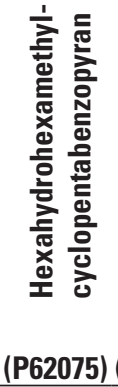 & $\begin{array}{c}\frac{0}{ㅇ ㅡ ㄹ ~} \\
\text { (P62076) }\end{array}$ & $\begin{array}{c}\overline{0} \\
\stackrel{\Xi}{ \pm} \\
\text { 응 } \\
\text { (P62077) }\end{array}$ & $\begin{array}{c}\text { 음 } \\
\text { 흫 } \\
\text { (P34409) } \\
\text { (Po }\end{array}$ & 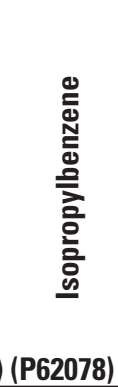 & $\begin{array}{c}\text { ․ㅡㅎ } \\
\text { 틀 } \\
\text { (P62079) }\end{array}$ & $\begin{array}{c}\overline{\overline{0}} \\
\stackrel{\bar{E}}{\bar{E}} \\
\text { (P62080) }\end{array}$ \\
\hline NAYADIC & $\begin{array}{l}\text { Septic tank effluent, environmental sample (analyte } \\
\text { concentrations in micrograms per liter, surrogate } \\
\text { recoveries in percent) }\end{array}$ & 435016121284702 & 20030407 & 1200 & $<5$ & 1.2 & 34 & $<5$ & $<5$ & $<5$ & $<5$ & 62 \\
\hline \multirow[t]{5}{*}{ NAYADIC } & $\begin{array}{l}\text { Septic tank effluent, spiked sample (analyte } \\
\text { concentrations in micrograms per liter, surrogate } \\
\text { recoveries in percent) }\end{array}$ & 435016121284702 & 20030407 & 1203 & 4.2 & 5.9 & 45 & 6.8 & 5.7 & E2.4 & 5.6 & 78 \\
\hline & $\begin{array}{l}\text { Mass of analyte added to the } 345 \text {-milliliter spike } \\
\text { sample (micrograms) }\end{array}$ & & & & 2.0 & 2.0 & 2.0 & 2.0 & 2.0 & 2.0 & 2.0 & 2.0 \\
\hline & $\begin{array}{l}\text { Expected concentration from spike (micrograms per } \\
\text { liter) }\end{array}$ & & & & 5.8 & 5.8 & 5.8 & 5.8 & 5.8 & 5.8 & 5.8 & 5.8 \\
\hline & $\begin{array}{l}\text { Recovered spike concentration (concentration in } \\
\text { spiked sample—concentration in environmental } \\
\text { sample) (micrograms per liter) }\end{array}$ & & & & $\leq 4.2$ & 4.7 & 11 & $\leq 6.8$ & $\leq 5.7$ & $\leq 2.4$ & $\leq 5.6$ & 16 \\
\hline & Recovery (percent) & & & & $\leq 72$ & 81 & 190 & $\leq 120$ & $\leq 98$ & $\leq 41$ & $\leq 97$ & 280 \\
\hline
\end{tabular}


Table B2. Results of laboratory organic wastewater compound matrix spike of septic tank effluent, La Pine, Oregon, 2003.—Continued

[Date as year, month, day (YYYYMMDD); Time in hours and minutes, military; Organic wastewater compounds in micrograms per liter; surrogate recoveries in percent;

parameter codes for organic wastewater compound names: Pxxxx; "E", estimated; "<", less than; $\leq$, less than equal to; "M", presence verified, not quantified]

\begin{tabular}{|c|c|c|c|c|c|c|c|c|c|c|c|c|}
\hline $\begin{array}{l}\text { Type of onsite } \\
\text { wastewater t } \\
\text { reatment system }\end{array}$ & Sample type or mathematical calculation & Station number & Date & Time & 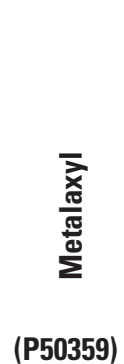 & 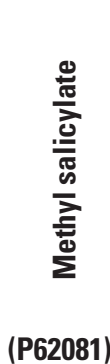 & 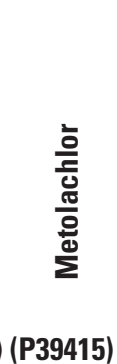 & 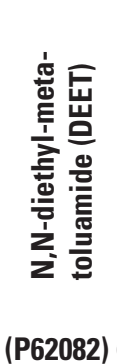 & 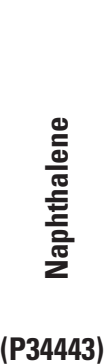 & 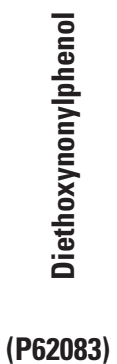 & 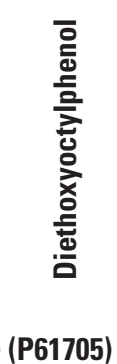 & 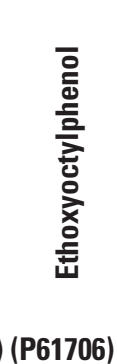 \\
\hline NAYADIC & $\begin{array}{l}\text { Septic tank effluent, environmental sample (analyte } \\
\text { concentrations in micrograms per liter, surrogate } \\
\text { recoveries in percent) }\end{array}$ & 435016121284702 & 20030407 & 1200 & $<5$ & $<5$ & $<5$ & 0.6 & E0.2 & E9 & $<5$ & $<5$ \\
\hline \multirow[t]{5}{*}{ NAYADIC } & $\begin{array}{l}\text { Septic tank effluent, spiked sample (analyte } \\
\text { concentrations in micrograms per liter, surrogate } \\
\text { recoveries in percent) }\end{array}$ & 435016121284702 & 20030407 & 1203 & 4.4 & 5.7 & 4 & 5.9 & 4.9 & E83 & E3 & E31 \\
\hline & $\begin{array}{l}\text { Mass of analyte added to the } 345 \text {-milliliter spike } \\
\text { sample (micrograms) }\end{array}$ & & & & 2.0 & 2.0 & 2.0 & 2.0 & 2.0 & 32 & 1.4 & 14 \\
\hline & $\begin{array}{l}\text { Expected concentration from spike (micrograms per } \\
\text { liter) }\end{array}$ & & & & 5.8 & 5.8 & 5.8 & 5.8 & 5.8 & 92.8 & 4.1 & 40.6 \\
\hline & $\begin{array}{l}\text { Recovered spike concentration (concentration in } \\
\text { spiked sample—concentration in environmental } \\
\text { sample) (micrograms per liter) }\end{array}$ & & & & $\leq 4.4$ & $\leq 5.7$ & $\leq 4$ & 5.3 & 4.7 & 74 & $\leq 3$ & $\leq 31$ \\
\hline & Recovery (percent) & & & & $\leq 76$ & $\leq 98$ & $\leq 69$ & 91 & 81 & 80 & $\leq 74$ & $\leq 76$ \\
\hline
\end{tabular}




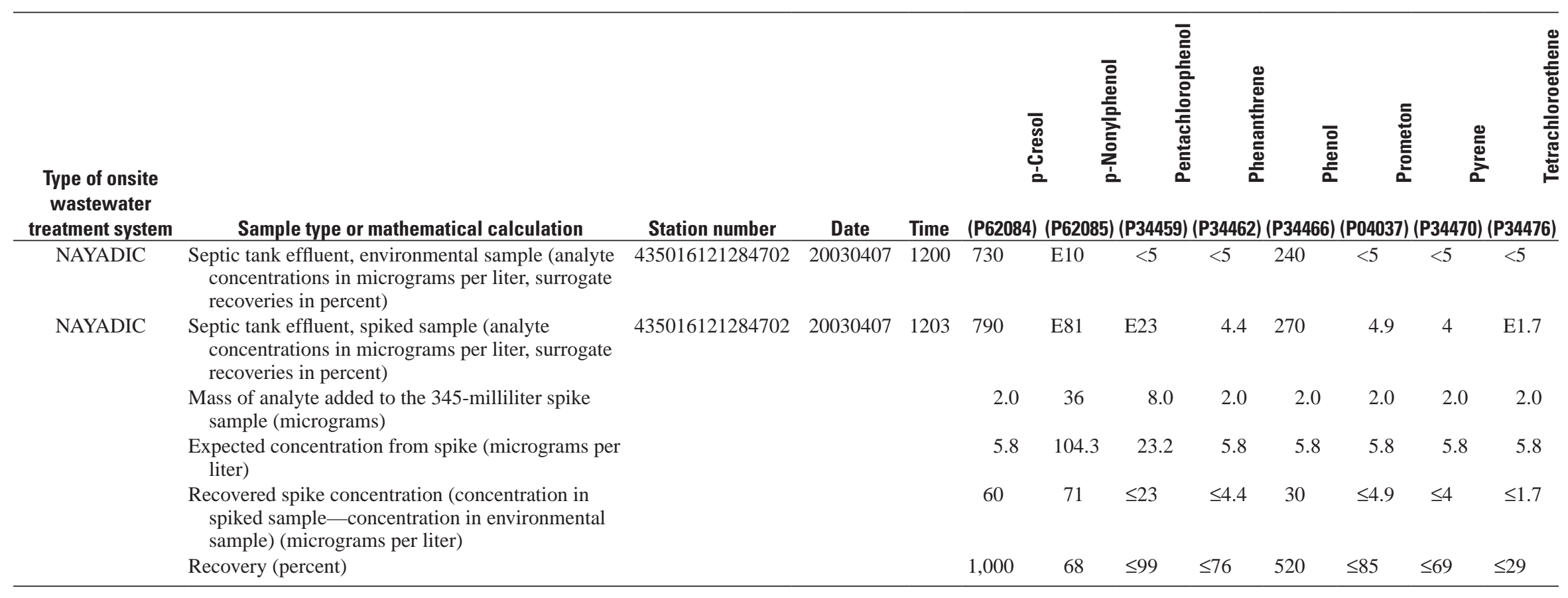


Table B2. Results of laboratory organic wastewater compound matrix spike of septic tank effluent, La Pine, Oregon, 2003.—Continued

[Date as year, month, day (YYYYMMDD); Time in hours and minutes, military; Organic wastewater compounds in micrograms per liter; surrogate recoveries in percent;

parameter codes for organic wastewater compound names: Pxxxx; "E", estimated; "<", less than; $\leq$, less than equal to; "M", presence verified, not quantified]

\begin{tabular}{|c|c|c|c|c|c|c|c|c|c|c|c|c|}
\hline \multirow{3}{*}{$\begin{array}{c}\begin{array}{c}\text { Type of onsite } \\
\text { wastewater } \\
\text { treatment system }\end{array} \\
\text { NAYADIC }\end{array}$} & \multirow[b]{2}{*}{ Sample type or mathematical calculation } & \multirow[b]{2}{*}{ Station number } & \multirow[b]{2}{*}{ Date } & \multirow[b]{2}{*}{ Time } & \multirow[t]{2}{*}{ 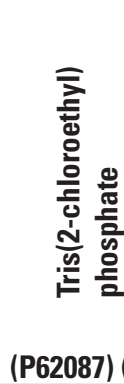 } & 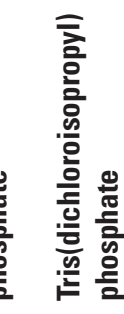 & 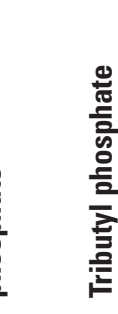 & \multirow{2}{*}{ 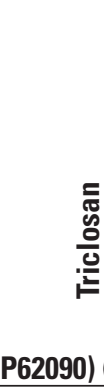 } & \multirow{2}{*}{ 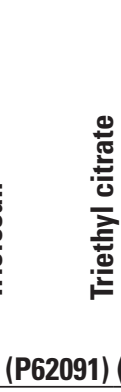 } & \multirow[t]{2}{*}{ (P62092) } & \multirow[t]{2}{*}{ 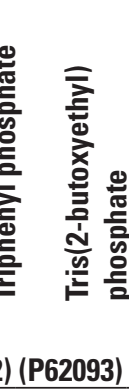 } & \multirow{2}{*}{ 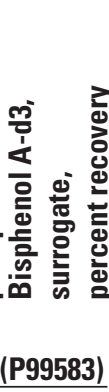 } \\
\hline & & & & & & (P62088) & ) (P62089) & & & & & \\
\hline & $\begin{array}{l}\text { Septic tank effluent, environmental sample (analyte } \\
\text { concentrations in micrograms per liter, surrogate } \\
\text { recoveries in percent) }\end{array}$ & 435016121284702 & 20030407 & 1200 & E0.4 & E0.4 & $<5$ & E2 & 0.6 & 0.8 & E2.2 & 78.6 \\
\hline \multirow[t]{5}{*}{ NAYADIC } & $\begin{array}{l}\text { Septic tank effluent, spiked sample (analyte } \\
\text { concentrations in micrograms per liter, surrogate } \\
\text { recoveries in percent) }\end{array}$ & 435016121284702 & 20030407 & 1203 & 5 & 3.9 & E4.7 & E7 & 5.4 & 5.2 & E7.2 & 77.7 \\
\hline & $\begin{array}{l}\text { Mass of analyte added to the } 345 \text {-milliliter spike } \\
\text { sample (micrograms) }\end{array}$ & & & & 2.0 & 2.0 & 2.0 & 2.0 & 2.0 & 2.0 & 2.0 & - \\
\hline & $\begin{array}{l}\text { Expected concentration from spike (micrograms per } \\
\text { liter) }\end{array}$ & & & & 5.8 & 5.8 & 5.8 & 5.8 & 5.8 & 5.8 & 5.8 & - \\
\hline & $\begin{array}{l}\text { Recovered spike concentration (concentration in } \\
\text { spiked sample—concentration in environmental } \\
\text { sample) (micrograms per liter) }\end{array}$ & & & & 4.6 & 3.5 & $\leq 4.7$ & 5 & 4.8 & 4.4 & 5.0 & - \\
\hline & Recovery (percent) & & & & 79 & 60 & $\leq 81$ & 86 & 83 & 76 & 86 & - \\
\hline
\end{tabular}


[Date as year, month, day (YYYYMMDD); Time in hours and minutes, military; Organic wastewater compounds in micrograms per liter; surrogate recoveries in percent; parameter codes for organic wastewater compound names: Pxxxx; "E”, estimated; "<", less than; $\leq$, less than equal to; "M", presence verified, not quantified]

\begin{tabular}{|c|c|c|c|c|c|c|c|}
\hline $\begin{array}{l}\text { Type of onsite } \\
\text { wastewater } \\
\text { treatment system }\end{array}$ & Sample type or mathematical calculation & Station number & Date & Time & 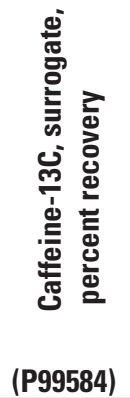 & 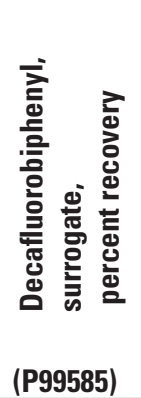 & 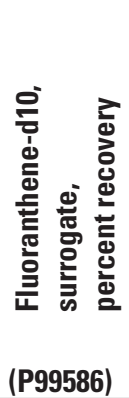 \\
\hline NAYADIC & $\begin{array}{l}\text { Septic tank effluent, environmental sample (analyte } \\
\text { concentrations in micrograms per liter, surrogate } \\
\text { recoveries in percent) }\end{array}$ & 435016121284702 & 20030407 & 1200 & 133 & 59.3 & 81.1 \\
\hline \multirow[t]{5}{*}{ NAYADIC } & $\begin{array}{l}\text { Septic tank effluent, spiked sample (analyte } \\
\text { concentrations in micrograms per liter, surrogate } \\
\text { recoveries in percent) }\end{array}$ & 435016121284702 & 20030407 & 1203 & 135 & 64.1 & 84.5 \\
\hline & $\begin{array}{l}\text { Mass of analyte added to the } 345 \text {-milliliter spike } \\
\text { sample (micrograms) }\end{array}$ & & & & & & \\
\hline & $\begin{array}{l}\text { Expected concentration from spike (micrograms per } \\
\text { liter) }\end{array}$ & & & & & & \\
\hline & $\begin{array}{l}\text { Recovered spike concentration (concentration in } \\
\text { spiked sample—concentration in environmental } \\
\text { sample) (micrograms per liter) }\end{array}$ & & & & & & \\
\hline & Recovery (percent) & & & & & & \\
\hline
\end{tabular}


Table B3. Summary statistics describing surrogate recovery data for all 77 environmental samples analyzed for organic wastewater compounds, La Pine, Oregon, 2003.

[Parameter code for constituents below constituent name; NWQL, National Water-Quality Laboratory; Mean recovery for all NWQL samples, listed for comparative purposes, describes mean surrogate recovery from analysis of 1,448 samples between October 1, 2002, and September 30, 2003, and was provided by S.D. Zaugg, U.S. Geological Survey, written commun., February 6, 2004]

Bisphenol A-d3, Caffeine-13C, Decafluorobiphenyl, Fluoranthene-d10,

surrogate surrogate surrogate surrogate

\section{Statistic} (P99583) surrogate
(P99586)

Minimum recovery, La Pine environmental samples (percent)

0

Maximum recovery, La Pine environmental samples (percent)

141

Median recovery, La Pine environmental samples (percent)

80.0

54.5

72.7

Mean recovery, La Pine environmental samples (percent)

$73.9-95.3$

113

124

Mean recovery, all NWQL samples (percent)

95.3

73.9

91.7 
[Date as year, month, day (YYYYMMDD); Time in hours and minutes, military; Organic wastewater compounds in micrograms per liter; surrogate recoveries in percent; parameter codes for organic wastewater compound names: Pxxxx; "E", estimated; "<", less than; "M", presence verified, not quantified]

\begin{tabular}{|c|c|c|c|c|c|c|c|c|c|c|c|c|c|c|c|}
\hline \multirow{2}{*}{$\begin{array}{l}\text { Type of onsite } \\
\text { wastewater } \\
\text { treatment system }\end{array}$} & \multirow{2}{*}{$\begin{array}{c}\text { Location in system where } \\
\text { field equipment blank } \\
\text { collected }\end{array}$} & \multirow{3}{*}{ Station number } & \multirow[b]{2}{*}{ Date } & \multirow{2}{*}{\multicolumn{2}{|c|}{ Time (P34572) }} & \multirow[t]{2}{*}{ 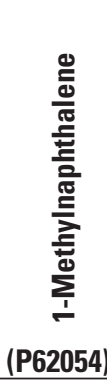 } & \multirow[t]{2}{*}{ 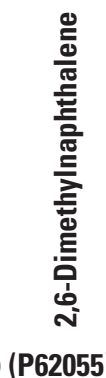 } & \multirow[t]{2}{*}{ 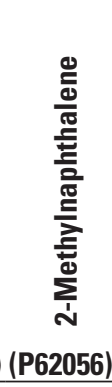 } & \multirow[t]{2}{*}{ 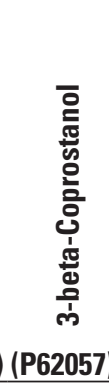 } & \multirow[t]{2}{*}{ 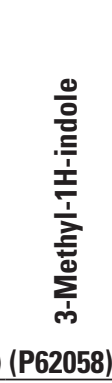 } & \multirow[t]{2}{*}{ 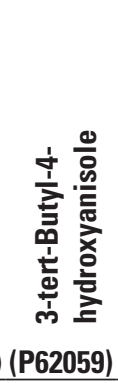 } & \multirow[t]{2}{*}{ 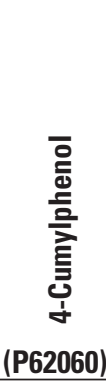 } & \multirow[t]{2}{*}{ 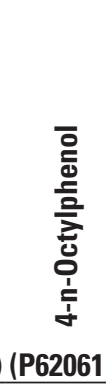 } & \multirow[t]{2}{*}{ 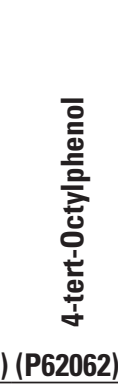 } & \multirow[t]{2}{*}{ 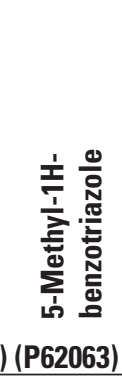 } \\
\hline & & & & & & & & & & & & & & & \\
\hline Pressure (standard) & Drainfield monitoring well & & 20030407 & 908 & $<0.5$ & $<0.5$ & $<0.5$ & $<0.5$ & $<2$ & $<1$ & $<5$ & $<1$ & $<1$ & $<1$ & $<2$ \\
\hline Puraflo & Septic tank effluent & 434010121325602 & 20030519 & 908 & M & $<0.5$ & $<0.5$ & $<0.5$ & $<2$ & $<1$ & $<5$ & $<1$ & $<1$ & $<1$ & $<2$ \\
\hline
\end{tabular}

\begin{tabular}{|c|c|c|c|c|c|c|c|c|c|c|c|c|c|c|c|}
\hline $\begin{array}{l}\text { Type of onsite } \\
\text { wastewater }\end{array}$ & $\begin{array}{l}\text { Location in system where } \\
\text { field equipment blank }\end{array}$ & & & & 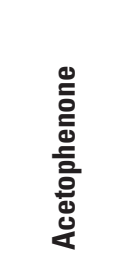 & 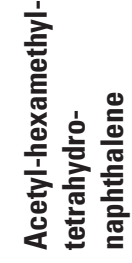 & 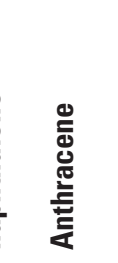 & 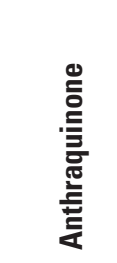 & 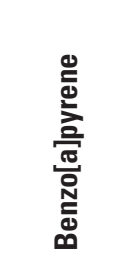 & 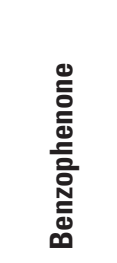 & 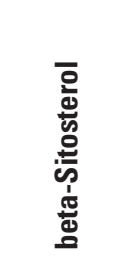 & 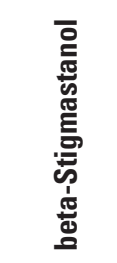 & 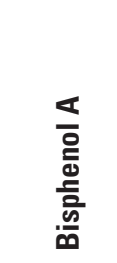 & 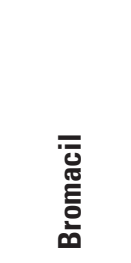 & 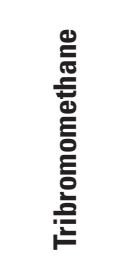 \\
\hline treatment system & collected & Station number & Date & ime & (P62064) & ) (P62065) & (P34221) & ) (P62066) & (P34248) & (P62067) & (P62068) & (P62086) & (P62069) & ) (P04029) ( & (P34288) \\
\hline Pressure (standard) & Drainfield monitoring well & 434248121295901 & 20030407 & 908 & $<0.5$ & $<0.5$ & $<0.5$ & $<0.5$ & $<0.5$ & $<0.5$ & $<2$ & $<2$ & $<1$ & $<0.5$ & $<0.5$ \\
\hline
\end{tabular}


Table B4. Results of analyses for organic wastewater compound field equipment blank samples, La Pine, Oregon, 2003.—Continued

[Date as year, month, day (YYYYMMDD); Time in hours and minutes, military; Organic wastewater compounds in micrograms per liter; surrogate recoveries in percent; parameter codes for organic wastewater compound names: Pxxxx; "E", estimated; “<", less than; "M", presence verified, not quantified]

\begin{tabular}{|c|c|c|c|c|c|c|c|c|c|c|c|c|c|c|c|}
\hline Pressure (standard) & Drainfield monitoring well & 434248121295901 & 20030407 & 908 & $<0.5$ & $<0.5$ & $<1$ & $<0.5$ & $<0.5$ & $<2$ & $<1$ & $<0.5$ & $<1$ & $<0.5$ & $<0.5$ \\
\hline Puraflo & Septic tank effluent & 434010121325602 & 20030519 & 908 & $<0.5$ & $<0.5$ & $<1$ & $<0.5$ & $<0.5$ & $<2$ & $<1$ & $<0.5$ & $<1$ & $<0.5$ & $<0.5$ \\
\hline
\end{tabular}

\begin{tabular}{|c|c|c|c|c|c|c|c|c|c|c|c|c|c|c|c|}
\hline $\begin{array}{l}\text { Type of onsite } \\
\text { wastewater }\end{array}$ & $\begin{array}{l}\text { Location in system where } \\
\text { field equipment blank }\end{array}$ & & & & 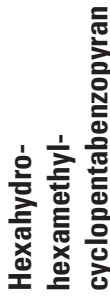 & $\begin{array}{l}\frac{0}{0} \\
\text { 을 }\end{array}$ & 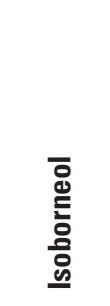 & 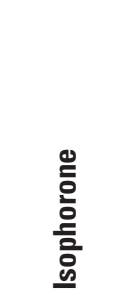 & 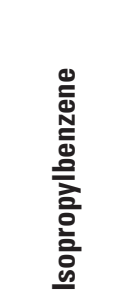 & 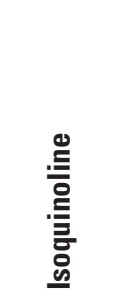 & 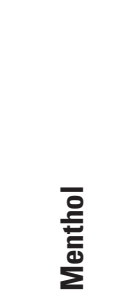 & 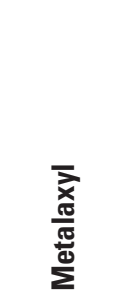 & 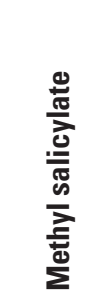 & 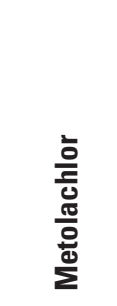 & 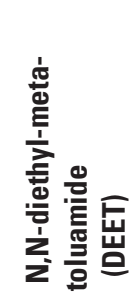 \\
\hline treatment system & collected & Station number & Date & Time & (P62075) & (P62076) & (P62077) & (P34409) & (P62078) & (P62079) & )(P62080) ( & (P50359) & (P62081) & (P39415) & 5) (P62082) \\
\hline Pressure (standard) & Drainfield monitoring well & 434248121295901 & 20030407 & 908 & $<0.5$ & $<0.5$ & $<0.5$ & $<0.5$ & $<0.5$ & $<0.5$ & $<0.5$ & $<0.5$ & $<0.5$ & $<0.5$ & $<0.5$ \\
\hline
\end{tabular}




\begin{tabular}{|c|c|c|c|c|c|c|c|c|c|c|c|c|c|c|c|c|}
\hline \multirow{2}{*}{$\begin{array}{l}\text { Type of onsite } \\
\text { wastewater } \\
\text { treatment system }\end{array}$} & \multirow{2}{*}{$\begin{array}{c}\text { Location in system where } \\
\text { field equipment blank } \\
\text { collected }\end{array}$} & \multirow[b]{2}{*}{ Station number } & \multirow[b]{2}{*}{ Date } & \multirow{2}{*}{\multicolumn{2}{|c|}{ Time (P34443) }} & \multirow[t]{2}{*}{ 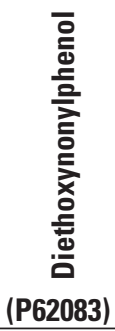 } & \multirow[t]{2}{*}{ 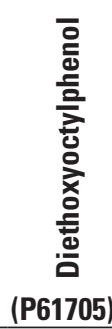 } & \multirow[t]{2}{*}{ 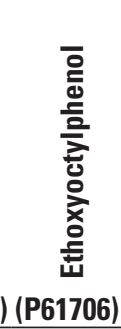 } & \multirow{2}{*}{ 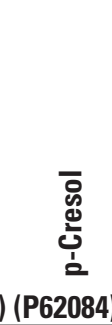 } & \multirow[t]{2}{*}{$\begin{array}{c}\overline{0} \\
\text { 흘 } \\
\text { 흘 } \\
\text { 흘 } \\
\text { P62085) }\end{array}$} & \multirow[t]{2}{*}{ 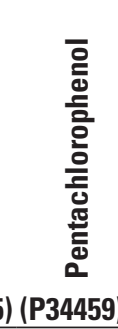 } & \multirow[t]{2}{*}{ 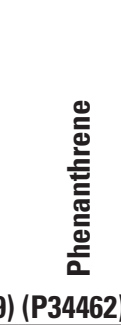 } & \multirow{2}{*}{$\begin{array}{l}\overline{ } \\
\stackrel{\bar{\Xi}}{\frac{E}{2}} \\
34466)\end{array}$} & \multirow[t]{2}{*}{ 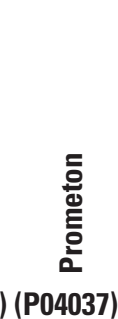 } & \multirow{2}{*}{ 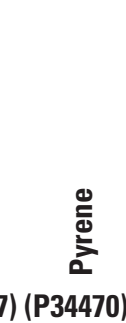 } & \\
\hline & & & & & & & & & & & & & & & & \\
\hline Pressure (standard) & Drainfield monitoring well & 434248121295901 & 20030407 & 908 & $<0.5$ & $<5$ & $<1$ & $<1$ & M & $<6$ & $<2$ & $<0.5$ & E0.4 & $<0.5$ & $<0.5$ & \\
\hline Puraflo & Septic tank effluent & 434010121325602 & 20030519 & 908 & $<0.5$ & $<5$ & $<1$ & $<1$ & $<1$ & $<6$ & $<2$ & $<0.5$ & $<0.5$ & $<0.5$ & $<0.5$ & \\
\hline $\begin{array}{l}\text { Type of onsite } \\
\text { wastewater }\end{array}$ & $\begin{array}{l}\text { Location in system where } \\
\text { field equipment blank }\end{array}$ & & & & 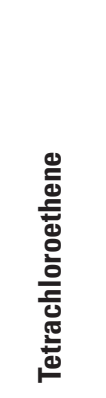 & 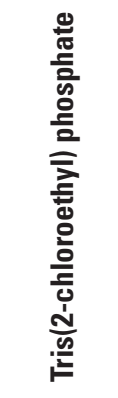 & 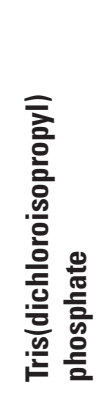 & 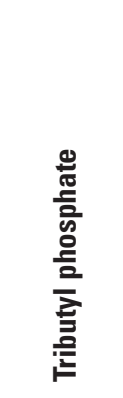 & $\begin{array}{l}\text { 통 } \\
\text { 을 } \\
\text { :른 }\end{array}$ & 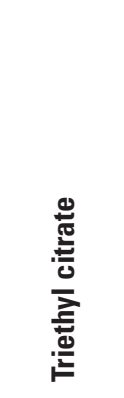 & 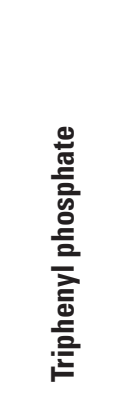 & 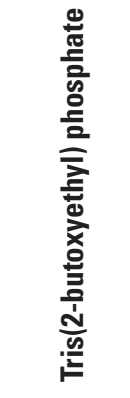 & 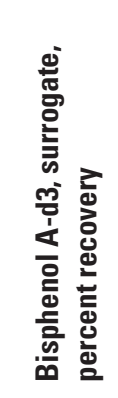 & 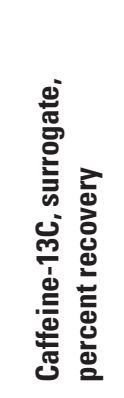 & 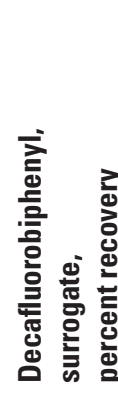 & 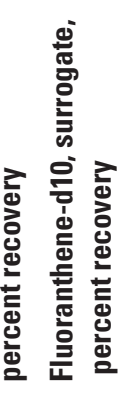 \\
\hline treatment system & collected & Station number & Date & Time (1 & (P34476) & (P62087) & (P62088) & (P62089) & (P62090) & $($ P62091) & (P62092) & (P62093) & (P99583) & (P99584) & (P99585) & ) (P99586 \\
\hline Pressure (standard) & Drainfield monitoring well & 434248121295901 & 20030407 & 908 & $<0.5$ & $<0.5$ & $<0.5$ & $<0.5$ & $<1$ & $<0.5$ & $<0.5$ & $<0.5$ & 15.7 & 97.1 & 75.6 & 91.0 \\
\hline Puraflo & Septic tank effluent & 434010121325602 & 20030519 & 908 & $<0.5$ & $<0.5$ & $<0.5$ & $<0.5$ & $<1$ & $<0.5$ & $<0.5$ & $<0.5$ & 47.1 & 88.2 & 82.4 & 88.2 \\
\hline
\end{tabular}




\section{Appendix C: Pharmaceutical Quality Assurance}

Quality-control data were collected to assess pharmaceutical sampling and analytical precision and bias. Table C1 shows these quality-control data along with environmental data from the body of this report; the environmental data are combined with the quality-control data in this table because we draw upon both in the analysis presented in this appendix.

\section{Pharmaceutical Replicates}

One set of triplicate samples from a transect site was collected and analyzed to measure analytical precision. Detections above provisional LRLs were not observed in the triplicate samples.

\section{Pharmaceutical Matrix Spike}

One transect sample was spiked at the NWQL with known concentrations of pharmaceuticals. The resultant information provides a measure of analytical bias, specifically, of analyte recovery.

Concentrations of pharmaceuticals in the environmental sample that was associated with the spiked sample were below provisional LRLs. Analyte recovery in the spiked sample was calculated with the assumption that the environmental concentration was zero, but the calculated recovery was censored with a " $\leq$ " to allow for the possibility that the analyte may have been present in the environmental sample at a concentration greater than $0 \mu \mathrm{g} / \mathrm{L}$ and less than the provisional LRL for that analyte. However, because pharmaceutical concentrations in the spiked sample generally were more than an order of magnitude greater than the provisional LRLs (table C1), any presence of pharmaceuticals in the environmental sample prior to spiking would generally have resulted in only minor reductions in the uncensored recoveries.

Assuming that pharmaceutical concentrations in the environmental sample were $0 \mu \mathrm{g} / \mathrm{L}$, recoveries ranged from 30 percent to 210 percent of expected concentration. Of the 18 compounds, 13 (72 percent) had recoveries between 60 and 140 percent. The median recovery was 81 percent, and the mean recovery 85 percent.

\section{Pharmaceutical Surrogate Recoveries}

One surrogate compound (Ethyl Nicotinate-d4) was added to pharmaceutical samples to evaluate method performance. Pharmaceutical concentrations are not adjusted on the basis of surrogate recoveries. The surrogate recovery (3 percent) for the sample from Senior 1 (Station Number 434212121294201) was unusually low. The archived sample extract was reanalyzed on December 5, 2003; upon reanalysis, the surrogate recovery was still low ( 2 percent) and the pharmaceuticals again were below provisional LRLs. The matrix for that sample was not unusual, and it is possible that an error during surrogate addition occurred. Surrogate recoveries for the remaining environmental samples ranged from 52 percent to 87 percent, with a median of 73 percent and a mean of 74 percent.

\section{Pharmaceutical Field Equipment Blanks}

Field equipment blanks were not collected. However, only two environmental samples contained pharmaceuticals at concentrations above provisional LRLs, indicating that sampling and analysis can generally be accomplished without introduction of significant contamination. 
Table C1. Environmental and quality-control data for pharmaceuticals from Senior transect, La Pine, Oregon, 2003.

[STE, septic tank effluent; Date as year, month, day (YYYYMMDD); Time in hours and minutes, military; Volume in milliliters; Chloride in milligrams per liter; Nitrite-plus-nitrate in milligrams $\mathrm{N}$ per liter; Pharmaceuticals in micrograms per liter, $\mu \mathrm{g} / \mathrm{L}$; "<", less than; "E", estimated (for cimetidine, estimated because recovery in laboratory spiked reagent grade water averages $<60$ percent; for other analytes, estimated because concentrations were greater than the highest calibration standard; matrix spike was 0.5 micrograms of each pharmaceutical compound in a 878.5-milliliter sample; archived extract of Senior 10 sample was reanalyzed December 5, 2003, confirming presence of acetaminophen ( 0.08 micrograms per liter) and caffeine ( 0.11 micrograms per liter); sulfamethoxazole was reported in the Senior 2 sample but not in the Senior STE sample; the sulfamethoxazole parent ion was present in the Senior STE sample at a concentration of 0.28 micrograms per liter but the confirmation ion was buried, and thus sulfamethoxazole was reported as a nondetect; --, no data; $\leq$, less than equal to]

\begin{tabular}{|c|c|c|c|c|c|}
\hline \multirow[b]{5}{*}{ Analyte } & \multirow{5}{*}{$\begin{array}{l}\text { Station number: } \\
\text { Station name: } \\
\text { Date: } \\
\text { Time: } \\
\text { Volume: }\end{array}$} & \multirow{5}{*}{$\begin{array}{c}434212121294299 \\
\text { Senior STE } \\
20030430 \\
1000 \\
304.2\end{array}$} & \multirow{5}{*}{$\begin{array}{c}434212121294201 \\
\text { Senior } 1 \\
20030429 \\
1100 \\
906.0\end{array}$} & \multirow{4}{*}{$\begin{array}{c}434212121294202 \\
\text { Senior } 2 \\
20030429 \\
1300\end{array}$} & \multirow{5}{*}{$\begin{array}{c}434212121294203 \\
\text { Senior } 3 \\
20030430 \\
1300 \\
954.8\end{array}$} \\
\hline & & & & & \\
\hline & & & & & \\
\hline & & & & & \\
\hline & & & & 954.6 & \\
\hline Chloride & & - & 5.22 & 6.26 & 14.5 \\
\hline Nitrite-plus-Nitrate & & - & 1.94 & 4.48 & 15.9 \\
\hline Cotinine & & 1.1 & $<0.01$ & $<0.01$ & $<0.01$ \\
\hline Salbutamol & & $<0.02$ & $<0.02$ & $<0.02$ & $<0.02$ \\
\hline Cimetidine & & E0.15 & $<0.01$ & $<0.01$ & $<0.01$ \\
\hline Acetaminophen & & E120 & $<0.04$ & $<0.04$ & $<0.04$ \\
\hline Ranitidine & & $<0.01$ & $<0.01$ & $<0.01$ & $<0.01$ \\
\hline 1,7-dimethylxanthine & & E58 & $<0.14$ & $<0.14$ & $<0.14$ \\
\hline Trimethoprim & & 0.19 & $<0.01$ & $<0.01$ & $<0.01$ \\
\hline Diltiazem & & $<0.02$ & $<0.02$ & $<0.02$ & $<0.02$ \\
\hline Warfarin & & $<0.01$ & $<0.01$ & $<0.01$ & $<0.01$ \\
\hline Ibuprofen & & $<0.04$ & $<0.04$ & $<0.04$ & $<0.04$ \\
\hline Gemfibrozil & & $<0.01$ & $<0.01$ & $<0.01$ & $<0.01$ \\
\hline Caffeine & & E110 & $<0.02$ & $<0.02$ & $<0.02$ \\
\hline Sulfamethoxazole & & $<0.06$ & $<0.06$ & 0.10 & $<0.06$ \\
\hline Dehydronifedipine & & $<0.02$ & $<0.02$ & $<0.02$ & $<0.02$ \\
\hline Codeine & & 0.066 & $<0.02$ & $<0.02$ & $<0.02$ \\
\hline Thiabendazole & & $<0.01$ & $<0.01$ & $<0.01$ & $<0.01$ \\
\hline Diphenhydramine & & 0.072 & $<0.01$ & $<0.01$ & $<0.01$ \\
\hline Carbamazapine & & $<0.01$ & $<0.01$ & $<0.01$ & $<0.01$ \\
\hline Ethyl Nicotinate-d4 Surrogate & & 2.87 & 0.028 & 0.78 & 0.54 \\
\hline Ethyl Nicotinate-d4 Surrogate & & 87 & 3 & 75 & 52 \\
\hline
\end{tabular}


Table C1. Environmental and quality-control data for pharmaceuticals from Senior transect, La Pine, Oregon, 2003._-Continued

[STE, septic tank effluent; Date as year, month, day (YYYYMMDD); Time in hours and minutes, military; Volume in milliliters; Chloride in milligrams per liter; Nitrite-plus-nitrate in milligrams $N$ per liter; Pharmaceuticals in micrograms per liter, $\mu \mathrm{g} / \mathrm{L}$; "<", less than; "E", estimated (for cimetidine, estimated because recovery in laboratory spiked reagent grade water averages $<60$ percent; for other analytes, estimated because concentrations were greater than the highest calibration standard; matrix spike was 0.5 micrograms of each pharmaceutical compound in a 878.5-milliliter sample; archived extract of Senior 10 sample was reanalyzed December 5, 2003, confirming presence of acetaminophen (0.08 micrograms per liter) and caffeine (0.11 micrograms per liter); sulfamethoxazole was reported in the Senior 2 sample but not in the Senior STE sample; the sulfamethoxazole parent ion was present in the Senior STE sample at a concentration of 0.28 micrograms per liter but the confirmation ion was buried, and thus sulfamethoxazole was reported as a nondetect; --, no data; $\leq$, less than equal to]

\begin{tabular}{|c|c|c|c|c|c|}
\hline \multirow[b]{5}{*}{ Analyte } & \multirow{5}{*}{$\begin{array}{l}\text { Station number: } \\
\text { Station name: } \\
\text { Date: } \\
\text { Time: } \\
\text { Volume: }\end{array}$} & \multirow{5}{*}{$\begin{array}{c}434212121294204 \\
\text { Senior } 4 \\
20030430 \\
1600 \\
927.3\end{array}$} & \multirow{5}{*}{$\begin{array}{c}434212121294205 \\
\text { Senior } 5 \\
20030430 \\
1800 \\
970.7\end{array}$} & \multirow{4}{*}{$\begin{array}{c}434212121294206 \\
\text { Senior } 6 \\
20030501 \\
1000\end{array}$} & \multirow{5}{*}{$\begin{array}{c}434212121294207 \\
\text { Senior } 7 \\
20030501 \\
1300 \\
909.3\end{array}$} \\
\hline & & & & & \\
\hline & & & & & \\
\hline & & & & & \\
\hline & & & & 958.7 & \\
\hline Chloride & & 5.71 & 16.6 & 6.40 & 1.79 \\
\hline Nitrite-plus-Nitrate & & 4.79 & 17.8 & 4.82 & 0.65 \\
\hline Cotinine & & $<0.01$ & $<0.01$ & $<0.01$ & $<0.01$ \\
\hline Salbutamol & & $<0.02$ & $<0.02$ & $<0.02$ & $<0.02$ \\
\hline Cimetidine & & $<0.01$ & $<0.01$ & $<0.01$ & $<0.01$ \\
\hline Acetaminophen & & $<0.04$ & $<0.04$ & $<0.04$ & $<0.04$ \\
\hline Ranitidine & & $<0.01$ & $<0.01$ & $<0.01$ & $<0.01$ \\
\hline 1,7-dimethylxanthine & & $<0.14$ & $<0.14$ & $<0.14$ & $<0.14$ \\
\hline Trimethoprim & & $<0.01$ & $<0.01$ & $<0.01$ & $<0.01$ \\
\hline Diltiazem & & $<0.02$ & $<0.02$ & $<0.02$ & $<0.02$ \\
\hline Warfarin & & $<0.01$ & $<0.01$ & $<0.01$ & $<0.01$ \\
\hline Ibuprofen & & $<0.04$ & $<0.04$ & $<0.04$ & $<0.04$ \\
\hline Gemfibrozil & & $<0.01$ & $<0.01$ & $<0.01$ & $<0.01$ \\
\hline Caffeine & & $<0.02$ & $<0.02$ & $<0.02$ & $<0.02$ \\
\hline Sulfamethoxazole & & $<0.06$ & $<0.06$ & $<0.06$ & $<0.06$ \\
\hline Dehydronifedipine & & $<0.02$ & $<0.02$ & $<0.02$ & $<0.02$ \\
\hline Codeine & & $<0.02$ & $<0.02$ & $<0.02$ & $<0.02$ \\
\hline Thiabendazole & & $<0.01$ & $<0.01$ & $<0.01$ & $<0.01$ \\
\hline Diphenhydramine & & $<0.01$ & $<0.01$ & $<0.01$ & $<0.01$ \\
\hline Carbamazapine & & $<0.01$ & $<0.01$ & $<0.01$ & $<0.01$ \\
\hline Ethyl Nicotinate-d4 Surrogate & & 0.86 & 0.73 & 0.76 & 0.72 \\
\hline Ethyl Nicotinate-d4 Surrogate & & 80 & 71 & 73 & 66 \\
\hline
\end{tabular}


[STE, septic tank effluent; Date as year, month, day (YYYYMMDD); Time in hours and minutes, military; Volume in milliliters; Chloride in milligrams per liter; Nitrite-plus-nitrate in milligrams $\mathrm{N}$ per liter; Pharmaceuticals in micrograms per liter, $\mu \mathrm{g} / \mathrm{L}$; " ">, less than; "E", estimated (for cimetidine, estimated because recovery in laboratory spiked reagent grade water averages $<60$ percent; for other analytes, estimated because concentrations were greater than the highest calibration standard; matrix spike was 0.5 micrograms of each pharmaceutical compound in a 878.5-milliliter sample; archived extract of Senior 10 sample was reanalyzed December 5, 2003, confirming presence of acetaminophen ( 0.08 micrograms per liter) and caffeine ( 0.11 micrograms per liter); sulfamethoxazole was reported in the Senior 2 sample but not in the Senior STE sample; the sulfamethoxazole parent ion was present in the Senior STE sample at a concentration of 0.28 micrograms per liter but the confirmation ion was buried, and thus sulfamethoxazole was reported as a nondetect; --, no data; $\leq$, less than equal to]

\begin{tabular}{|c|c|c|c|c|c|}
\hline \multirow[b]{5}{*}{ Analyte } & Station number: & 434212121294208 & 434212121294209 & 434212121294210 & 434212121294211 \\
\hline & Station name: & Senior 8 & Senior 9 & Senior 10 & Senior 11 \\
\hline & Date: & 20030501 & 20030501 & 20030502 & 20030502 \\
\hline & Time: & 1500 & 1700 & 1100 & 1200 \\
\hline & Volume: & 917.9 & 896.2 & 939.5 & 926.1 \\
\hline \multicolumn{2}{|l|}{ Chloride } & 12.6 & 1.09 & 3.30 & 1.78 \\
\hline \multicolumn{2}{|l|}{ Nitrite-plus-Nitrate } & 13.4 & 0.11 & 1.55 & 0.27 \\
\hline \multicolumn{2}{|l|}{ Cotinine } & $<0.01$ & $<0.01$ & $<0.01$ & $<0.01$ \\
\hline \multicolumn{2}{|l|}{ Salbutamol } & $<0.02$ & $<0.02$ & $<0.02$ & $<0.02$ \\
\hline \multicolumn{2}{|l|}{ Cimetidine } & $<0.01$ & $<0.01$ & $<0.01$ & $<0.01$ \\
\hline \multicolumn{2}{|l|}{ Acetaminophen } & $<0.04$ & $<0.04$ & 0.12 & $<0.04$ \\
\hline \multicolumn{2}{|l|}{ Ranitidine } & $<0.01$ & $<0.01$ & $<0.01$ & $<0.01$ \\
\hline \multicolumn{2}{|l|}{ 1,7-dimethylxanthine } & $<0.14$ & $<0.14$ & $<0.14$ & $<0.14$ \\
\hline \multicolumn{2}{|l|}{ Trimethoprim } & $<0.01$ & $<0.01$ & $<0.01$ & $<0.01$ \\
\hline \multicolumn{2}{|l|}{ Diltiazem } & $<0.02$ & $<0.02$ & $<0.02$ & $<0.02$ \\
\hline \multicolumn{2}{|l|}{ Warfarin } & $<0.01$ & $<0.01$ & $<0.01$ & $<0.01$ \\
\hline \multicolumn{2}{|l|}{ Ibuprofen } & $<0.04$ & $<0.04$ & $<0.04$ & $<0.04$ \\
\hline \multicolumn{2}{|l|}{ Gemfibrozil } & $<0.01$ & $<0.01$ & $<0.01$ & $<0.01$ \\
\hline \multicolumn{2}{|l|}{ Caffeine } & $<0.02$ & $<0.02$ & 0.18 & $<0.02$ \\
\hline \multicolumn{2}{|l|}{ Sulfamethoxazole } & $<0.06$ & $<0.06$ & $<0.06$ & $<0.06$ \\
\hline \multicolumn{2}{|l|}{ Dehydronifedipine } & $<0.02$ & $<0.02$ & $<0.02$ & $<0.02$ \\
\hline \multicolumn{2}{|l|}{ Codeine } & $<0.02$ & $<0.02$ & $<0.02$ & $<0.02$ \\
\hline \multicolumn{2}{|l|}{ Thiabendazole } & $<0.01$ & $<0.01$ & $<0.01$ & $<0.01$ \\
\hline \multicolumn{2}{|l|}{ Diphenhydramine } & $<0.01$ & $<0.01$ & $<0.01$ & $<0.01$ \\
\hline \multicolumn{2}{|l|}{ Carbamazapine } & $<0.01$ & $<0.01$ & $<0.01$ & $<0.01$ \\
\hline \multicolumn{2}{|c|}{ Ethyl Nicotinate-d4 Surrogate (micrograms per liter) } & 0.77 & 0.77 & 0.89 & 0.89 \\
\hline \multicolumn{2}{|c|}{ Ethyl Nicotinate-d4 Surrogate (recovery, percent) } & 71 & 69 & 84 & 83 \\
\hline
\end{tabular}


Table C1. Environmental and quality-control data for pharmaceuticals from Senior transect, La Pine, Oregon, 2003._-Continued

[STE, septic tank effluent; Date as year, month, day (YYYYMMDD); Time in hours and minutes, military; Volume in milliliters; Chloride in milligrams per liter; Nitrite-plus-nitrate in milligrams $\mathrm{N}$ per liter; Pharmaceuticals in micrograms per liter, $\mu \mathrm{g} / \mathrm{L}$; "<", less than; "E", estimated (for cimetidine, estimated because recovery in laboratory spiked reagent grade water averages $<60$ percent; for other analytes, estimated because concentrations were greater than the highest calibration standard; matrix spike was 0.5 micrograms of each pharmaceutical compound in a 878.5-milliliter sample; archived extract of Senior 10 sample was reanalyzed December 5, 2003, confirming presence of acetaminophen (0.08 micrograms per liter) and caffeine (0.11 micrograms per liter); sulfamethoxazole was reported in the Senior 2 sample but not in the Senior STE sample; the sulfamethoxazole parent ion was present in the Senior STE sample at a concentration of 0.28 micrograms per liter but the confirmation ion was buried, and thus sulfamethoxazole was reported as a nondetect; --, no data; $\leq$, less than equal to]

\begin{tabular}{|c|c|c|c|c|c|}
\hline \multirow{2}{*}{ Analyte } & $\begin{array}{l}\text { Station number: } \\
\text { Station name: } \\
\text { Date: } \\
\text { Time: } \\
\text { Volume: }\end{array}$ & $\begin{array}{c}434212121294208 \\
\text { Senior } 8 \text { Replicate } \\
20030501 \\
1501 \\
942.7 \\
\end{array}$ & $\begin{array}{c}434212121294208 \\
\text { Senior } 8 \text { Replicate } \\
20030501 \\
1502 \\
966.8 \\
\end{array}$ & \multicolumn{2}{|c|}{$\begin{array}{c}434212121294209 \\
\text { Senior } 9 \text { Matrix Spike } \\
20030501 \\
1703 \\
878.5 \\
\end{array}$} \\
\hline & & & & $\mu \mathbf{g} / \mathbf{L}$ & Recovery (percent) \\
\hline Chloride & & - & - & - & - \\
\hline Nitrite-plus-Nitrate & & - & - & - & - \\
\hline Cotinine & & $<0.01$ & $<0.01$ & 0.44 & $\leq 77$ \\
\hline Salbutamol & & $<0.02$ & $<0.02$ & 0.62 & $\leq 110$ \\
\hline Cimetidine & & $<0.01$ & $<0.01$ & 0.22 & $\leq 39$ \\
\hline Acetaminophen & & $<0.04$ & $<0.04$ & 1.2 & $\leq 210$ \\
\hline Ranitidine & & $<0.01$ & $<0.01$ & 0.17 & $\leq 30$ \\
\hline 1,7-dimethylxanthine & & $<0.14$ & $<0.14$ & 0.53 & $\leq 93$ \\
\hline Trimethoprim & & $<0.01$ & $<0.01$ & 0.44 & $\leq 76$ \\
\hline Diltiazem & & $<0.02$ & $<0.02$ & 0.23 & $\leq 40$ \\
\hline Warfarin & & $<0.01$ & $<0.01$ & 0.49 & $\leq 85$ \\
\hline Ibuprofen & & $<0.04$ & $<0.04$ & 0.41 & $\leq 71$ \\
\hline Gemfibrozil & & $<0.01$ & $<0.01$ & 0.52 & $\leq 92$ \\
\hline Caffeine & & $<0.02$ & $<0.02$ & 0.83 & $\leq 140$ \\
\hline Sulfamethoxazole & & $<0.06$ & $<0.06$ & 0.57 & $\leq 100$ \\
\hline Dehydronifedipine & & $<0.02$ & $<0.02$ & 0.52 & $\leq 91$ \\
\hline Codeine & & $<0.02$ & $<0.02$ & 0.42 & $\leq 74$ \\
\hline Thiabendazole & & $<0.01$ & $<0.01$ & 0.39 & $\leq 69$ \\
\hline Diphenhydramine & & $<0.01$ & $<0.01$ & 0.24 & $\leq 42$ \\
\hline Carbamazapine & & $<0.01$ & $<0.01$ & 0.49 & $\leq 85$ \\
\hline Ethyl Nicotinate-d4 Surrogate (micrograms per liter) & & 0.73 & 0.54 & 0.95 & - \\
\hline Ethyl Nicotinate-d4 Surrogate (recovery, percent) & & 68 & 52 & - & 84 \\
\hline
\end{tabular}


This page left intentionally blank 


\section{Appendix D: Coliphage Quality Assurance}

Quality-control data were collected to assess the precision and bias of coliphage sampling and analysis. These data are discussed in this appendix.

\section{Coliphage Replicates}

Nine sets of triplicate samples-four from septic tanks, four from lysimeters, and one from a transect well-were collected and analyzed to measure analytical precision (table D1). When coliphage were detected in septic tank effluent, the concentrations were highly variable, with the difference between the highest and lowest concentrations within one set of triplicates varying by more than two orders of magnitude (station number 434713121274302: 110, 14, and $<1 \mathrm{PFU} / 100 \mathrm{~mL}$ ). Of the lysimeters and ground-water samples, only one set contained detections (a lysimeter, station number 434207121324605: 140, 190, and $150 \mathrm{PFU} / 100$ $\mathrm{mL}$ ). With the large number of censored data (nondetects) in this data set, it cannot be determined whether water samples from lysimeters are inherently less variable in a relative sense than are samples of septic tank effluent. In an absolute sense, because coliphage concentrations are much higher in onsite wastewater than in ground water, sample-to-sample differences may be much greater in onsite wastewater than in ground water as well. Hydrodynamic dispersion would be expected to reduce concentration variability in ground water, too.

\section{Coliphage Matrix Spike}

One sample of septic tank effluent was spiked at the Ohio District Microbiology Laboratory to deliver coliphage at a target addition of $80 \mathrm{PFU} / 100 \mathrm{~mL}$ (table D2). The environmental sample yielded no detectable F-specific coliphage in the triplicate environmental samples, and $76 \mathrm{PFU} / 100 \mathrm{~mL}$ in the spiked sample, for a recovery of 95 percent (assum- ing $0 \mathrm{PFU} / 100 \mathrm{~mL}$ in the environmental sample). For somatic coliphage, the environmental samples contained 10,000, 6,100, and 12,000 PFU/100 mL in triplicate samples, and 15,000 PFU/100 mL in the spiked sample. The apparent surplus of 3,000 to 8,900 PFU/100 mL for an $80 \mathrm{PFU} / 100 \mathrm{~mL}$ spike likely represents primarily analytical and matrix variability. Spiking of septic tank effluent provides a useful measure of matrix effects when the environmental sample does not contain detectable coliphage. However, when the environmental sample contains high and variable concentrations of coliphage (as is the case here with the somatic coliphage), coliphage added in relatively small concentrations to the environmental sample cannot be discriminated from the coliphage already present.

\section{Coliphage Blanks}

Two field equipment blanks were collected by passing reagent-grade deionized water (the same organic-blank water used in the organic wastewater compound field equipment blanks) through sample collection equipment immediately prior to collecting environmental samples. In addition, one sample of routine-use deionized water was analyzed for coliphage; it was not a field equipment blank, but was analyzed to provide additional information about the microbial quality of project deionized water. No coliphage were detected in these samples (table D3). 
This page left intentionally blank 
Table D1. Results of replicate analyses for coliphage , La Pine, Oregon, 2003.

[USGS, U.S. Geological Survey; Sample type: STE, septic tank effluent, LYS, lysimeter; Date, as year, month, day; Time, military; mL, milliliters; <, less than; VQ, value qualifier code; d, diluted sample because method high range exceeded; —, no data]

\begin{tabular}{|c|c|c|c|c|c|c|}
\hline \multirow[b]{3}{*}{ Sample type } & \multirow[b]{3}{*}{ Station number } & \multirow[b]{3}{*}{$\begin{array}{c}\text { Sample } \\
\text { date/time }\end{array}$} & \multicolumn{4}{|c|}{ Method 1602-Single-agar layer } \\
\hline & & & \multicolumn{2}{|c|}{$\begin{array}{c}\text { F-Specific coliphage (USGS } \\
\text { parameter code 90904) }\end{array}$} & \multicolumn{2}{|c|}{$\begin{array}{c}\text { Somatic coliphage } \\
\text { (USGS parameter code 90903) }\end{array}$} \\
\hline & & & $\begin{array}{l}\text { Plaques per } \\
100 \mathrm{~mL}\end{array}$ & Vo & $\begin{array}{l}\text { Plaques per } \\
100 \mathrm{~mL}\end{array}$ & Vo \\
\hline STE & 435016121284702 & 200304071200 & $<1$ & - & 10,000 & $\mathrm{~d}$ \\
\hline STE & 435016121284702 & 200304071201 & $<1$ & - & 6,100 & $\mathrm{~d}$ \\
\hline STE & 435016121284702 & 200304071202 & $<1$ & - & 12,000 & $\mathrm{~d}$ \\
\hline STE & 434713121274302 & 200304231000 & $<1$ & - & 110 & - \\
\hline STE & 434713121274302 & 200304231001 & $<1$ & - & 14 & - \\
\hline STE & 434713121274302 & 200304231002 & $<1$ & - & $<1$ & - \\
\hline & & & & & & - \\
\hline LYS & 434247121305505 & 200305141100 & $<1$ & - & $<1$ & - \\
\hline LYS & 434247121305505 & 200305141101 & $<1$ & - & $<1$ & - \\
\hline LYS & 434247121305505 & 200305141102 & $<1$ & - & $<1$ & - \\
\hline STE & 434727121273702 & 200306041100 & $<1$ & - & 60 & - \\
\hline STE & 434727121273702 & 200306041101 & $<1$ & - & 150 & - \\
\hline STE & 434727121273702 & 200306041102 & $<1$ & - & 20 & - \\
\hline STE & 434908121291202 & 200311051200 & $<1$ & - & $<1$ & - \\
\hline STE & 434908121291202 & 200311051201 & $<1$ & - & 1 & - \\
\hline STE & 434908121291202 & 200311051202 & $<1$ & - & 1 & - \\
\hline LYS & 435016121284705 & 200311191200 & $<1$ & - & $<1$ & - \\
\hline LYS & 435016121284705 & 200311191201 & $<1$ & - & $<1$ & - \\
\hline LYS & 435016121284705 & 200311191202 & $<1$ & - & $<1$ & - \\
\hline
\end{tabular}


Table D1. Results of replicate analyses for coliphage , La Pine, Oregon, 2003.—Continued

[USGS, U.S. Geological Survey; Sample type: STE, septic tank effluent, LYS, lysimeter; Date as year, month, day (YYYYMMDD); Time, military; mL, milliliters; <, less than; VQ, value qualifier code; d, diluted sample because method high range exceeded; —, no data]

\begin{tabular}{|c|c|c|c|c|c|c|}
\hline \multirow[b]{3}{*}{ Sample type } & \multirow[b]{3}{*}{ Station number } & \multirow[b]{3}{*}{$\begin{array}{c}\text { Sample } \\
\text { date/time }\end{array}$} & \multicolumn{4}{|c|}{ Method 1602 - Single-agar layer } \\
\hline & & & \multicolumn{2}{|c|}{$\begin{array}{c}\text { F-Specific Coliphage (USGS } \\
\text { Parameter Code 90904) } \\
\end{array}$} & \multicolumn{2}{|c|}{$\begin{array}{c}\text { Somatic Coliphage } \\
\text { (USGS Parameter Code 90903) }\end{array}$} \\
\hline & & & $\begin{array}{l}\text { Plaques per } \\
100 \mathrm{~mL}\end{array}$ & vo & $\begin{array}{c}\text { Plaques per } \\
100 \mathrm{~mL}\end{array}$ & vo \\
\hline LYS & 434207121324605 & 200312080900 & $<1$ & - & 140 & - \\
\hline LYS & 434207121324605 & 200312080901 & $<1$ & - & 190 & - \\
\hline LYS & 434207121324605 & 200312080902 & $<1$ & - & 150 & - \\
\hline LYS & 434236121310505 & 200312080930 & $<1$ & - & $<1$ & - \\
\hline LYS & 434236121310505 & 200312080931 & $<1$ & - & $<1$ & - \\
\hline LYS & 434236121310505 & 200312080932 & $<1$ & - & $<1$ & - \\
\hline Transect well & 434212121294201 & 20034291100 & $<1$ & - & $<1$ & - \\
\hline Transect well & 434212121294201 & 20034291101 & $<1$ & - & $<1$ & - \\
\hline Transect well & 434212121294201 & 20034291102 & $<1$ & $=$ & $<1$ & - \\
\hline
\end{tabular}


Table D2. Results of laboratory coliphage matrix spike of septic tank effluent, La Pine, Oregon, 2003.

[USGS, U.S. Geological Survey; Sample type: STE, septic tank effluent; Date as year, month, day (YYYYMMDD); Time, military; mL, milliliters; <, less than; VQ, value qualifier code; d, diluted sample because method high range exceeded; matrix spike target addition: 80 plaque forming units per $100 \mathrm{~mL}$;

-, no data]

\begin{tabular}{|c|c|c|c|c|c|c|}
\hline \multirow[b]{3}{*}{ Sample type } & \multirow[b]{3}{*}{ Station number } & \multirow[b]{3}{*}{$\begin{array}{c}\text { Sample } \\
\text { date/time }\end{array}$} & \multicolumn{4}{|c|}{ Method 1602-Single-agar layer } \\
\hline & & & \multicolumn{2}{|c|}{$\begin{array}{c}\text { F-Specific coliphage } \\
\text { (USGS parameter code 90904) } \\
\end{array}$} & \multicolumn{2}{|c|}{$\begin{array}{c}\text { Somatic coliphage } \\
\text { (USGS parameter code } \\
\text { 90903) }\end{array}$} \\
\hline & & & $\begin{array}{c}\text { Plaques per } \\
100 \mathrm{~mL}\end{array}$ & Vo & $\begin{array}{c}\text { Plaques per } \\
100 \mathrm{~m})\end{array}$ & vo \\
\hline STE & 435016121284702 & 200304071200 & $<1$ & - & 10,000 & $\mathrm{~d}$ \\
\hline STE & 435016121284702 & 200304071201 & $<1$ & - & 6,100 & $\mathrm{~d}$ \\
\hline STE & 435016121284702 & 200304071202 & $<1$ & - & 12,000 & $\mathrm{~d}$ \\
\hline STE Matrix Spike & 435016121284702 & 200304071203 & 76 & - & 15,000 & $d$ \\
\hline
\end{tabular}


Table D3. Results of analysis of deionized water and of field equipment blanks for coliphage, La Pine, Oregon, 2003.

[USGS, U.S. Geological Survey; Sample type: DFMW, drainfield monitoring well, STE, septic tank effluent; Date, as year, month, day;

Time, military; $\mathrm{mL}$, milliliters; <, less than]

\begin{tabular}{|c|c|c|c|c|}
\hline \multirow[b]{3}{*}{ Sample type } & \multirow[b]{3}{*}{ Station number } & \multirow[b]{3}{*}{$\begin{array}{c}\text { Sample } \\
\text { date/time }\end{array}$} & \multicolumn{2}{|c|}{ Method 1602-Single-agar layer } \\
\hline & & & $\begin{array}{c}\text { F-Specific coliphage } \\
\text { (USGS parameter code } \\
\text { 90904) }\end{array}$ & $\begin{array}{c}\text { Somatic coliphage } \\
\text { (USGS parameter code } \\
\text { 90903) }\end{array}$ \\
\hline & & & Plaques per $100 \mathrm{~mL}$ & Plaques per $100 \mathrm{~mL}$ \\
\hline Deionized water & 453054122330601 & 200304071500 & $<1$ & $<1$ \\
\hline DFMW & 434248121295901 & 200304070908 & $<1$ & $<1$ \\
\hline STE & 434010121325602 & 200305190908 & $<1$ & $<1$ \\
\hline
\end{tabular}




\section{GLOSSARY OF SELECTED TERMS}

Advection The process by which solutes are transported by the bulk motion of flowing water.

Alluvium Clay, silt, sand, gravel, or other particulate rock material deposited by the actions of streams and rivers.

Generally unconsolidated or semiconsolidated.

Anthropogenic Resulting from or pertaining to human activities.

Aquifer A geologic formation, group of formations, or part of a formation containing sufficient water-saturated permeable material to yield or be capable of yielding usable quantities of water to wells and springs.

Bacterial lawn A continuous layer or cover of bacteria on the surface of a growth medium, used to culture coliphage for analysis.

Denitrification A process by which nitrate is reduced to nitrogen oxides or nitrogen gas.

Direct-push well A temporary or permanent monitoring well installed by applying force from a hydraulically powered percussion hammer and from the static weight of the directpush vehicle to a string of steel tools. The applied force advances the tools, displacing sediment in the process. Tools for a temporary monitoring well may consist of a disposable point, a sheathed well screen, and sections of (steel) casing. Tools for a permanent monitoring well are similar, but tend to be larger in diameter, and lack the sheathed well screen; polyvinyl chloride monitoring-well casing for permanent monitoring wells is installed inside of the steel casing, and then the steel casing is removed, leaving a permanent monitoring well behind.

Environmental sample A water sample collected from an aquifer or stream for the purpose of chemical, physical, or biological characterization of the sampled resource. (Compare with “quality-control sample.”)

Flowpath The pathway or course taken by particles of water and associated solutes as they move through an aquifer.

Laboratory reporting level (LRL) A censoring level for reporting laboratory analytical results. The LRL is established at a higher level (concentration) than the method detection limit (see "method detection limit") to provide a conservative approach to reporting analytical data. Concentrations between the method detection limit and the LRL may be reported for some analytical methods, such as the wastewater compound method used in this report; however, such data will be qualified with an "E” (for “estimated”) remark code.
Lysimeter A device used to collect a water sample from the unsaturated zone, somewhere between land surface and the water table.

Median The middle or central value in a distribution of data ranked in order of magnitude. The median is also known as the 50th percentile.

Method detection limit (MDL) The minimum concentration of a substance that can be measured and reported with 99-percent confidence that the analyte concentration is greater than zero (U.S. Environmental Protection Agency, 1997). The U.S. Geological Survey follows U.S. Environmental Protection Agency procedures for establishing method detection limits (U.S. Environmental Protection Agency, 1997). (Compare with “laboratory reporting level.”)

Potentiometric surface A map of values of static hydraulic head for an aquifer or a hydrologically related group of aquifers, contoured to facilitate interpretation of groundwater flowpaths. Equivalent to the water table elevation in an unconfined aquifer. (Static hydraulic head is the height above a standard datum of the surface of a column of water that can be supported by the static pressure at a given point in an aquifer.)

Project censoring level A project-specific censoring level that is greater than the laboratory reporting level (see "laboratory reporting level”). A project censoring level may be established if quality-control samples (see quality-control sample”) indicate the potential for sampling or analytical bias. In this report, project censoring levels were established for two wastewater compounds that were detected in blanks. The project censoring levels were set at 10 times observed contamination levels (U.S. Environmental Protection Agency, 1993).

Quality assurance Evaluation of quality-control data to allow quantitative determination of the quality of chemical data collected during the execution of a study. Techniques used to collect, process, and analyze water samples are evaluated.

Quality-control sample A water sample analyzed for quality-assurance purposes. (Compare with "environmental sample”; see “quality assurance.”)

Recharge The process of addition of water to an aquifer.

Redox Pertaining to or taking part in a coupled reduction and oxidation reaction.

Sorption Thermodynamically driven partitioning of a solute into a solid phase or onto a solid phase surface. 
This page left intentionally blank 


\section{Back cover:}

Top, the Deschutes River near South Century Drive. Much of the ground water in the La Pine area feeds into rivers such as the Deschutes. Ground water can be a source of contaminants to rivers.

Bottom, coliphage are identified by culturing them in a laboratory. (Coliphage are viruses that infect coliform bacteria, and are often found in high concentrations in human wastewater.) 


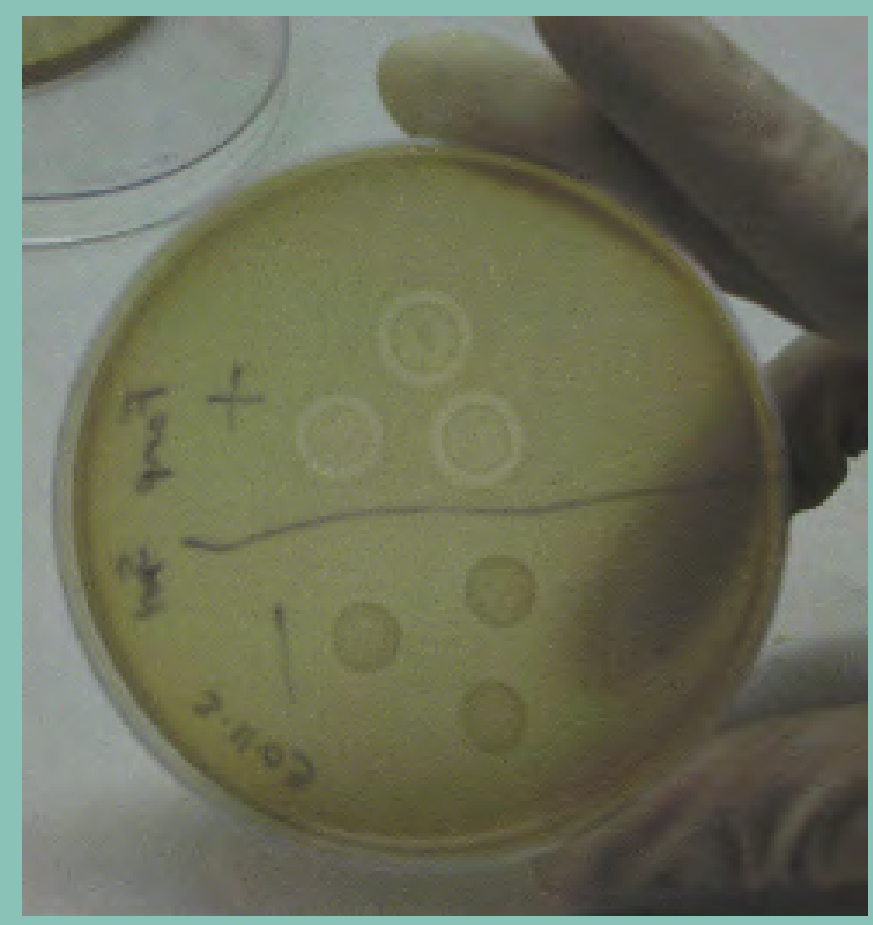

